DE83 017739

\title{
DISSOCIATIVE IONIZATION OF LIQUID WATER TNDUCED BY VIBRATIONAL OVERTONE EXCITATION
}

\author{
Wesley Charles Natzle \\ (Ph.D. Thesis)
Lawrence Berkeley Laboratory
Unversity of California
Bexkeley, California 94720
PORTIONS OF THIS SEPORT ARE ULEGIBLE.
Ii has heen reprodineed from the best aviliabie copy to parmit the broadest. possitie availability.

\section{DISCLAIMER}

\begin{abstract}
This report was prepared as an acoount of work sponeored by an agency of the United State Government. Neither the Uniled States Government nor any asency thereof, nor any of their employees, makes any wartanty, express or implied, or anumes any lezal linbility or retponesbility for the accurscy, completencess, or usefuleess of any information, apparatus, product, or process disclowed, of represents that its use would not infriage privately owned rights. Reletence herein to any spocific commercial product, procesu of servioe by trade nume, trademark, manufacturer, or otherwise doen not necemarily constitute or imply its endonement, recourmendation, or favoring by the Uniled Staten Government or any agency thereof. The viem and opinions of authors expresod berein do not mocesuarily stale or reflect thoee of the United State Government or any asency thereof.
\end{abstract}

This work was supported by the Director, Off \pm ce of Energy Research, Offtce of Basic Energy Sciences, Cheraical Sciences Division of the U.S. Department of Energy under Contract Number DE-ACO3-76SFo0098.<smiles>CCCCC1=CC=CC1C</smiles> 


\title{
Dissociative Ionization of Liquid water Induced by Vibrational Overtone Excitation
}

\author{
Wesley Charles Natzle
}

\section{ABSTRACT}

Photochemistry of vibrationally activated ground electronic state liquid water to produce $\mathrm{H}^{+}$and $\mathrm{OH}^{-}$ions has been initiated by pulsed, single-photon excitation of overtone and combination transitions. Transient conductivity measurements were used to determine quantum yields as a function of photon energy, isotopic composition, and temperature. The equilibrium relaxation rate following perturbation by the vibrationally actirated reaction was also measured as a function of temperature and isotopic composition.

In $\mathrm{H}_{2} \mathrm{O}$, the quantum yield at $283 \pm 1 \mathrm{~K}$ varies from $2 \times 10^{-9}$ to $4 \times 10^{-5}$ for wave numbers between 7605 and $18140 \mathrm{~cm}^{-1}$. A model is used to characterize the wavelength dependence. The model gives the quantum yield as the probability that photon absorption produces an ion pair times the probability of separation from the ion pair. The result is proportional to the ionic mobility, to the equilibrium constant of the ion pair and excited reactant and to the length of time the reactant remains activated. The quantum yield rises through the low frequency side of an absorption band and is relatively constant through 
the band center and high frequency wing. This behavior shows the quantum yield is nearly independent of the physical process that broadens the absorption. The process is either structural differences or combination excitation of lattice motions. The increase in quantum yield with temperature is smaller with higher excitation energy.

In $D_{2} O$, the dependence of quantum yield on wavelength has the same qualitative shape as for $\mathrm{H}_{2} \mathrm{O}$, but is shifted to lower quantum yields. The hydrogen mole fraction dependence is rot monotonic. The position of a minimum in the quantum yield versus hydrogen mole fraction curve is consistent with a lower quantum yield for excitation of HOD in $D_{2} O$ than for excitation of $D_{2} O$. This behavior can be explained by an unfavorable flow of energy from an excited $\mathrm{OH}$ stretch of an HOD when surrounded by $\mathrm{D}_{2} \mathrm{O}$.

The ionic recombination distance of $5.8 \pm 0.5$ is calculated from the equilibrium relaxation rate. It is constant within experimental error with temperature in $\mathrm{H}_{2} \mathrm{O}$ and with isotopic composition at $25 \pm 1^{\circ} \mathrm{C}$. The distance is consistent with recombination in a four-molecule hydrogen bonded linear chain.

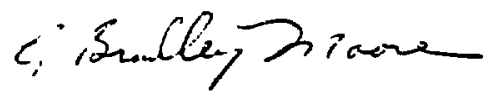


I dedicate this thesis to the nineteenth century philosopher/economist

John Stuart Mill

A man of genius, strength, and perseverance 


\section{ACKNOWLEDGEMENTS}

Before writing my acknowledgements, I decided to leaf through the acknowledgements written by some of my predecessors. The authors had two major goals: (1) to thank those who helped with their development as scientists; and (2) to thank people who offered non-scientific support. My goals include these two and add a third--to express a few light-hearted, philosophical thoughts on the writing of acknowledgements.

I can begin to reach the first goal by thanking Brad Moore. The multitude of successful scientists he has advised will testify to his effectiveness. The astute will learn from his example, especially his drive to find and clearly describe the heart of any matter. Several professors, in addition to Brad, were helpful in guiding me to the completion and understanding of my thesis. Professor David Goodall offered considerable help with the water experiments from far off England. Professor Gabor Somorjai helped me understand the purpose of this work. Finally, two professors gave me early guidance and continuing inspiration. Professor Grover Willis gave me a nudge toward chemistry and Professor John Root intensified my interest in kinetics.

My thanks are not complete without mention of Jim Weisshaar. We had a good lab on the "hill" when be was the "senior member" of the hill group. I greatly 
appreciated our major scientific discussions, but did not fully appreciate the countless small but significant things Jim did to make the hill a fun and efficient place to work. Other group members also need to be included. When I as a beginner was lost or confused, Don Douglas always took time to teach me a thing or two. Andy kung continues to put up with all of my "what if ..." and "why not ..." questions. The entire past and present Moore group deserves my thanks for enduring countless questions similar to those endured by Andy and for participating in the ensuing discussions.

Although many people have helped me increase my knowledge during graduate school, perhaps the strongest indication that I learned something from them is that I leave with more unanswered questions than when I arrived. I hope that the people I meet in the future will be as able to increase the number of my unanswered questions.

Several people deserve thanks for loans of equipment and supplies. Carol Balfe and the Raymond group provided feedstock water. Howard Gamper provided a Millipore filter holder and filters, and Bill vance provided septum tops.

The second part of most author's acknowledgements is included because they realize that often the support of greatest importance is non-scientific. My mother and father, Jane and Charles Natzle, my sister, Jeanette, and brother-in-law, Bill vance, have provided a needed "long 
term" family base. In addition, Paul whitmore, Mark Logan, and Erika Schneider as apartment mates and friends have been part of a Berkeley "family". Jackie Denney deserves special thanks for her efforts to extend the family to the research group as well. I have benefited from the friendship and conversation of Joan Frisoli, Alison Abbate, and Linda Young, enjoyed discussion with Chi-Ke Cheng, Hai-Lung Dai, Michael Diegelmann, and Tomoji Rawai. A fresh view of the United States and its relationship to the rest of the world never fails to stimulate thought. A list of people to thank would not be complete without inclusion of some people whose influence is sometimes subtle, often powerful: L. Vasudeva, Le-Ping Cheng, John Stuart Mill, Michael Novak, Karl Jaspers, Rierkegaard, the German Existentialists, J.S., Nietsche, Ayn Rand, Lao-Tzu, Aristotle, and the Berkeley Concert Band gang, especially Reiko Kubota for exercising her eyes and fingers by typing my thesis.

I have tried to follow the example of successful previous graduate students when writing my acknowledgements. Hopefully, I have not left out many people I would like to thank. I am ending my acknowledgements but not my acknowledgement section. I am dissatisfied with what I have just written for two reasons. First, my acknowledgements are not original. After scores of people have worked in the same research group, the emphasis of each acknowledgement is different, but the names are the same. Secondly, I find it 
difficult to write acknowledgements that accurately express my gratitude. I decided I could remedy the first difficulty by philosophizing about the second. None of my predecessors have considered the problem of writing good acknowledgements. I consider this my only opportunity to write something completely creative and unfettered by the laws of nature or probability.

Some may think this section inappropriate. It is true that my time would have been better spent writing something else, but I think the section is appropriate. Since it is about acknowledgements, I can include it with no disruption of the generally accepted thesis format. No additional sections need be added. Also, since one major goal of the thesis is to help the reader understand what the author has learned, why not include what I have learned about acknowledgements?

Other readers might think the section appropriate, but uninteresting. I risk losing the attention and respect of those not inclined toward philosophy. Acknowledgements almost write themselves. I began to understand acknowledgements only after they were written. Upon re-reading them, I realized they did not accurately express my feelings. The crux of the problem is that although feelings of gratitude persist, the ideal emphasis is ephemeral. No difficulty is caused by this until it is time to scrutinize and formulate these feelings into a snapshot called the acknowledgement 
section of a thesis. Then, almost as soon as they are written, the ideal emphasis changes. I did not immediately realize how the difficulty could be resolved. I only knew the acknowledgements I had did not match my vision of "ideal" acknowledgements. I found myself crossing out word after word after word in an earnest, though fruitless, attempt to improve. (One reason to be earnest is when there is only one chance to get it right because the filing date is approaching). The nature of gratitude allows some change, but precludes improvement of my acknowledgements. I realized I was heading toward one of only two possible limits. If I continued on my course, I would end up with no acknowledgements whatsoever. That limit is a possibility, I am sure my committee members could be convinced to sign my thesis (sans acknowledgement) without batting an eye. All the people that read theses could receive all the information they need without reading someone's acknowledgements. On the other hand, there is a certain amount of ridiculousness in removing the acknowledgement of a thesis. I do have gratitude to express even though imperfectly. In spite of my frivolity here, my gratitude is deep and sinceze. I must, therefore, grit my teeth as best I can, as long as I can or as long as I need to, and choose the other limit. That limit is to print my 、 acknowledgements as they stand, with no further alteration. A few might find them interesting; at the very least, someone else coming along after me can read them (just as 
I read the acknowledgements of my predecessors) and decide what they will keep and what they will throw out when they write their own acknowledgements. Also. I have learned something from writing these. I hope someday, after I have aged, I can read this snapshot of my feelings and appreciate the change in my perceptions.

This work was supported by the Director, Office of Energy Research, Office of Basic Energy Sciences, Chemical Sciences Division of the U.S. Department of Energy under Contract Number DE-ACO3-76SF00098. 
TABLE OF CONTENTS

page

Chapter I. Introduction 1

I. The Reaction in Perspective 3

A. Gas Phase Vibrationally Activated 3 Reactions

B. Liquid Water Reaction 6

References 14

Chapter II. Experimental 16

I. Introduction to System Operation . 16

II. Liquid System 21

A. Components 21

B. Assembly 26

1. Cell 26

2. Thermocouple 26

3. Ion Exchange Column 27

4. Interconnection 27

C. Cleaning 28

D. Liquid System Operation 31

1. Cell Constants 31

2. Feedstock $\mathrm{H}_{2} \mathrm{O} \quad 32$

3. Establishing Proper Conditions 33

4. Isotopically Mixed System 35

a. Feedstock $\mathrm{D}_{2} \mathrm{O} \quad 35$

b. Column Deuteration $30^{\circ}$

c. Isotopic Analysis $\quad 36$ 
III. Excitation Sources

IV. Electronics

A. Conductivity Monitor

B. Triggered Differential Amplifier

52

C. Digitizer

57

D. Timing Electrorics

58

E. Electronics Performance

v. Absorption Spectra 66

$\begin{array}{ll}\text { References } & 70\end{array}$

Chapter III: Analysis 71

I. Laser Induced Dissociative Ionization 71

Quantum Yields

A. Concentration of Absorbed Photons 74

1. Concentration of Abscrbed Photons from the Conductivity Step

2. Concentration of Absorbed Photons from the Absorption Spectra

B. Concentration of Reactive Events

c. Conductivity from Signal Voltages

II. Thermal Recombination Rate

III. Discussion of Error

A. Conductivity-based Quantum Yields 90

B. Absorbance-based Quantum Yields 96 
Chapter IV. Results 100

I. Laser-induced Reaction 100

II. Thermal Recombination Reaction 124

References 134

Chapter v. Discussion 135

I. Thermal Recombination Reaction 135

II. Laser Reaction 142

A. Model-independent .Interpretation 145

1. Nature of the Laser-induced Reaction 145

2. Wavelength Dependence of the Quantum 147 Yield in $\mathrm{H}_{2} \mathrm{O}$

3. Quantum Yield Temperature Dependence 151

4. Hydrogen Mole Fraction Dependence 151 of the Quantum Yield and the Wavelength Dependence in Nearly Pure $\mathrm{D}_{2} \mathrm{O}$

B. Quantum Yield Model

1. Probability of Ion Pair Separation 158

2. $\mathrm{k}_{1 \mathrm{a}} / \mathrm{k}_{-1 \mathrm{a}} \quad 165$

3. $1 /\left(k_{1 a}+k_{2}\right) \quad 169$

C. Application of the Quantum Yield Model 173 to Results

1. Quantum Yield vs. Hydrogen Mole 174 Fraction

2. Quantum Yield vs. Temperature 195

3. Quantum Yield vs. Photon Energy 202

$\begin{array}{ll}\text { References } & 214\end{array}$ 
Chapter VI. Conclusions 219

I. Thermal Recombination Reaction 219

II. Vibrationally Activated Reaction 219

Appendix A. Independence of Signal with Laser 222

Position and Intensity Profile

Appendix B. Quantum Yield Program 226

Appendix C. Recombination Distance Program 237

Appendix D. Non-statistical Effects in Overtone 242

Photochemistry

References $\quad 245$

Appendix E. Further Work 246

References $\quad 251$ 


\section{CHAPTER I. INTRODUCTION}

A complete understanding of chemical kinetics requires exploration of all possible physical conditions in which reactions occur. One class of reactions particularly resistant to study are the reactions of vibrationally activated species in condensed phase. This is in contrast to gas phase reactions where vibrational activation is commonplace. 1 An arsenal of methods including a pipette and stopwatch, stopped flow, flow tube, equilibrium perturbation, and NMR exist for study of liquid phase reactions of molecules with a Boltzmann distribution of internal energy. 3 However, they are unable to produce vibrational activation. Perhaps the most clearcut example of a vibrationally activated reaction, first described by Goodall and Greenhow ${ }^{2}$, is the proton transfer reaction of ground electronic state liquid water induced by single photon overtone absorption

$$
\mathrm{H}_{2} \mathrm{O} \stackrel{\mathrm{h} \nu}{+} \mathrm{H}_{2} \mathrm{O}^{*}+\mathrm{H}^{+}+\mathrm{OH}^{-} \text {. }
$$

One other method besides single photon overtone excitation has been used to produce vibrational activation of molecules in liquids. When Franck-Condon factors are favorable, photoexcitation can produce molecules with both electronic and a variable amount of vibrational energy determined by the excitation wavelength. Typically, effects resulting from the vibrational energy of the electronically excited reactant are difficult to observe. 
Quantum yields of liquid phase photochemical reactions are independent of excitation wavelength, and therefore of the level of vibrational excitation. A portion of the total product yield is formed during the time the reactant is vibrationally activated. But in most reactions, vibrational relaxation competes so effectively with reaction that the total yield is dominated by reactions originating from the ground vibrational state of the excited electronic level. Thus, any wavelength dependent yield arising from vibrational excitation is swamped by the yield from vibrationally relaxed molecules. Turro et al. ${ }^{4}$ recently reviewed a few exceptions (none proton transfer reactions) possessing wavelength dependent organic photochemical yields. But even these examples have problems of interpretation. It is difficult to distinguish a wavelength dependent mechanism involving participation of higher electronic states $i S_{n}$ or $T_{n}$ with $n>1)$ from a mechanism caused by vibrational excitation of $s_{1}$ or for that matter $s_{0}$.

There are several advantages when single photon overtone excitation is used for activation. Since absorption is on the potential energy surface of the ground electronic state, only two reactive processes can occur. They are either the vibrationally activated reaction or additional thermal reaction caused by the temperature jump resulting from photon absorption. These two processes are well separated in time, with the 
vibrationally activated molecules reacting on a timescale fast compared to relaxation of the thermal equilibrium. There is no possible interference from the reaction of vibrationally relaxed but electronically excited molecules as there is in the organic photochemical reactions described by Turro. 4

The Goodall and Greenhow ${ }^{2}$ experiments were the first to use single photon overtone excitation to vibrationally activate a reaction in either the gas or 1 iquid phase. They excited the $v=30-H$ stretch overtone at $9399 \mathrm{~cm}^{-1}$. In a subsequent paper, quantum yields were measured as a function of temperature at four fixed wavelengths. 5 This thesis describes and discusses quantum $y$ ields for the liquid water dissociative ionization as a function of temperature, wavelength, and isotopic composition. In addition, thermal recombination rates and distances are given as a function of temperature and isotopic composition.

The introduction will put this work in perspective by relating it first to other gas phase vibrationally activated reactions, and then briefly to previous work on thermal, Boltzmann distribution proton transfer reactions. This work establishes a conceptual framework for description and interpretation of reactions of vibrationally activated species in condensed phase. Unfortunately, disadvantages inherent in the $\mathrm{H}_{2} \mathrm{O}$ proton transfer system prevent a quantitative physical interpretation of results. A better experimental system could be amenable to a more quantitative interpretation (see Appendix E). 
I. The Reaction in Perspective

A. Gas phase vibrationally activated reactions

Most vibrationally activated gas phase reactions involve chemical activation of isolated molecules.

A statistical (RRKM) theory of dissociation works well for isolated gas phase molecules. The liquid water reaction more closely resembles some gas phase experiments performed to demonstrate non-statistical behavior. An overview of experiments performed will be discussed.

Kineticists find it necessary to explore the limits of applicability of statistical theories. The goal is not simply to demonstrate non-statisticality, but to understand why exceptions occur. Although it is reasonable to expect the occurrance of non-statistical unimolecular reactions, 6 few examples have been demonstrated. Most approaches use some type of non-statistical excitation (chemical or photoactivation) and look at the reaction rate or reaction products either on a timescale short compared to the intramolecular relaxation time (high pressures with rapid quenching rates) or use a system where a non-statistical reaction is expected even at long times (extremely low activation energies). Several approaches were recently reviewed. 7 The approaches that examine low activation energy processes include one that is technically a bimolecular reaction. It is the addition of fluorine to ethylene 8,9 or allene ${ }^{10}$ isolated together in rare gas matrices. These 
reactions have energy bariers low enough that activation occurs by vibrational excitation in an energy range low enough for nearly harmonic vibrations. There is no intramolecular vibrational relaxation in a harmonic system so a non-statistical distribution of vibrational energy is expected. Quantum yields are observed for excitation of some modes that do not monotonically increase with energy. Unimolecular reactions of matrix isolated species have been considered in a recent review. They show no mode specific behavior. 11

Another type of low activation energy unimolecular decomposition is dissociation of gas phase van der waals molecules. The internal energy distribution of the dissociation products is non-statistical 12,13

Most demonstrations of non-statistical behavior at high pressures involve chemical activation. The function of the high pressure is to quench all but the initial reactions that occur: immediately following excitation, before intramolecular relaxation is complete. One inter. esting example involves methylene addition to a fluorinated alkene to form a symmetric bicyclopropyl, fluorinated alkane with only one cyclopropyl ring initially activated. At high pressures, decomposition of the initially activated ring predominates with a reduced yield from the symmetric non-activated ring. Other reactions where regions of a large molecule physically separated from the reaction coordinate are chemically activated also 
show a pressure dependent product distribution indicative of incomplete intramolecular vibrational relaxation interrupted by collisions. $7,15-18$

A few attempts to observe non-statistical gas phase reactions use single photon overtone excitation for activation. Several molecules show RRRM behaviorl9 and two, allyl isocyanide 20,21 and t-butylhydroperoxide22, have experimental behavior interpreted as showing non-statistical effects. The t-butylhydroperoxide reaction is similar to the chemical activation experiments already described in that high pressures make the activation occur near the reactive site of a large molecule. The oscillator strength of the excited overtone transition is assigned to the local mode $0-H$ stretch of the dissociating $\mathrm{O}-\mathrm{OH}$. The experimental results are consistent with the presence of a rapid non-statistical dissociation that is not quenched along with a "normal" RRKM reaction that is quenched at high pressures. The allyl isocyanide reaction shows a reaction rate not in agreement with RRKM theory at all pressures tested.

The rull potential of activation by single photon overtone absorption to test RRKM theory has not been exploited. (See Appendix D)

\section{B. Liquid Water Reaction}

We now return to the liquid water reaction. The 
liguid structure consists of a kind of "supermoleculecomposed of an extensive network of individual hydrogen-bonded water molecules. The overtone spectrum can be understood in a local mode model23 with absorption strength caused by excitation of motions with a large component of $\mathrm{O}-\mathrm{H}$ stretch motion of an individual oscillator. Following of stretch excitation, the ensuing energy relaxation is not confined to a single water molecule, but can spread to hydrogen-bonded neighbors of the "supermolecule". Then there is competition between activation of the reactive precursor (perhaps the four-molecule inear chain proposed for the thermal reaction 24 ) and randomization (relaxation) into the rest of the "supermolecule". A purely statistical, RRRM treatment of the entire "supermolecule" does not work. If energy randomization in the "supermolecule" was complete before any product was formed, then no laser induced reaction would be observed.

In several respects, the water reaction is similar to the non-statistical reactions just described. The activation energy is relatively low, being intermediate between typical statistical gas phase unimolecular reactions and non-statistical van der Waals molecule dissociations or bimolecular reactions of matrix-isolated species. Also, as in the high pressure chemical activation experiments, the observed reaction is in competition with "intramolecular" relaxation. The analogy is strongest for the unimolecular reaction of 
t-butyl hydroperoxide. As in $\mathrm{H}_{2} \mathrm{O}$, activation by excitation of $\mathrm{OH}$ stretch overtones is followed by the competition of two processes. In the t-butyl hydroperoxide reaction, activation of the non-statistical O-OH dissociation competes with statistical energy randomization into the entire molecule.

The question remains, "What kind of description will be useful for characterization of the liquid water reaction?" Once again it is instructive to consider analogous gas phase reactions. Several authors have suggested that description of non-statistical gas phase unimolecular reactions will require some combination of RRRM and slater theories.25 slater theory describes the reaction rate when the energy in each mode is defined and does not change with time. This situation will prevail in the limit of weakly coupled modes. In other words, if the quantum state of each mode is adiabatic (does not change during the reaction), Slater theory describes the reaction. The importance of a mode to the rate calculation depends upon its projection on the reaction coordinate; where the reaction coordinate is defined as the lowest energy pathway from reactant to product. If a mode has any projection upon the reaction coordinate, it will be designated an "active" mode. If a molecule has enough vibrational excitation in each active mode to reach the global critical configuration, then the minimum potential energy of a molecule passing 
from the reactant to the product manifold is the potential energy of this critical configuration. If some modes a not contain enough energy to reach the global critical configuration, then reaction can still occur, but the minimum potential energy is at a local saddle point instead of the eritical configuration of the global saddle point. A portion of configuration space which includes the critical configuration of the global saddle point is excluded by the defined quantum state of each mode.

For a statistical RRKM, unimolecular reaction of an isolated molecule, the energy is confined to the excited molecule. Strong mode coupling causes the energy to be rapidly shuttled among the available modes. The number of strongly coupled modes used in an RRKM calculation is equal to the number of modes in the molecule. Since all active modes are strongly coupled, and can take on any energetically allowed quantum state, the minimum potential energy of a reacting molecule is defined by the global saddle point energy minimum in the configuration space of the entire molecule. In other words, all modes of the molecule are strongly coupled, and all active modes are strongly coupled.

To sumarize: in RRRM theory, a luode is important if it is strongly coupled to the modes containing vibrational excitation. In slater theory, a mode is important if it contains vibrational energy and is "active". 
One promising conceptual picture for non-statistical gas phase or for a vibrationally activated liquid phase process is provided by first breaking the modes of the system into active and non-active modes, and then once again into modes strongly-coupled and not-strongly-coupled to the modes that are vibrationally excited. The four possible types of mode are shown below.

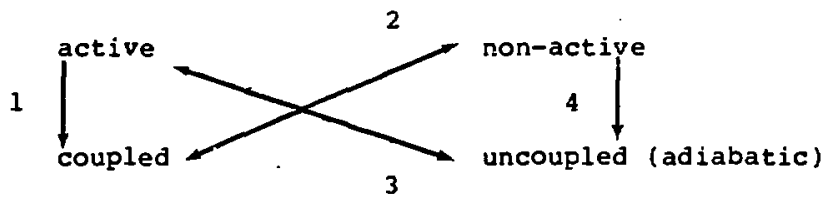

Non-active uncoupled modes (4) can be ignored. The major difference between a statistical unimolecular reaction and the situation described here is that some active modes can be uncoupled (3). Thus, the energy barrier is greater than the global saddle point. A description of the reaction rate by RRKM theory with the number, and frequency of modes determined by whether a mode is strongly coupled, and the energy barrier determined by the number of strongly-coupled, active modes will combine the relevant aspects of both RRKM and slater theory. Thus reaction rates in condensed phase depend on the details of the energy flow; in other words, it depends on which modes are strongly coupled to excited modes. No general guideline as to the identity of each mode can be provided, but it is expected that a reactive gas 
phase molecule might show a higher energy barrier and/or smaller number of stro:-jly coupled modes when vibrationelly activated in a liquid. Conceivably, if the relaxation path, critical configuration, and energy barrier were known, the reaction rate could be calculated quantitatively. However, there are several problems inherent in the $\mathrm{H}_{2} \mathrm{O}$ system which prevent a quanticative

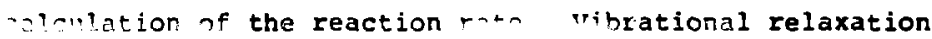
rates and pathways, and ion pair separation probabilities are not known. Therefore, only quantum yields can be datermined, not reaction rates. Also, the identity of the reactive species and of the "active" modes is not known. Appendix E suggests other potertiai work including some systems where a quantitative treatment may be possible.

The gas phase experiments are performed by measuring product quantum yields as a function of pressure following a C.W. photolysis. Long irradiation times are required. This necessitates use of reactants with an activation energy high enough to prevent interfering dark reaction products.

There are major experimental differences between the gas phase and liquid phase experiments. In a liquid, the combination of low cross section for high overtone excitation and lower quantum yields resulting from rapid intermolecular relaxation makes product detection 
following a C.W. photolysis in liquids very difficult. Instead, pulsed excitation and transient conductivity measuretuents are used to generate and detect ionic products. Low activation energy processes can be studied because the presence of thersally generated product does not interfere with measurement of the laser generated transient products.

The liquid water proton transfer reaction is also interesting for several reasons not related to fundamental chemical kinetics. It is important to increase our understanding of liquid water and of proton transfer reactions. Water is the most important solvent in chemistry, and biology. Proton transfer reactions have a similar importance. Acid- and base-catalyzed reactions are important in the synthesis of commercial chemicals, and proton transfers play a role in many biologically important enzyme reactions. The liquid water ionization, being the simplest aqueous proton transfer imaginable is an obvious candidate for study.

The vibrationally activated reaction offers a new way to perturb the $\mathrm{H}_{2} \mathrm{O} \rightrightarrows \mathrm{H}^{+}+\mathrm{OH}^{-}$equilibrium and potentially offers new information on the reaction and relaxation of the ion pair precursor not obtained in equilibrium relaxation measurements. The recombination of $\mathrm{H}^{+}$and $\mathrm{OH}^{-}$following equilibrium perturbation is diffusion controlled24, thus no kinetic information is obtained for the recombination of the ion fair by varying 
the temperature or isotopic composition. The actual ion pair recombination is masked by the diffusion. In contrast, the vibrationally activated reaction is dependent upon both the reaction rate to form the ion pair, the recombination rate, and the diffusion rate. Thus details of reaction, relaxation, and recombination are to some extent unmasked.

The remainder of the thesis describes (A) the experimental apparatus, (B) analysis of data, (C) quantum yield results as a function of temperature, excitation wavelength, and isotopic composition, thermal recombination rates as a function of temperature and isotopic composition, (D) a discussion and qualitative interpretation of the results, and (E) conclusions. Several appendices are included to clarify some points, to add some details, and to stimulate thought. 
1. "Unimolecular Reactions", P.J. Robinson and R.A. Holbrook, Wiley, Lonsion (1972).

2. D.M. Goodall and R.C. Greenhow, Chem. Phys. Lett. 9, 583 (1971).

3. J.E. Crooks in "Comprehensive Chemical Rinetics", v. 8, C.H. Bamford and C.F.H. Tipper, eds., Elsevier, Ansterdam (1977).

4. N.J. Turro, v. Ramamurthy, w. Cherry, and w. Farneth, Chem. Rev. 78, 125 (1978).

5. B. Rnight, D.M. Goodall, and R.C. Greenhow, J. Chem. Soc. Faraday II 75, 841 (1979).

6. S.A. Rice in "Advances in Laser Chemistry", v. 3 , A.H. Zewail, ed., Springer-Verlag, Berlin (1978).

7. I. Oref and B.S. Rabinovitch, Acc. Chem. Res. 12, 166 (1979).

8. H. Frei, L. Fredin, and G.C. Pimentel, J. Chem. phys. 74, 397 (1981).

9. H. Frei and G.C. Pimentel, J. Chem. Phys. 78, to be published (1983).

10. A.K. Knudsen and G.C. Pimentel, submitted to J. Chem. Phys.

11. M. Poliakoff and J.J. Turner in "Chemical and Biochemical Applications of Lasers", v. V, C.B. Moore, ed.. Academic Press, New York (1980).

12. D.H. Levy, L. Wharton, and R.E. Smalley in "Chemical and Biochemical Applications of Lasers", v. 2, Academic Press, New York (1977). 
13. D.H. Levy, L. Wharton, and R.E. Smalley, Acc. Chen. Res. 10, 139 (1977).

14. J.D. Rynbrandt and B.S. Rabinovitch, J. Phys. Chem. 75, 2164 (1971).

15. A.-N. Ko and B.S. Rabinovitch, Chem. Phys. 30, 361 (1978).

16. J.F. Meagher, K.-J. Chao, J.R. Barker, and B.S. Rabinovitch, J. Phys. Chem. 78, 2535 (1974).

17. F.C. Wolters, B.S. Rabinovitch, and A. -N. Ko, Chem. Phys. 19, 65 (1980)

18. A.B. Trenwith, B.S. Rabinovitch, and F.C. Wolters, J. Chem, Phys. 76,1586 (1982).

19. J.M. Jasinski, J.K. Frisoli, and C.B. Moore, Discuss. Faraday Soc., submitted.

20. R.V. Reddy and M.J. Berry, Chem. Phys. Lett. $\underline{66}$, 223 (1979).

21 R.V. Reddy and M.J. Berry, Faraday Discuss. Chem. Soc. 67,188 (1979).

22. D.W. Chandlex, W.E. Farneth, and R.N. Zare, J. Chem. Phys. 77, 4447 (1982).

23. A.C. Tam and C.K.N. Patel, App. Opt. 18, 3348 (1979).

24. M. Eigen and L. DeMaeyer, Proc. Roy Soc. (London), Ser. A 247, 505 (1958).

25. R.D. Levine, Adv. Chem. Phys, 47, part 1, 239 (1981). 


\section{CEAPTER II. EXPERIMENTAL}

The experimental goal is to measure the transient conductivity of a sample of pure water following laser excitation of $\mathrm{O}-\mathrm{H}$ stretch overtones. The experimental apparatus consists of (1) a liquid system used to contain transport, purify and monitor the water, (2) an optical excitation source, and (3) electronics used to monitor and record the time dependent conductivity. A Cary 14 spectrometer is used to record absorption spectra. This section gives an introduction to the operation of the apparatus, tinen gives the details of the three subsystems.

\section{Introduction to system operation}

The water conductivity is measured by a cell in the $\mathrm{H}_{2} \mathrm{O}$ system along with the triggered differential amplifier, low pass filter, digitizer and averager shown in Figure II-1. The differential amplifier and digitizer are triggered by a pulse generator. After a short delay, a Raman-shifted laser source is triggered. The generated light excites overtones of the water flowing between electrodes of the conductivity cell. Product ions give a transient conductivity recorded by a signal averager. The $\mathrm{H}_{2} \mathrm{O}$ system removes impurity ions by continuousiy circulating the water in a closed loop 
Figure II-1

Transient conductivity changes induced by dissociative ionization of water are produced and measured with this apparatus. 


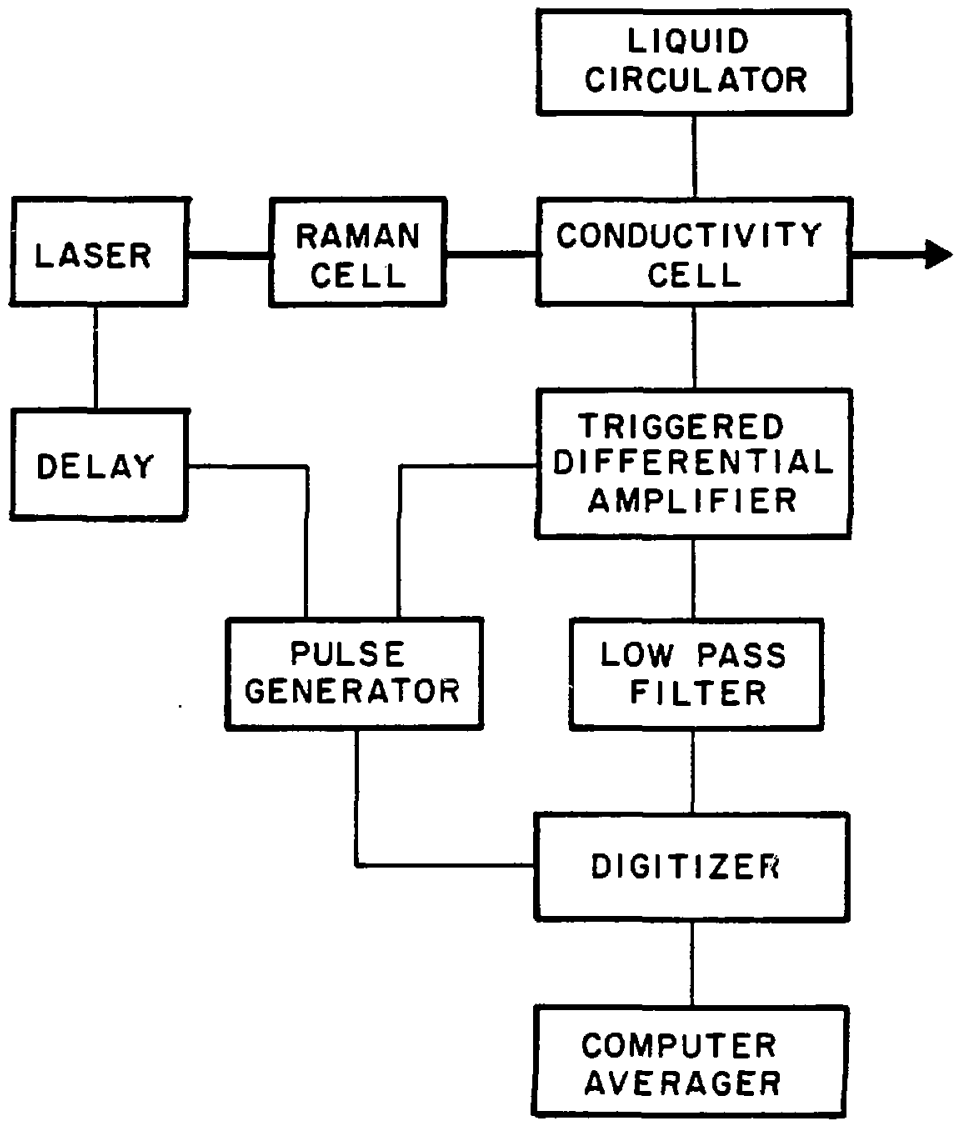

XBL 8212-12403 
Figure II-2

Schematic diagram of the water system. 


\section{Reservoir}

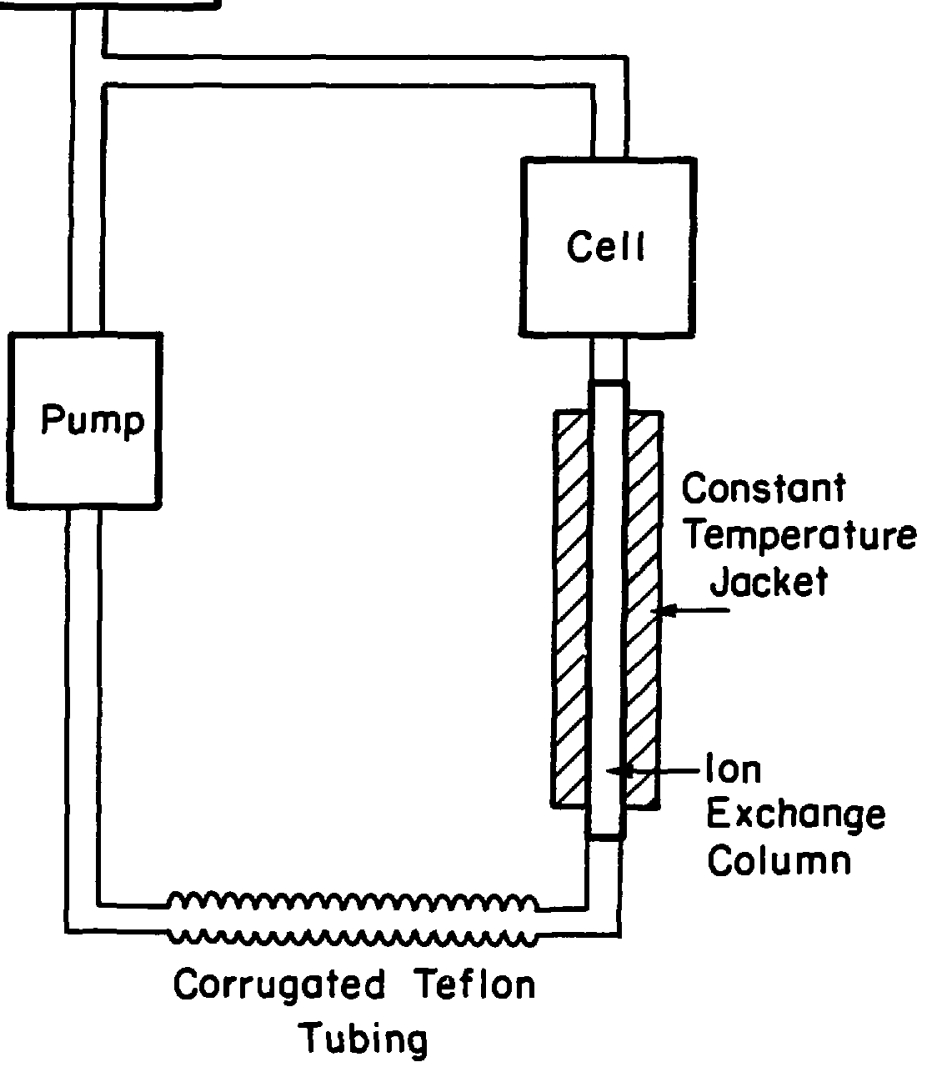

XBL $831-7706$ 
through an Ion exchange colunn inmediately preceding the conductivity cell.

\section{Liquid system}

This section describes the components, the assembly. the cleaning, and the operation of the water system, Figure II-2, containing $\mathrm{H}_{2} \mathrm{O}, \mathrm{D}_{2} \mathrm{O}$, or isotopic mixtures.

The best closed loop circulation system was constructed of quartz, Pyrex, FEP Teflon, PTFE Teflon, and silicone rubber. Other trial systems will be mentioned.

\section{A. Components}

The components of the $\mathrm{H}_{2} \mathrm{O}$ system consist of a water reservoir, a pump, an ion exchange column with constant temperature jacket, a thermocouple, and a conductivity cell.

A $225 \mathrm{ml}$ Pyrex bulb is used as a water reservoir during system $f i l l$ and evacuation/degassing. It supplies a Cole palmer Masterflex pump. The pump uses a model 7545-00 drive unit with a 7017 peristaltic pump head using 6411-47 silicone tubing. The tubing is $0.25^{n}$ inside diameter (ID) and 0.379" outside diameter (OD). An $18 \mathrm{~cm}$ piece of 1/4" SGA Scientific \#R8428-14 corrugated, flexible teflon tubing is connectd to the pump outlet. The ion exchange column is a $78 \mathrm{~cm}$ length of $8 \mathrm{~mm} O D$, 
7 m ID, TEE Elastofluor tubing partially filled with Rohm . Haas Amberlite MB-l mixed bed ion exchange resin. Column supports are made of $0.21 \mathrm{~mm}$ mesh Teflon spectramesh grid \$146464 from Spectrum Medical Industries. The grid is stretched over a few millimeters-length of polytetrafluoroethylene, PTFE tubing and then inserted in the column. Larger diameter vycor glass and polyethylene columns with glass wool column supports were tested with satisfactory results. The ion exchange column is jacketed with a concentric 1-1/8" diameter copper tube. Brass end caps with Viton o-rings seal the copper jacket to the teflon ion exchange column. The wacer temperature is adjusted by circulating methanol in the jacket. The methanol is pumped with a Lauda model $K-4 / R$ temperature controller by Brinkmann Instruments. The absolute water temperature is measured with a copper-constantan thermocouple with a water-ice reference. The conductivity cell shown in Figure II-3 is machined

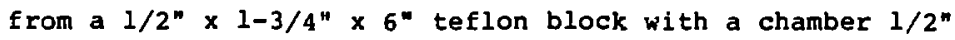
$x 4^{\prime \prime}$. Inner walls are smooth and edges perpendicular to the flow are rounded to minimize water turbulence. The bare platinum electrodes are made of $0.051^{\text {" diameter }}$ platinum wire bent and flattened into a staple with $2.7 \mathrm{~mm}$ $x 9 \mathrm{~mm}$ electrode faces. Electrode pairs are located 2-1/2" and $3^{\prime \prime}$ downstream from the cell inlet to allow inlet turbulence to damp before reaching the electrodes. Quartz windows $1 / 8^{\prime \prime}$ or $1 / 16^{\prime \prime} \times 1^{\prime \prime} \times 6^{\prime \prime}$ are sealed with viton $0-r i n g s . ~ A ~ 1 / 16^{*}$ rubber pad is sandwiched between 
Figure II-3

Scale drawing of conductivity cell and electrodes. A 6" $x$ 1-3/4." $x$ 1/8" stainless steel plate with a $1-3 / 4^{\prime \prime} x$ $1 / 2^{\text {" }}$ opening facing the electrodes screws into the cell body and forces a window against the o-ring. The plate and window are not shown. 


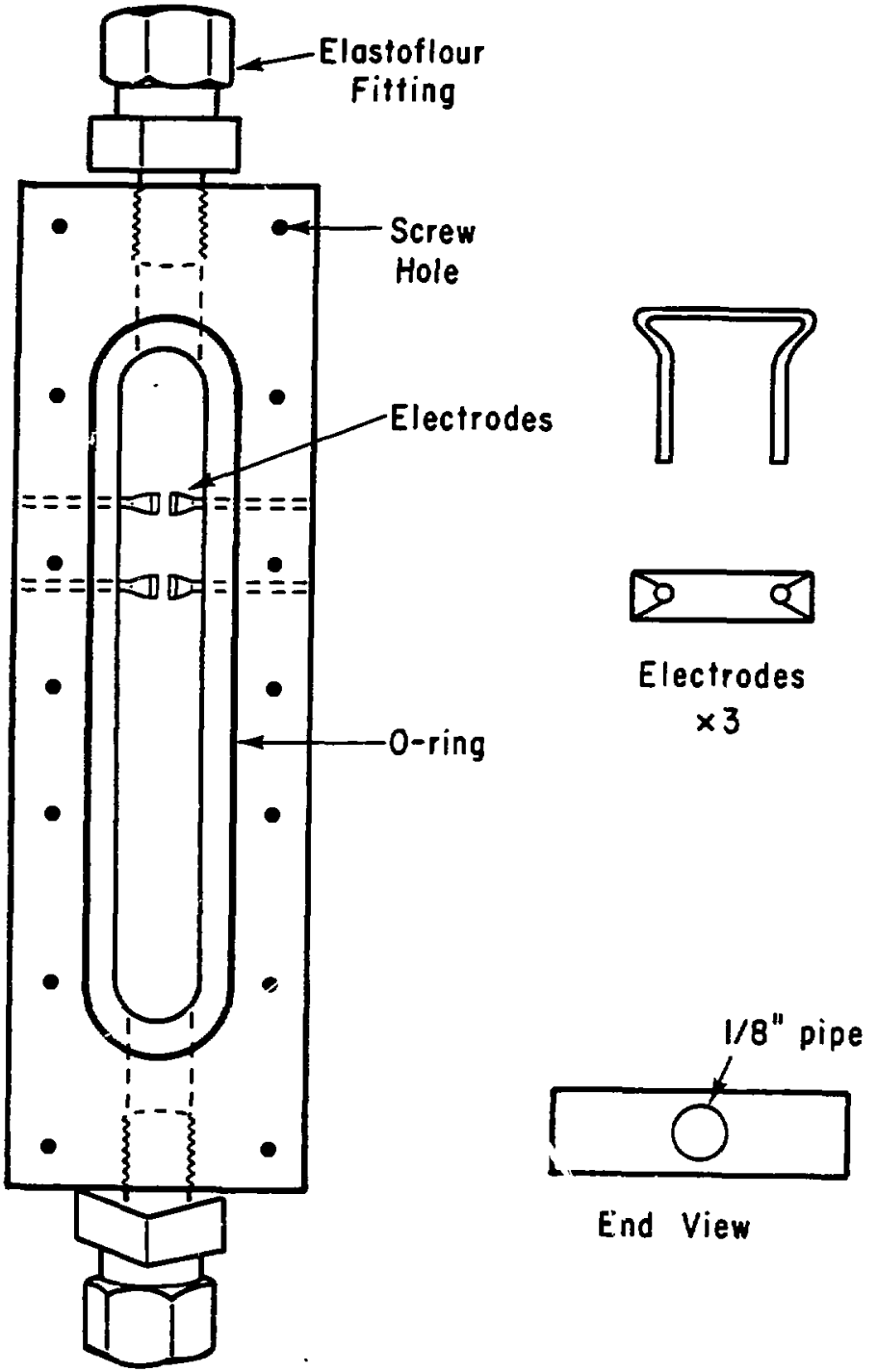

XBL 8212-12402 
the window and a $1 / 8^{*} \times 6^{\prime \prime} \times 1-3 / 4^{\prime \prime}$ stainless steel retaining plate which screws into the teflon cell.

Several other cells were used for some $\mathrm{H}_{2} \mathrm{O}$ guantum yield measurements. A teflon sell with epoxied guartz windows and epoxied platinum staple electrodes was used for measurements taken with a CMX-4 dye laser pumped optical parametric oscillator. Also, two laminar fluid flow cells were used with a Quanta Ray $N d^{+3}:$ YAG pumped dye laser system. One was made of $3 \times 9 \mathrm{~mm}$ rectangular cross section quartz tubing with platinum foil electrodes epoxied to ground quartz inserts. Long times we:e required to desorb ions from ground glass surfaces of. the quartz isserts after exposure to $\mathrm{KCl}$ conductivity solutions used to determine cell constants. Another cell was made from quartz strips epoxied to form a rectangular channel $1 \mathrm{~mm} \times 2 \mathrm{~mm} \times 300 \mathrm{~mm}$. Electrodes were produced by laminating $9 \mathrm{~mm} \times 14 \mathrm{~mm}$ strips of 0.001 " platinum foil to the inner wall of the rectangular cell with FEP teflon film. The electrodes were loca: $25 \mathrm{~cm}$ from the cell inlet so that turbulence created at the cell inlet is damped before reaching the electrodes. Torr-Seal epoxy from Varian was selected for its low interaction with $\mathrm{H}_{2} \mathrm{O}$. This cell was discarded after delamination of an electrode. Platinum electrodes vacuum deposited directly on quartz disintegrated on contact with water. However, platinum can be vacuum deposited over an underlayer of titanium or niobium about $200 \mu \mathrm{m}$ in thickness ${ }^{1}$. Metal oxides, $\mathrm{Bi}_{2} \mathrm{O}_{3}$ and $\mathrm{Pbo}$, 
have been used for some applications reguiring transparent electrodes 2 .

\section{B. Assembly}

System assembly is done in two parts. First, the celi, thermocouple, and ion exchange column were assembled. Then, the components are interconnected primarily with $8 \mathrm{~mm}$ Elastofluor fittings and PTFE tubing.

\section{Cell}

Electrical connection must be made to the electrodes before attaching them to the cell body. Copper wire is soldered into holes concentrically drilled in the base of the electrode arms. Then the arms are gripped with pliers and forced into undersize holes drilled in the teflon cell. Aluminum foil is used to protect the arms during insertion. A uniform interelectrode distance of $2 \mathrm{~mm}$ is obtained by using two removable glass microscope slides as spacers.

\section{Thermocouple}

The thermocouple is encased in a sealed $4 \mathrm{~mm}$ OD Pyrex tube pressure fitted within a concentric $3.5 \mathrm{~cm}$ length of $8 \mathrm{~mm}$ oD teflon rod. The rod mounts in an Elastofluor fitting. The immersed length is adjusted to about $6.5 \mathrm{~cm}$. 
The thermocouple tip lies $7.8 \mathrm{~cm}$ downstream from the final electrode pair of the conductivity cell.

\section{Ion exchange column}

Rohm and Haas Amberlite MB-1 mixed bed resin is used in the ion exchange column. Trace amounts or amine and styrene decomposition products are leached from the resin by soaking in high purity water for several days. Ten to fourteen $m l$ of resin are poured into the column in several. small portions to minimize separation of cationic and anionic forms of the resin caused by a higher settling rate for the denser cationic form. A larger resin volume does not significantly improve the ionic purity of the $\mathrm{H}_{2} \mathrm{O}$ and will impart trace amounts of organic impurities at a higher rate. Higher pressures at the output of the pump decreases silicone tubing lifetime. Bio Rad AG501-X8 ion exchange resin columns were not used because they produced $\mathrm{H}_{2} \mathrm{O}$ with twice the conductivity of the column described.

\section{Interconnection}

Elastofluor fittings can not be used to connect the silicone pump tubing or the fluorinated ethylene-propylene, FEP, Teflon corrugated tubing to the Elastofluor PTFE tubing. The silicone tubing is simply slipped over and clamped onto the Elastofluor tubing with metal tubing clamps. 
The FEP tubing is attached as shown in Figure II-4. The smooth ends of the FEP tubing and the Elastofluor tubing are slipped over a snug-fitting $7 \mathrm{~mm} O \mathrm{OD}, 3.2 \mathrm{~cm}$ length of PTFE tubing insert. The assembly is covered with an FEP sleeve under PTFE heat shrink tubing, SGA catalog No. R8428-5, and is heaced with a heat gun above the approximate $270^{\circ} \mathrm{C}$ meiting point of the FEP sleive. The FEP Teflon melts forming a strong bond to the PTFE tubing. Care must be taken not to heat the portion of the FEP corrugated tube beyond the PTFE insert.

\section{c. cleaning}

The Tef_on, quartz, Pyrex, and silicone rubber parts of the system must be cleaned to prevent contamination of the high purity circulating water.

The Teflon cell body, Elastofluor fittings, and spectramesh column supports were cleaned by soaking in aqua regia and then rinsing in water for about 5 hours. The PTFE tubing and flexible FEP tubing were assembled with the clean Elastofluor fittings and filled with concentrated nitric acid for about 5 hours. The system was then rinsed and filled with water for 4 hours. Quartz windows were cleaned with methanol then soaked in an aqueous 28 sodium hydroxide, 18 sodium EDTA solution to remove adsorbed ions. The Pyrex water reservoir and 
Figure II-4

Attachment of FEP corrugated tubing, A, to Elastofluor PTFE tubing, B, is shown. The corrugated tube must be slipped on the PTFE insert, $C$. Then the heat shrink tubing, $E$, and FEP sleeve, $D$, are heated to seal the FEP. 
30

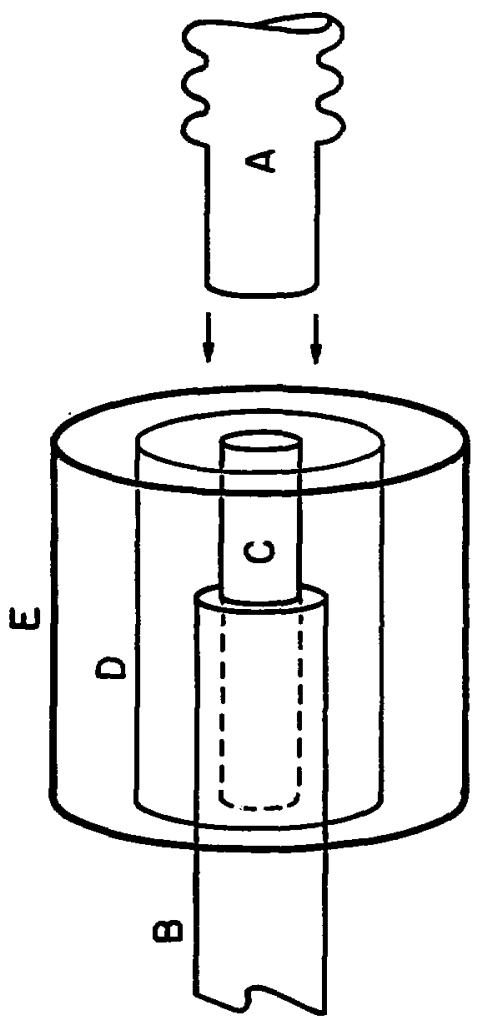


thermocouple well are boiled for several minutes in dilute nitric acid.

Electrodes were cleaned before assembly by immersion in 1 part nitric acid to 3 parts hydrochloric acid followed by passage of 1 amp of current for two minutes through the positively charged electrode. A platinum wire served as the negative electrode. Silicone tubing for the peristaltic pump was cleaned by rinsing with methanol. followed by immersion in boiling water which is allowed to cool to room temperature.

preferred materials and cleaning procedures for highpurity systems are discussed in reference ( 3 ).

D. Liquid system operation

Operation of the liquid system with $\mathrm{H}_{2} \mathrm{O}$ includes the following steps: (1) cell constant determination, (2) preparation of feedstock water, and (3) establishing the proper temperature, purity and flow in the circulating water. System preparation and isotopic analysis with $\mathrm{H}_{2} \mathrm{O} / \mathrm{D}_{2} \mathrm{O}$ mixtures will also be described.

1. Cell constants

Cell constants for both electrode pairs were determined by measuring the resistance of a $10^{-2}$ molar $\mathrm{KCl}$ solution with an A.C. Wheatstone bridge. The cell is immersed in a constant temperature bath of known temperature and allowed 
to equilibrate. The cell capacitance and resistance is determined by bridge balance. The cell constant, $k$, was found by the equation

$$
\mathbf{k}=T \cdot R
$$

where $R$ is the cell resistance and $R$ is the conductivity of $10^{-2}$ molar KCl $4,1.413 \times 10^{-3}\left[1-1.981 \times 10^{-2}(25-\mathrm{T})\right]$ $\mathrm{ohm}^{-1} \mathrm{~cm}^{-1}$. The cell constant is $0.319 \pm 0.15 \mathrm{~cm}^{-1}$ for both electrode pairs. The cell must be rinsed, then filled with pure $\mathrm{H}_{2} \mathrm{O}$ for a day or more following cell constant determination to remove contaminating potassium and chloride ions.

2. Feedstock $\mathrm{H}_{2} \mathrm{O}$

Feedstock water for the circulating water system came from two sources. One source was a Pyrex and quartz pyrolytic still where the water vapor is passed in an oxygen atmosphere through a $900^{\circ} \mathrm{C}$ quartz tube pyrolyzer. Trace organic impurities are oxidized to $\mathrm{CO}_{2}$ and $\mathrm{H}_{2} \mathrm{O}$ during passage through the pyrolyzer. The condensed $\mathrm{H}_{2} \mathrm{O}$ is then redistilled in a second stage and stored. An oxygen purge prevents atmospheric $\mathrm{CO}_{2}$ from reaching the water. In the second source, water was prefiltered in Barnstead charcoal and ion exchange resin filters, then distilled in a Corning Mega-Pure still. No difference in conductivity is noticed using water from either source. 


\section{Establishing proper conditions}

Water is introduced to the system by filling the expansion bulb. Dissolved $\mathrm{CO}_{2}$ and many air bubbles nust be removed after pouring an ion exchange column and filling the sytem with $\mathrm{H}_{2} \mathrm{O}$. Evacuation of the system using a dry ice/acetone trapped mechanical pump causes the water to boil. Vertical orientation of the ion exchange column eliminates trapping of rising gas bubbles. Several evacuation/repressurization cycles while circulating the water are needed to remove dissolved atmospheric gasses. The system is repressurized with argon to exclude atmospheric $\mathrm{CO}_{2}$ from the system. The peristaltic pump circulates the water by squeezing flexible silicone tubing with moving rollers. The flow rate of about 2.5 $\mathrm{ml} / \mathrm{sec}$ corresponds to about 60 pump revolutions/min or 180 pressure pulses/min. Flow rate changes caused by the pressure variation at the outlet of the pump can produce turbulence in the conductivity cell contributing to low frequency noise in the conductivity signal. These variations are minimized by impeding the fluid flow at the conductivity cell inlet and allowing the system volume between the pump and the flow restriction to expand and contract in response to pressure pulses from the pump. An $18 \mathrm{~cm}$ section of corrugated FEP teflon tubing and to some extent, the flexibility of the silicone pump tubing allow system expansion. The flow restriction is provided by the ion exchange column. 
An alternate technique is to allow the system to expand and contract into a chamber partially filled with a bubble of compressed gas. This method is undesireable because dissolved gas comes out of solution as the pressure is reduced in the ion exchange column and conductivity cell. Bubbles forming or passing near the conductivity cell electrodes produce spurious signals. Water temperature was varied from $50^{\circ} \mathrm{C}$ to about $-0.1^{\circ} \mathrm{C}$ for supercooled $\mathrm{H}_{2} \mathrm{O}$. The stability is $\pm 0.2^{\circ} \mathrm{C}$ over 2 minutes. At $8.5^{\circ} \mathrm{C}$, the water cools a maximum $0.2^{\circ} \mathrm{C}$ while traveling from conductivity cell to thermocouple. The overall error in the absolute temperature measurement is $\pm 0.4^{\circ} \mathrm{C}$. Most tubing, the ion exchange column, and the conductivity cell are insulated with foam zubber or Armstrong Armaflex. Four systems were used to maintain the purity of circulating $\mathrm{H}_{2} \mathrm{O}$ when measuring the wavelength dependence of the quantum yield: (1) an ion exchange column, (2) an ion exchange column plus a $1.2 \mu \mathrm{m}$ and $0.47 \mu \mathrm{m}$ Millipore filter, (3) an activated charcoal column with an ion exchange column plus both Millipore filters, and (4) an ion exchange column plus a 100 watt Hanovia Hg lamp immersion wel15 plus both Millipore filters. A GC/MS of water which was photolyzed longer than 1.5 hours in system (4) showed no detectable organic impurities or less than $5 \mathrm{ppm}$ by weight. The quantum yields measured with each system agreed within experimental error. The simplest 
system, an ion exchange column, was used with $\mathrm{H}_{2} \mathrm{O} / \mathrm{D}_{2} \mathrm{O}$ mixtures and for temperature dependent studies in $\mathrm{H}_{2} \mathrm{O}$. The conductivity of the system ranges between 1028 to 200 of the conductivity caused by the equilibrium concentration of hydroxide and hydronium ions $15.5 \times 10^{-8}$ $o \mathrm{om}^{-1} \mathrm{~cm}^{-1}$ at $25^{\circ} \mathrm{C}$ ). The poorest conductivities were with the quartz cell with ground glass inserts. The all Teflon system typically gives a conductivity $110 \%$ of. theoretical.

4. Isotopically mixed system

The isotopically mixed system is nearly identical to the $\mathrm{H}_{2} \mathrm{O}$ system with the following exceptions. Feedstock $\mathrm{D}_{2} \mathrm{O}$ must be prepared, an ion exchange column deuterated, and isotopic composition of the system analyzed.

4a. Feedstock $\mathrm{D}_{2} \mathrm{O}$

Feedstock $99.88 \mathrm{D}_{2} \mathrm{O}$ (Bio-Rad catalog no. 710-1003 or Aldrich catalog no. 15,188-2) was pyrolytically distilled. The still was pre-deuterated by distilling and then discarding about $10 \mathrm{ml}$ of $\mathrm{D}_{2} \mathrm{O}$ while heating all normally cool regions of the still with a heat gun. After cooling, 200-300 $\mathrm{ml}$ of $\mathrm{D}_{2} \mathrm{O}$ was added to the still and collected after the first stage of distillation. 
4b. Column deuteration

A deuterated ion exchange column was obtained by preparing the column with $\mathrm{H}_{2} \mathrm{O}$, followed by three successive cycles of draining, filling, and mixing with high purity $\mathrm{D}_{2} \mathrm{O}$. Mixing is accomplished by degassing the system at least six times. vigorous boiling during degassing assists mixing. The ratio of the volume of retained water in the drained system to the volume of the filled system is $1: 8$. The mole fraction of hydrogen in the. firal mixture with highest isotopic enrichment was $0.0057 \pm 0.002$. The ionic conductivity was 1108 of the theoretical conductivity of equibrium $\mathrm{D}_{3} \mathrm{O}^{+}$and $\mathrm{OD}^{-}$in the mixture.

Highly deuterated columns with a smaller $D_{2} \mathrm{O}$ expenditure could be obtained by drying the column before exposure to $\mathrm{D}_{2} \mathrm{O}$. Direct vacuum drying or after prerinsing with dioxane or ether produced resin beads with a tendency to disintegrate upon re-hydration.

4c. Isotopic analysis

The isotopic composition of the $\mathrm{H}_{2} \mathrm{O}$ was determined from samples drawn through a Teflon-coated silicone rubber septum with a syringe. Density measurements with a $3 \mathrm{ml}$ pyncnometer are used to determine isotopic enrichment for hydrogen mole fractions greater than 0.10 . Liquid 
phase infrared spectroscopy was used for smaller hydrogen mole fractions. Measured hydrogen mole fractions have an uncertainty of 0.002 at 0.000 mole fraction hydrogen increasing to 0.01 at 0.10 mole fraction hydrogen. The uncertainty is 0.01 over the remaining range.

Infrared spectra with $8 \mathrm{~cm}^{-1}$ resolution were taken on a Nicolet 7199 FTIR spectrometer using a HgCdTe detector, KBr beamsplitter, and Globar source. Liguid samples were taken in $1 \mathrm{~cm}$ path cylindrical quartz cells. Figure II-5 shows the infrared spectrum of three different $\mathrm{H}_{2} \mathrm{O}-\mathrm{D}_{2} \mathrm{O}$ mixtures in the $5600 \mathrm{~cm}^{-1}$ to $6800 \mathrm{~cm}^{-1}$ region. The peak in the 0.003 mole fraction $\mathrm{H}_{2} \mathrm{O}$. spectrum at $6250 \mathrm{~cm}^{-1}$ is a $D_{2} O$ peak, and the shoulder at $6030 \mathrm{~cm}^{-1}$ is an HOD absorption. The shoulder height increa.es linearly as hydrogen mole fraction is increased. To determine isotopic composition, the peak height of a water sample from the liquid system is compared to a calibration based on measurements at $0.011,0.032,0.058$, and 0.140 mole fraction hydrogen.

Higher resolution gas phase spectra of water vapor in equilibrium with the liquid taken with the same instrument in $15 \mathrm{~cm}$ pathlength sapphire window cells could also be used to analyze the isotopic composition. The area of several well-resolved $O H$ and $O D$ stretch lines of HOD and $\mathrm{D}_{2} \mathrm{O}$ can be measured if pressure broadened beyond the instrumental resolution of $0.06 \mathrm{~cm}^{-1}$ by addition of an inert bath gas; 673 torr of $N_{2}$ produces a measured line 
Figure II-5

The infrared spectra of $0.003,0.017$, and a 0.038 mole Eraction hydrogen sample of water are shown by curves $A$, $B$, and $C$, respectively. The peak at $6030 \mathrm{~cm}^{-1}$ is from 4OD. The peak at $6250 \mathrm{~cm}^{-1}$ is from $D_{2} O$. The pathlength is $1 \mathrm{~cm}$. The absolute scale for spectrum $A, B$, and $C$, is $0.0760,0.0804$, and 0.0800 base 10 absorbance units/division. 


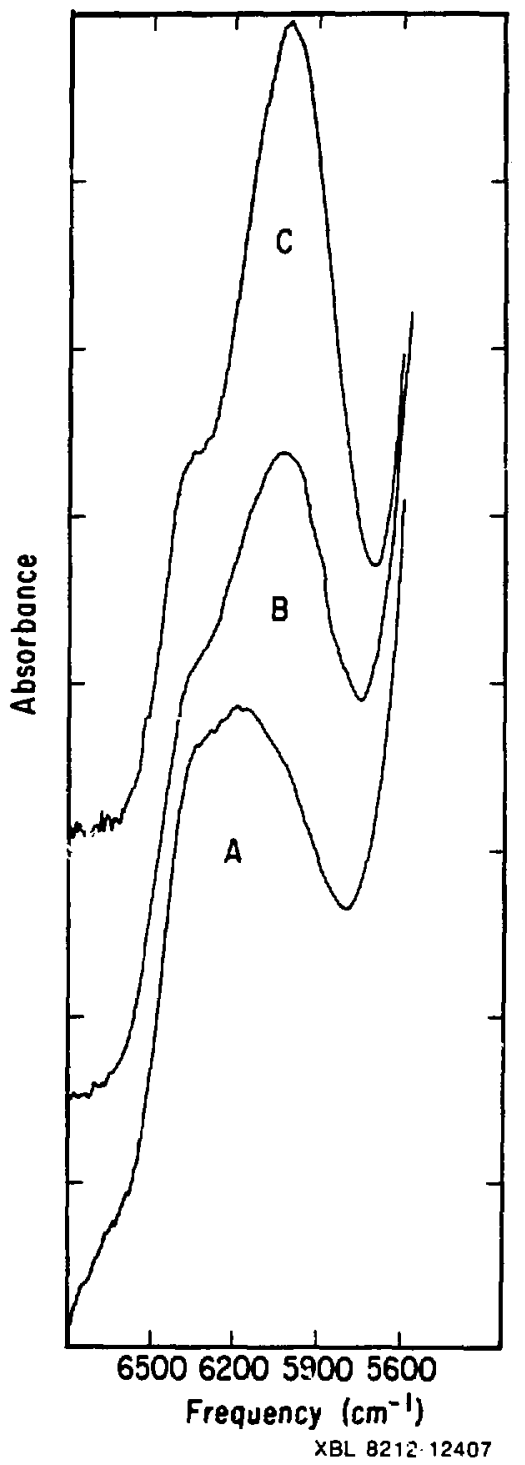


width of $0.28 \mathrm{~cm}^{-1}$. The 1iquid phase spectral analysis was found to be easier and probably more accurate.

\section{Excitation sources}

This section describes generation, wavelength selectior, and alignment of light between 1.41 um and $0.55 \mu \mathrm{s}$ used to excite liquid water.

Preliminary experiments used a Chromatix CMX4/IR with IR-1 mirrors and model $300 \mathrm{~A}$ oven and temperature controller. A Chromatix CMX-4/IR consists of a temperaturetuned, Linbo 3 optical parametric oscillator pumped by a Chromatix CMX-4 flashlamp pumped dye laser. The theory of optical parametric oscillation is described in several review articles 6,7 . Wavelengths between $1.35 \mu \mathrm{m}$ and $0.98 \mathrm{\mu m}$ there generated. The signal and idler beam were separated using a Schott JB-639522 near IR linear variable filter. Up to $200 \mathrm{\mu J}$ pulses of combined signal and idler wavelengths could be generated when pumping with a 6 to $7 \mathrm{~mJ}, 1$ us pulse near $0.59 \mu \mathrm{m}$. Typical powers after the linzar variable filter were 10-30 $\mathrm{JJ}$. Output powers were measured with a Molectron J3-05 pyroelectric detector. Wavelengths were determined with a Bausch and Lomb 600 groves/mm diffraction grating blazed at $28^{\circ} 41^{\prime}$. The grating is calibrated using visible light from the dye laser. The dye laser wavelength is determined with a 1.5 meter Jobin-Yvon monochromator. 
Quantur yields at $9050 \mathrm{~cm}^{-1}$ and $10204 \mathrm{~cm}^{-1}$ agree within 7 and 27 of values measured with the quanta Ray system to be described. Much higher pulse energies were obtained with a Quanta Ray DCR-1, Nd+3:YAG-puoped PDL-1 dye laser that can be stokes shifted in a high-pressure hydrogen or methane Raman cel18. This system is used for all reported quantum yields. Dye laser output is focussed first with a $50 \mathrm{~cm}$ lens external to the 1 m Raman cell. Sixty centimeters from this lens is an internal $6.7 \mathrm{~cm}$ focal length lens supported in a teflon holder. The internal lens produces a second focus which enhances second stokes output. Figure II-6 shows the geometry of the Raman generator. The desired output wavelength is selected with a quartz prism, Corning 7-56 or Corning 7-69 filter. Power is optimized by adjusting the Raman cell gas pressure and divergence of the dye laser. Table II-l shows optimum conditions at several output wavelengths.

The dye laser wavelength is checked with a Beck Wavelength Reversion spectrometer. Reported wavelengths are accurate to 1 nanometer. Near infrared frequencies are calculated from the dye laser frequency and the Raman frequency of the gas in the Raman cell. Hydrogen produces a frequency change of $4155 \mathrm{~cm}^{-1}$ per shift. Methane produces a frequency change of $2917 \mathrm{~cm}^{-1}$ per shift. The typical average light flux at the cell is $20 \mathrm{MW} \mathrm{cm}^{-2}$ in a spot $1.5 \mathrm{~mm}^{2}$. This corresponds to an $8 \mathrm{nsec}$ pulse, with a 
Figure II-6

Excitation source and Raman shifter geometry. The external lens is a $50 \mathrm{~cm}$ F.L. quartz lens. The internal lens is a $1 \mathrm{~cm}$ diameter $6.7 \mathrm{~cm}$ F.L. glass lens. 


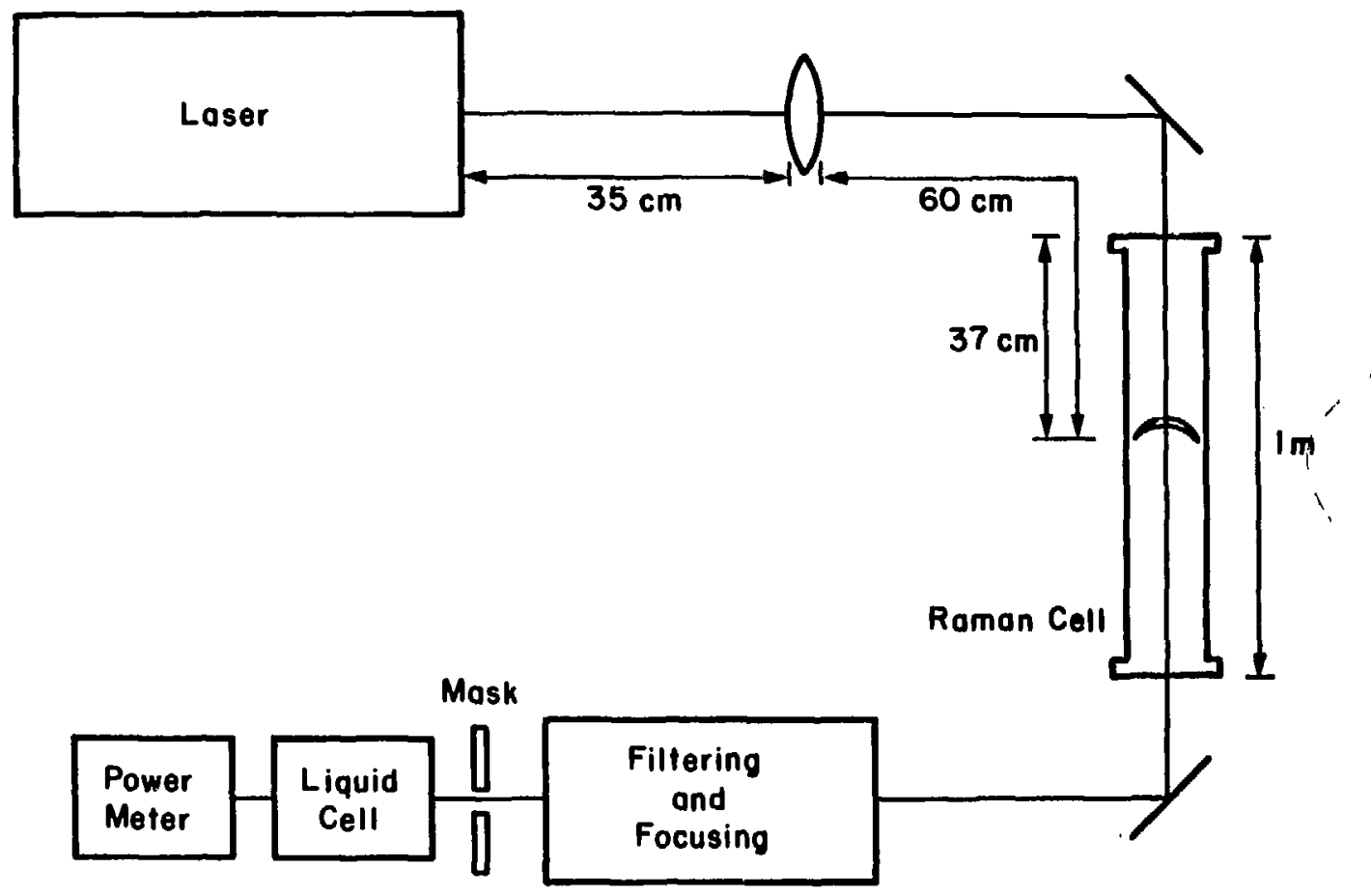

XBL $8212-12404$ 
fluence of $0.16 \mathrm{~J} / \mathrm{cm}^{2}$ and energy of $2.4 \mathrm{~mJ}$. The bean profile is not a uniform Gaussian because the final dye anplifier is longtitudinally pumped with the "doughnut" mode Quanta Ray.

For some experiments at $1.06 \mathrm{um}$, a Raytheon SS404 $\mathrm{Nd}^{+3}$ : YAG laser is used 9 . The maximum flux of $55 \mathrm{~mW} \mathrm{~cm}^{-2}$ at $1.06 \mathrm{um}$ was used in guantum yield measurements in $\mathrm{D}_{2} \mathrm{O}$. The pulse length is $15 \mathrm{~ns}$, the fluence $0.83 \mathrm{~J} / \mathrm{cm}^{2}$, and the bear area $1 \mathrm{~mm}^{2}$. The Raytheon beam profile is of good quality with no "doughnut". For $2.28 \mathrm{um}$ output, the laser pumps an angle tuned $\mathrm{LiNbO}_{3}$ optimal parametric oscillator 10 to generate a $1.2 \mathrm{~mJ}, 10 \mathrm{~ns}$ pulse with a $12 \mathrm{MW} \mathrm{cm}^{-2}$ flux at the conductivity cell. Pulse energies are measured with a Scientech 38-0101 calorimeter.

Comparisons of quantum yield measurements at $1.06 \mu \mathrm{m}$ show that the quantum yield is independent of electrode geometry, focussing parameters, and beam profiles. Table II-2 shows that measurements with three cells and three laser sources agree within 358 .

The cell is initially aligned with a visible beam by tilting the cell until the spot reflected from the rear window does not strike the electrodes. An Eastman Rodak IR Phosphor card is used to direct the near infrared light. Spurious signals result if the laser beam strikes the cell electrodes. The problem is eliminated by use of a mask. The mask is made of a pair of anodized 
Table II-1. Table of Raman cell output energies

\begin{tabular}{|c|c|c|c|c|c|c|}
\hline Dye & $\begin{array}{l}\text { Ranan } \\
\text { Mediun }\end{array}$ & & $\begin{array}{l}\text { Raman } \\
\text { Shift }\end{array}$ & $\begin{array}{l}\lambda \text { in } \\
(n n)\end{array}$ & $\begin{array}{l}\lambda \text { out } \\
\text { (nm) }\end{array}$ & $\begin{array}{c}\text { Energy } \\
(\mathrm{mJ})\end{array}$ \\
\hline DCM & $\mathrm{H}_{2}$ & 2nd & Stokes & 650 & 1410 & $>0.4$ \\
\hline Rh640 & $\mathrm{H}_{2}$ & 2nd & Stokes & 600 & 1197 & 1.6 \\
\hline Rh610 & $\mathrm{H}_{2}$ & 2nd & Stokes & 586 & 1142 & 2.25 \\
\hline $\operatorname{Rh} 610$ & $\mathrm{H}_{2}$ & 2nd & Stokes & 577 & 1110 & 2.4 \\
\hline Rh590 & $\mathrm{H}_{2}$ & 2nd & Stokes & 572 & 1090 & 1.4 \\
\hline Rh590 & $\mathrm{H}_{2}$ & 2nd & Stokes & 560 & 1047 & 2.1 \\
\hline $\mathrm{DCM}$ & $\mathrm{CH}_{4}$ & 2nd & Stokes & 628 & 990 & 1.7 \\
\hline DCM & $\mathrm{CH}_{4}$ & 2nd & Stokes & 624 & 980 & 1.3 \\
\hline $\mathrm{DCM}$ & $\mathrm{CH}_{4}$ & 2nd & Stokes & 615.8 & 961 & 0.75 \\
\hline RH6 40 & $\mathrm{CH}_{4}$ & 2nd & Stokes & 616.6 & 950 & 2.9 \\
\hline $\operatorname{Rh} 640$ & $\mathrm{CH}_{4}$ & 2nd & stokes & 605.8 & 937 & 1.2 \\
\hline Rh6 40 & $\mathrm{CH}_{4}$ & 2nd & Stokes & 600 & 923 & 3.5 \\
\hline DCM & $\mathrm{H}_{2}$ & $1 s t$ & Stokes & 663.8 & 916.5 & 2.0 \\
\hline DCM & $\mathrm{H}_{2}$ & lst & Stokes & 660 & 909 & 2.4 \\
\hline $\mathrm{DCM}$ & $\mathrm{H}_{2}$ & lst & Stokes & 650 & 890.5 & 2.7 \\
\hline $\mathrm{DCM}$ & $\mathrm{H}_{2}$ & lst & Stokes & 629 & 852 & 4.2 \\
\hline DCM & $\mathrm{H}_{2}$ & $1 s t$ & Stokes & 627 & 847 & 4.0 \\
\hline Rh6 40 & $\mathrm{H}_{2}$ & $1 s t$ & stokes & 611.6 & 820 & 4.0 \\
\hline RH640 & $\mathrm{H}_{2}$ & lst & Stokes & 600.4 & 800 & 3.5 \\
\hline RH590 & $\mathrm{H}_{2}$ & lst & Stokes & 564.7 & 738 & 5.1 \\
\hline
\end{tabular}

Optimum pressures for 2nd Stokes is 400-500 psi for both $\mathrm{H}_{2}$ and $\mathrm{CH}_{4}$. 
Optimum pressure for lst stokes output in hydrogen is $165 \mathrm{psi}$ at $738 \mathrm{~nm}$, increasing to $375 \mathrm{psi}$ at $916.5 \mathrm{~nm}$. Typical energy conversion efficiencies for lst stokes in hydrogen are between 10 and $17 \%$, 2nd stokes efficiency in hydrogen is $4-5 \%$. 
razor edges $1.4 \mathrm{mapart,} 9 \mathrm{~m}$ in front of the electrodes. The razor edges are oriented parallel to the electrode plane.

IV. Electronics

This section describes both the important and the unwanted features of the signal, then details the electronics chosen to record the important and eliminate the unwanted portions.

Important features of the conductivity versus time, Figure II-7, include (1) the pre-laser background conductivity, (2) the peak conductivity immediately following excitation, (3) the conductivity relaxation rate, and (4) the baseline increase after relaxation. unwanted features of the observed conductivity include (1) the small magnitude of the transient signal; especially when compared to the large background conductivity, and (2) the presence of large amplitude low frequency noise. The transient signal magnitude is as small as $3 \times 10^{-6}$ times the background conductivity resulting from equilibrium hydronium and hydroxide ion plus a small concentration of unidentified ions. The equilibrum conductivity has a time varying noise component with a frequency between D.C. and about $20 \mathrm{~Hz}$. The magnitude is up to 700 times as large as the signal magnitude. The noise comes from flow turbulence in the cell and from an undetermined 
Table II-2. Comparison of quantum yields for excitation at $9400 \mathrm{~cm}^{-1}$

\begin{tabular}{|c|c|c|c|}
\hline Cell & Laser Source & Temp. & $\varnothing$ \\
\hline Rnight et al. A & $\begin{array}{l}\mathrm{Nd}+3=9 \text { lass } \\
\text { Laser Associates }\end{array}$ & $\begin{array}{l}10 \\
25\end{array}$ & $\begin{array}{l}3.6 \times 10^{-7} \\
6.0 \times 10^{-7}\end{array}$ \\
\hline Expoxied Strip Quartz & Raman shifted dye & 10 & $2.8 \times 10^{-7}$ \\
\hline Teflon & Raman shifted dye & 10.6 & $3.25 \times 10^{-7}$ \\
\hline & $\begin{array}{l}\text { Raytheon } \\
\mathrm{Nd}^{+} 3: \text { YAG }\end{array}$ & $\begin{array}{l}10 \\
23 \\
25\end{array}$ & $\begin{array}{l}3.8 \times 10^{-7} \\
4.85 \times 10^{-7} \\
4.6 \times 10^{-7}\end{array}$ \\
\hline
\end{tabular}

A) B. Knight, D.M. Goodall, R.C. Greenhow, J. Chem. Soc. Faraday prans. 2, 75,841 (1979). 
source. Perhaps reactive metal atoms reduced from $\mathrm{Na}^{+}$ ions near the cathode catalytically produce $\mathrm{OH}^{-}$ions and $\mathrm{H}_{2}$ gas.

An A.C. coupled amplifier could have been used to eliminate problems caused by the two unwanted features of the signal by amplifying the small fast transient while filtering out the static background and low frequency noise. However, it would also produce baseline overshoot or baseline droop which distorts the magnitude of the baseline step.

The electronics actually used consists of four parts. The conductivity monitor separates the static conductivity from the transient conductivity and amplifies only the transient conductivity. The static conductivity is monitored separately. The triggered differential amplifier increases the transient voltage and attenuates the low frequency noise. The digitiz. records the signal. The optical isolator prevents laser noise from entering the system along triggering lines.

\section{A. Conductivity monitor}

The conductivity monitor, shown in Figure $I I-8$, is a conductivity bridge with transient eurrent differences between the two arms detected with an Analog Devices AD507K operated as a current to voltage converter. The 


\section{Figure II-7}

Transient conductivity in $\mathrm{H}_{2} \mathrm{O}$ showing (1) the pre-laser background conductivity, (2) the peak conductivity immediately following excitation, (3) the relaxation rate, and (4) the baseline increase after relaxation. The magnitude of the transient is as small as $3 \times 10^{-6}$ times the level of background conductivity, (1). The signal shown here has a peak magnitude about $10^{-4}$ times the background conductivity. The temperature is $19.8 \mathrm{C}$, with excitation at $9400 \mathrm{~cm}^{-1}$. 


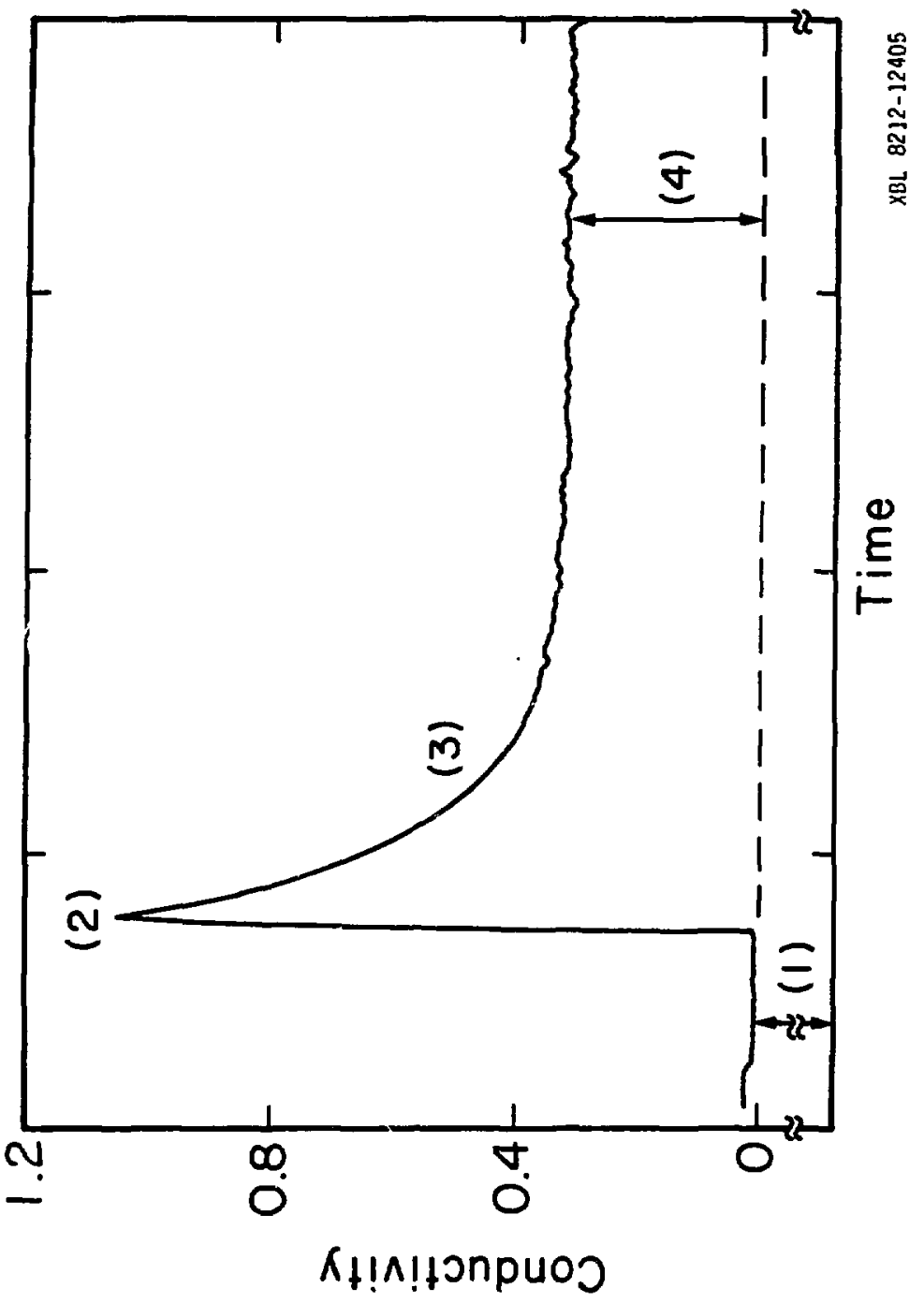


to ground by one arm of the conductivity bridge. The cell in the other arm is illuminated by the laser. Potentiometer R6 allows D.C. balance of the bridge to prevent current flow into the current to voltage converter in the absence of a transient conductivity. Bridge balance eliminates contribution from the static conductivity. Diodes $D 1$ and $D 2$ protect the amplifier input from voltage surges caused by switching the bias voltage polarity. Capacitors $C 2$ and $C 3$ prevent the bias voltage reference point from changing on the timescale of the transient conductivity signal. Capacitor $\mathrm{Cl}$ prevents the magnitude of the bias voltage from varying with time.

The voltage across $\mathrm{R} 3$ is monitored with voltage follower LM310 and is proportional to the equilibrum conductivity. Signal output from the current to voltage converter proceeds to the triggered differential amplifier.

B. Triggered Differential Amplifier

The triggered differential amplifier is shown in simplified form in Figure II-9. The circuit has two modes of operation depending on whether or not the signal is being digitized. When the conductivity is not being digitized, the FET is turned on, sending the same signal to both inputs of the CA3130 differential amplifier. This results in a gain of one and an oukput near zero volts. 
Eigure II-8

Schematic diagram of the transient and static conductivity monitor. Resistance is in ohms and capacitance in $\mu$ fd. $\mathrm{v}^{+}$and $\mathrm{V}^{-}$are $+15 \mathrm{v}$ and $-15 \mathrm{v}$. $\mathrm{RCI}$ and $\mathrm{RC} 2$ are the conductivity cell resistances. 


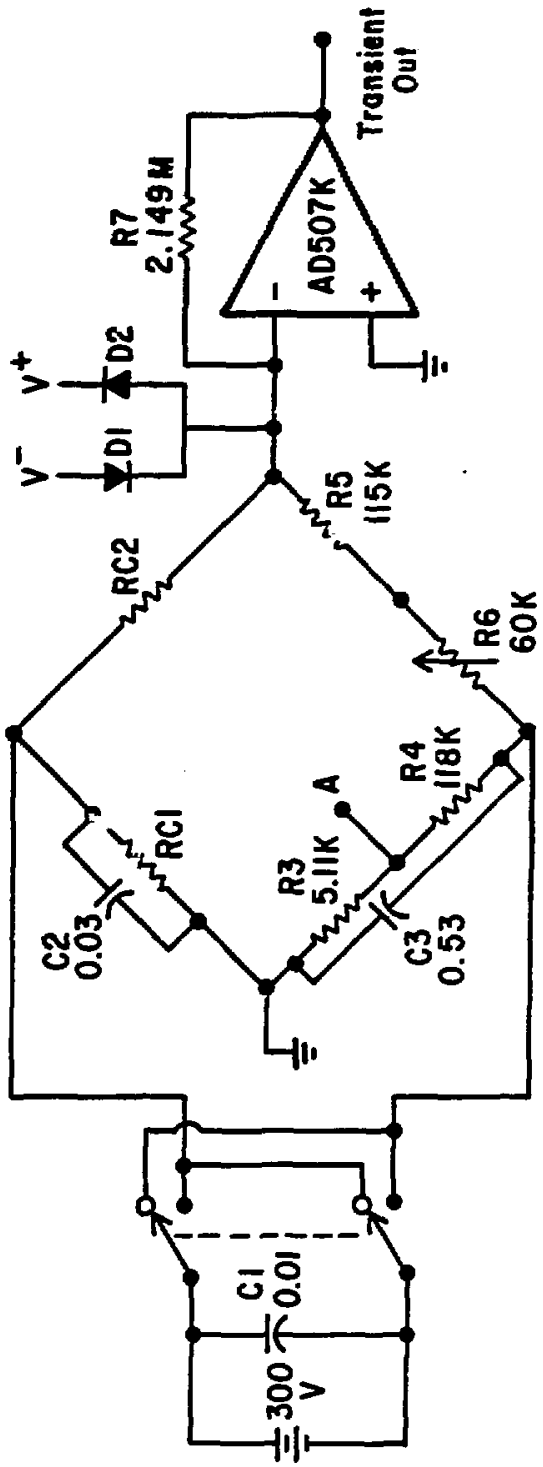

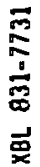

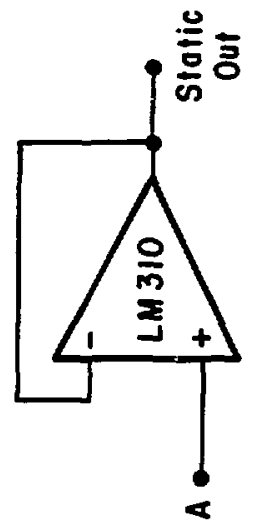


Figure II-9

Schematic diagram for the triggered differential amplifier. 


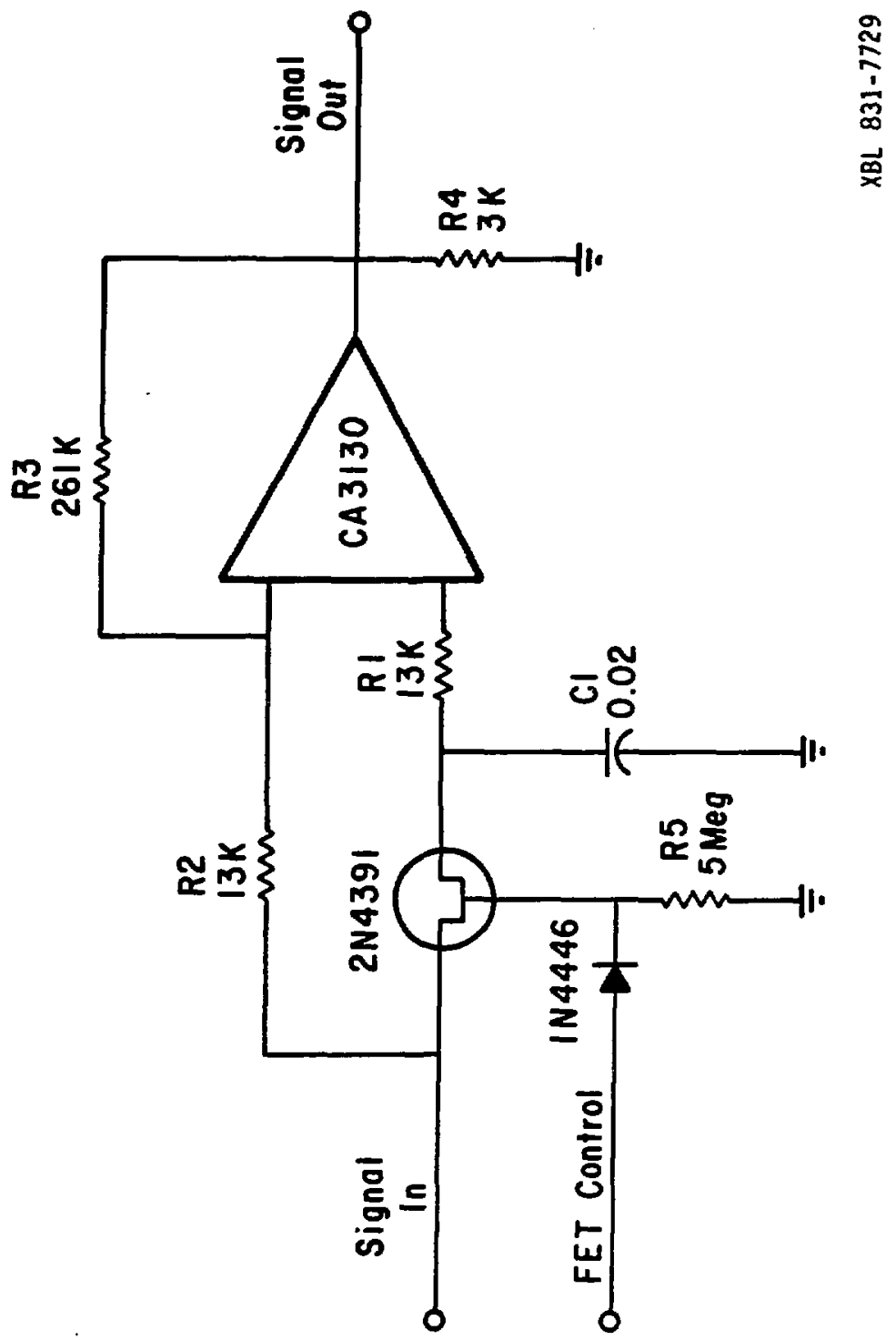


During this mode, low frequency noise is elininated. The mode of operation is changed after digitization begins. The FET is turned off, capacitor $\mathrm{Cl}$ stores the instantaneous signal voltage, and the circuit amplifies the difference between the stored voltage and the subsequent voltage with a gain of 20 . The system risetime from conductivity monitor input to triggered differential amplifier output is about 2.5 usec.

\section{Digitizer}

The signal is D.C. coupled to the 7 Al6 vertical amplifier of a Tektronix 7912 transient digitizer with a PDP $11 / 10$ Digital Equipment computer averager or to the 7Al6P vertical amplifier of a Tektronix 7912 AD transient digitizer with LSI-1l computer averager. The digitizers use an electron beam to write on a diode array, similar to the way an electron beam writes on the phosphor screen of an oscilloscope. Digitization occurs when a second electron beam reads the diode array. If high frequency noise is not filtered out, the writing beam intensity is spread over so many vertical diodes in some horizontal channels that no written signal is detected by the read beam. Inaccurate decay times and peak amplitudes can result.

A low pass filter preceding the digitizer input filters out high frequency noise. A cut off frequency 
of $300 \mathrm{kHz}$ is for signal decay times less than about $75 \mathrm{\mu s}$. The cut off frequency is $60 \mathrm{kHz}$ for longer decay times. The low pass filter also attentuates the signal for a net gain from triggered differential amplifier input to low pass filter output of 12.29 for $60 \mathrm{kHz}$ cut off and 6.3 for $300 \mathrm{kHz}$.

\section{Timing Electronics}

Three synchronized electrical pulses needed to control the experiment are shown in Figure II-10. They are (1) a digitizer trigger pulse, (2) a control pulse for the FET of the triggered differential amplifier, and (3) a laser Q-switch trigger needed to generate laser output. Generally, laser-generated electrical pulses are used for pulses (1) and (3). A pre-laser baseline is digitized during the time between pulse (1) and pulse (3). Occasionally, longer delays than provided by the laser are needed, A pulse generator and adjustable delay can be used to trigger the digitizer and laser. Pulse (2) is generated externally. This section describes the optical isolator used to isolate the laser electronics from the signal collection electronics and the timing circuit used to generate pulse (2). 
Figure II-10

Timing diagram of electrical pulses used for experimental control. Pulse (1) triggers a digitizer. Pulse (2) controls the triggered differential amplifier, and pulse (3) controls the laser Q-switch. (A) and (B) show the timing of the laser output and conductivity signal relative to the electrical pulses. 


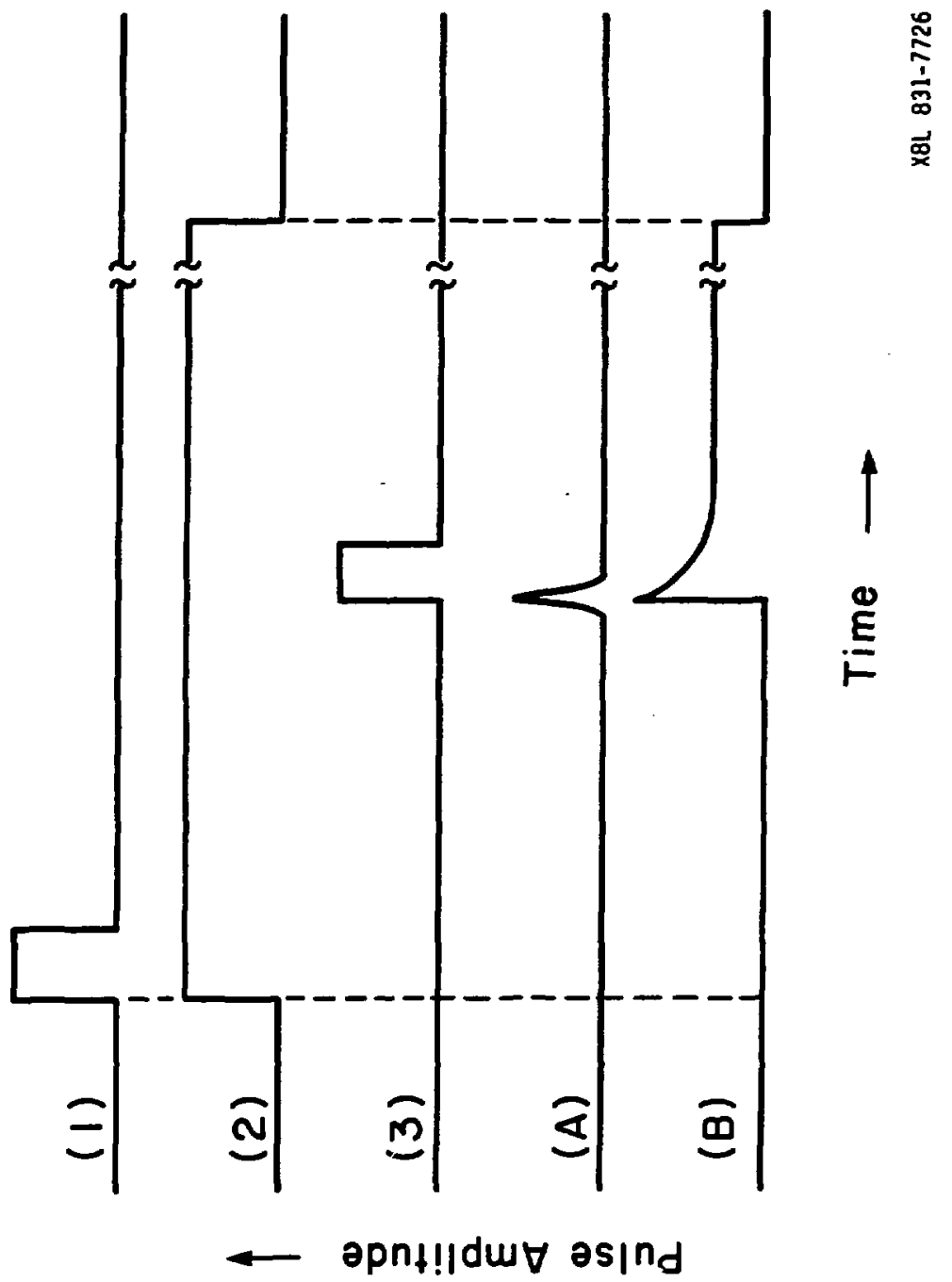


The schematic diagram in Figure II-1I shows an optical isolator which eliminates transmission of laser noise to the signal collection electronics. It consists of a 6 N136 optical isolator chip followed by an inverting amplifier capable of oriving both the signal averager and a timing circuit for the FET in the triggered differential amplifier. With the Quanta-Ray NA:YAG laser there is a delay of about 240 us between the lamp pulse out and the laser pulse. Digitizer triggering with the lamp out pulse, (1), allows a prelaser baseline to be recorded. With the Raytheon YAG laser there is an 200 us delay between the first amplifier out pulse (1) and laser output.

The timing circuit in Figure II-12 generates a gate which controls the FET. A pulse from the opto-isolator triggers the LM3li comparator, which starts a timing cycle in a 555 chip configured as a monostable multivibrator. The 7 or $60 \mathrm{~ms}$ output pulse turns off the FET after inversion by LM311, A2.

E. Electronics performance

Signal collection proceeds at the $10 \mathrm{~Hz}$ laser repetition rate. Typically, 512 shots are averaged, the conductivity cell polarity reversed, and the same number 
Figure II-II

Schematic diagram of optical isolator. Resistor values are from left to right, $300 \Omega$, I Megohm, $22 \mathrm{k} \Omega$, $49.9 \mathrm{k} \Omega, 200 \mathrm{k} \Omega$, and $51 \Omega$. The capacitor is $0.47 \mathrm{\mu Fd}$. 


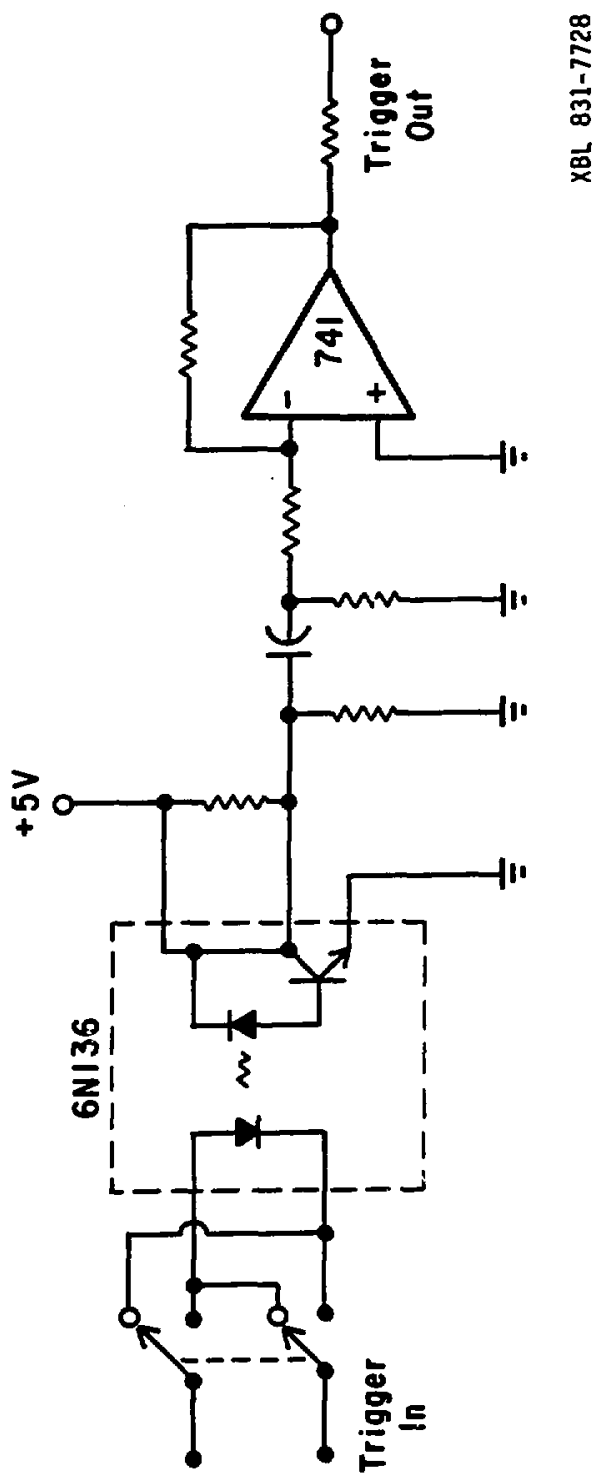


Figure II-12

Schematic diagram of timing circuit used to control the FET in the triggered differential amplifier. Resistor Rl is $62 \mathrm{~K}$ or $510 \mathrm{k} \Omega$ for a 7 or $60 \mathrm{~ms}$ output pulse. 


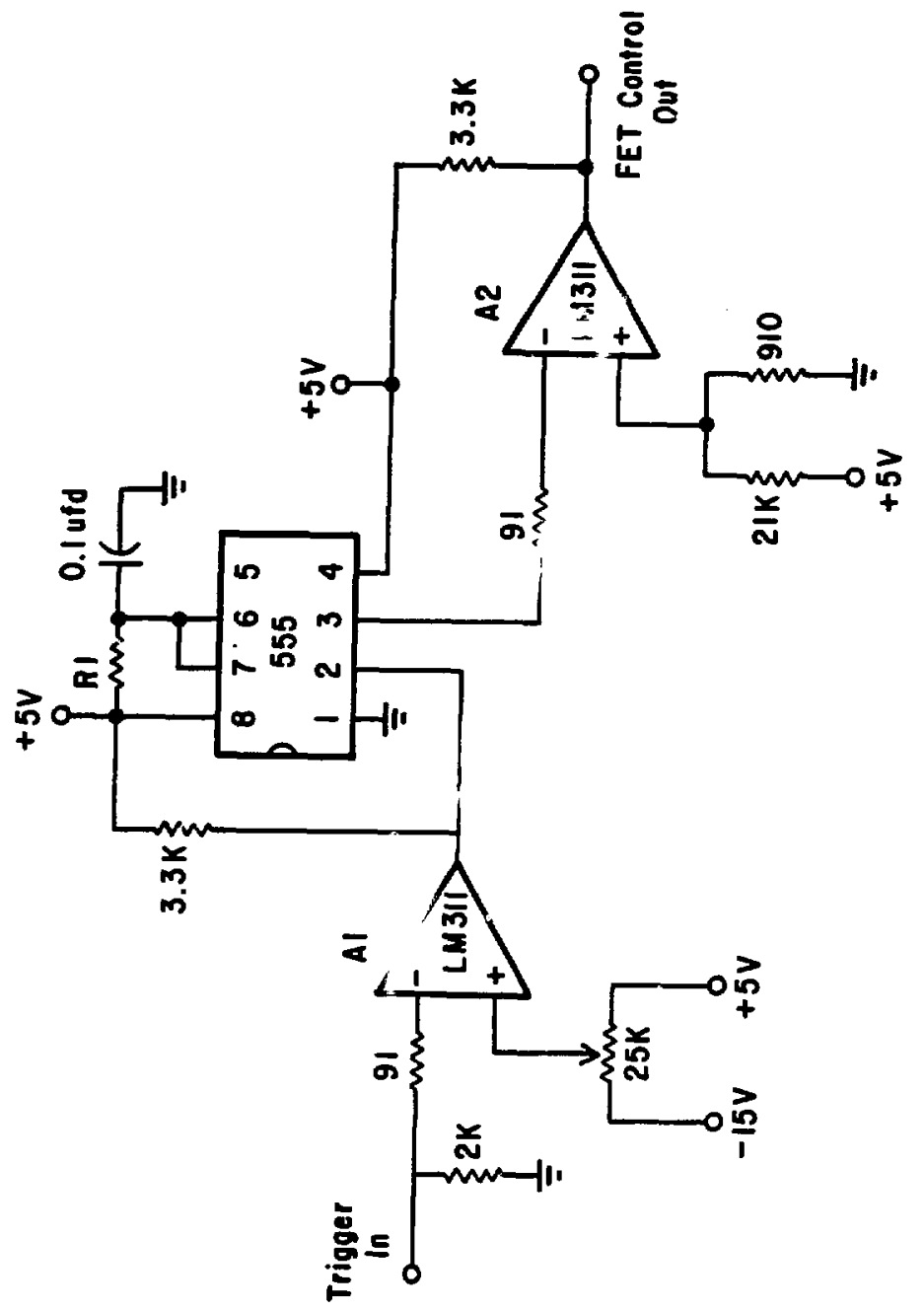

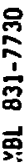


of additional shots averaged. Subtraction of the two signa_s eliminates pick-up and multiplies the conductivity signal by two. The subtracted pick-up is about $5 \times 10^{-10} \mathrm{~A}$ in magnitude. A step increase of about $1.5 \times 10^{-10}$ A with $300 \mathrm{~V}$ applied to the conductivity cell produces a conductivity step with signal to noise of one after averaging 1024 shots. This step increase corresponds to a $3 \times 10^{-13}$ molar change (3 ppm) in hydronium and hydroxide ion concentrations at $25^{\circ} \mathrm{C}$.

\section{v. Absorption spectra}

Absorption spectra between $1.19 \mathrm{\mu m}$ and $0.63 \mathrm{\mu m}$ were taken of $\mathrm{H}_{2} \mathrm{O} / \mathrm{D}_{2} \mathrm{O} \mathrm{mixtures}$ in a $10 \mathrm{~cm}$ cylindrical quartz cell using a Cary 14 spectrometer with an IRl detector. Mixtures were prepared by weight. Kesults at several wavel: igths of interest are shown in Table II-3. The spectra are shown in Figure II-13. The absolute baseline was set by comparison with $\mathrm{V}_{2} \mathrm{O}$ data by Sullivanll. the absorbance ir $\mathrm{H}_{2} \mathrm{O}$ at $650 \mathrm{~nm}$ is 138 greater than measured by Tam and Patel 12 by optoacoustic measurements. 
Figure $i r-13$

Absorption spectrum of :iquid $\mathrm{H}_{2} \mathrm{O}$ (A), 0.485 mole fraction hydrogen (B), 0.297 (C), 0.198 (D), 0.099 (E), $D_{2} \mathrm{O}$ (F), and the spectrometer baseline (G). Curves $D$ and $E$ should be shifted upward by 0.005 absorbance units. The pat: length is $10 \mathrm{~cm}$. 


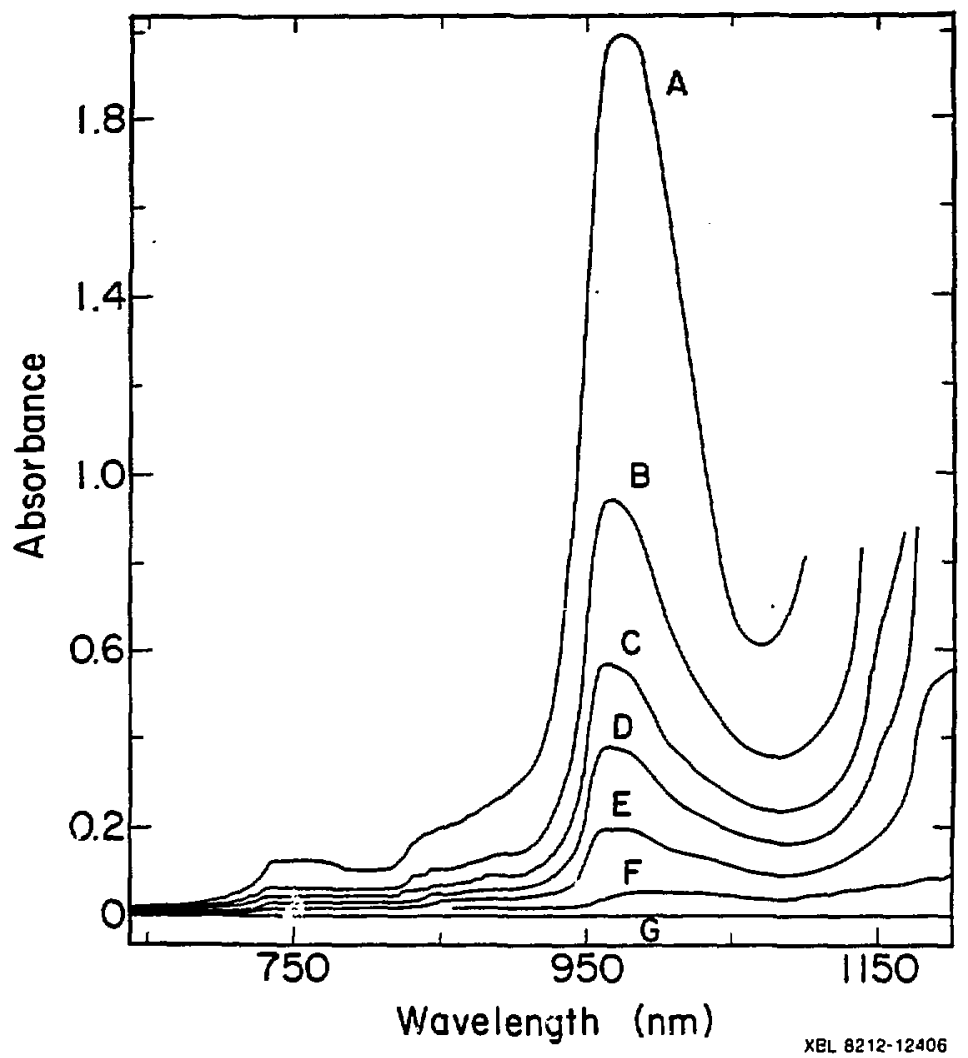


Table II-3. Absorbance ${ }^{a}$ of $\mathrm{H}_{2} \mathrm{O} / \mathrm{D}_{2} \mathrm{O}$ mixtures.

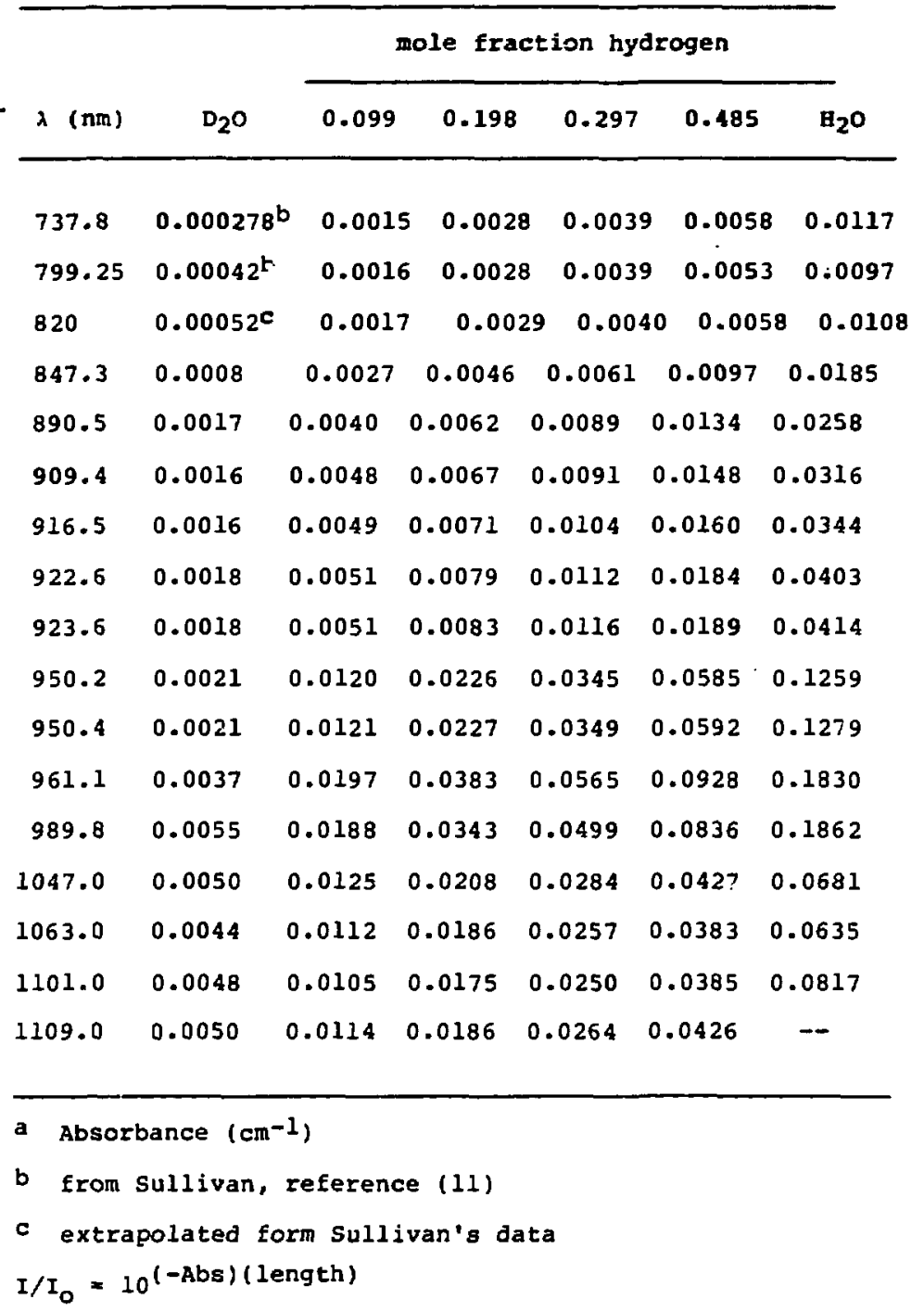


1. J.D. McIntyre and D.M. Kolb, Symp. Faraday Soc., 4, $99(1970)$.

2. A. Prostak, H.B. Mark, Jr., and W.N. Hansen, J. Phys. Chem., 72, 2576 (1968).

3. "Ultrapurity: Methods and Techniques" edited by Morris 2 ief and Robert Speights (Marcel Dekker, Inc., New York, 19721 .

4. G. Jones and B.C. Bradshaw, J. Am. Chem. Soc. 55, $1780(1933)$.

5. P.D. Goulden and P. Brooksbank, Anal. Chem. 47, 1943 (1975).

6. R. Byer and R.L. Herbst, in "Non-linear Infrared Generation", edited by Y.R. Shen (Springer-Verlag, New York, 1977).

7. A. Yariv, "Quantum Electronics" (J. Wiley and Sons, New York, 1975).

8. L. Young, Ph.D. Thesis, University of California, Berkeley (198I).

9. M. Zughul, Ph.D. Thesis, University of California, Berkeley (1978).

10. H.L. Dai, Ph.D. Thesis, University of California, Berkeley (1981).

11. S.A. Sullivan, J. Opt. Soc. Am. 53, 962 (1963).

12. A.C. Tam and C.K.N. Patel, Applied Optics, 18, 3348 (1979). 


\section{CHAPTER III. ANALYSIS}

The analysis consists of two parts. The first is a calculation of the quantum yield for the laser induced reaction. The second is a calculation of the thermal recombination rate from the equilibrium relaxation that follows perturbation by the laser-induced dissociative ionization. The end of the analysis section includes a discussion of experimental errors in the laser induced reaction.

I. Laser-induced dissociative ionization quantum yields

The goal of the data analysis is to relate the transient voltage changes shown in Figure II-7 to the chemical and physical processes of the dissociative ionization reaction. This section shows that the transient can be related most directly to reaction quantum yield. First, after some background, it is shown that the reaction kinetics can not be determined directly from the transient conductivity.

Absorption of a photon with energy greater than the dissociative ionization reaction threshhold initiates two competitive processes, both affecting the solution conductivity. The processes are (1) formation of separated product ions, $\mathrm{H}_{3} \mathrm{O}^{+}$and $\mathrm{OH}^{-}$, and (2) energy thermalization to produce a temperature jump. 
The kinetics of the two processes are described by the following equations:

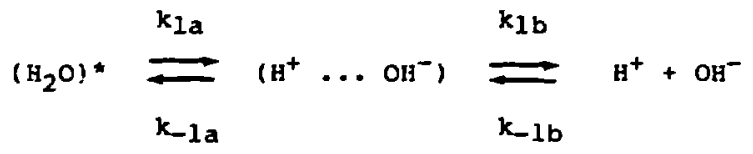

$$
\begin{aligned}
& \left(\mathrm{H}_{2} \mathrm{O}\right) * \underset{\mathrm{k}_{-2}}{=}\left(\mathrm{H}_{2} \mathrm{O}\right)
\end{aligned}
$$

$\left(\mathrm{H}_{2} \mathrm{O}\right)$ * denotes a vibrationally activated species, probably involving more than one $\mathrm{H}_{2} \mathrm{O},\left(\mathrm{H}_{2} \mathrm{O}\right)$ a vibrationally relaxed species, ( $\left.\mathrm{H}^{+} \ldots \mathrm{OH}^{-}\right)$an ion pair, and $\mathrm{H}^{+}+\mathrm{OH}^{-}$separated ions.

Processes (1) and (2) can be observed by monitoring the transient conductivity. The conductivity, $L$, is

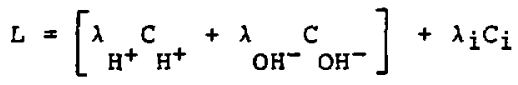

with $\lambda_{i}$ and $C_{i}$ the single ion equivalent conductivity and concentration of species $i$, respectively. So, process (1) directly increases the conductivity in proportion to the concentration of product ions $\mathrm{C}_{\mathrm{H}^{+}}$and $\mathrm{C}_{\mathrm{OH}^{-}}{ }^{-}$The temperature jump of process (2) increases the conductivity by increasing tne equivalent ionic conductivity, $\lambda$, of the pre-existing ions and increasing equilibrium ionic concentrations.

There are two timescales in the reaction kinetics that affect the conductivity. A rapid thermalization 
rate, $k_{2}$, and rapid ion pair formation rate, $k_{1 a}$, preceding a slow reiaxation of the $\mathrm{H}_{2} \mathrm{O} \rightleftharpoons \mathrm{H}^{+}$and $\mathrm{OH}^{+}$ disequilibrium, (3) in Figure II-7. If the electronics were infinitely fast, (1), the transient risetime in Figure II-7 would be related to $k_{1}$ and $k_{2}$. In practice, the electronics risetime is slow compared to $1 / k_{1}$ and $1 / k_{2}$, but fast compared to the equilibrium relaxation rate. This means neither $k_{1}$ nor $k_{2}$ can be determined from the signa risetime.

However, the quantum yield can be determined from step (4) in Figure II-7, and the equilibrium relaxation, (3), extrapolated to (2), the conductivity peak. The quantum, yield, $\phi$, for the production of separated ions is the fraction of vibrationally activated species that react.

$$
\phi=\frac{k_{1}\left[\left(\mathrm{H}_{2} \mathrm{O}\right)^{\star}\right]}{\left(\mathrm{k}_{1}+\mathrm{k}_{2}\right)\left[\left(\mathrm{H}_{2} \mathrm{O}\right)^{\star}\right]}
$$

where $k_{1}$ is the overall rate for production of separated ions. Recognizing

$$
\frac{k_{1}}{k_{1}+k_{2}}\left[\left(\mathrm{H}_{2} \mathrm{O}\right)^{*}\right]=\text { [reactive events] }
$$

and

$$
\left[\left(\mathrm{H}_{2} \mathrm{O}\right) *\right]=[\text { absorbed photons }]
$$




$$
\phi=\frac{\text { [reactive events] }}{\text { [absorbed photons] }}=\frac{\text { [product } \left.\mathrm{B}^{+}\right]}{\text {[absorbed photons] }}
$$

The remainder of this section shows how the guantun yield is calculated. The concentration of absorbed photons is calculated, from either the baseline step. which is proportional to the temperature jump, or from the absorption spectrum and transmitted laser energy. The concentration of reactive events is related to the conductivity jump. Also, the measured voltage changes must be related to the absolute conductivity changes.

\section{A. Concentration of Absorbed Photons}

The concentration of absorbed photons is calculated from either the measured conductivity step or from the absorption spectrum of the water. First, the method using the conductivity step is described.

1. Concentration of absorbed photons from the conductivity steps.

The concentration of absorbed photons is related to the temperature increase calculated from the conductivity step. The necessary equations for any composition and temperature are given. Calculated concentrations assume a uniform spatial distribution of absorbed photons and 
product ions. Actually, the laser intensity is not uniform and does not completely fill the interelectrode region. The actual laser geometry does not influence the calculated quantum yields as shown in Appendix A. The concentration of absorbed photons, [N], is given by

$$
[N]=\frac{C_{p \rho L T}}{h e v N_{A}}
$$

where $C_{p}$ is the specific heat, $p$ is the density, $N_{A}$ is Avogadro's number, and hev is the photon energy. For $\mathrm{H}_{2} \mathrm{O}$, equation $(8)$ becomes

$$
[N]=\frac{(28730) \Delta T}{V}
$$

where $v$ is in $\mathrm{cm}^{-1}, \Delta \mathrm{T}$ is in degrees Celsius and (N) is in moles $/ \mathrm{cm}^{3}$.

For $\mathrm{D}_{2} \mathrm{O}$

$$
[N]=\frac{(25780) \Delta T}{V}
$$

and for isotopic mixtures, a linear interpolation is made. $C_{p}$ and $p$ are considered temperature independent over the temperature range studied with values taken at $25^{\circ} \mathrm{C}$. 
For $\mathrm{H}_{2} \mathrm{O}$.

$$
\begin{aligned}
& c_{p}=0.998 \mathrm{cal} / \mathrm{gm}^{1} \text { and } \\
& p=0.99704 \mathrm{gm} / \mathrm{cm}^{3} 2 .
\end{aligned}
$$

For $\mathrm{D}_{2} \mathrm{O}$,

$$
\begin{aligned}
& c_{p}=1.004 \mathrm{cal} / \mathrm{gm}^{3} \text { and } \\
& p=1.10445 \mathrm{~g} / \mathrm{cm}^{3} 2 .
\end{aligned}
$$

For small conductivity changes, temperature changes are given by the derivative of equation (3).

$$
\Delta \mathrm{L}=\frac{\mathrm{dL}}{\mathrm{dT}} \Delta \mathrm{T},
$$

with the derivative given by

$$
\frac{d L}{d T}=\sum_{i}\left(\frac{d \lambda_{i}}{d T} c_{i}+\lambda_{i} \frac{d c_{i}}{d T}\right)
$$

Thus, two sources contribute to the conductivity step. The first term is the temperature-induced change in ionic mobilities and the second is the temperature-dependent water ionization equilibrium.

The conductivity and concentration of four different ions, $\mathrm{H}^{+}, \mathrm{OH}^{-}, \mathrm{Na}^{+}$, and $\mathrm{Cl}^{-}$, are used in equation (12). The theoretical concentration and conductivity of $\mathrm{H}^{+}$and $\mathrm{OH}^{-}$ions is used in equation (12). The concentration of $\mathrm{Na}^{+}$and $\mathrm{Cl}^{-}$is found by attributing the measured excess conductivity above that from $\mathrm{H}^{+}$and $\mathrm{OH}^{-}$to $\mathrm{Na}^{+}$ and $\mathrm{Cl}^{-}$. No signi cant error is introduced if the 
excess conductivity is produced by a fully dissociated species other than NaCl. (The discussion of errors section shows that the quantum yield is relatively insensitive to errors in the contribution of impurity ions to the conductivity step and jump.) The temperature dependence of the equivalent conductivity is given by the temperature dependence of the solvent viscosity independent of the ionic identity for small concentrations and temperature-independent ionic radii.

$$
\lambda_{i}=\frac{P_{i}}{n(T)}
$$

where $\eta(T)$ is the solution viscosity and $P_{i}$ is the proportionality constant. From Stokes' Law, $\mathrm{P}_{i}=$ $z e_{o} F / 1800 \pi r_{i} \cdot z e_{o}$ is the ionic charge, and $r_{i}$ the ionic radius. 4 The equivalent conductivities used for NaCl are as follows.

For $\mathrm{H}_{2} \mathrm{O}$

$$
\lambda_{\mathrm{Na}^{+}}+\lambda_{\mathrm{Cl}^{-}}=67.188+2.135(\mathrm{~T})+9.49 \times 10^{-3}(\mathrm{~T})^{2}
$$

with $T$ in degrees Celsius and $\lambda$ in $\mathrm{cm}^{2} \mathrm{ohm}^{-1} \mathrm{~mol}^{-1} .8$

For $\mathrm{D}_{2} \mathrm{O}$

$$
\lambda_{\mathrm{Na}^{+}}+\lambda_{\mathrm{Cl}^{-}}=\frac{\lambda_{\mathrm{Na}}+\left(25^{\circ} \mathrm{C}\right)}{i(\mathrm{~T})}
$$

where 


$$
\eta(T)=\exp [(669.2 /(T-133.98))-3.966]
$$

with $T$ in Kelvin and $n$ in centipoise. Equation (16) is obtained by modifying a functional form that works well for $\mathrm{H}_{2} \circ 5$ to fit data for $\mathrm{D}_{2} \circ{ }^{6}$ within about 2 from $20^{\circ} \mathrm{C}$ to $40^{\circ} \mathrm{C}$. The conductivity of $\mathrm{NaCl}$ in $\mathrm{D}_{2} \mathrm{O}, \lambda\left(25^{\circ} \mathrm{C}\right)$, is $104.2 \mathrm{~cm}^{2} \mathrm{ohm}^{-1} \mathrm{~mol}^{-1}$ after a small 0.28 correction to the data of Giere at $98.98 \mathrm{D}_{2} \mathrm{O} .{ }^{7}$ For mixtures of $\mathrm{H}_{2} \mathrm{O}$ and $\mathrm{D}_{2} \mathrm{O}$, a linear interpolation is made between equations (14) and (15). The error is 1.68 when compared to Giere's experimental result at $25^{\circ} \mathrm{C}$ in $47.88 \mathrm{D}_{2} \mathrm{O} .{ }^{7}$ Ionic conductivities used for $\mathrm{H}^{+}$and $\mathrm{OH}^{-}$ions are as follows:

For $\mathrm{H}_{2} \mathrm{O}^{8}$,

$$
\lambda_{\mathrm{OH}^{-}}=110.88+3.468(\mathrm{~T})
$$

with $\mathrm{T}$ in degrees Celsius and $\lambda_{\mathrm{OH}^{-}}$in $\mathrm{cm}^{2} \mathrm{ohm}^{-1} \mathrm{~mol}^{-1}$ fits experimental data9-11 within 68 from $0^{\circ} \mathrm{C}$ to $75^{\circ} \mathrm{C}$ and within 28 from $15^{\circ} \mathrm{C}$ to $75^{\circ} \mathrm{C}$.

$$
\lambda_{\mathrm{H}^{+}}=224.33+5.305(\mathrm{~T})-0.0113(\mathrm{~T})^{2}
$$

Fits experimental data9,11-13 within 28 from $-6^{\circ} \mathrm{C}$ to $55^{\circ} \mathrm{C}$. For $\mathrm{D}_{2} \mathrm{O}$ and $\mathrm{H}_{2} \mathrm{O} / \mathrm{D}_{2} \mathrm{O}$ mixtures at $25^{\circ} \mathrm{C}$, a linear extrapolation with mole fraction is unsatisfactory. However,

$$
\lambda_{\mathrm{L}^{+}}+\lambda_{\mathrm{OL}^{-}}=548.9 \mathrm{x}_{\mathrm{H}_{2} \mathrm{O}}+411.2 \mathrm{x}_{\mathrm{HDO}}+363.4 \mathrm{x}_{\mathrm{D}_{2} \mathrm{O}}
$$


is satisfactory with an error of 0.652 at 0.096 mole fraction deuterium?. Agreement with experimental data is within about 48 over the temperature range from $18^{\circ} \mathrm{C}$ to $75^{\circ} \mathrm{C}^{7}$ if the conductivity of an arbitrary mixture of hydrogen and deuterium is scaled by the temperature dependence of $\mathrm{H}_{2} \mathrm{O}$ conductivity given in equations (17) and (18). Equation (19) requires $\mathrm{H}_{2} \mathrm{O}, \mathrm{D}_{2} \mathrm{O}$, and $\mathrm{KDO}$ mole fractions of a given isotopic mixture. If the distribution of hydrogen in hydrogen/deuterium mixtures is statistical, then the equilibrium expression for

$$
\mathrm{H}_{2} \mathrm{O}+\mathrm{D}_{2} \mathrm{O} \rightleftharpoons 2 \mathrm{HDO}
$$

is

$$
[\mathrm{HDO}]^{2} /\left[\mathrm{H}_{2} \mathrm{O}\right]\left[\mathrm{D}_{2} \mathrm{O}\right]=4 .
$$

This approximate expression is used only to obtain a fit of hydrogen and hydroxide ion conductivities. The reacer should see reference (14) if a more precise expression for the equilibrium constant is required for other applications. Equation (12) also needs the temperature dependence of the equilibrium hydrogen and hydroxide ion concentrations, or $\mathrm{K}_{\mathrm{w}}(\mathrm{T})$. For $\mathrm{H}_{2} \mathrm{O}$,

$$
\mathrm{pK}=-6.118+4476.6 / \mathrm{T}+0.017105(T)
$$

with $T$ in Relvin, agrees within about 0.38 with experimental datals for $k_{w}$ between $0^{\circ} \mathrm{C}$ and $60^{\circ} \mathrm{C}$ and is in reasonable 
agreement with Ackermann's $s^{16}$ value at $80^{\circ} \mathrm{C}$. For $\mathrm{D}_{2} \mathrm{O}$,

$$
\mathrm{pK}=4900 /(\mathrm{T})-7.5527+0.0200854(\mathrm{~T})
$$

with the temperature in Kelvin, matches experimental results 17 for $K_{w}$ within about 0.78 between $5^{\circ} \mathrm{C}$ and $50^{\circ} \mathrm{C}$. The $\mathrm{pK}$ of $(22)$ is multiplied by a factor of 0.999 to agree with Gold and Lowe 18 at $25^{\circ} \mathrm{C}$. Then $K_{w}$ is calculated from the expression

$$
K_{w}=K_{H}[1-n+n \&]^{3}\left[1-n+n\left(K_{D} / K_{H}\right) 2^{-3}\right]
$$

where $\mathrm{K}_{\mathrm{H}}$ is $\mathrm{K}_{\mathrm{W}}$ for $\mathrm{H}_{2} \mathrm{O}, \mathrm{K}_{\mathrm{D}}$ is $\mathrm{K}_{\mathrm{W}}$ for $\mathrm{D}_{2} \mathrm{O}, \mathrm{n}$ is the deuterium mole fraction and 2 , taken to be 0.69 , is the fractionation factor for $\mathrm{L}_{3} \mathrm{O}^{+}$ions. I represents an $H$ or a $D$. Calculated values match experimental values at $25^{\circ} \mathrm{C}$ within 4.58 , with the maximum deviation at 0.5 mole fraction deuterium. Severdl alternate expressions for $\mathrm{K}_{\mathrm{W}}$ which $\mathrm{fit}$ the experimental data equally well are given by Gold and Lowel8. Finally, [N], the concentration of absorbed photons, is found by insertion of equations (14), (15), (17), and (23), their temperature derivatives, and the measured conductivit? step into equation (12) to give $\Delta T$. Then $\Delta T$ is inserted into equation (8). 


\section{Concentration of Absorbed photors from Absorption spectra.}

The method described above is not universal. In $\mathrm{D}_{2} \mathrm{O}$, and sone isotopic mixtures, the absorbance and the temperature dependence of $\mathrm{K}_{\mathrm{W}}$ are smaller than for $\mathrm{B}_{2} \mathrm{O}$. Therefore, the baseline step is too small to measure accurately. Instead, absorption spectra and transmitted laser energies are used to calculate a baseline step and the concentration of absorbed photons. The absorption spectrum and transmitted energy gives the energy deposited in the sulution. The baseline conductivity step is proportional to the deposited energy. The proportionality constant is given by calibration at wavelengths and isotopic compositions where a baseline step is readily observed.

The energy absorbed is given by

$$
P E_{0}-P E=\left(10^{+}=C 2-1\right) P E
$$

where $\mathrm{PE}_{\mathrm{O}}$ is the laser pulse energy before the cell, PE is e transmitted pulse energy, and ecl is the abscrbance of the water. Generally, the absorbance is calculited from Cary 14 spectra of isotopic mixtures given in the Experimental section. For wavelengths longer than $700 \mathrm{~nm}$, a linear interpolation is madr between the two spectra closest in hydrogen mole fraction to the 
sample being measured. For a few wavelengths shorter than $600 \mathrm{~nm}$, Cary 14 measurements at $650 \mathrm{~nm}$ as a function of hydrogen mole fraction are scaled to match results by Tam and Patel for $\mathrm{H}_{2} \mathrm{O}$ and $\mathrm{D}_{2} \mathrm{O}^{19}$. The equation used is

$$
\begin{aligned}
\varepsilon\left(\lambda, \mathrm{X}_{\mathrm{H}}\right)= & \frac{\varepsilon\left(650, \mathrm{X}_{\mathrm{H}}\right)-\varepsilon\left(650, \mathrm{D}_{2} \mathrm{O}\right)}{\varepsilon\left(650, \mathrm{H}_{2} \mathrm{O}\right)-\varepsilon\left(650, \mathrm{D}_{2} \mathrm{O}\right)}\left[\varepsilon_{\mathrm{TP}}\left(\lambda, \mathrm{H}_{2} \mathrm{O}\right)-\varepsilon\left(\lambda, \mathrm{D}_{2} \mathrm{O}\right)\right]+ \\
& \varepsilon_{\mathrm{TP}}\left(\lambda, \mathrm{D}_{2} \mathrm{O}\right)
\end{aligned}
$$

where $\varepsilon\left(\lambda, X_{B}\right)$ is the molar extinction coefficient at wavelength $\lambda$, and mole fraction hydrogen, $x_{H}$ - Subscript TP denotes data by Tam and Patel. Two sources are used for the pure $D_{2} O$ spectrum at wavelengths longer than $700 \mathrm{~nm}$. Cary 14 spectra are used for wavelengths longer than $820 \mathrm{~nm}$. Data by Sullivan 20 are used for wavelengths between $820 \mathrm{~nm}$ and $700 \mathrm{~nm}$.

The deposited energy, if measured in millijoules, is related to a conductivity step as follows:

$$
\left.\Delta T=\frac{\left(P E_{O}-P E\right)\left(2.39 \times 10^{-4}\right.}{\rho C_{P} V} \mathrm{cal} / \mathrm{mJ}\right)
$$

where $\rho$ is the density in $g / \mathrm{cm}^{3}, c_{p}$ is the heat capacity in cal $/ \mathrm{g}^{\circ} \mathrm{C}$, and $\mathrm{V}$ is the effective volume. Replacing $\Delta \mathrm{T}$ in equation (6)

$$
\Delta L=\frac{d L}{d T} \frac{\left(P E_{O}-P E\right)\left(2.39 \times 10^{-4}\right)}{\rho C_{p} V}
$$


where $\Delta \mathrm{L}$ is the confuctivity change, and $d L / d T$ is given in equations (11) and (12). All variables in this equation are known except $v$, the effective volume. To determine $v$, a wavelength and isotopic composition are hosen where both an absorbed energy and a conductivity ste. are easily measured. The values are inserted in equation (26) and it is solved for $v$. values at 1.06 um with hydrogen mole fractions of $1.0,0.9,0.40$, and $0.32 ; 0.847$ un with mole fractions 1.0 and 0.9 ; and $1.1 \mu \mathrm{m}$ with a hydrogen mole fraction of 0.32 were averaged to yield an effectize volume of $0.141 \pm 0.01 \mathrm{~cm}^{3}$. Once $v$ is determined, equation (26) is used to calculate baseline conductivity steps at other wavelengths and isotopic compositions. The method for determining the concentration of absorbed photons from the conductivity step was already described.

B. Concentration of reactive events

The remaining information needed to calculate the quantum yield is the concentration of reactive events. The concentration of reactive events is equal to the concentration of product hydronium ion. This shows how the concentration of product hydronium ion, $\Delta \mathrm{C}_{\mathrm{H}^{+}}$, is obtained from the peak conductivity. As mentioned previously, the two rapid processes influencing the peak conductivity are: (i) an increase in ionic mobility 
caused by the rapid temperature jump; and (ii) formation of ionic products. The contribution of process (ii) to the peak conductivity is found by subtracting the portion arrising from process (i). The contribution to the peak conductivity change from process (i) is proportional to the baseline conductivity step.

The conductivity magnitude is about one-third the step size. The lowest arrow curve $C$, Figure III-1 indicates the contribution to the peak from process (i). For small temperature and ionic concentration changes

$$
\begin{aligned}
& \Delta \mathrm{L}_{\text {peak }}=\Delta \mathrm{L}_{\text {mob }}+\Delta \mathrm{L}_{\mathrm{IP}} \\
& =\left[\left.\frac{\partial \mathrm{L}}{\partial \mathrm{T}}\right|_{\mathrm{C}_{\mathrm{i}}} \Delta \mathrm{T}\right]+\left[\left.\frac{\partial \mathrm{L}}{\partial \mathrm{C}_{\mathrm{H}^{+}}}\right|_{\mathrm{T}} \Delta \mathrm{C}_{\mathrm{H}^{+}}+\left.\frac{\partial \mathrm{L}}{\partial \mathrm{C}_{\mathrm{OH}^{-}}}\right|_{\mathrm{T}} \Delta \mathrm{C}_{\mathrm{OH}^{-}}\right]
\end{aligned}
$$

where $\Delta L_{m o b}$ represents the mobility induced conductivity change of process (i) and $\Delta \mathrm{L}_{\mathrm{IP}}$ is the ionic product induced conductivity change of process (ii). Equation (27) can be simplified by considering each term separately. First, consider the temperature-dependent mobility term. The temperature derivative of the conductivity is calculated in the previous section and is given in equations (11) and (12). The term in equation (12) involving $d c_{i} / d T$ is zero because there are no temperature induced changes in ionic concentrations immediately following the laser pulse; or 


$$
\Delta L_{\mathrm{mob}}=\sum_{i} c_{i} \frac{d \lambda_{i}}{d T} \Delta T
$$

Previously described equations for equilibrium ionic concentrations, $C_{i}$, ionic mobilities, $\lambda_{i}(T)$, and the temperature jump, $\Delta T$, are used to determine $\Delta \mathrm{L}_{\text {mob }}$. Now, consider the second term of equation (27). Replacing $\partial \mathrm{L} / \partial \mathrm{C}_{\mathrm{i}}$,

$$
\Delta \mathrm{L}_{\mathrm{IP}}=\lambda_{\mathrm{H}^{+}} \Delta \mathrm{C} \mathrm{H}^{+}+\lambda \mathrm{OH}^{-} \stackrel{\Delta \mathrm{C}}{\mathrm{OH}^{-}}
$$

since $\Delta \mathrm{C}_{\mathrm{H}^{+}}=\Delta \mathrm{OH}_{\mathrm{OH}^{-}}$

equation (29) becomes

$$
\Delta \mathrm{L}_{\mathrm{IP}}=\left(\lambda_{\mathrm{H}^{+}}^{+} \lambda_{\mathrm{OH}^{-}}\right) \Delta \mathrm{C}_{\mathrm{H}^{+}}
$$

Solution of equation (27) gives the concentration of product hydronium ion.

$$
\text { [reactive events] }=\Delta C_{\mathrm{H}^{+}}=\left(\Delta L-\Delta L_{\mathrm{mob}}\right) /\left(\lambda_{\mathrm{H}^{+}}+\lambda_{\mathrm{OH}^{-}}\right)
$$

Expressions for the ionic mobility of $\mathrm{H}^{+}$and $\mathrm{OH}^{-}$were given previously. Equation (8) and equation (30) are combined in equation (7) to give the quantum yield. 
c. Conductivity from signal voltages

The previous treatment uses solution conductivity changes in the data analysis. The transient signal voltages recorded during the experiment must be converted to solution conductivity changes. The conversion is described in this section as follows:

(1) The voltage change at the output of the conductivity monitor is calculated given a small change in conductivity.

(2) This voltage is amplified and the final voltage at the signal averager input is calculated, giving a relationship between cell conductivity changes and recorded voltages.

(3) The solution conductivity change is calculated given an identical small change in cell conductivity.

(4) The relationships are combined to yield the solution conductivity change as a function of recorded voltage change.

$\underline{(1)}$

The schematic for the cell amplifier is shown in Figure II-8. At bridge balance, the bias voltage across the illuminated cell, RC2, is

$$
v_{C}=\frac{R C 2}{R C 2+R 5+R 6} v
$$


where $\mathrm{V}$ is the $300 \mathrm{~V}$ bias battery. The current through the illuminated RC2 arm of the bridge is

$$
i_{c}=v_{c} / R C 2
$$

For small conductivity changes

$$
\Delta i_{c}=\frac{d i_{c}}{d(1 / R C 2)} \Delta(1 / R C 2)
$$

The derivative

$$
\frac{d i_{c}}{d(1 / R C 2)}=v_{c}-\frac{V R C 2(R 5+R 6)}{(R C 2+R 5+R 6)^{2}}
$$

The second term can be neglected for two reasons. First, RC2 is large compared to R5 + R6 so that the second term is only 3-48 of the first term. Also, $v_{c}$ is fixed by capacitors on the reference side of the bridge. The time constant for a change in $v_{c}$ is several tens of milliseconds, long compared to the transient signal. Thus,

$$
\Delta i_{c}=v_{c} \Delta(1 / R C 2)
$$

The $A D 507 K$ op arp forces the current differences between the two arms of the bridge through feedback resistor, R7. The voltage change at the conductivity monitor output is

$$
\Delta v_{C \pi}=-v_{C} R_{7} \Delta\left(1 / R_{C 2}\right)
$$


The cell monitor voltage is further amplified before being sent to the signal averager. The final voltage at the signal averager is

$$
\Delta v_{0}=\lfloor\text { Gain }\rceil \Delta v_{c m}
$$

where [Gain] is the gain of the triggered differential amplifier and low pass filter. Replacing $\Delta \mathrm{V}_{\mathrm{Cm}}$ and $\mathrm{V}_{\mathrm{C}}$ from equations (34) and (31)

$$
\Delta V_{0}=-\frac{[\text { Gain] } R C 2 V(R 7)}{(R C 2+R 5+R 6)} \Delta(1 / R C 2)
$$

(3) and (4)

The solution conductivity is

$$
L=\frac{k_{c 2}}{R C 2}
$$

where $k_{c 2}$ is the cell constant. For an identical small change in conductance $(1 / R C 2)$

$$
\Delta L=k_{c 2} \Delta(1 / R C 2)
$$

Rearranging (36) and $\Delta(1 / R C 2)$ in (38) gives an expression relating conductivity changes to measured voltage changes:

$$
\Delta L=\frac{-k_{c 2}(R C 2+R 5+R 6) \Delta V_{0}}{(R C 2)[\text { Gain] R7 V }}
$$


Numerical values are as follows: $k_{\mathrm{C}_{2}}=0.319 \mathrm{~cm}^{-1}$ : $\mathrm{RC2}=$ $5.27 \mathrm{M} \Omega$ at $25^{\circ} \mathrm{C}$ and 1108 of theoretical conductivity of pure water; $\mathrm{R} 5=115 \mathrm{~K} \Omega, \mathrm{R} 6=7 \mathrm{~K} \Omega, \mathrm{Gain}=12.29$ with a low pass filter setting of $60 \mathrm{KHz}$ and $\mathrm{V}=300 \mathrm{~V}$.

II. Thermal recombination rate

The exponential decay of the conductivity signal following equilibrium perturbation by the laser induced reaction is caused by recombination of the excess $\mathrm{B}^{+}$and $\mathrm{OH}^{-}$. The recombination rate, $\mathrm{k}_{-1}$ of equation $(2)$, is obtained from the experimental decay time, $\tau 21$.

$$
\frac{1}{\tau}=\mathrm{k}_{-1} \mathrm{IC}_{\mathrm{H}^{+}}+\mathrm{C}_{\mathrm{OH}^{-}} \mathrm{\jmath}+\mathrm{k}_{1}
$$

The ionic production rate, $k_{1}$, is ignored because it is small compared to the first term $(R)$. The equilibrium constant of equation (23) is used to calculate $\mathrm{C}_{\mathrm{H}^{+}}+\mathrm{C}_{\mathrm{OH}^{-}}$ when solving for $k_{-1}$.

\section{Discussion of Error}

This section describes the sources of both random and systematic error in the quantum yield determination. The error for determinations based on the conductivity jump and conductivity step are discussed first. Since the absorbance based quantum yields are calibrated against the conductivity based quantun yields, they will retain the systematic error of the conductivity based quantum 
yields. In addition, there is a calibration error and errors in the absorption spectrum not present in the conductivity based quantum yields.

\section{A. Conductivity Based Quantum Yields}

There are two sources of systematic error and two sources of random error. The systematic error comes from cell constant uncertainty and from errors in the expressions used for temperature-dependent ionic conductivities and the water equilibrium constant. The random error comes from statistical uncertainty in the peak conductivity and in the baseline step increase. The magnitude of each of these errors will be determined by estimating its contribution to the peak and step voltages used in the guantum yield calculation.

Error estimation requires knowing the sources of the conductivity signal. The lower arrow in curve $c$ of Figure III-1 indicates the contribution to the conductivity peak from the temperature-dependent mobility of $\mathrm{H}^{+}$and $\mathrm{OH}^{-}$ and of $\mathrm{Na}^{+}$and $\mathrm{Cl}^{-}$. In $\mathrm{H}_{2} \mathrm{O}$ near $25^{\circ} \mathrm{C}$ with a D.C. conductivity 108 greater than for ionically pure water, the arrow is located 0.31 times the baseline step. The remaining 0.69 of the baseline step comes from $\mathrm{H}^{+}$and $\mathrm{OH}^{-}$ equilibrium relaxation. of the $(0.31)$ (step) increase, 11.58 is from the temperature-dependence of the $\mathrm{Na}^{+}$and $\mathrm{Cl}^{-}$ionic conductivity and $\mathbf{8 8 . 5 8}$ is from the temperature 
Figure III-1

Transient conductivity signals at 16670 (A), 11740 (B), and $8440 \mathrm{~cm}^{-1}$ (C). The initial baseline for each signal is given by the dotted line $(\ldots)$. The conductivity jump Extrapolated to the time of the laser pulse is shown with an arrow $(+)$. The calculated jump for a quantum yield of zero is shown with an arrow $(+)$. 


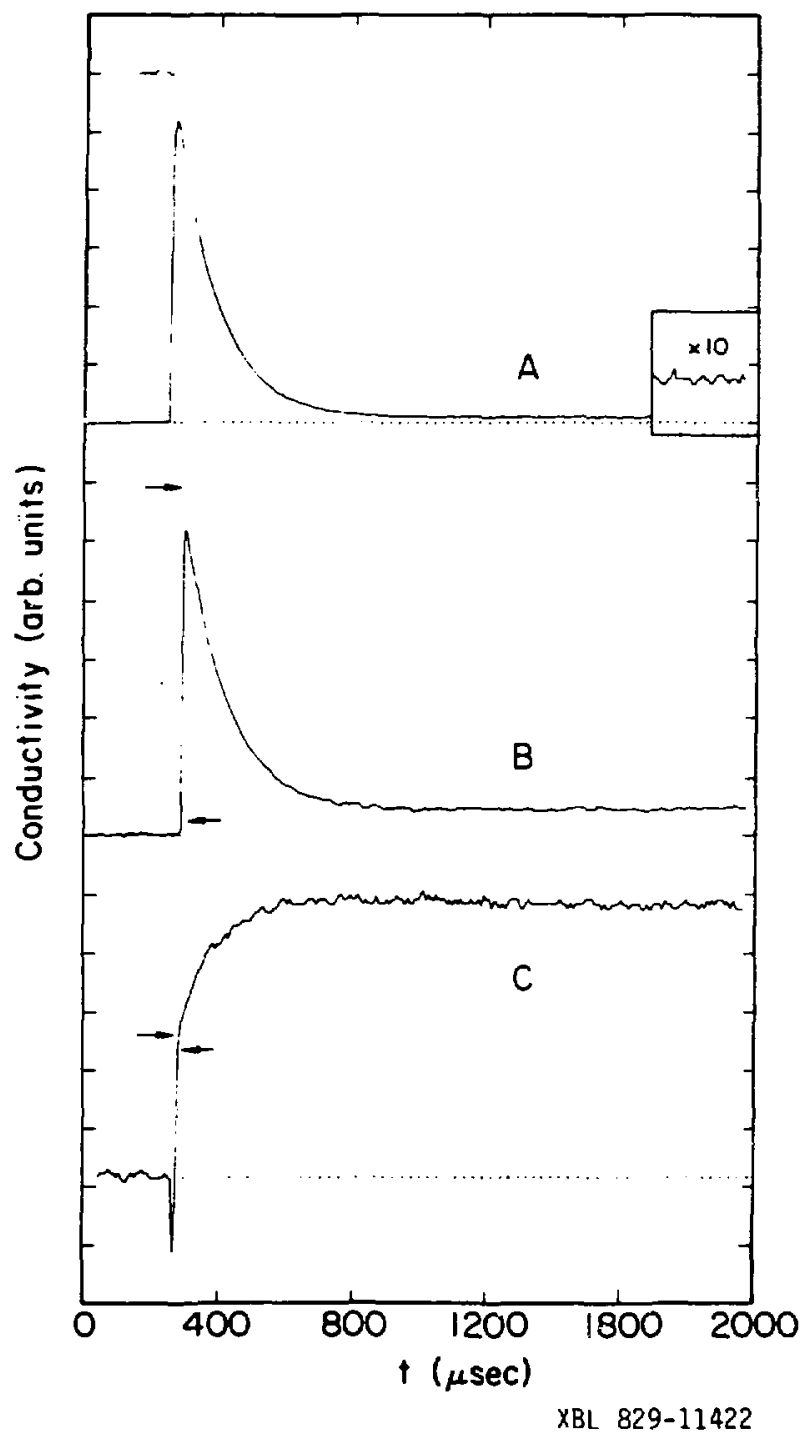


dependence of the $\mathrm{H}^{+}$and $\mathrm{OH}^{-}$ionic conductivity. These ratios hold with an accuracy of \pm 28 for $D_{2} \mathrm{O}$ as well. First, uncertainty in the posicion of the arrow is considered. The source of this uncertainty is uncertainty in the temperature derivative of the ionic conductivity. The maximum systematic error caused by uncertainty ir: the temperature derivative of ionic conductivities is estimated 22 to be 158 for $\mathrm{H}^{+}$and $\mathrm{OH}^{-} 23$ and 158 for $\mathrm{Na}^{+}$ and $\mathrm{Cl}^{-24}$. The uncertainty in the temperature derivative of $K_{W}$ is $<2 \%$. The total maximum e:ror in the calculated photon concentration is then

$$
(0.7)(0.02)+(0.3)(0.15)=68 .
$$

The total fractional error in the calculated contribution of the laser-induced ionization to the peak conductivity caused by an error in the position of the arrow is dependent on the relative magnitude of the peak and step. The approximate expression is

$$
\frac{(0.15)[(0.3) \text { step }]}{\text { peak }-[(0.3) \text { step }]}
$$

Thus the total error is excitation energy dependent. The maximum absolute error in the quantum yield is zero when the peak - to the step $\left(\phi=2 \times 10^{-7}\right.$ in $\left.\mathrm{H}_{2} \mathrm{O}\right)$, because the peak error and the step error cancel. The total error at $1.06 \mu$ is about 28 . When the colifluctivity signal looks like curve $A$ in Figure III-l, the error in the peak is insignificant and the baseline step error dominates, giving a total quantum yield error of 68 . 
The relative error for intermediate hydrogen mole fractions or as a function of temperature is the same. Another source of systematic error is uncertainty in the cell constant of the conductivity cell. An error in cell constant produces an error in the measured concentration of impurity ions and therefore an error in the calculated position of the arrow indicating the temperature-induced conductivity jump. Consider $\mathrm{H}_{2} \mathrm{O}$ near $25^{\circ} \mathrm{C}$ with a D.C. conductivity from impurities 108 greater than from $\mathrm{H}^{+}$and $\mathrm{OH}^{-}$. A 208 error in cell constant will triple the measured $\mathrm{Na}^{+}$and $\mathrm{Cl}^{-}$concentration and triple its contribution to the transient conductivity. It will change the position of the calculated arrow from 0.31 (step) to 0.35 (step), a less than 28 error in the peak at $1.06 \mu$ and a 78 error in the number of absorbed photons, for a net $5 \%$ quantum yield error. The wavelength dependence is identical to that already descrited. The relative error as a function of hydrogen mole fraction is nearly zero. The maximum combined systematic error from a 208 cell constant error and from ionic conductivity uncertainty is about 78 at $1.06 \mu$.

There are also two sources of random error. There are statistical baseline errors and statistical peak errors. The statistical peak errors are typically about 48 of the distance above the final baseline, independent of absolute peak height. The statistical baseline step uncertainty is about $1 \times 10^{-3} \mathrm{~V}$ for a low pass filter setting of $0.06 \mathrm{MHz}$, with 1280 shots averaged at each polarity. 
Table III-1

\begin{tabular}{lc} 
hydrogen mole fraction & statistical baseline uncertainty \\
\hline 0.00567 & 208 \\
0.0174 & 208 \\
0.038 & 158 \\
0.153 & 108 \\
0.3179 & 58 \\
1.0 & $<28$
\end{tabular}


Table III-1 shows the estimated statistical baseline step error for data at $1.06 \mathrm{~mm}$ as a function of hydrogen mole fraction. The statistical peak error exceeds the baseline error for $1.06 \mathrm{\mu m}$ excitation at a hydrogen mole fraction > 0.4.

B. Absorbance based quantum yields

Quantum yields based on the absorption spectra are not dependent on the baseline step increase. Thus, statistical baseline error is eliminated. Systematic and random uncertainty from the zero absorbance point of the spectrometer and pen reproducibility is addeó. After the zero absorbance point is set, measured absorbances at $650 \mathrm{~nm}$ differ from those of Tam and Patel 19 at $650 \mathrm{~nm}$ by about 0.00016. Their stated absolute accuracy is about 0.00014 . The pure $D_{2} O$ measurement differs by about $0.0005 \mathrm{~cm}^{-1}$. Therefore, an absolute accuracy of $0.0005 \mathrm{~cm}^{-1}$ is assumed. In addition, a statistical uncertainty of about $0.0005 \mathrm{~cm}^{-1}$ is found for the spectrometer pen position. Table III-2 shows the systematic and random error from uncertainty in the absorption spectrum at an excitation wavelength of $1.06 \mathrm{\mu m}$. Comparison of absorbance based and conductivity step based quantum yielas at $1.06 \mathrm{um}$ for hydrogen mole fractions between 0.15 and 0.32 shows agreement within about 108 . 
Table III-2

$$
\begin{aligned}
& \text { Quantum yield error from absorbance } \\
& \text { uncertainty }
\end{aligned}
$$

mole fraction hydrogen

$$
\text { statistical systematic }
$$

$\begin{array}{lcc}\mathrm{D}_{2} \mathrm{O} & 12.58 & 12.58 \\ 0.0174 & 98 & 98 \\ 0.038 & 78 & 78 \\ 0.1108 & 48 & 48 \\ 0.198 & 2.78 & 2.78\end{array}$




\section{Analysis references}

1. N.S. Osborne, B.E. Stimson, and D.C. Ginnings, J. Res. Natl. Bur. Std. 23, 197 (1939).

2. R.C. Weast, ed., "Handbook of Chemistry and Physics", 53rd edition, CRC Press, Cleveland (1972) p. F-4.

3. I. Rirshenbaum, "Physical Properties and Analysis of Heavy Water", McGraw-Hill, New York (1951).

4. J. O'M. Bockris, "Modern Electrochenistry", v.1, p 471 Plenun, New York (1970).

5. G.S. Kell in "Water: A Comprehensive Treatise". Vol. 1, edited by . Franks, Plenum Press, New York (1972) P. 407 .

6. R.C. Hardy and R.L. Cottingham, J. Chem. Phys., 17. 509 (1949).

7. V.A. Giere, Z. Naturforschung, 5a, 581 (1950).

8. David Goodall, private communication.

9. A.S. Quist and W.L. Marshall, J. Phys. Chem., 69, $2984(1965)$.

10. R.N. Marsh and R.H. Stokes, Aust. J. Chem, 17, $740(1964)$.

11. R.A. Robinson and R.H. Stokes, "Electrolyte Solutions", Butterworth (1959).

12. M. Nakahara, N. Takisawa, and J. Osugi, J. Phys. Chem., 85, $112(1981)$. 
13. B.B. Owen and F.H. Sweeton, J. Am. Chen. Soc., 63, 2811 (1941).

14. W.J. Albery in "Proton Transfer Reactions", edited by E.F. Caldin and v. Gold, Chapran and Ball, London (1975) p. 263.

15. H.S. Harned and R.A. Robinson, Trans. Faraday Soc., 36. $973(1940)$.

16. Th. Ackermann, 2. Elektrochem., 62, 111 (1958).

17. A.K. Covington, R.A. Robinson, and R.G. Bates, J. Phys. Chem., 70, 3820 (1966).

18.. v. Gold and B.M. Lowe, J. Chem. Soc. (A), 936 (1967).

19. A.C. Tam and C.R.N. Patel, Applied Optics, 18, 3348 (1979).

20. S.A. Sullivan, J. Opt. Soc. Am., 53r 962 (1963).

21. M. Eigen, พ. Kruse, G. Maass, and L. De Maeyer, Progr. Reaction Rinetics, 2, 285 (1964).

22. The error in the slope given a deviation from a curve $y(x)$ over a range in $x$ and $y$ of $R_{X}$ and $R_{Y}$, respectively, is taken to be $(4)$ (deviation of $y) / R_{y}$

23. Using footnote 22: $4(0.04)(650) / 700=158$

for a 48 deviation and a range of ionic mobilities of $700 \mathrm{~cm}^{2} \mathrm{ohm}^{-1}$ equivalent.

24. Using footnote 22: $4(0.02)(135) / 70=158$

for a 28 deviation and a range of ionic mobilities of $70 \mathrm{~cm}^{2} \mathrm{ohm}^{-1}$ equivalent. 


\section{CHAPTER IV. RESULTS}

Results are presented in two parts. The first part presents guantum $y$ :elds of the laser-induced reaction. The second part presents thermal recombination rates.

\section{Laser-induced Reaction}

Quantun yields for the laser-induced reaction are presented as a function of fluence, photon energy, temperature, and mole fraction hydrogen. The reader should keep in mind that as the mole fraction of hydrogen is increased from 0 to 1.0 , the composition of the solution changes approximately as in Equation ( 20 ) of the analysis section. Most hydrogen is in the form HOD at low hydrogen mole fractions. The initial sransient voltage as a function of laser pulse energy or fluence is shown in Figures $I V-I$ and $I V-2$. Figure IV-l shows results for $\mathrm{H}_{2} \mathrm{O}$ at $10^{\circ} \mathrm{C}$. The initial transient voltage is proportional to the quantum yield. The dependence is linear in pulse energy over a factor of twenty at $12,500 \mathrm{~cm}^{-1}$ and a factor of fifty at $16,670 \mathrm{~cm}^{-1}$. Figure IV-2 shows results for $0.12 \mathrm{X}_{\mathrm{H}}$, the mole fraction of hydrogen in $\mathrm{D}_{2} \mathrm{O}$. The initial peak height is linear in the pulse energy over a factor of twenty for excitation at $16500 \mathrm{~cm}^{-1}$. The absolute peak magnitude for excitation at $11802 \mathrm{~cm}^{-1}$ in 0.0057 mole fraction hydrogen in $D_{2} O$ is 60 to 70 times smaller than for pure $\mathrm{H}_{2} \mathrm{O}$. The reduction is caused by a lower overtone absorption $(x 1 / 20)$, lower 
Figure IV-1

The dependence of quantum yield on fluence in $\mathrm{B}_{2} \mathrm{O}$ near $10^{\circ} \mathrm{C}$. Normalized initial transient voltage $\left(\Delta \mathrm{V} / \Delta \mathrm{V}_{\mathrm{m}}\right)$ as a function of normalized pulse energy $\left(E / E_{m}\right)$; ( $\square$ ) data at $16670 \mathrm{~cm}^{-1}$ where $\Delta V_{\mathrm{m}}$ is $0.525 \pm 0.015 \mathrm{~V}$ and $E_{\mathrm{m}}$ is $11.5 \pm$ $0.1 \mathrm{~mJ} ;(\Delta)$ data at $12500 \mathrm{~cm}^{-1}$ where $\Delta V_{\mathrm{m}}$ is $0.144 \pm 0.008 \mathrm{~V}$ and $E_{m}$ is $2.02 \pm 0.05 \mathrm{~mJ}$. The solid lines represent the behavior expected for a single-photon process. $10 \mathrm{~mJ}$ corresponds to an intensity of $50 \pm 25 \mathrm{MW} \mathrm{cm}^{-2}$ and a fluence of $0.4 \pm 0.2 \mathrm{~J} \mathrm{~cm}^{-2}$. 


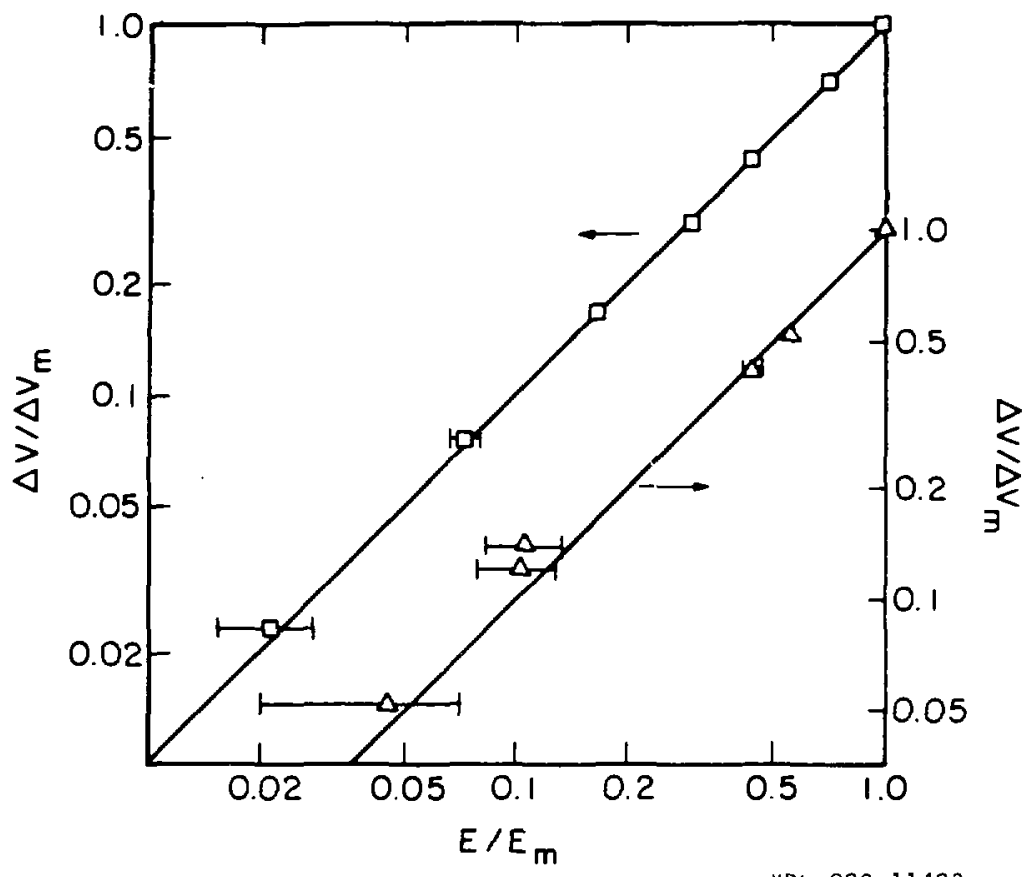

XBL 829-11423 
Figure IV-2

The dependence of the quantum yield on fluence is shown. The normalized initial transient voltage $\Delta V / \Delta V_{m}$ as a function of the normalized pulse energy $E / E_{m}$. (•) represents data for 0.12 mole fraction hydrogen in $\mathrm{D}_{2} \mathrm{O}$. The temperature is $24^{\circ} \mathrm{C}$. The photon energy is $16,500 \mathrm{~cm}^{-1}$. $\Delta V_{\mathrm{m}}$ is $0.023 \mathrm{~V}$ and $E_{\mathrm{m}}$ is $9.2 \mathrm{~mJ}$. 


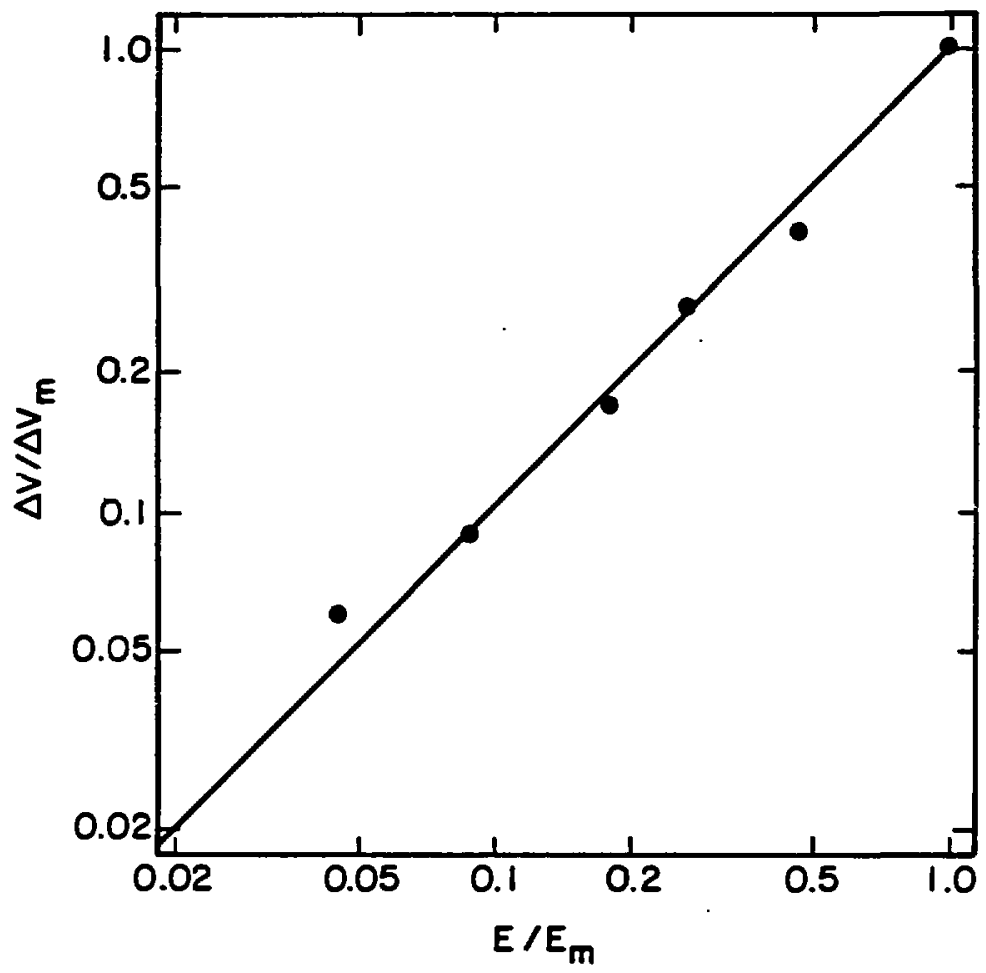

X61 831-7701 
product ion mobilities ( $x 1 / 1.5$ ), and a lower quantum yield $(x 1 / 2)$ in $D_{2} O$.

The photon energy dependence of the quantum yield between $8000 \mathrm{~cm}^{-1}$ and $17,000 \mathrm{~cm}^{-1}$ is shown for $\mathrm{H}_{2} \mathrm{O}$ and $0.12 \mathrm{x}_{\mathrm{H}}: 0.512 \mathrm{x}_{\mathrm{H}}, 0.406 \mathrm{x}_{\mathrm{H}}, 0.318 \mathrm{x}_{\mathrm{H}}$; and less than $0.01 .7 \mathrm{X}_{\mathrm{H}}$ in Figures $I^{\mathrm{V}-3}, \mathrm{IV}-4$, and IV-5. The absorption bands in this wavelength range correspond to $\mathrm{O}-\mathrm{H}$ or $\mathrm{O}-\mathrm{D}$ stretching overtones or to stretch plus bend combinations. For $\mathrm{H}_{2} \mathrm{O}$, the departure from a smooth increase of with $v$ is outside the limits of experimental error. The most striking feature of Figure IV-3 is that superimposed on the trend of increasing with increasing $v$ noted in reference 1 is a pattern which correlates with the absorption spectrum. The guantum yield rises rapidly on the low-frequency side of a band and is relatively constant through the band center and high-freguency wing.

No gualitative difference in behavior is seen between bands involving pure stretching excitation and those involving combination with bending vibrations. A small hump in the quantum yield at the $3 v_{s}+v_{b}$ overtone is present.

In the wavelength range shown, the absorption strength of a $\mathrm{D}_{2} \mathrm{O}$ molecule is more than an order of magnitude smaller than for an $\mathrm{H}_{2} \mathrm{O}$ or HOD molecule. This means that at 0.12 $\mathrm{X}_{\mathrm{K}}$, mole fraction of hydrogen added to $\mathrm{D}_{2} \mathrm{O}$, the absorption spectrum is roughly proportional to that of $\mathrm{H}_{2} \mathrm{O}$ as shown in 


\section{Figure IV-3}

Quantum yield $(\phi)$ as a function of photon energy $(v)$ for 0.12 mole fraction hydrogen in $D_{2} \mathrm{O}$ at $24 \pm 1^{\circ} \mathrm{C},(0)$. Quantum yield for photoionization of $\mathrm{H}_{2} \mathrm{O}$ at $10 \pm 1^{\circ} \mathrm{C}$, (0) as a function of photon energy (v). A line is drawn through experimental points. Estimated errors are shown for $\mathrm{H}_{2} \mathrm{O}$. For 0.12 mole fraction hydrogen, $\log (\phi)$ has a 108 random uncertainty at $9400 \mathrm{~cm}^{-1}, 158$ near $11100 \mathrm{~cm}^{-1}$, and 358 near $12200 \mathrm{~cm}^{-1}$. The absorption spectrum of $\mathrm{H}_{2} \mathrm{O}$ is shown as the dependence of the natural absorption coefficient $(a)$ on wavenumber $(v) .\left[I / I_{0}=\exp (a l).\right]$ Band assignments shown (ref. 2-4) with subscripts b and $s$ representing bending and stretching modes, respectively. 


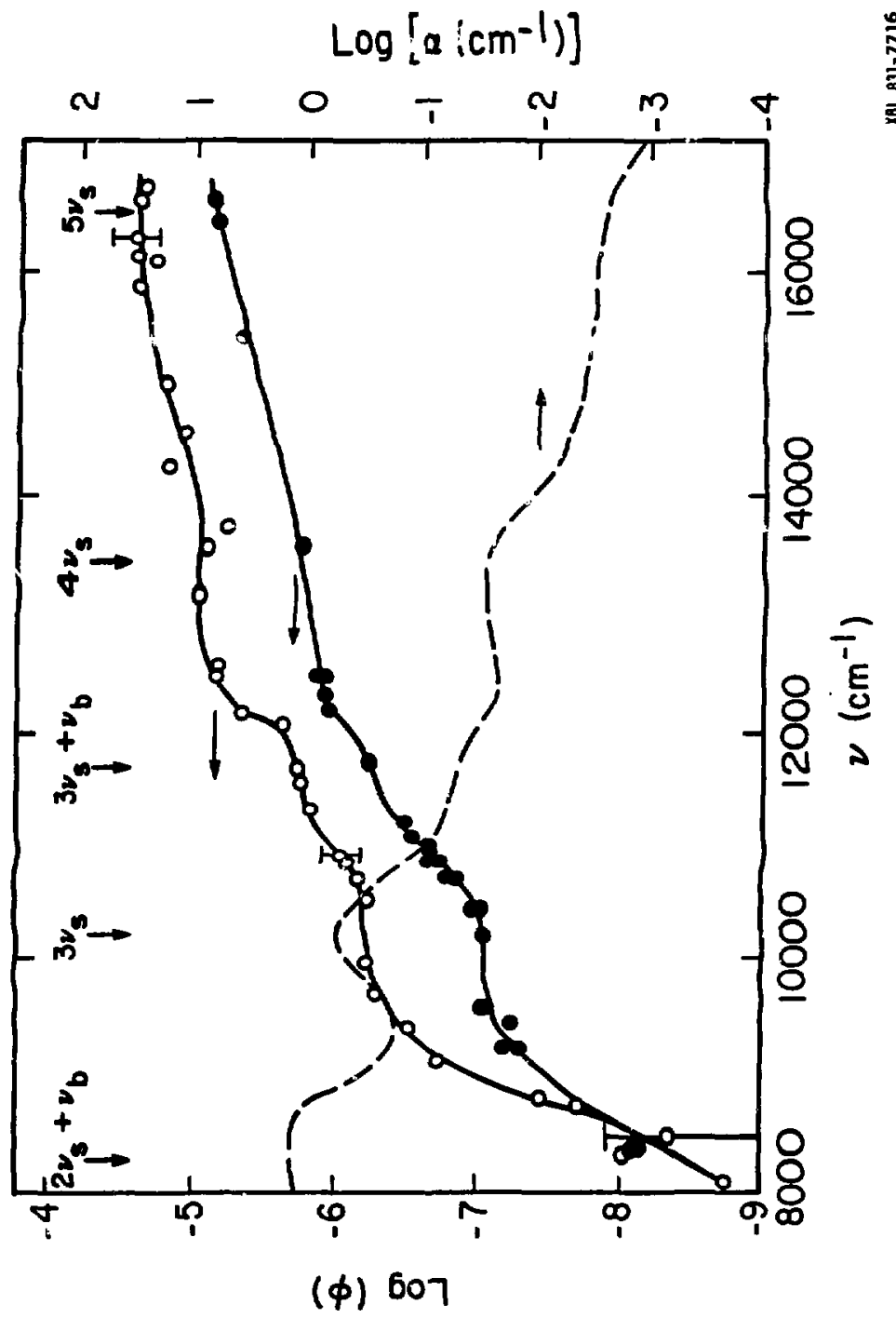


Figure IV-4

Quantum yield as a function of photon energy for photoionization of several mole fractions of hydrogen in $\mathrm{D}_{2} \mathrm{O}$. Experimental points for successive mole fractions are displaced upward by one unit. The bottom of the graph represents $\log _{10}(\phi)=-8,-9$, and -10 for $x_{H}=0.512(x)$, 0.406 (O), and $0.318(0)$, respectively. The vertical scale is +ldecade/division. The solid line has the same shape as the quantum yield for $\mathrm{H}_{2} \mathrm{O}$, but is displaced to overlay the experimental points. Random error varies from about 78 at $9400 \mathrm{~cm}^{-1}$ to 178 at $12200 \mathrm{~cm}^{-1}$. 


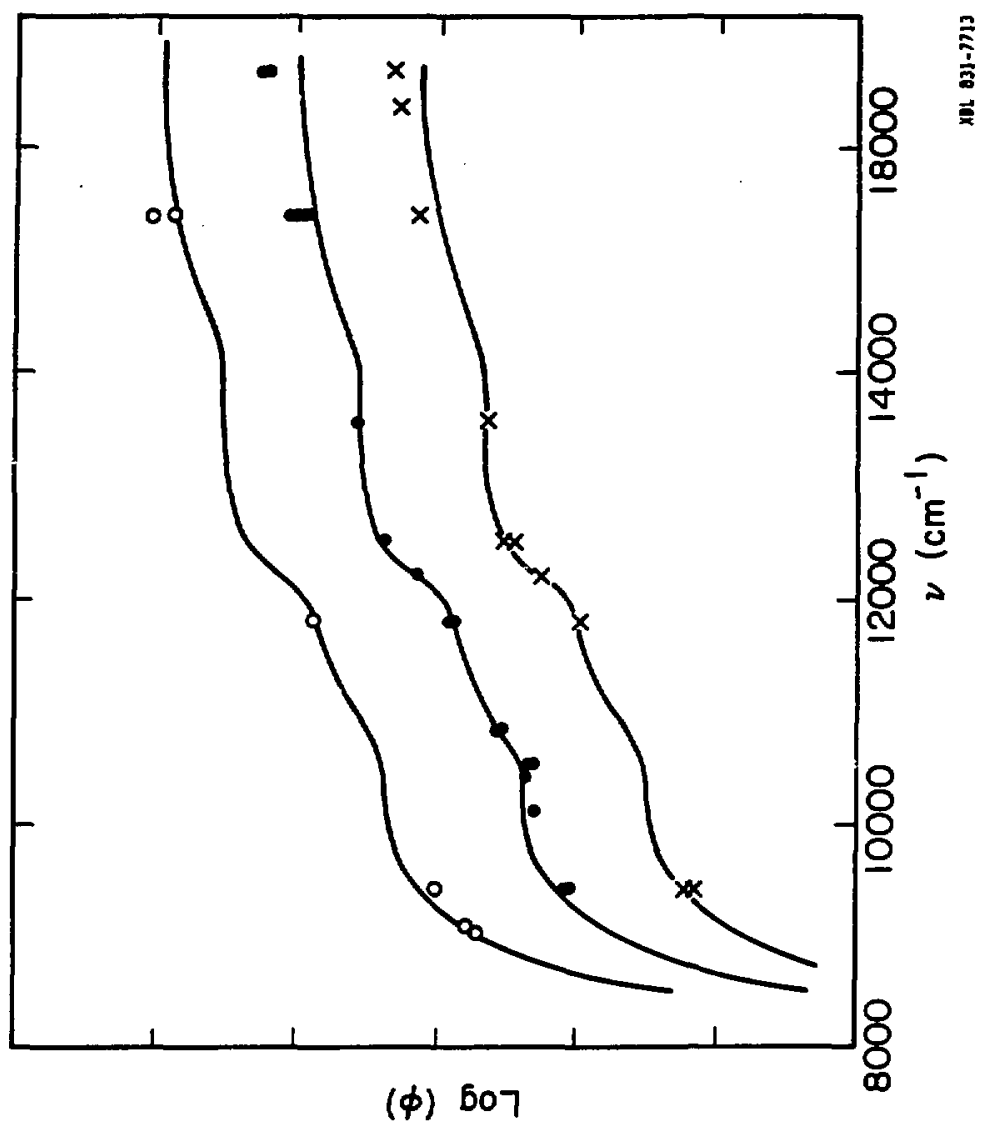


Figure IV-5

Quantum yield $(\phi)$ as a function of photon energy $(v)$ in predominantly $\mathrm{D}_{2} \mathrm{O}$. The mole fraction of hydrogen is $x_{H} \leq 0.017$. The relative error can be estimated from points. shown in the figure. 


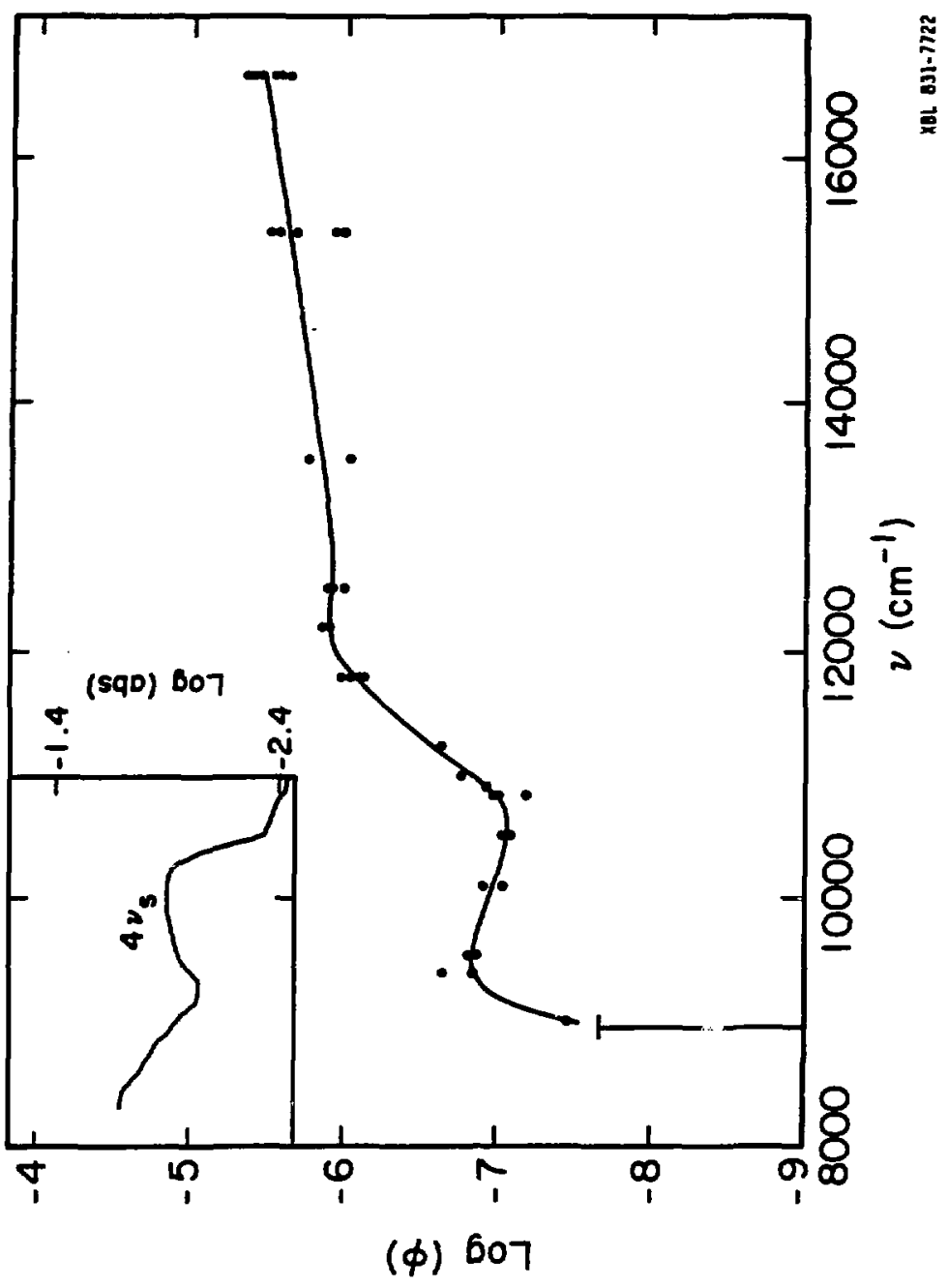


Figure II-13. The quantum yield of the $X_{H}=0.12$ mixture is shaped like the $\mathrm{H}_{2} \mathrm{O}$ quantum yield, but is displaced to lower yields. The quantum yields for higher hydrogen mole fractions, Figure IV-4, show behavior similar to $\mathrm{x}_{\mathrm{H}}=0.12$. Figure IV-5 shows the quantum yield along with the absorption spectrum near the $v=4, O-D$ stretch in nearly pure $\mathrm{D}_{2} \mathrm{O}, \mathrm{X}_{\mathrm{H}}<0.017$. At this composition, the absorption is mostly by $D_{2} O$ molecules. The quantum yield rises sharply on the red side of the $v=4,0-D$ stretch overtone absorption, then declines through the center of the band. It goes through a minimum on the blue side of the band, then rises as hirhur overtones begin contributing to the absorption. Additional structure in the quantum yield or the absorption spectrum from higher overtones is not resolved.

The first observable quantum yield is from excitation of the $\mathrm{v}=3 \mathrm{O}-\mathrm{H}$ stretch in $\mathrm{H}_{2} \mathrm{O}$ at $10250 \mathrm{~cm}^{-1}$ and the $\mathrm{v}=4$ $O-D$ stretch in $D_{2} O$ at $9000 \mathrm{~cm}^{-1}$. There is no known feature in the absorption spectrum of isotopic mixtures at energies higher than $8000 \mathrm{~cm}^{-1}$ enabling selective excitation of either HOD or $\mathrm{D}_{2} \mathrm{O}$. However, for dilute $\mathrm{H}_{2} \mathrm{O}$ in $\mathrm{D}_{2} \mathrm{O}$, a band centered at $7168 \mathrm{~cm}^{-1}$ is attributed to the overtone of the O-H stretch of HOD molecules.5.6 $\mathrm{D}_{2} \mathrm{O}$ absorption is small. Excitation of this feature at $7072 \mathrm{~cm}^{-1}$ in $0.038 \mathrm{x}_{\mathrm{H}}$ did not produce an observable laser-induced reaction.

Figure IV- 6 shows the temperature dependence of the quantum yield for five excitation wavelengths in $\mathrm{H}_{2} \mathrm{O}$. The 
Figure IV-6

$\mathrm{H}_{2} \mathrm{O}$ quantum yield as a function of temperature for excitation of four quanta of $0-B$ stretch: (A) $13570 \mathrm{~cm}^{-1}$. (x) $12484 \mathrm{~cm}^{-1}$; three quanta of stretch plus one bend, (0) 11737; or three quanta of stretch, (+) $10065 \mathrm{~cm}^{-1}$. (•) $9399 \mathrm{~cm}^{-1}$. The quantum yields at each wavelength are normalized by the quantum yield at $0^{\circ} \mathrm{C}, \phi(T=0)$. Random error is approximately 108 for excitation of $v=3$, and 158 for excitation of $v=4$. 


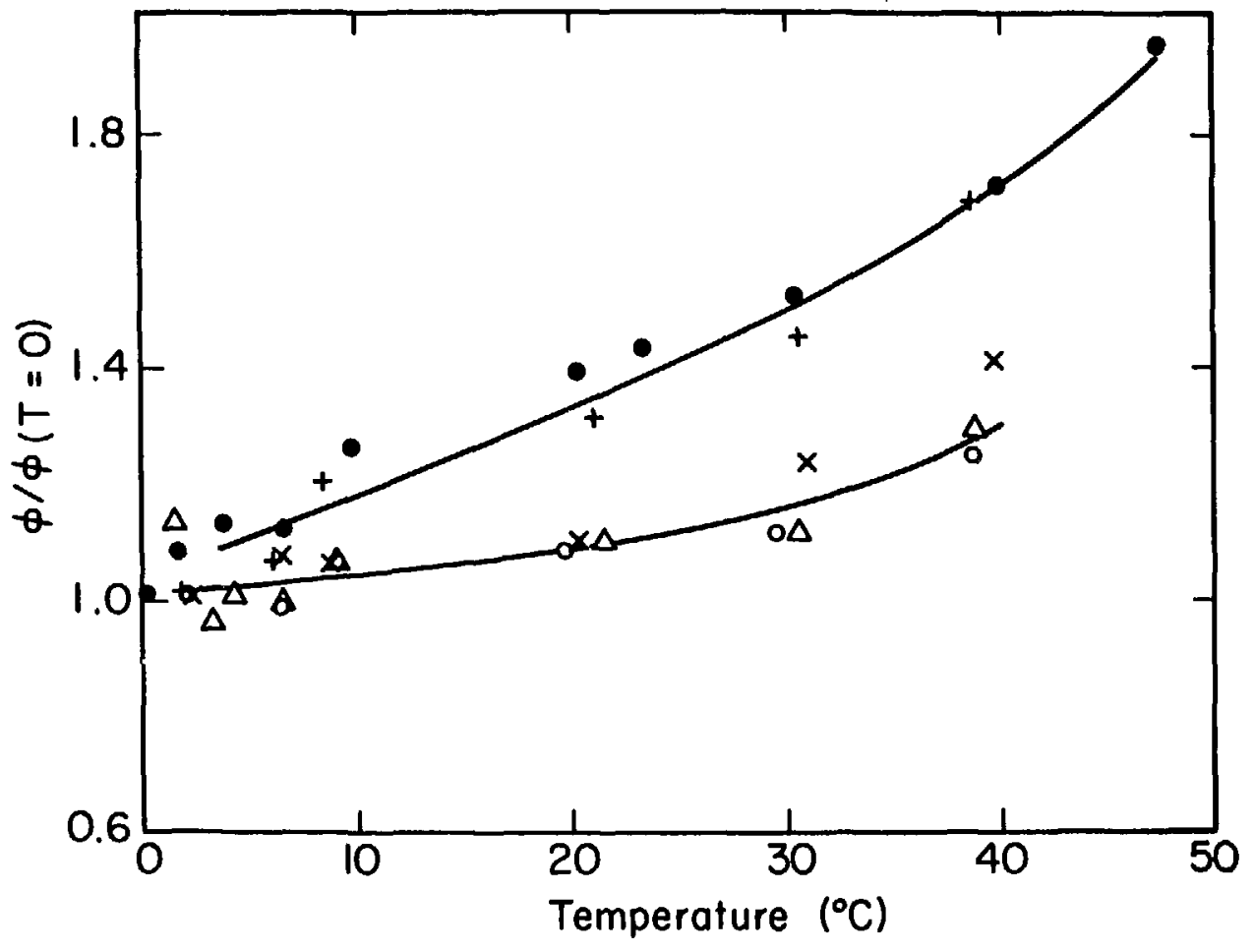

X8L 83$)-7719$ 
guantum yield increases as temperature increases. The fractional increase is smaller with a higher photon energy. As temperature goes from 0 to $10^{\circ} \mathrm{C}$, the quantum yield increase is a factor of 1.7 for three quanta of $0 H$ stretch and a factor of 1.3 for three quanta of stretch plus one bend or for four guanta of OH stretch. Figure IV-7 shows the temperature dependence of the guantum yield for nearly pure $\mathrm{D}_{2} \mathrm{O} ; \mathrm{X}_{\mathrm{H}}=0.017$, for excitation at $9400 \mathrm{~cm}^{-1}$. The yield increases a factor of 1.8 from $0^{\circ} \mathrm{C}$ to $40^{\circ} \mathrm{C}$, almost identical to the behavior of $\mathrm{H}_{2} \mathrm{O}$.

The structure of liquid water becomes more ordered as the temperature is reduced below $4^{\circ} \mathrm{C}^{7}$. Measurements at $9400 \mathrm{~cm}^{-1}$ show no change from the general decline of $\phi$ with decreasing temperature as the temperature is reduced below $4^{\circ} \mathrm{C}$.

The dependence of the quantum yield on hydrogen mole fraction is shown in Figure IV-8 for excitation at $11800 \mathrm{~cm}^{-1}$ and $9400 \mathrm{~cm}^{-1}$ and in Figure IV-9 for excitation at $15380 \mathrm{~cm}^{-1}$. Figure IV-8 shows a 2.5 times larger quantum yield for $\mathrm{H}_{2} \mathrm{O}$ than for $\mathrm{D}_{2} \mathrm{O}$. However, the increase in quantum yield is not monotonic with increasing hydrogen mole fraction. A quantum yield minimum occurs for about a 0.2 hydrogen mole fraction. Figure IV-IO shows 9400 wavenumber conductivity curves recorded at $0.0174,0.15,0.60,0.81$, and 1.0 mole fraction hydrogen. Figure IV-9 shows an eleven times larger quantum yield for $\mathrm{H}_{2} \mathrm{O}$ than for $\mathrm{D}_{2} \mathrm{O}$ at $15380 \mathrm{~cm}^{-1}$. The increase with greater hydrogen mole fraction is monotonic. 
Figure IV-7

The quantum yield of nearly pure $D_{2} \mathrm{O}, \mathrm{x}_{\mathrm{H}}=0.0174$, as a function of temperature for excitation of four quanta of O-D stretch at $9400 \mathrm{~cm}^{-1}$. The quantum yield is normalized by the quantum yield at $0^{\circ} \mathrm{C}, \phi(T=0)$. 


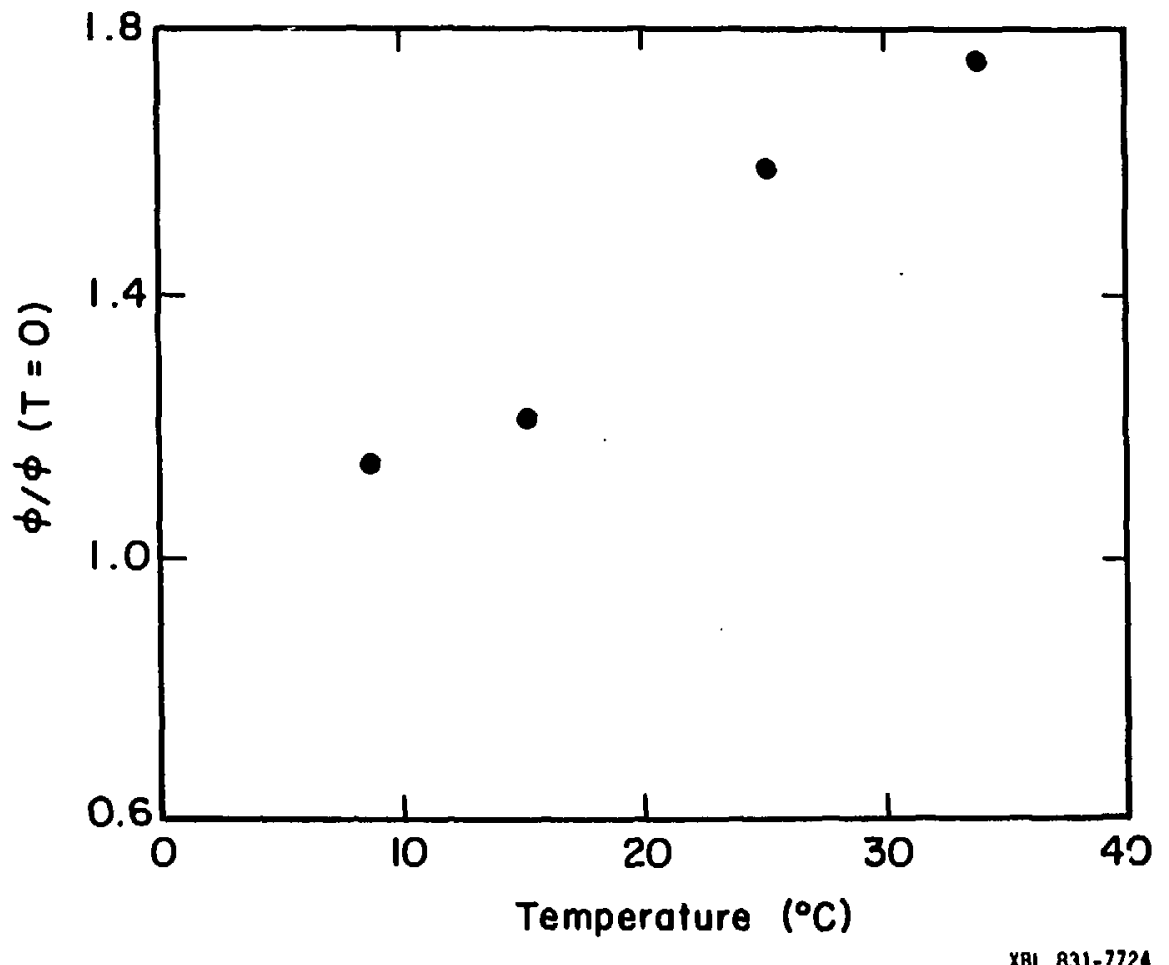

XBL $831-7724$ 
Figure IV-8

The dependence of the quantum yield $(\phi)$ on hydrogen mole fraction for excitation at $9400 \mathrm{~cm}^{-1}$ at $25 \pm 1^{\circ} \mathrm{C}(+)$ and $11800 \mathrm{~cm}^{-1}$ at $24.5 \pm 1^{\circ} \mathrm{C}(0)$. The quantum yield is normalized by the quantum yield in pure $D_{2} \mathrm{O}$ at the excitation wavelength, $\phi\left(D_{2} O\right)$. The values of $\phi\left(D_{2} O\right)$ are $1.93 \times 10^{-7}$ at $9400 \mathrm{~cm}^{-1}$ and $8.12 \times 10^{-7}$ at $11800 \mathrm{~cm}^{-1}$. The solid curve is a fit to the form of

$$
\Phi=\sum_{i} E_{i} c_{i} \& \phi_{i} / \sum_{i}^{E} E_{i} c_{i}{ }^{2}
$$

where $i$ represents $H_{2} O$, HDO, or $D_{2} O$ and $\varepsilon_{i} c_{i}$ is the absorbance of species i. At $9400 \mathrm{~cm}^{-1}$, the best fit is with $\phi_{\mathrm{H}_{2}} \mathrm{O}=3.81 \times 10^{-7}, \phi_{\mathrm{HOD}}=2 \times 10^{-9}$, and $\phi_{\mathrm{D}_{2}} \mathrm{O} 2.54 \mathrm{x}$ $10^{-7}$. At $11800 \mathrm{~cm}^{-1}, \phi_{\mathrm{H}_{2} \mathrm{O}}=1.037 \times 10^{-6}, \phi_{\mathrm{HOD}}=2.62$ $\times 10^{-7}, \phi_{\mathrm{H}_{2} \mathrm{O}}=1.69 \times 10^{-6}$. Estimated uncertainty at $9400 \mathrm{~cm}^{-1}$ is shown in Table III-2. Estimated uncertainty at $11800 \mathrm{~cm}^{-1}$ is approximately three times the values in Table III-2. 


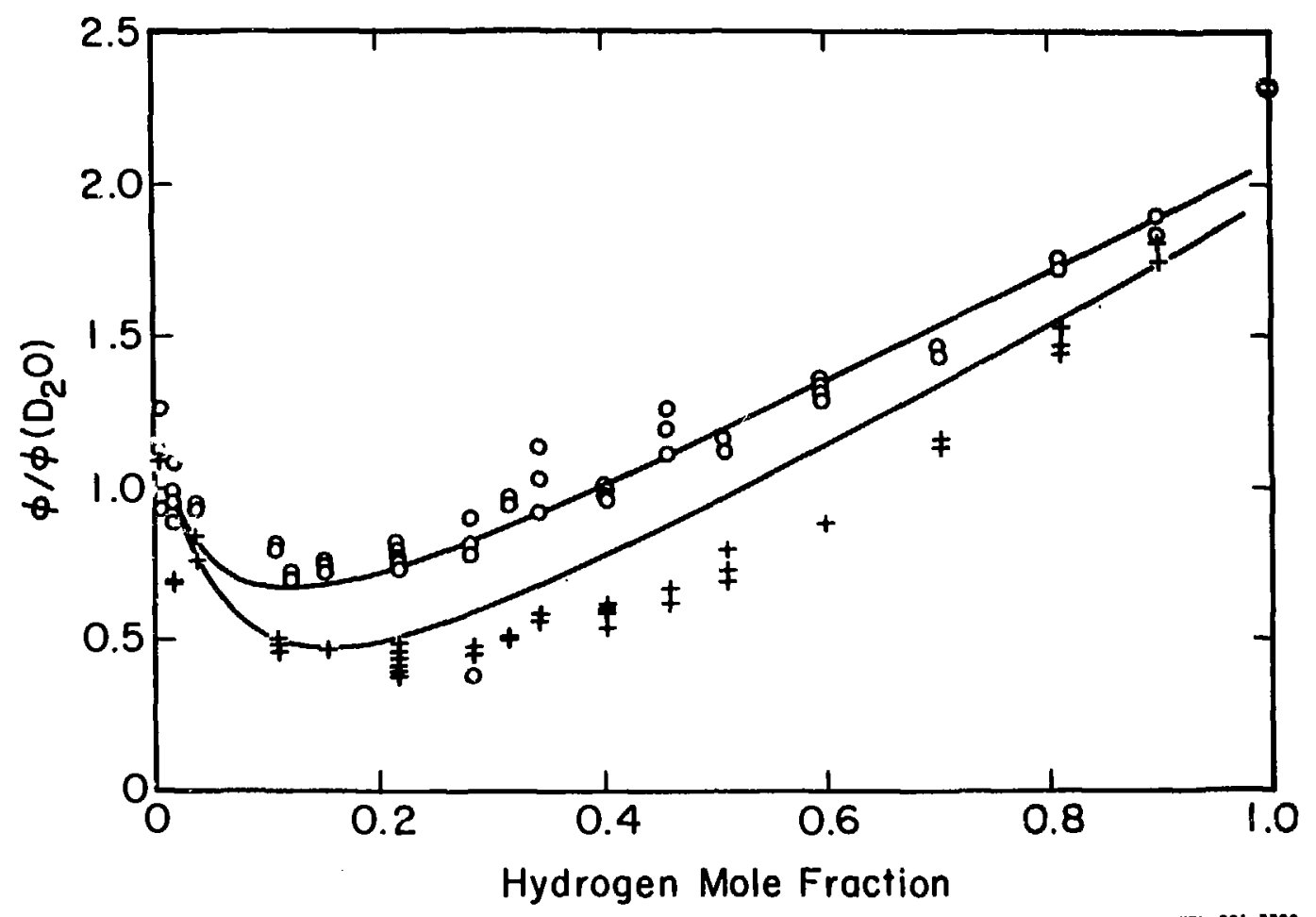

XEL $831-7720$ 
Eigure IV $\rightarrow 9$

The dependence of the quantum yield $(\phi)$ on hydrogen mole fraction for excitation at $15380 \mathrm{~cm}^{-1}$. The quantum yield is normalized by the quantum gield in pure $\mathrm{D}_{2} \mathrm{O},\left(\mathrm{D}_{2} \mathrm{O}\right)$ 


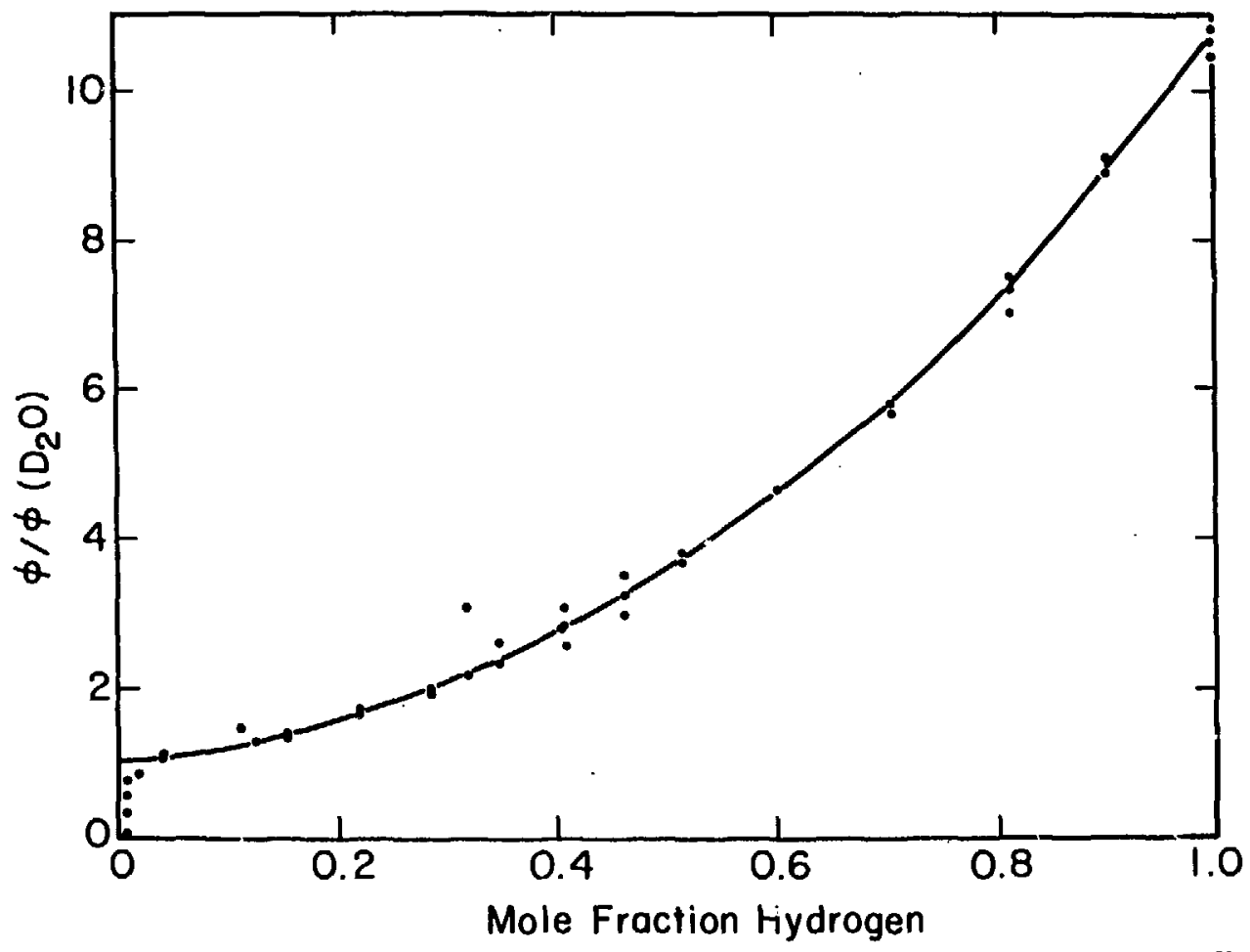

X81 $931-i 721$ 
Figure IV-10

Experimental transient conductivity curves for several hydrogen mole fractions. Excitation is $9400 \mathrm{~cm}^{-1}$ and temperature, $25^{\circ} \mathrm{C}$. The peak amplitude is scaled to equal $I$. A larger baseline step denotes a lower quantum yield, to a first approximation. Curve $A$ has the lowest quantum yield and curve $E$ the greatest quantum yield. Curve $A\left(0.15 x_{H}\right)$, B $\left(0.60 x_{H}\right), C\left(0.81 x_{H}\right), D\left(0.0174 x_{H}\right)$, and $E\left(1.0 x_{H}\right)$. 


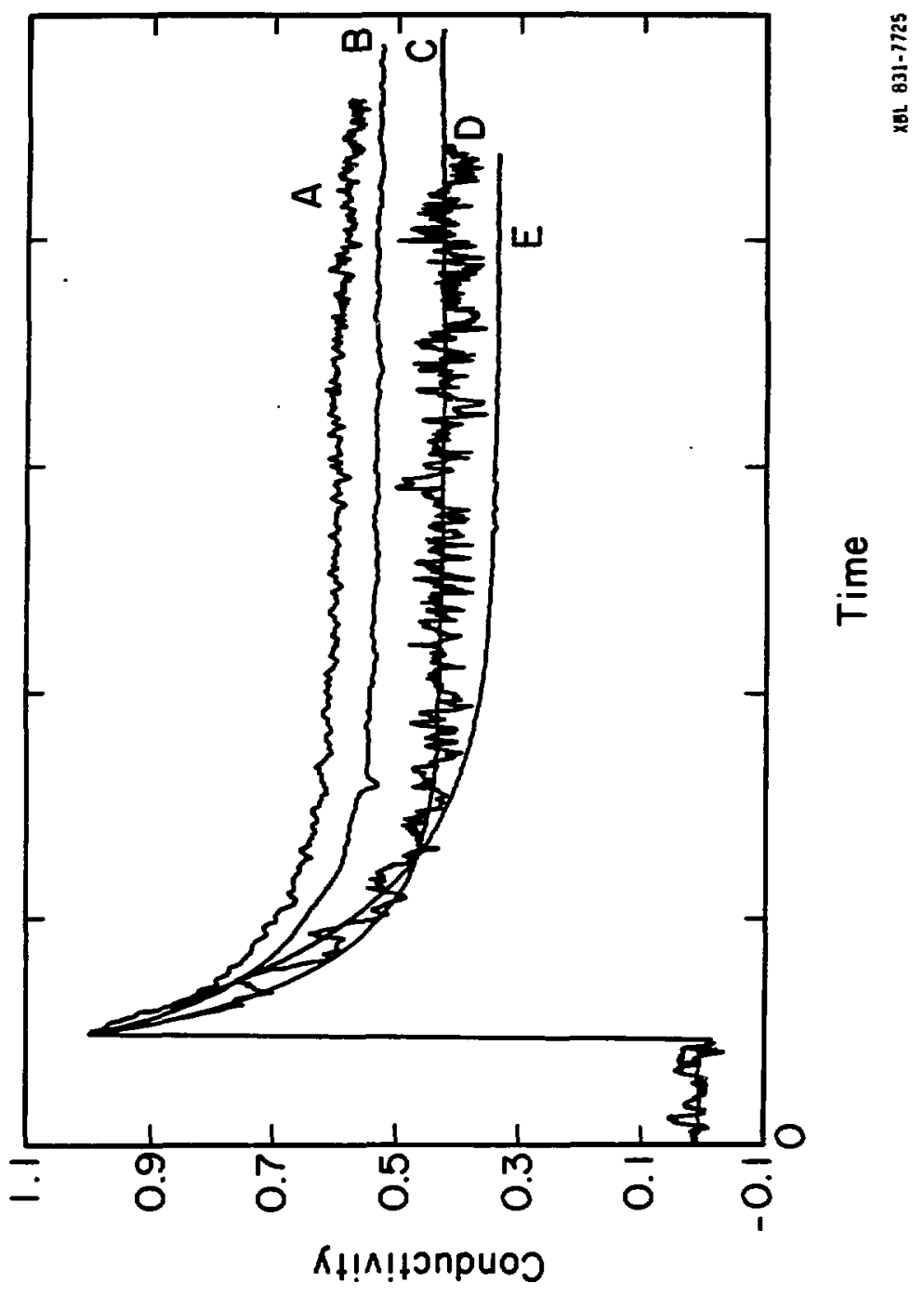


The apparent decline in quantum yield for the point with highest deuterium enrichment is not outside the conbined experimental error for conductivity peak height and spectral absorbance. If the true absorbance of $D_{2} O$ were lower than used in the quantum yield calculation, then the first two or three points in Figure IV-9 would have a higher position. Two factors could produce excess absorbance in the reported $D_{2} O$ absorbances ${ }^{8}$; $\mathrm{H}_{2} \mathrm{O}$ contamination, or organic contaminants in the feedstock $D_{2} O$.

II. Thermal Recombination Reaction

This section presents the thermal recombination rate as a function of temperature and isotopic composition. Figure IV-11 shows the temperature dependence of the rate in $\mathrm{H}_{2} \mathrm{O}$ and in $\mathrm{D}_{2} \mathrm{O}$. An Arrhenius plot, in Figure IV-12 gives $k=3.94 \times 10^{13} \exp (-3.49 / \mathrm{RT})$. In $\mathrm{H}_{2} \mathrm{O}$, the Arrhenius rate increases from $6.37 \times 10^{10} \mathrm{~L} / \mathrm{mol} \mathrm{s}$ at $0^{\circ} \mathrm{C}$ to $1.72 \times 10^{11} \mathrm{~L} / \mathrm{mol} \mathrm{s}$ at $50^{\circ} \mathrm{C}$. The rate at $25^{\circ} \mathrm{C}$ is $1.09 \times 10^{11} \mathrm{~L} / \mathrm{mol} \mathrm{s}$. The Arrhenius line lies slightly below the average of the experimental points at $1.12 \times 1011$ $\mathrm{L} / \mathrm{mol} \mathrm{s}$. $\mathrm{D}_{2} \mathrm{O}$ results are not as accurate due to lower signal amplitudes and fewer measurements. A normal isotope effect is observed with the rate for hydrogen greater than the rate for deuterium. The rate in $\mathrm{x}_{\mathrm{H}}=$ $0.017 \mathrm{D}_{2} \mathrm{O}$ at $25^{\circ} \mathrm{C}$ is $7.07 \times 10^{10} \mathrm{~L} / \mathrm{mol} \mathrm{s}$. Table IV-1 
Eigure IV-11

Thermal ionic recombination rate as a function of temperature for $\mathrm{H}_{2} \mathrm{O}(\bullet)$ and $\mathrm{D}_{2} \mathrm{O}(\mathrm{x})$. 


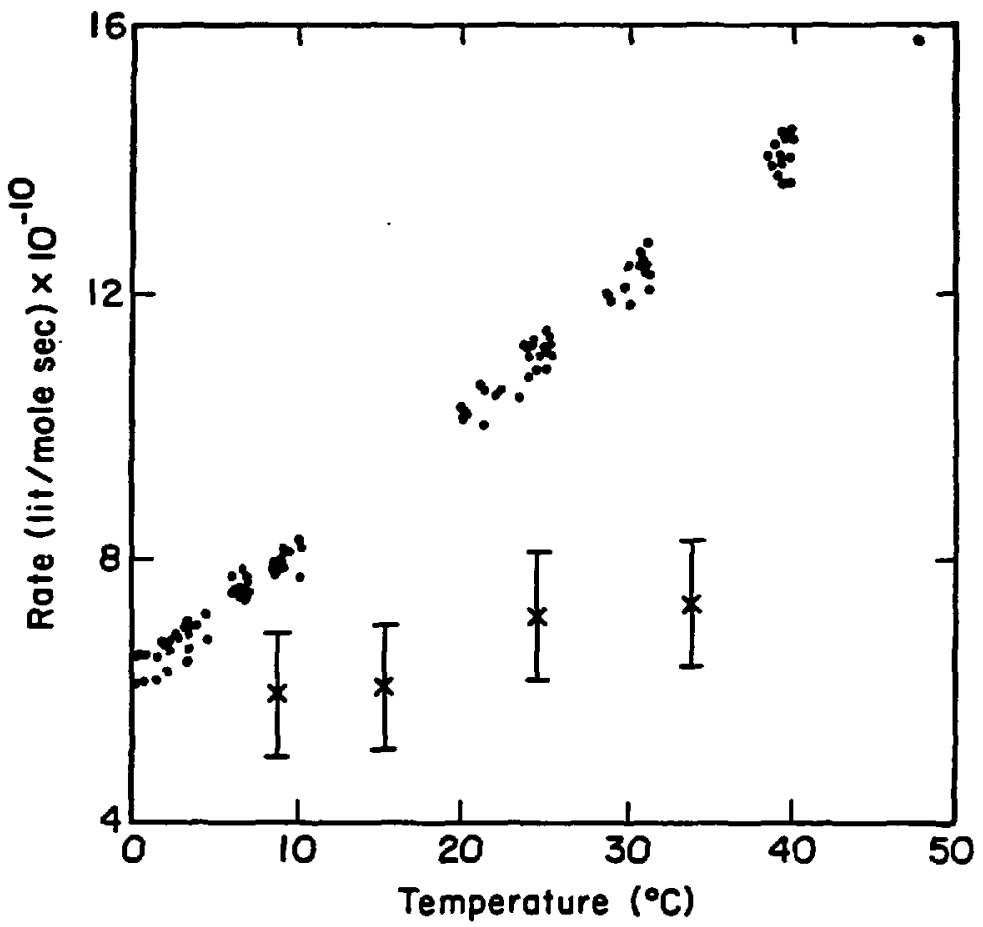

XBL 831-7702 
Figure IV-12

Arrhenius plot of $\ln (k)$ vs $1000 / T$ for the thermal recombination. The solid line $(\rightarrow$ ) is a least squares fit of this data (.). The dashed line (--) is a comparison with reference 9 , the points $(\Delta)$ are from reference 10 . The point $(0)$ is from reference 11 , the point $(x)$ from reference 12, and the point (+) from reference 1 . 


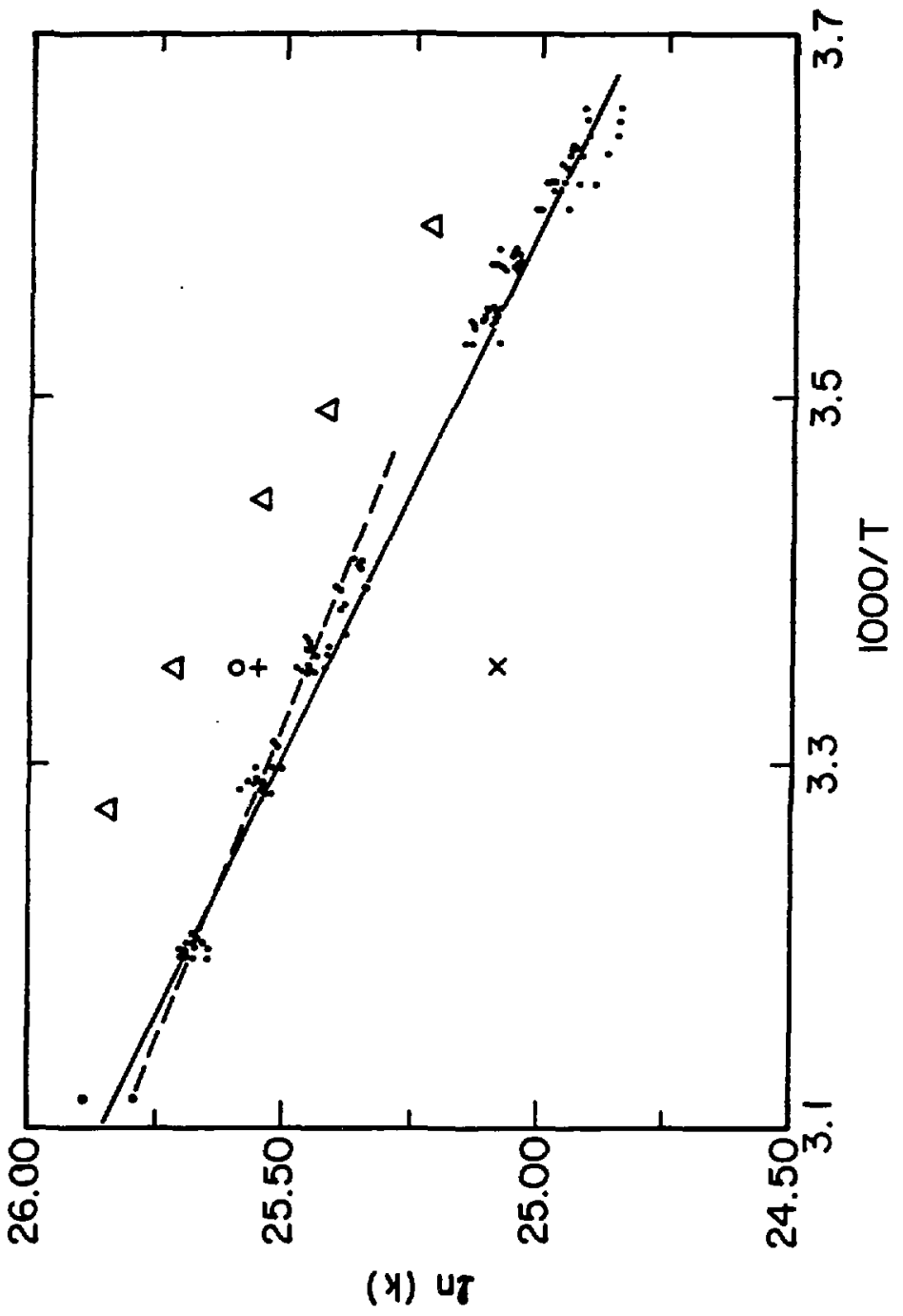




\section{Table IV-1}

Table of recombination rate, relaxation time, and recombination distances in $\mathrm{H}_{2} \mathrm{O}$ and $\mathrm{D}_{2} \mathrm{O}$

recombination

rate

(t/mol s) recombination relaxation distance (A) time (us)

$\mathrm{H}_{2} \mathrm{O}$

$\begin{array}{llcc}\text { Ertl and Gerischer } & 1.46 \times 10^{11} & 34 & 9.2 \\ \text { Eigen and DeMaeyer } & 1.3 \times 10^{11} & 37 & 8.03 \\ \text { Barker et al.c } & 1.12 \times 10^{11} & 44 & 5.85 \\ \text { This work } & 1.12 \times 10^{11} & 44 & 5.85 \\ \text { Knight et al.e } & 1.25 \times 10^{11} & - & - \\ \text { Briere and } & & & \\ \text { Gaspard } & 7.83 \times 10^{10} & 63.5 & \sim 0 \\ D_{2} \mathrm{O} & & & \end{array}$

Ertl and Gerischera $8.9 \times 10^{10}$

150

8.1

This workd

$7.5 \times 10^{10}$

178

6

Recombination distances are recalculated from the relaxation times given by other workers using the ionic conductivity given in the analysis section and the dielectric corstants given in the Discussion.

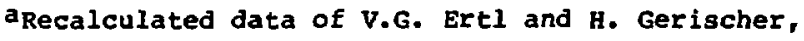

z. Elektrochem. 66, 560 (1962) 


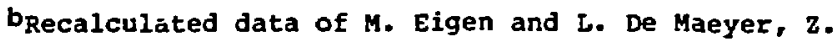
Elektrochem. 59, 986 (1955)

CRecalculated data of G.C. Barker, P. Fowles, D.C. Sammon, and B. Stringer, Trans. Faraday Soc. $\underline{66}, 1498$ (1970)

$d_{D_{2}} \mathrm{O}$ result is estimated from extrapolation of mole fraction dependence to pure $\mathrm{D}_{2} \mathrm{O}$. $\mathrm{H}_{2} \mathrm{O}$ result is not from an Arrhenius fit, it is an average of points near $25^{\circ} \mathrm{C}$.

eB. Knight, D.M. Goodall, and R.C. Greenhow, J. Chem Soc. Faraday 2, 75, 841 (1979)

$f_{\text {Recalculated data of }}$. Briere and E. Gaspard, J. Chim. Phys. 64, 1071 (1967) 
shows a comparison with previous results from both $\mathrm{H}_{2} \mathrm{O}$

and $\mathrm{D}_{2} \mathrm{O}$ at $25^{\circ} \mathrm{C}$. Our results for $\mathrm{H}_{2} \mathrm{O}$ show closer agreement with Barker et al.9 than with the other results10-12.

Figure IV-13 shows the recombination rate as a function of hydrogen mole fraction. There is a monotonic increase from pure $\mathrm{D}_{2} \mathrm{O}$ to $\mathrm{H}_{2} \mathrm{O}$. 
Figure IV-13

Thermal recombination rate as a function of hydrogen mole fraction. Error bars show the range of at least fc values in a weighted least squares average. 


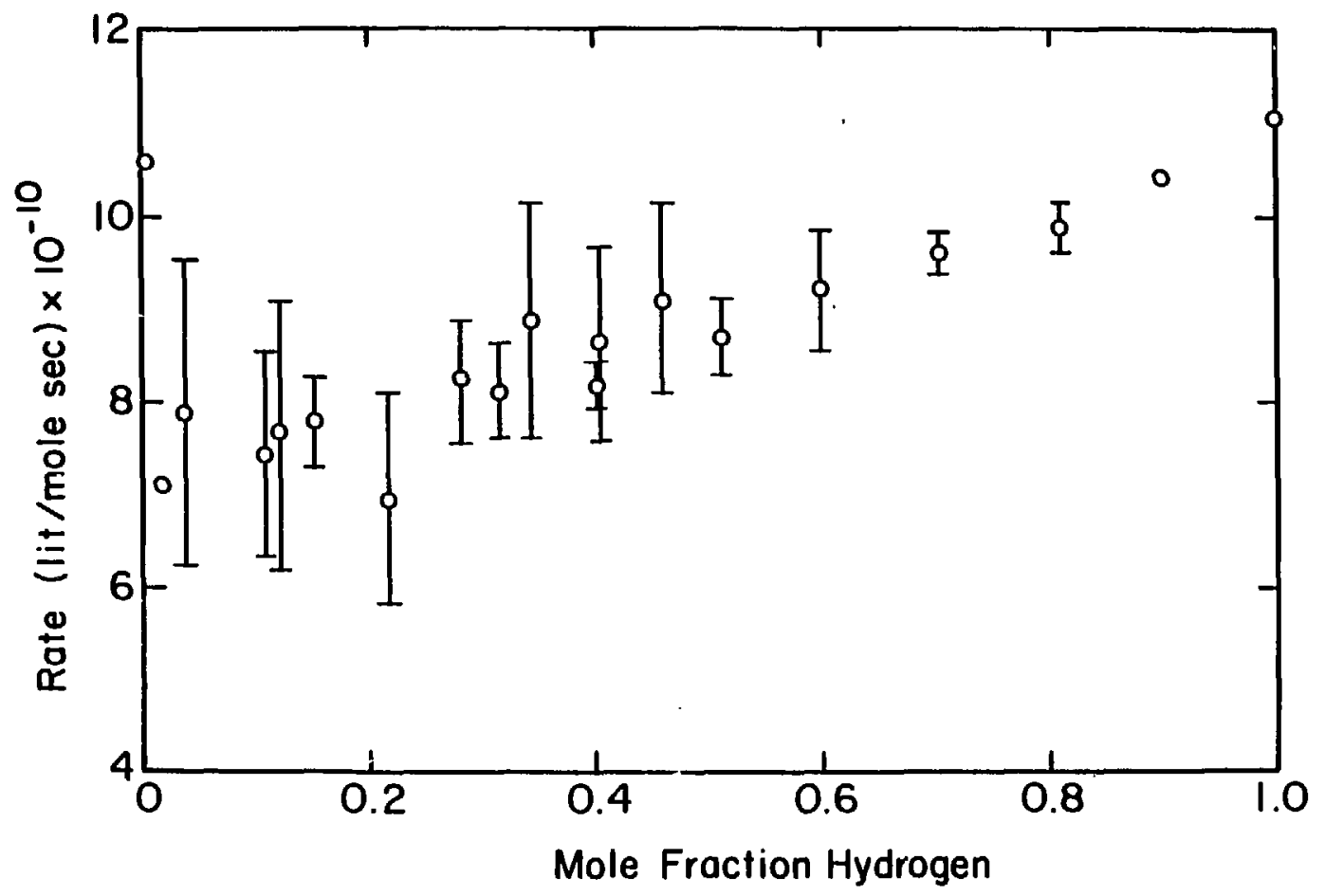

Xㄷ․ $831-7733$ 
I. B. Knight, D.M. Goodall, and R. Greenhow, J. Chem. Soc. Faraday Trans. 2, 75, 841 (1979)

2. C.K.N. Patel and A.C. Tam, Nature (London) 280, 302 (1979)

3. W.h.P. Luck, "Structure of Water and Aqueous Solutions", erlag Chemie (1974) p 251.

4. (.M. Hale and M.R. Querry, Appl. Opt. 12, 3 (1973)

5. M.A.P. Luck and W. Ditter, J. Mol. Struct. 1, 261 $(1967 / 68)$

6. 'A.P. Luck and W. Ditter, Z. Naturforsch. B24, 482 1969)

7. 7. Eisenberg and W. Kauzmann, "The Structure and Properties of Water" (Oxford University Press, New York (1969), p 188

8. t.C. Tam and C.K.N. Patel, Applied Optics 18, 3348 (1979)

9. G.C. Barker, P. Fowles, D.C. Sammon, and B. Stringer, Trans. Faraday Soc. 66, 1498 (197U)

10. G. Ertl and H. Gerischer, Z. Elektrochem. $\underline{66}, 560$ :1962)

11. M. Eigen and L. De Maeyer, Z. Elektrochem. 59, 986 1955)

12. 3. Briere and F. Gaspard, J. Chim. Phys. 64, 1071 (1967) 


\section{CHAPTER V. DISCUSSION}

This section is broken into two parts. First, the thermal recombination rate, then the laser-induced reaction will be examined.

I. Thermal Recombination Reaction

The recombination reaction was first modeled by Eigenl,2 as a diffusion-controlled approach of hydronium and hydroxide ions followed by a rapid neutralization by proton transfer. Ionic diffusion coefficients are combined with recombination rates to yield a recombination distance.

Following Holzwarth et al ${ }^{3}$

$$
k_{-1}=4 \pi N D_{A B} \circ \delta /\left(e^{j}-1\right)
$$

where $k_{-1}$ is the recombination rate, $k$ is Avogadro's number, $D_{A B}$ is the sum of the difiusion coefficients for reacting ions $A$ and $B, \sigma$ is the reaction distance and $\delta$ is given by

$$
\delta=z_{A} z_{B} e^{2 / \sigma \varepsilon k T .}
$$

$\mathrm{z}_{\mathrm{A}} \mathrm{e}$ and $\mathrm{z}_{\mathrm{B}} \mathrm{e}$ are ionic charges, $\varepsilon$ is the $\mathrm{H}_{2} \mathrm{O}$ dielectric constant, $T$ is the temperature and $k$ is Boltzmann's constant. Physically, $\delta$ is the ratio of the coulomb energy at the recombination distance to the thermal energy. Ionic diffusion coefficients are given by the Nernst-Einstein relation

$$
D_{i}=R T z_{i} \lambda_{i} / F^{2}
$$

where $F$ is the Faraday constant and $\lambda_{i}$ is the single ion equivalent conductivity. The dielectric constant for 
$\mathrm{H}_{2} \mathrm{O}$ and $\mathrm{D}_{2} \mathrm{O}$ is given by Vidulich et al. 4 . A linear interpolation was used for intermediate mole fractions. Expressions used for ionic conductivities and recombination rates were given in the analysis section.

The reaction distance calculated from equations (1-3) for $\mathrm{H}_{2} \mathrm{O}$ at $25^{\circ} \mathrm{C}$ is $5.85 \pm 0.5 \mathrm{~A}$. A comparison with previous results is given in Table IV-1. The dependence of reaction distance on terperature and mole fraction of hydrogen is given in Figure V-1. Both Ertl and Gerischer 5 and Barker et al..$^{6}$ report results at temperatures other than $25^{\circ} \mathrm{C}$. The results reported by Ertl and Gerischer show considerable scatter, varying from 5.9 \& to 8.5 \& for five temperatures between $5^{\circ} \mathrm{C}$ ard $32^{\circ} \mathrm{C}$ with perhaps a slight increase in distance with increasing temperature. Barker et al. 6 report a $0.6 \mathrm{~A}$ decrease in distance with a temperature increase from $25^{\circ} \mathrm{C}$ to $50^{\circ} \mathrm{C}$. However, it is not clear what values were used for ionic diffusion coefficients and dielectric constants. The constant reaction distance of $5.8 \pm 0.5 \mathrm{~A}$ with $t$ mperature and isotopic composition observed here is physically more pleasing than the variation suggested pzeviously5,6. The extensive hydrogen bonding in liquid water produces local structure with tetrahedral arrays of $\mathrm{H}_{2} \mathrm{O}$ oxygens having $\mathrm{O} \ldots$... atom distances in neutral water of about $2.8 \mathbf{A}^{7}, 8$. This distance is nearly independent of temperature and isotopic composition of the liquid. Structure need not be broken for transport of hydronium and hydroxide ions through 
the solution. Approxisately 80 of the proton conductivity is caused by an ionic hopping mechaniso where the proton jumps from an $\mathrm{B}_{3} \mathrm{O}^{+}$to the $O$ atom of a neighboring $\mathrm{B}_{2} \mathrm{O}$ molecule ${ }^{9}$. A similar mechanism can account for high hydroxide mobility. The potential energy a proton hopping towards a hydroxide ion feels can be thought of as a superposition of a symmetric barrier midway between equilibrium positions of the oxygen atoms with an attractive coulomb potential. The potential is shown schematically in one dimension in Figure V-2. Thus, the possible ionic recombination distances should be "quantized", with the allowed distances determined by the location of successive 0 atom wells. Neutron diffraction studies show that the distance is considerably compressed in the hydronium and hydroxide ion relative to neutral water 10 with an o -.. o distance of about $2.52 \mathrm{~A}^{11}$ for hydronium in either $\mathrm{H}_{2} \mathrm{O}$ or $\mathrm{D}_{2} \mathrm{O}$. Bond distances in crystals containing hydronium ion hydrates $12-15$ and theoretical studies of hydrated hydronium ions 16 also show a similar $0 . .0$ atom distance. Theoretical studies of hydroxide ions 16 wi th a successively increasing number of water molecules of hydration show an $0 \cdots 0$ distance between $2.45 \mathrm{~A}$ and 2.61 A. This distance is somewhat larger than for the corresponding hydronium ion but still greatly compresssed relative to the neutral water distance. Crystal structures containing hydroxide ion hydrates 17,18 also 
Figure $\mathrm{v}-1$

Recombination distance as a function of temperatue $(X)$ in $\mathrm{H}_{2} \mathrm{O}$ and mole fraction hydrogen $(\mathrm{O})$ at $25^{\circ} \mathrm{C}$. The recombination distance is constant within experimental error with temperature and mole fraction hydrogen. The solid line is a recombination distance of $5.8 \mathrm{~A}$. 


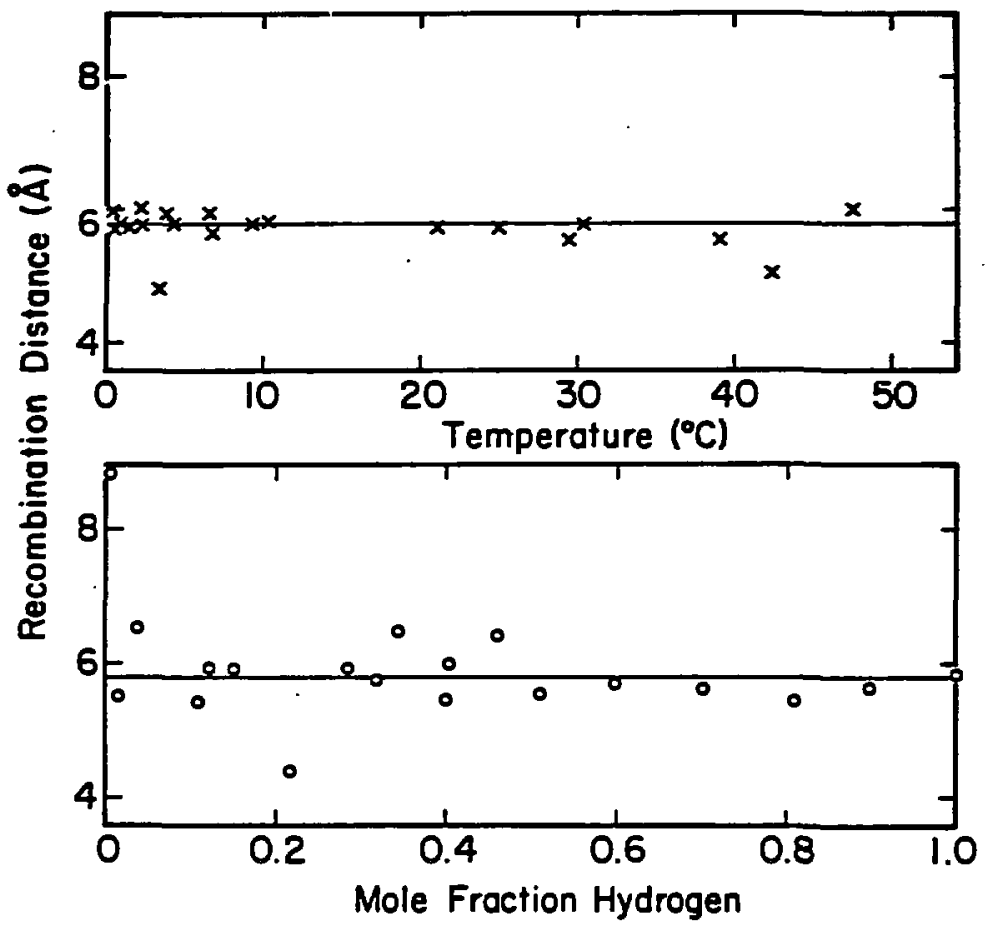

XeL 631-P7os 
Figure v-2.

one-dimensional schematic diagram of the potential of a proton in a linear chain of hydrogen bonded oxygen atoms (A) : the Coulomb attraction of a proton to a hydroxide ion (B); and (C), the superposition of potentials (A) and (B). 


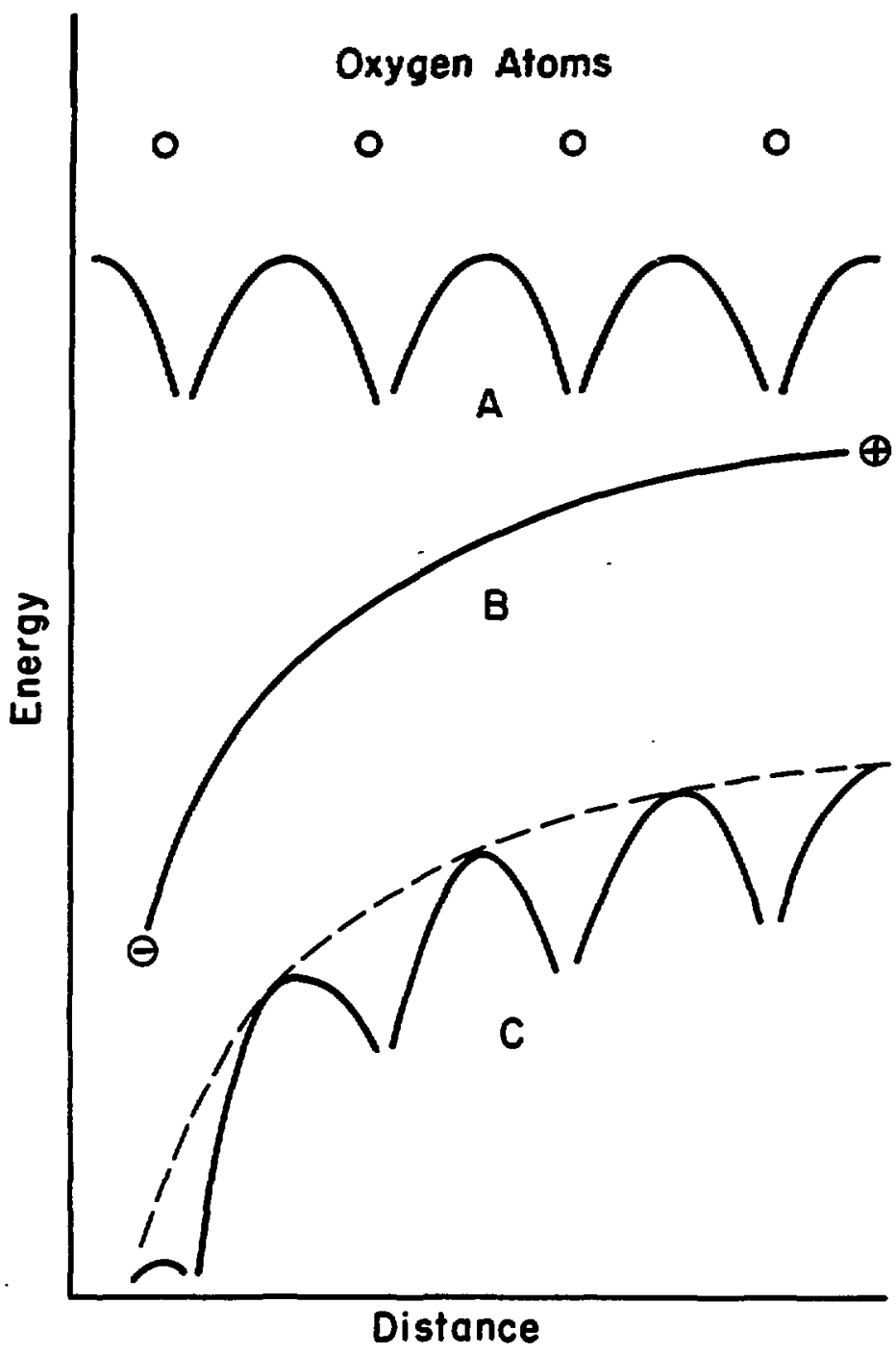

XBL 831-7705 
show a slightly longer $0 . . .0$ distance than for the corresponding hydronium ions.

Eigenl,2 first proposed a linearly bound, four oxygen, ion pair chain intermediate for the ionic recombination. This intermediate will have a configuration-dependent "allowed" distance for the ionic ends of berween $6.3 \AA$ and $4.2 \AA$. This assumes a 2.52 nearest neighbor oxygen atom separation similar to ions rather than the 2.8 A for neutral water. The ion pair H-O ... E bond angle is $109^{\circ}$. The measured recombination distance is between these two extremes. The distance is not expected to vary strongly with temperature or isotopic composition because the 0-0 atom separation does not change markedly. Figure $\mathrm{V}-3$ shows the geometry for the two extreme configurations. In conclusion, the observed recombination distance and lack of temperature and isotopic dependence is consistent with the linear four-molecule chain intermediate.

\section{Laser Reaction}

One goal of the Discussion is to give a physically meaningful interpretation of the results. The discussion of the laser-induced reaction consists of two parts. The first part is a physical interpretation that is independent of the model used to describe the quantum yield kinetics. The second part is a formulation and application of a guantum yield model. Many of the quaistum yield results cannot be given a physically meaningful interpretation without development of a model. 
Figure $v-3$

The minimum and maximum distance geometry for a linear chain of four hydrogen-bonded water molecules. 

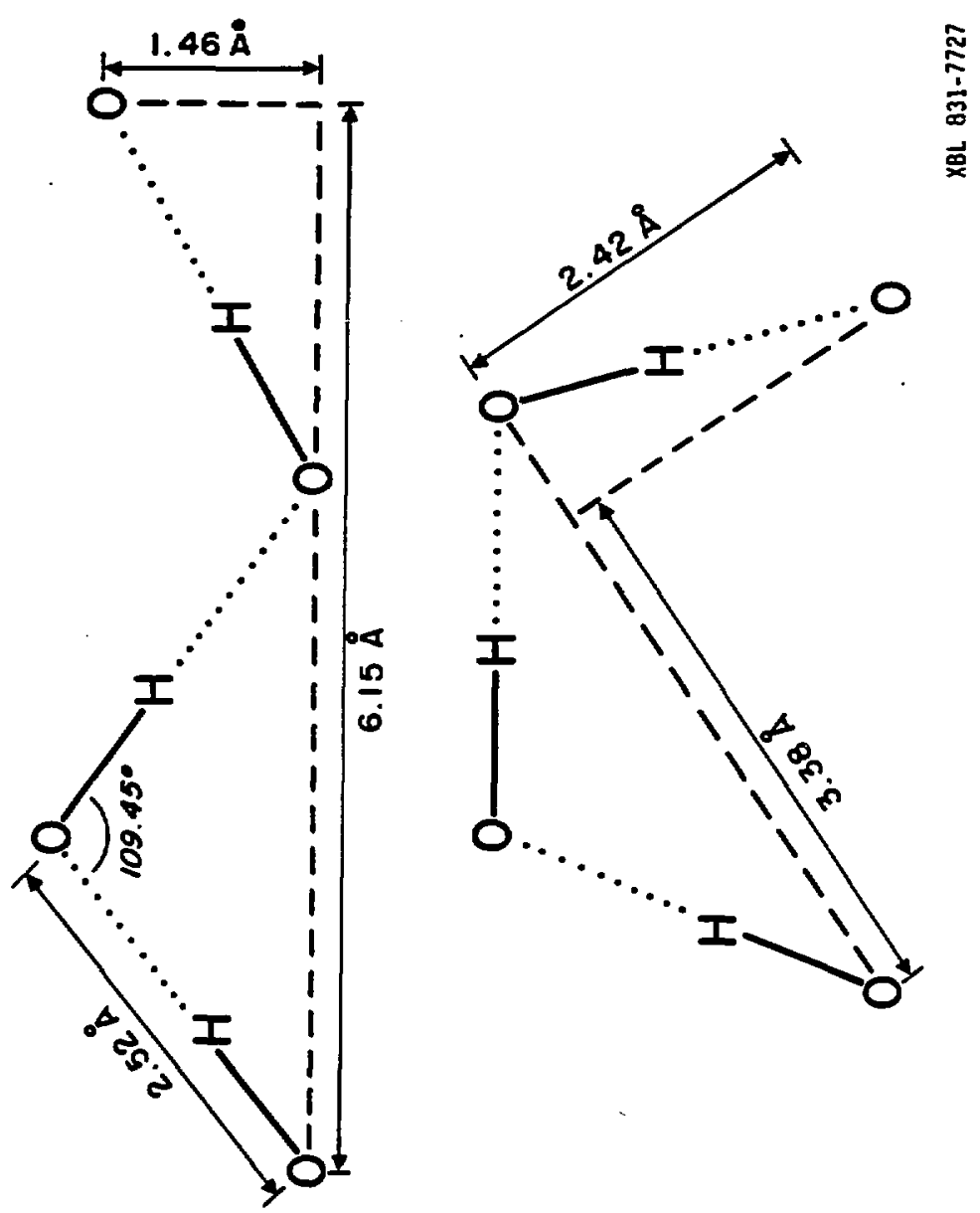
A. Model Independent Interpretation

1. Nature of the laser-induced reaction.

The experimental results demonstrate that the laser reaction is induced by vibrational overtone absorption of a single photon in liquid water to produce $\mathrm{H}_{3} \mathrm{O}^{+}$and $\mathrm{OH}^{-}$ions. The evidence is as follows: The linearity and unit slope of the conductivity peak vs. pulse energy plots of Figures IV-1 and IV-2 denote a single photon process. Agreement of the measured relaxation rate with previous measurements of the $\mathrm{H}_{3} \mathrm{O}^{+}$ and $\mathrm{OH}^{-}$equilibrium relaxation rate (Table $\mathrm{IV}-1$ ) is consistent with $\mathrm{H}_{3} \mathrm{O}^{+}$and $\mathrm{OH}^{-}$final products. Signal from an impurity absorption would not correlate with the $\mathrm{H}_{2} \mathrm{O}$ overtone absorption spectrum, contrary to data shown in Figures IV-3, IV-4, and IV-5. Additional evidence that an impurity does not produce the conductivity signal is provided by the absolute magnitude and the relative magnitude of the conductivity peak in $\mathrm{H}_{2} \mathrm{O}$ and $\mathrm{D}_{2} \mathrm{O}$. An impurity would absorb photons by making either an overtone transition or an electronic transition. Estimates of the magnitude of the peak conductivity in each case follow.

The first possibility is overtone absorption into an A-H stretch of an impurity molecule, A-H. If the absorption strength of the $\mathrm{A}-\mathrm{H}$ bond were the same as the $\mathrm{O}-\mathrm{H}$ stretch of an $\mathrm{H}_{2} \mathrm{O}$ and the reaction quantum yield were 1 , then the 
impurity level would have to be about $40 \mathrm{ppm}$ to produce the observed quantum yield of $4 \times 10^{-5}$ at $18,140 \mathrm{~cm}^{-1}$. A more. -stic, lower estimate of the quantum yield would require a $>40 \mathrm{ppm}$ impurity concentration. No impurities were observed in a GCMS at a sensitivity of $5 \mathrm{ppm}$. Therefore, a vibrational transition by an impurity could not produce the conductivity signal.

The second possibility is electronic absorption of an impurity to produce an excited electronic state with lower pK than the ground state. The absorption strength would be considerably larger for an electronic absorption than for an overtone absorption. An impurity could be present at a level < 5 ppm and still produce the observed signal. However, the relative conductivity peak magnitude observed upon changing the solvent from $\mathrm{H}_{2} \mathrm{O}$ to $\mathrm{D}_{2} \mathrm{O}$ eliminates the possibility that electronic absorption by an impurity caused the transient conductivity. Solvent change does not affect the electronic absorption strength of the impurity, but could cause a signal decrease due to both ionic mobility and reaction rate or equilibrium isotope effects. The maximum peak conductivity can be estimated. Kinetic and equilibrium isotope effects lie in a range from 1.5-1119. An isotope effect of 11 coupled with the mobility decrease upon a solvent change from $\mathrm{D}_{2} \mathrm{O}$ to $\mathrm{H}_{2} \mathrm{O}$ produces at most a factor of 16.5 reduction in the conductivity peak height. The measured peak height reduction is by a factor of 60-70. 
2. Wavelength dependence of the quantum yield in $\mathrm{H}_{2} \mathrm{O}$

Interpretation of the constancy of quantum yield with wavelength within an absorption. band requires knowledge of the main source of band broadening. Two broadening mechanisms for $0-\mathrm{H}$ or $\mathrm{O}-\mathrm{D}$ stretch fundamentals and first overtones in the solid or liquid water will be discussed. The mechanisms are (1) inhomogeneous broadening caused by a random, slowly-changing distribution of $0 . . .0$ distances of neighboring H-bonded molecules; and (2) a combination overtone series caused by strong coupling of $\mathrm{O}-\mathrm{H}$ stretch motions with $0 \cdots 0$ stretch vibrations.

Rice and Sceats 20 have developed a model for liquid water based mainly on spectroscopic measurements of tie liquid and of the crystalline, or amorphous solid. The model was used by Sceats and Belsley2l to interpret the overtone OH stretching spectrum. First, the fundamental $\mathrm{O}-\mathrm{H}$ stretch was examined. A total width caused by a temperature-dependent homogeneous and a temperatureindependent inhomogeneous portion is calculated for the ice I $\mathrm{O}-\mathrm{H}$ stretch spectrum of $\mathrm{HOD}$ in $\mathrm{D}_{2} \mathrm{O}$. The local structure and spectral features caused by librational and translational motions in the liquid are similar to ice. Since these motions are responsible for the homogeneous spectral width in ice, the same 
temperature-dependent width was assumed for the liquid. A root mean square homogeneous width increasing from $28 \mathrm{~cm}^{-1}$ to $42 \mathrm{~cm}^{-1}$ between $10^{\circ} \mathrm{C}$ and $90^{\circ} \mathrm{C}$ was found for the liquid. The calculated homogeneous width is much smaller than the measured spectral kidths; consistent with their hypothesis that the dominant contribution to the $0-H$ stretch lineshape arises from inhomogeneous broadening. Sceats and Belsley conclude that the homogeneous component of the overtone $\mathrm{O}-\mathrm{H}$ stretch should be only slightly larger than for the fundamental. The Sceats and Belsley model assumes the $\mathrm{O}-\mathrm{H}$ stretch absorption band is a "pure" band, not in combination with other liquid motions.

The second broadening mechanism involving a combination of $0-H$ stretch with the $0-\mathrm{H} \cdots 0$ stretch of hydrogen-bonded oxygens has recent experimental support22-27. This mechanism has been used successfully for interpretation of spectra of other hydrogen-bonded systems. References 24 through 27 describe experimental and theoretical work on the gas phase $\mathrm{Me}_{2} \mathrm{O} \cdots \mathrm{HCl}$ and $\mathrm{Me}_{2} \mathrm{O}$-.. HF systems. The spectra are adequately described in a model using a Born-oppenheimer type separation of the rapid $\mathrm{X}-\mathrm{H}$ stretch motion from the slower $\mathrm{X}-\mathrm{H} \ldots \mathrm{y}$ hydrogen bond motion. The $\mathrm{XH} \cdots$... are determined by an effective potential, that is dependent upon the quantum state of the $x-H$ oscillator. When a spectroscopic transition is induced in the $\mathrm{x}-\mathrm{H}$ oscillator, the quaneum state of the XH ... Y oscillator 
will change simultaneously if a skift in the effective potential minimum causes a favorable "Franck-Condon" Eactor.

A similar model can be invoked to explain the infrared spectrum of hydrogen-bonded clusters of gas phase water molecules 22 . There are several peaks in the fundamental 0-4 stretching region separated by $150-200 \mathrm{~cm}^{-1}$, the approximate $\mathrm{OH}$... O stretching frequency. The same pattern is observed in the depolarization CARS spectrum of liquid water ${ }^{23}$. This technique enables resolution of the underlying spectral features which compose the fundamental $\mathrm{O}-\mathrm{H}$ stretch absorption, in contrast to normal infrared or Raman spectroscopy where the fundamental has a single broad unresolved peak.

There is a generally accepted precedent in a different condensed phase system for using combination bands of an intramolecular vibration with lattice motions to explain infrared spectral lineshapes 28 . The infrared lineshapes of impurity molecules embedded in rare gas matrices typically show a sharp "fundamental" zero phonon line accompanied by a broad, blue shifted, phonon sideband. The sideband is composed of a combination of the "fundamental" motion with latice phonons. If the lattice phonons are strongly coupled to the fundamental, then the zero phonon line is weak and the phonon sideband dominates the spectrum. The broad absorption lines in water could 
be analogous to the phonon sideband of a matrix isolated molecule strongly-coupled to lattice motions.

Armed with two possible spectral broadening mechanisms, we can use each model to interpret the constant quantum yield within water absorption bands. If the bulk of the broadening is inhomogeneous, as suggested by the Sceats-Rice-Belsley20,21 model, then the quantum yield, $\phi$, is independent of the differences in regional structure which cause the spectral broadening. Since $\phi$ - $\mathrm{k}_{\text {react }} / \mathrm{k}_{\text {relax, }}$ either the ratio $\mathrm{k}_{\text {react }} / \mathrm{k}_{\text {relax }}$ is independent of regional stiucture, or the system remains activated long enough to thermally sample the average regional structure. Rice and Sceats 20 mention a $10^{-11}$ second order of magnitude time for the thermal diffusion processes that alter regional structures.

If brc-dening comes from combination of $\mathrm{OH} \cdots$... lattice motion with the $\mathrm{O}-\mathrm{H}$ stretch, then excitation of the low frequency lattice mode does not produce reaction products as efficiently as an equivalent energy of $\mathrm{OH}$ stretch. Either, the low frequency $\mathrm{OH}$... O stretch is not strongly coupled to the reaction coordinate ( $k_{\text {react }}$ is nearly constant with increasing excitation of the OH ... O) stretch; or energy in the OH ... O stretch is rapidly delocalized and thermalized by strong coupling to surrounding $\mathrm{H}_{2} \mathrm{O}$ molecules ( $\mathrm{k}_{\mathrm{relax}}$ is greater for excitation of the OH $\cdots$ O stretch). 
It is reasonable to expect $O H$... O stretch lattice vibrations to be delocalized rapidly relative to $0-H$ stretch vibrations. The lattice vibrations come from an oxygen atom moving with respect to its neighbors, whereas the OH stretch is predominantly an intramolecular motion.

3. Quantum yield temperature dependence.

A temperature increase raises the quantum yield by increasing the rate of ion pair formation and/or the rate of ionic separation from the ion pair.

Results at several photon energies show that a temperature increase is less effective in promoting the laser reaction as the photon energy goes up. The thermal fraction of the total energy is smaller as the photon energy increases.

4. Hydrogen mole fraction dependence of the quantum: yield and the wavelength dependence in nearly pure $\mathrm{D}_{2} \mathrm{O}$.

A hypothesis that the quantum yield for HOD is small compared to the quantum yield for $\mathrm{D}_{2} \mathrm{O}$ in a predominantly $\mathrm{D}_{2} \mathrm{O}$ solution is consistent with the experimental results shown in Figures IV-8 and IV-5. First, the quantum yield dependence with mole fraction of hydrogen of Figure IV-8 is considered. 
As the mole fraction of added hydrogen increases, the compcsition and the concentration of each excited species changes. The absorption strength of an $\mathrm{O}-\mathrm{H}$ stretch is more than an order of magnitude greater than an $O D$ stretch for wavelengths between $9400 \mathrm{~cm}^{-1}$ and $15,380 \mathrm{~cm}^{-1}$. Upon addition of $\mathrm{H}_{2} \mathrm{O}$ to $\mathrm{D}_{2} \mathrm{O}$, the major excited species in the solution rapidly switches from $\mathrm{D}_{2} \mathrm{O}$ to HOD, the predominant hydrogenated species present at small hydrogen mole fraction. There is an equal absorption into $\mathrm{OH}$ and $\mathrm{OD}$ stretches at 0.06 , at 0.04 , and between 0.01 and 0.06 mole fraction hydrogen for excitation at $9400 \mathrm{~cm}^{-1}, 11800 \mathrm{~cm}^{-1}$, and $15380 \mathrm{~cm}^{-1}$, respectively. Figure $\mathrm{V}-4$ shows the relative absorbance of $\mathrm{H}_{2} \mathrm{O}, \mathrm{D}_{2} \mathrm{O}$, and HOD as a function of hydrogen mole fraction for excitation at $9400 \mathrm{~cm}^{-1}$. The dips in the quantum yield vs. mole fraction hydrogen $C_{L}$.ves of Figure IV-8 are in regions where the absorption is sh1L:ing from $D_{2} O$ to HOD. Figure IV-8 includes a fit of the quantum yield vs. mole fraction hydrogen to the form

$$
\phi=\frac{\sum_{i} \varepsilon_{i} \phi_{i}}{\sum_{i} \varepsilon_{i}} .
$$

Species " $i$ " represents $\mathrm{H}_{2} \mathrm{O}, \mathrm{D}_{2} \mathrm{O}$, or HOD. The base 10 absorbance of species $i$ in the isotopic mixture is $E_{i}$. For excitation at $9400 \mathrm{~cm}^{-1}$, the fit corresponds to a linear combination of the curves in Figure $\mathrm{V}-4$. The best fit at both $11800 \mathrm{~cm}^{-1}$ and $9400 \mathrm{~cm}^{-1}$ is with an 
HOD quantum yield less than either $\mathrm{H}_{2} \mathrm{O}$ or $\mathrm{D}_{2} \mathrm{O}$. The curve from equation (4) has the correct gualitative shape, with a better fit at $11,800 \mathrm{~cm}^{-1}$ than at $9400 \mathrm{~cm}^{-1}$. However, the curve for greater than 0.5 mole fraction hydrogen does not have as much upward curvature as the experimental points.

Assumption of a smaller quantum yield for HOD than for $D_{2} O$ can also reproduce the guantum yield dip on the blue side of the absorption band shown in Figure IV-5. The absorption maximum for HOD peaks on the blue side of the $\mathrm{D}_{2} \mathrm{O}$ band. A 0.017 mole fraction hydrogen mixture will have 508 OD stretch absorption and 508 OH absorption at the $\mathrm{OH}$ stretch maximum. On the red side of the band, the OD stretch is dominant with 758 absorption and the OH stretch contributes $25 \%$. Thus, as the excitation sour ee is tuned from the red to the blue side of the band, increasing amounts of HOD relative to $D_{2} \mathrm{O}$ are excited. Equation (4) can predict the quantum yield behavior. If a relatively small HOD quantum yield of $1.8 \times 10^{-8}$ is assumed, then a $D_{2} \mathrm{O}$ quantum yield of $1.8 \times 10^{-7}$ is required to match the observed $1.38 \times 10^{-7}$ quantum yield for excitation on the red side of the band at $9450 \mathrm{~cm}^{-1}$. The predicted quantum yield on the blue side where the OH stretch has maximum absorbance is $9 \times 10^{-8}$. This agrees quite well with the measured $8.4 \times 10^{-8}$. 
Figure $\mathrm{V}-4$

The relative absorbance of $\mathrm{H}_{2} \mathrm{O}(----), \mathrm{D}_{2} \mathrm{O}(-)$, and HOD (-•-.--.) as a function of mole fraction hydrogen for excitation at $9400 \mathrm{~cm}^{-1}$. 


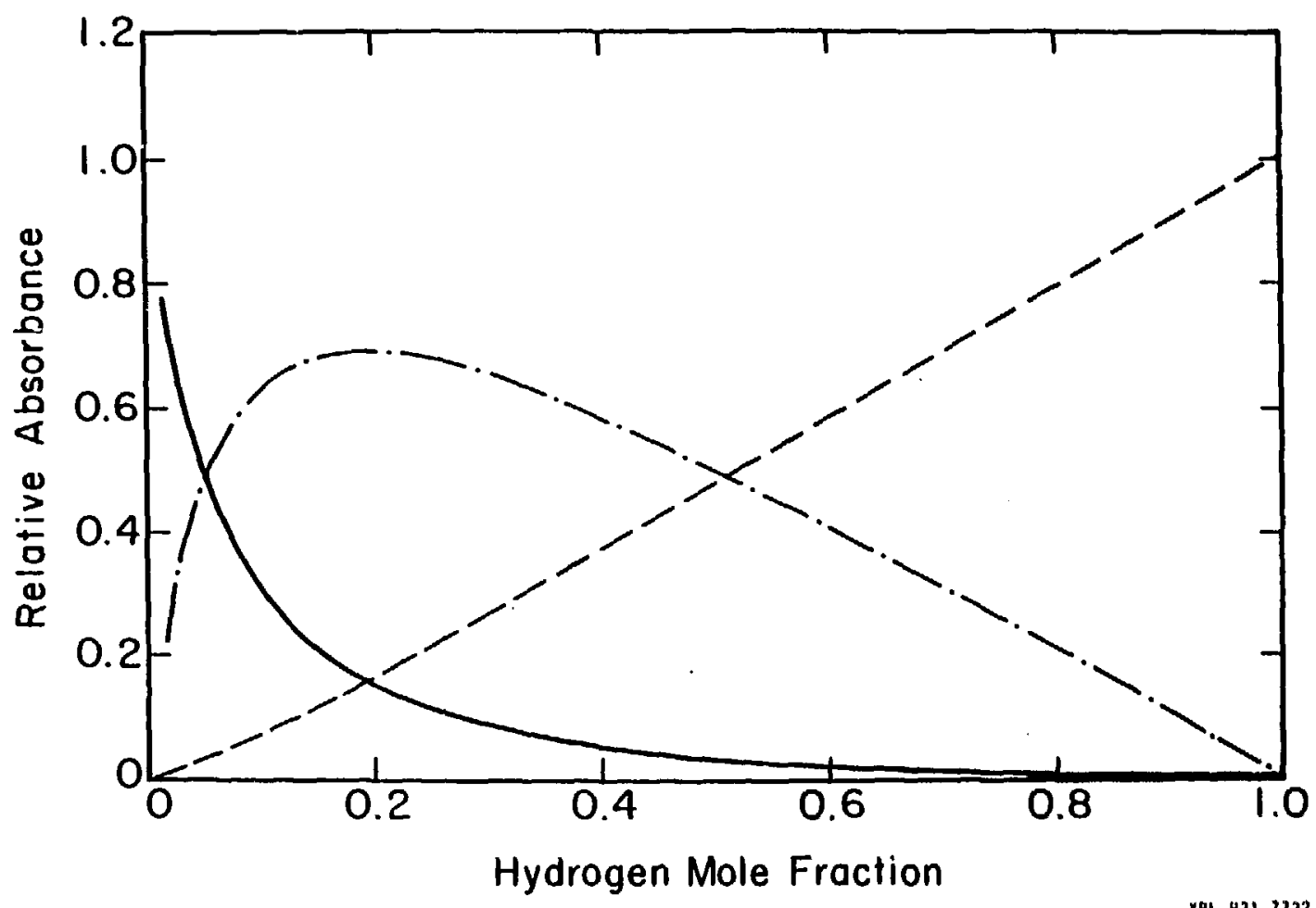

X8L 831.7132 
The question of why the quantum yield for HOD is less than for $\mathrm{H}_{2} \mathrm{O}$ arises. Isotope effects in chemical reactions of isolated gas phase systems are well characterized 29 . Sutstitution of $D$ for $B$ atoms in a reacting system lowers the zero point energy of both the reactant and the transition state. The usual result is an increase in activation energy and a decrease in reaction rate. The experimental measurement of isotope effects in thermal proton transfer reaction rates and equilibria in condensed phase have gained increasing interest ${ }^{30}$.

Typically, rates are not linear, but are monotonically increasing with hydrogen mole fraction, in contrast to the quantum yield results. There are two qualitative differences between the isotopic dependence of a quantum $y$ ield and of a reaction rate. The non-monotonic behavior of the water ionization dissociative quantum yield must have its origin in these differences. First, the overall quantum yield isotope effect depends on the individual isotope effects for several elementary processes including (a) the diffusive separation of ions from the ion pair, (b) the inn pair formation, and (c) the pathway and rate of energy relaxation. Secondly, the activated system is not in equilibrium. Its energy is much greater than the typical thermal energy and is not statistically distributed immediately following 
photon absorption. The O-I stretch motion of bonds containing hydrogen are preferentially excited in isotopic mixtures. Finding the source of the quantur yield mole fraction dependence requires development of a model for the individual elementary processes.

B. Quantum yield model

This section develops a model for the ion formation mechanism. The goal is to provide a qualitative interpretation of the experimental results. So far, the discussion is independent of the model chosen for the reaction and relaxation processes taking place following overtone excitation. The quantum yield described in the analysis section will be discussed here as arising from two consecutive processes. The first process is (a) formation of an ion pair in competition with deactivation of the excited molecule or group of molecules involved in the transition state. This is followed by the second process, (b) diffusive separation of the inn pair to form free ions in competition with recombination. The modeling approach is to divide the ionization processes into two parts with one part dependent on only bulk properties of the solvent and another part dependent on only the local properties of an activated reactant. The function of the division is to remove the contribution from bulk diffusion which has been established and well characterized by thermal recombination work from 
the local rate process that could not be observed in the previous work. Thus, new information about the local rate processes will be obtained. Thermal recombination studies provide no kinetic information for the local ion pair recombination because diffusion to form the ion pair is rate determining. Large changes in ion pair recombination rates will not affect the observed thermal relaxation rate. In contrast, the quantum yield of the laser-induced reaction is dependent on both the efficiency of ion pair separation and the local relaxation, ion pair formation and recombination rates.

Thus, the quantum yield is described by

$$
\phi=P_{I P} \text { PIS. }
$$

The probability of ion pair formation, $P_{I P}$, is given by the competition of ion pair formation with relaxation, $P_{\text {IP }}=k_{1 a} /\left(k_{1 a}+k_{2}\right)$. (See the analysis section for a definition of the rates.) $P_{I P}$ is dependent only upon local reaction and relaxation rates of the activated reactant. The probability of ion pair separation, PIS, is composed of a bulk and a local contribution.

1. Probability of ion pair separation.

The overall energetics for ion pair formation and separation is shown in Figure $\mathrm{V}-5$. In order to contribute to the conductivity signal, an ion pair resulting from the laser-induced reaction must diffuse apart; out of the attractive Coulomb well. 
The standard treatrent for diffusive encounters in the recombination of reactive species was first described by Smoluchowski31,32 and solved for a coulomb potential by Onsager33. The solution, assuming an infinite recombination velocity at the recombination distance was applied to the thermal recombination as described in the first discussion section. The assumption is a reasonable approximation for the diffusion controlled thermal recombination, but not for the ionic separation following the laser-induced reaction. An infinite recombination velocity gives a quantum yield of zero. More recently, a solution with finite recombination velocity was provided by flong and Noolandi34. They give an expression for the ionic survival probability of an isolated ion pair as a function of $(i)^{\prime}$ time following the initial ion formation, (ii) recombination distance, and (iii) formation distance. The conductivity a microsecond or two following excitation is related to the survival probability at long time for an ion pair formed at the recombination distance. The geminate recombination, or recombination of the ion pair, is fast on the timescale of the Hong and Noolandi survival probability and only the ions that permanently escape geminate recombination contribute to the conductivity peak. Then, the quantity of interest for modeling the quantum yield is the survival probability at infinite time given initial formation at a radius equal to the recombination distance. 
Figure V-5

Energetics of ion pair formation and ion pair separation to form free ions. The photon energy is greater than the net endothermicity. The species present in the solution at each energy is shown at the right. A linear four-molecule hydrogen-bonded chain, the ion pair, and free ions are shown. Approximate energies are for $\mathrm{H}_{2} \mathrm{O}$ near $5^{\circ} \mathrm{C}$. The $5.8 \mathrm{~A}$ distance denotes the distance between oxygen centers in the ion pair. 


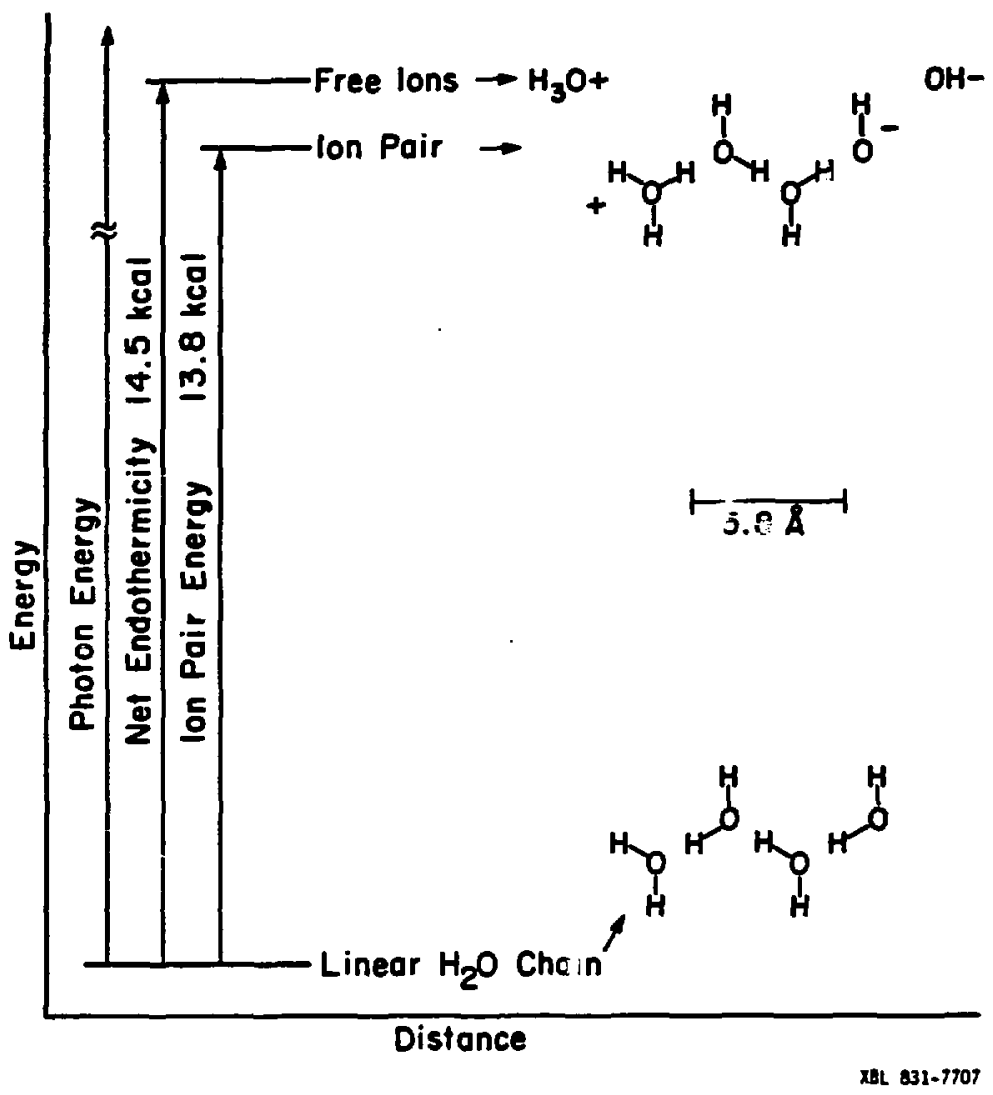


From Hong and Noolandi, the survival probability at time $t$ giv , initial ion pair formation at radius $r_{0}$ is

$$
P_{I S}(t)=\frac{U\left(r_{0}\right)}{U(-)}\left[1+\frac{r_{C}}{U(-)(\pi D t)^{1 / 2}}\right]
$$

where $r_{c}=e^{2} / \varepsilon k T$ and $D$ is the ionic diffusion coefficient. $U(r)$ is given by

$$
u(r)=e^{-\left(r_{c} / r\right)}+\left(\frac{D r_{c}}{\kappa a^{2}}-1\right) e^{-\left(r_{c} / a\right)}
$$

Where $a$ is the recombination radius and $k$ is the recombination velocity, At infinite time the survival probability becomes

$$
P_{I S}(\infty)=\frac{U\left(r_{0}\right)}{U(\infty)}
$$

From mic:oscopic reversibility, the ion pair formation distance for the thermal reastion, $r_{0}$, is identical to the recombination distance, a. Assuming the same formation distance for the laser reaction,

$$
\operatorname{PIS}(\infty)=\frac{D r_{c}}{\kappa a^{2}}\left[\frac{1}{e^{r_{c} / a}+\left(\left(D r_{c} / \kappa a^{2}\right)-1\right]}\right]
$$


This equation can be simplified by considering the first multiplicative term. Substituting $x=k_{-1} .{ }^{s}$ and $D=1 / 2 k_{\text {diff }} \ell^{2}, 36$

$$
\frac{D r_{c}}{x a^{2}}=\frac{k_{d i f f} \ell^{2} r c}{2 k_{-l a} l a^{2}}
$$

where $:$ is the average ionic jump distance during diffusion, $k_{-l a}$ is the recombination rate constant of the ion pair lsee equation (1) of the analysis section), and $k_{\text {diff }}$ is the rate constant for diffusive jumps. .

A much larger rumerator than denominator is obtained from the folloxing: from thermal recombination studies, the distance $r_{c}$ is a factor of 1.2 larger than $a=5.80$ \& and the $2.8 \&$ of $z$ is about two times smaller than a. Therefore, $\frac{r_{c} \ell^{2}}{a^{2} l}<1$. From thermal recombination studies, the recombination rate, $k_{-l a} \gg$ the diffusion rate, $k_{\text {diff. }}$ or,

$$
l \gg \frac{k_{d i f f} l^{2} r_{c}}{2 k_{-l a} 2 a^{2}}=\frac{D r_{c}}{k a^{2}}
$$

Equation 9 becomes

$$
\operatorname{PIS}(-)=\frac{D r_{c}}{k-1 a^{2 a^{2}}} \frac{1}{e^{I c^{/ a}}-1}
$$


The absolute magnitude of this equation cannot be determined because $k_{-1 a}$ is unkıown. However, the temperature and isotopic composition dejendence can be estimated. The thermal recombination rate studies already described show the recombination distance is approximately constant with temperature and isotopic composition, varying by less than 108 in $\mathrm{H}_{2} \mathrm{O}$ for a temperature change from $0^{\circ} \mathrm{C}$ to $45^{\circ} \mathrm{C}$ and by less than 158 at $25^{\circ} \mathrm{C}$ for an isotopic change from $\mathrm{B}_{2} \mathrm{O}$ to $\mathrm{D}_{2} \mathrm{O}$. The temperature dependence of $r_{c}=e^{2} / \varepsilon k T$ is a weak $\mathrm{T}^{-1}$ dependence, varying by about 158 from $0^{\circ} \mathrm{C}$ to $45^{\circ} \mathrm{C}$. Use of a constant $e^{r_{c} / a}$ and $a^{2}$ and replacing $D / k T$ with $\mu$ yields 35

$$
P_{I S}(-) \propto \mu / k_{-1 a}
$$

where $\mu$ is the ionic mobility. Thus, the probability of separation from the ion pair, PIS, is composed of $\mu$, which is dependent upon bulk properties of the solvent and $1 / \mathrm{k}-\mathrm{la}$, which is a local property of the solution. Now the full quantum yield expression can be grouped into a local and a bulk term. [See equations (1) and (2) of the analysis section for a definition of the rates.] Physically, $1 /\left(k_{1 a}+k_{2}\right)$ is the length of time a reactant remains activated, $k_{l a}$ is the rate of laser induced ion pair formation, $1 / k_{-1 a}$ is the length of time an ion pair remains before recombination, and $\mu$, the ionic mobility, is a measure of the ion pair separation efficiency. 


$$
P_{I P} P_{I S}=-\left[\frac{k_{1 a}}{k_{-1 a}\left(k_{1 a}+k_{2}\right)}\right] \quad[\mu]
$$

[local]

[bulk]

The remainder of this section examines each term of Equation 14 in more depth. Only the ionic mobility, $\mu$, is known quantitatively. Not enough information is known about vibrationally activated processes in a liquid to formulate a quantitative model for the other "local" terms. The goal is to obtain a qualitative idea how each term behaves as the temperature, excitation energy, and isotopic composition are changed.

2. $k_{1 a} / k_{-1 a}$

The energetics of the forward and reverse thermal reaction are known from thermal measurements. The barrier to recombination should be $\leq$ the $3.5 \mathrm{kcal} / \mathrm{mole}$ activation energy measured by an Arrhenius plot of the diffusion-controlled thermal recombination rate. Siras $\Delta H$ in $\mathrm{H}_{2} \mathrm{O}$ at $25^{\circ} \mathrm{C}$ is $13.5 \mathrm{kcal} / \mathrm{mole}$, the forward barrier is $\leq 17 \mathrm{kcal} / \mathrm{mole}$. Photons with enough energy to induce observable ionization ( $25.7 \mathrm{kcal} / \mathrm{mole}$ ) can produce activated reactants with $8.7 \mathrm{kcal} /$ mole of energy above the forward barfier or activated products with 8.7 $\mathrm{kcal} /$ mole above the reverse barrier.

Since the excitation energy is high and the reverse barrier is low, a local equilibrium is assumed. The 
model for $k_{l a} / k_{-l a}$ will be taken to be the density of states ratio of product to reactant, at the excitation energy. The semiclassical Marcus-Rice 37 expression is used for the density of states.

$$
N(E)=\frac{\left(E+E_{z}\right)^{s-1}}{(s-1) ! \prod_{i} h v_{i}}
$$

where $E$ is the total non-fixed energy, $s$ is the number of strongly-coupled modes sharing the energy, $h \nu_{i}$ is the energy per quantum of mode $i$ excitation and $E_{z}$ is the zero point energy. The non-fixed energy of the product is $E_{p h}+E_{z r}-E_{z p}-E_{0}$ (see Figure $v-6$ ). The non-fixed energy of the reactant is $\mathrm{E}_{\mathrm{ph}}$. The density of states ratio of product over reactant is then

$$
\frac{k_{1 a}}{k_{-1 a}}=\left(\frac{E_{p h}-\Delta E_{0}+E_{2 r}}{E_{p h}+E_{z r}}\right)^{s-1} \frac{i^{i \nu_{r}}}{\prod_{i} v_{p}}
$$

where the subscript $r$ or $p$ represents the reactant or product, respectively. $E_{\mathrm{ph}}$ represents the photon energy, and $\Delta E_{O}$ is the energy difference from product to reactant potential well minima.

This expression is exact if the ion pair yield is dominated by the yield a picosecond or two following excitation and if the reactant and product ion pair are in equilibrium at the excitation energy. Equilibrium will be achieved if the forward and reverse reaction rates 
Figure $\mathrm{V}-6$

One-dimensional potential energy surface for the water ionization reaction. The solid line shows the surface for the thermal reaction. The dotted potential surface is described near the end of section $c 3$ of the discussion. This potential is for the laser reaction when no strongly-coupled excited modes undergo a frequency change upon going from reactants to products. E for this potential is approximately equal to $\Delta H$, the thermal reaction endothermicity. 


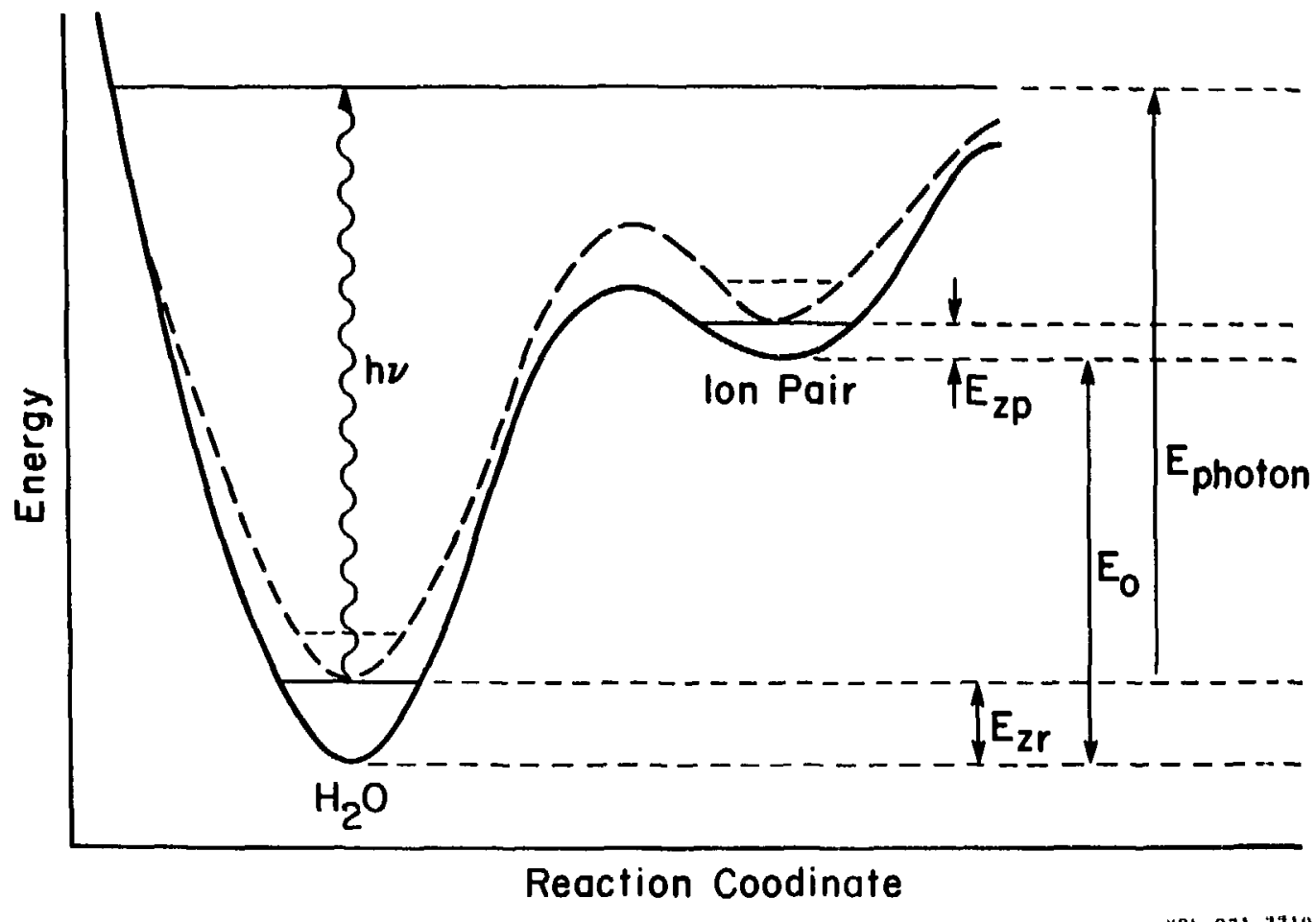

XBL 831-7718 
are rapid compared to the relaxation rate. In the opposite extreme where relaxation is rapid compared to the reaction rates, an RRKM type expression is more appropriate for the forward rate and the reverse rate is the thermal rate. An RRKM forward rate expression is nearly proportional to Equation (16) with the non-fixed energy for the transition state being shifted by the few kcal reverse barrier, $E_{b}$, and the power, s, increasina by one in the numerator.

$$
k_{l a}=\frac{\left(E_{p h}-E_{o}+E_{z r}-E_{b}\right)^{s}(s-1) ! \underset{i}{\pi h v_{r}}}{\left(E_{p h}+E_{z r}\right)^{s-1} s ! \underset{i}{\pi h} v_{T S}}
$$

This expression will have the same qualitative dependence on isotopic composition, and photon energy, as equation (16). The subscript, TS, represents the transition state,

3. $1 /\left(k_{l a}+k_{2}\right)$

The remaining term in the guantum yield contains the relaxation rate of activated reactants, $1 /\left(k_{1 a}+k_{2}\right)$. In the limit of large vibrational relaxation rate, $k_{2}$, this term becomes $1 / k_{2}$. No direct measurement of the vibrational relaxation rate of excited $O-H$ stretch motions in Iiquid water is available. However, relaxation measurements performed on several hydrocarbons 
and chlorinated hydrocarbons support some general conclusions about how vibrationally excited molecules relax in a room-temperature liquid.38-42 Both solvents interacting and solvents not interacting with the solute have been studied. $\mathrm{H}_{2} \mathrm{O}$ excited in $\mathrm{H}_{2} \mathrm{O}$, or $\mathrm{D}_{2} \mathrm{O}$ excited in $\mathrm{D}_{2} \mathrm{O}$ are expected to be more strongly interacting than HOD in $\mathrm{D}_{2} \mathrm{O}$.

In non-interacting solvents, results are explained by rapid intramolecular equilibration of energy amo.. levels energetically near and coupled to the excited level, followed by slower transfer to the solvent. For $\mathrm{CH}$ stretch excitation, other $\mathrm{CH}$ stretch modes and Fermi resonant bend overtones can couple efficiently with the excited $\mathrm{CH}$ stretch. In an interacting solvent, intermolecular relaxation to neighboring molecules can compete with intramolecular relaxation.

In water, Fermi resonant bends and intermolecular transfer are expected to influence the OH stretch relaxation. Figure $\mathrm{V}-7$ shows an energy level diagram for liquid $\mathrm{H}_{2} \mathrm{O}$ and liquid $\mathrm{D}_{2} \mathrm{O}$. Fermi resonant bend overtones are expected to contribute to relaxation of $\mathrm{OH}$ or OD stretch excitation in $\mathrm{H}_{2} \mathrm{O}$ or $\mathrm{D}_{2} \mathrm{O}$. Nearly resonant intermolecular transfer may be important for pure $\mathrm{H}_{2} \mathrm{O}$ or pure $D_{2} O$ because broad absorption lines inaintain near resonance by compensating for the anharmonicity of the overtone $v+v-1$ transition. Intermolecular transfer should te reduced for excitation of an $\mathrm{OH}$ stretch in predominantly $D_{2}$ solution where neighboring 
Figure V-7

Energy level diagram for $\mathrm{H}_{2} \mathrm{O}, \mathrm{HDO}$, and $\mathrm{D}_{2} \mathrm{O}$. Center frequencies are shown. Typical real widths are several hundred wavenumbers. Energy levels shown use fundamental frequencies. The diagram is similar for excitation of an $\mathrm{O}-\mathrm{H}$ stretch overtone. Letters $\mathrm{s}, \mathrm{b}, \mathrm{b}+\mathrm{L}, \mathrm{L}$, and $\mathrm{T}$ correspond to stretch, bend, bend plus libration combination, libration, and hindered translational or phonon modes, respectively. The average librational energy is shown for greater than three quanta of libration of $\mathrm{H}_{2} \mathrm{O}$ and for greater than two quanta of libration for HOD and $\mathrm{D}_{2} \mathrm{O}$. 


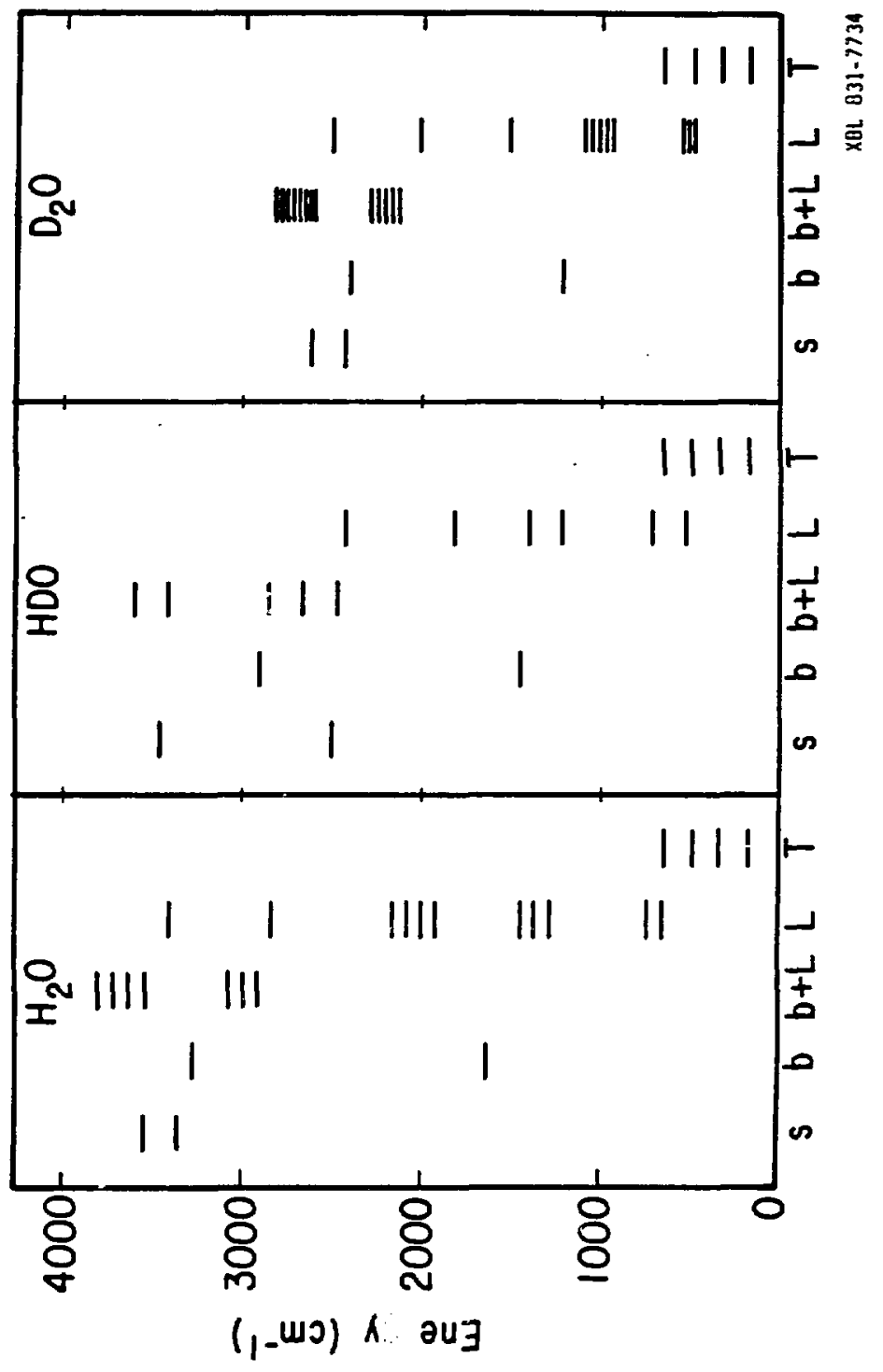


$\mathrm{D}_{2} \mathrm{O}$ molecules have no resonant transition. Spectroscopic studies show that at least for the fundamental, features in the $0-H$ stretching region of ice $I h$ and the amorphous solid are dominated by effects of intermolecular coupling of the oscillators43-46. A radical change in the importance of intermolecular coupling is not expected for the 1 iquid 43 .

c. Application of the quantum yield model to results.

The following section applies the quantum yield model developed in the previous section to the hydrogen mole fraction, wavelength, and temperature dependence of the quantum yield. The approach taken in each case is as follows:

(i) The ionic mobility is known as a function of mole fraction hydrogen and temperature. The effect this term has on the quantum yield can be removed from the results.

(ii) The form of the $k_{1 a} / k_{-1 a}$ term and therefore the shape of $k_{1 a} / k_{-l a}$ versus hyzrogen mole fraction, temperature and excitation wavelength can be obtained from equation (16).

(iii) Some energy relaxation pathways that may be important were mentioned. However, the influence which the relaxation 
pathway has upon the quantum yield is not known.

1. vs. $x_{H}$

Now we return to the question of why the quantum yield for HDO excitation in predominantly $\mathrm{D}_{2} \mathrm{O}$ solution is less than the quantum yield for $\mathrm{D}_{2} \mathrm{O}$. Each term which contributes to the quantum $y$ ield will be examined in turn to see if it can account for the non-monotonic dependence of the quantum yield on hydrogen mole fraction.

\section{(i) Ionic mobility}

The ionic mobility, $\mu$, as a function of hydrogen mole fraction is known quantitatively from the ionic conductivity of $\mathrm{H}^{+}$and $\mathrm{OH}^{-}$in isotopically mixed water. It increases monotonically with hydrogen mole fraction and therefore cannot account for the quantum yield dip for HOD in $D_{2} O$. The contribution of the mobility term to the guantum yield is removed and shown in Figure $v-8$.

\section{(ii) $\mathrm{k}_{1 \mathrm{a}} / \mathrm{k}-\mathrm{la}$}

Consider the $\mathrm{k}_{1 \mathrm{a}} / \mathrm{k}_{-1 \mathrm{a}}$ contribution to the quantum yield ratio $\phi\left(\mathrm{X}_{\mathrm{H}}\right) / \phi\left(D_{2} O\right)$, where $\mathrm{X}_{\mathrm{H}}$ is the hycirogen mole fraction. From equation (16), 
Figure $\mathrm{V}-8$

Quantum yield divided by ionic mobility. The points represent $\left(k_{1 a} / k_{-1 a}\right)\left[1 /\left(k_{1 a}+k_{2}\right)\right]$. 


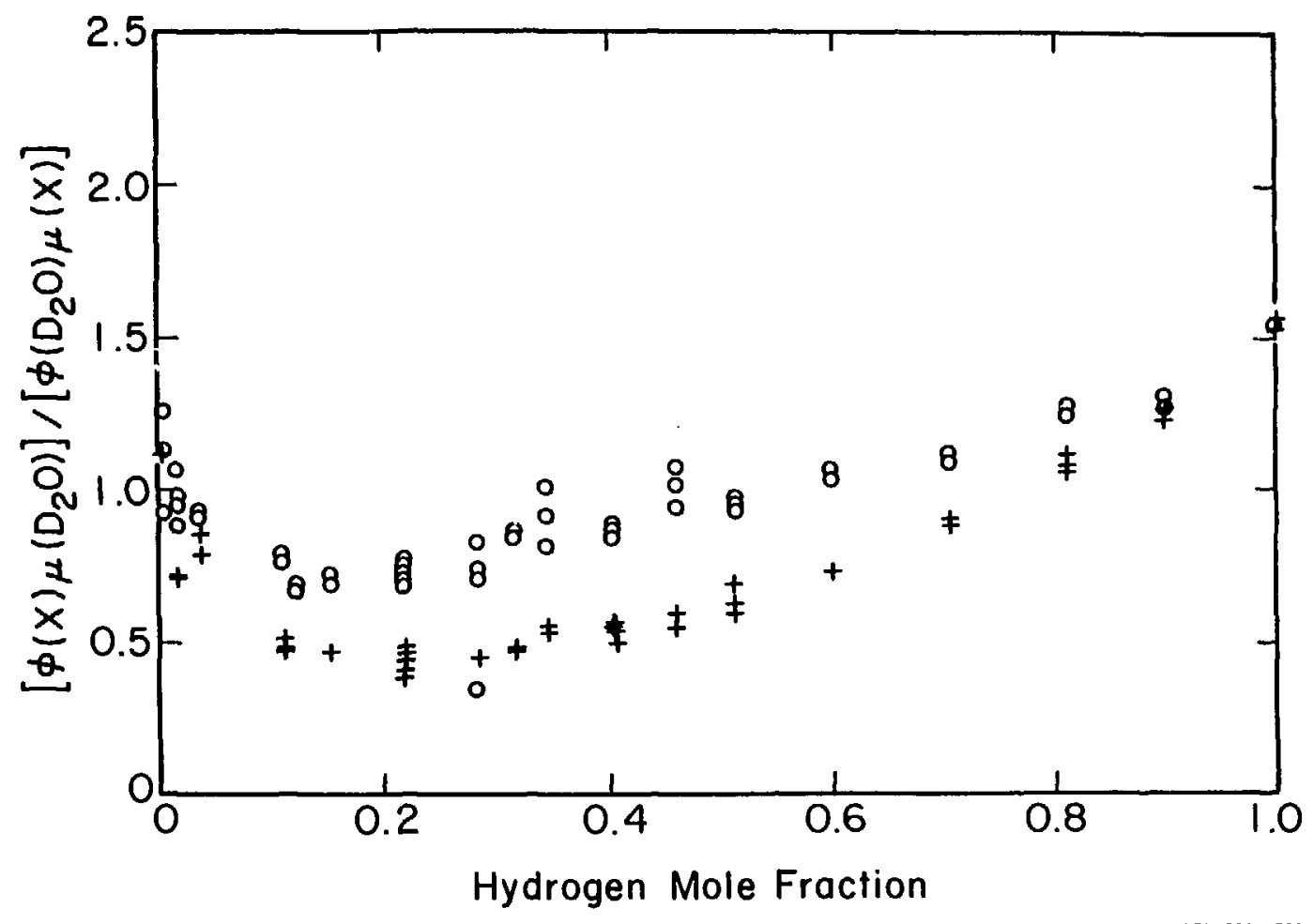




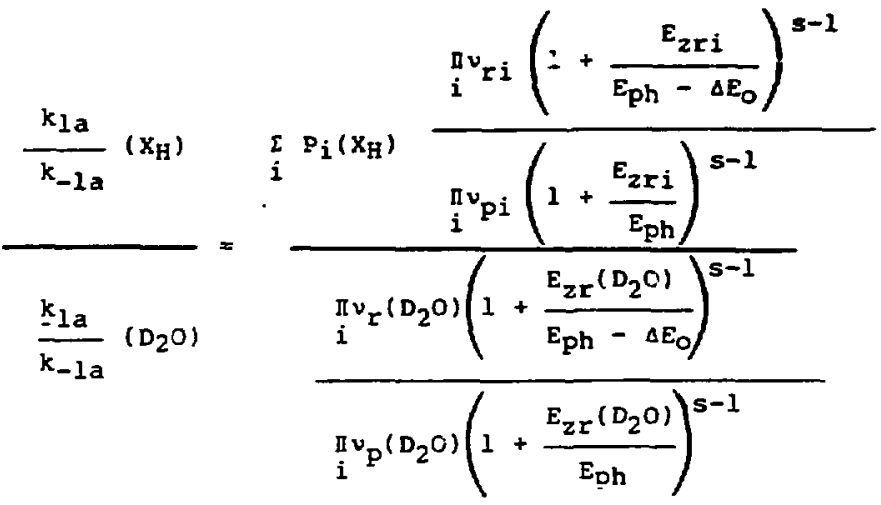

Index, $i$, is a labe' for each possible isotopica'ly substituted reactant, and $P_{i}\left(X_{H}\right)$ is the probability of species $i$ in a mixture with a hydrogen mole fraction of $\mathrm{x}_{\mathrm{H}}$. Rearranging the term involving frequencies, and using the Teller-Redich product rule 47 ,

$$
\frac{\pi v_{r}\left(x_{H}\right)}{\pi v_{r}\left(D_{2} O\right) \quad \pi v_{p}\left(\Sigma_{2} O\right)}=1
$$

will eliminate all terms involving frequencies. Also, since the de-minator is a constant, $D$, with hydrogen mole fraction, equation (17) can be simplified

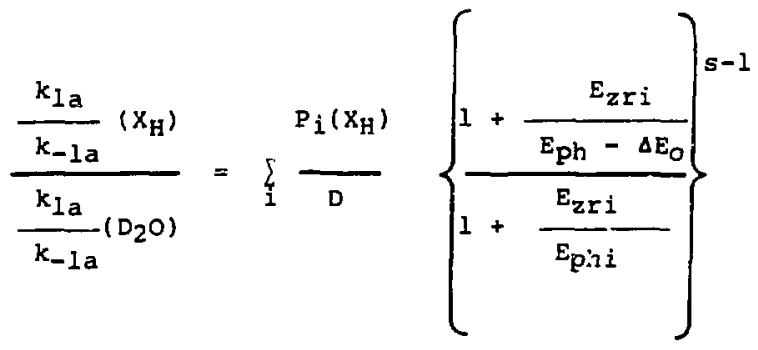


As the hydrogen mole fraction of a mixture increases, sfecies with more hydrogens substituted for deuterium become increasingly probable and have a greater relative contribution to equation (18b). A higher zero point energy for these species implies a monotonic increase of equation ( $18 \mathrm{~b}$ ) with hydrogen mole fraction.

$$
\text { (iii) } 1 /\left(k_{1 a}+k_{2}\right)
$$

The final term, the relaxation, must account for the non-monotonic dependence of $\phi$ with $x_{\mathrm{H}}$. For the discussion, it is helpful to divide the vibrational modes of the system into reactive and unreactive modes. Then excitation of HOD in $\mathrm{D}_{2} \mathrm{O}$ changes the energy flow relative to excitation of $\mathrm{H}_{2} \mathrm{C}$ in $\mathrm{H}_{2} \mathrm{O}$ or excitation of $\mathrm{D}_{2} \mathrm{O}$ in $\mathrm{D}_{2} \mathrm{O}$ by either (a) increasing the transfer rate to unreactive modes or (b) decreasing the transfer rate to reactive modes. Either the presence of an $O D$ in a molecule with excited $O H$ or presence of $O D$ (lack of $\mathrm{OH}$ ) bonds in the surrounding molecules causes the change of energy flow. This section demonstrates that the experimental data cannot distinguish between the two possible causes of change in energy flow. Nearly any model which has (1) a lower $\$$ for HDO excitation than for $\mathrm{H}_{2} \mathrm{O}$ or $\mathrm{D}_{2} \mathrm{O}$ excitation or (2) a lower $\phi$ for $\mathrm{OH}$ excitation in the presence of surrounding $O D$ bonds is in qualitative agreenent with the data. 
Curve $A$ of Figure $V-g$ shows a fit to the experimental data for excitation at $9400 \mathrm{~cm}^{-1}$. The dependence of upon ionic mobility is removed. The form for the quantum yield is the same as Equation (4). This corresponds to a lower quantum yield for HDC excitation, condition ( 1 ) above. Compared to $D_{2} C$ one potential difference in intramolecular energy flow is that transfer of energy from the excited $O H$ to the $O D$ bond of an HOD molecule should be slower than the nearly resonant transfer from an excited $O D$ to the other $O D$ of a $\mathrm{D}_{2} \mathrm{O}$ nolecule. If excitation of both oL bonds of an $\mathrm{L}_{2} \mathrm{O}$ molec 1 le promotes reaction, then the quantum yielc for HOD compared to $\mathrm{D}_{2} \mathrm{O}$ or $\mathrm{H}_{2} \mathrm{O}$ will be reduced.

Curve $A$ qualitatively fits the data, but as in the previous fit of Equation (4) to the quantum yield, there is insufficient upward curvature in the high hydrogen mole fraction region. A better fit is obtained with a model having a greater dependence of guantum yield on the fraction of neighboring bonds with the same isotopic identity as the excited bond. Figures $V-9$ and $V-10$, curves $B, C, D$, and $E$ show results from such a model. The model assumes a form for the quantum yield.

$$
\frac{\varphi\left(x_{H}\right)}{\mu}=\sum_{i j} \frac{\phi_{i j}}{\mu} \quad \varepsilon_{i} c_{i}\left(x_{H}\right) \& P_{j}\left(x_{H} / \sum_{i} \quad \varepsilon_{i} c_{i}\left(x_{H}\right) l\right.
$$


Figure $V-9$

Empirical curve ( - ) for three different empirical fits of $\phi / \mu$ and experimental points $(t)$ for $9400 \mathrm{~cm}^{-1}$ excitation at $25 \pm 1{ }^{\circ} \mathrm{C}$. The curves are described in the text. The line (--) represents contribution from $\mathrm{H}_{2} \mathrm{O}$ excitation, (•-• $\rightarrow$ from $\mathrm{HOD}$ excitation, and $(--)$ from $\mathrm{D}_{2} \mathrm{O}$ excitation. 


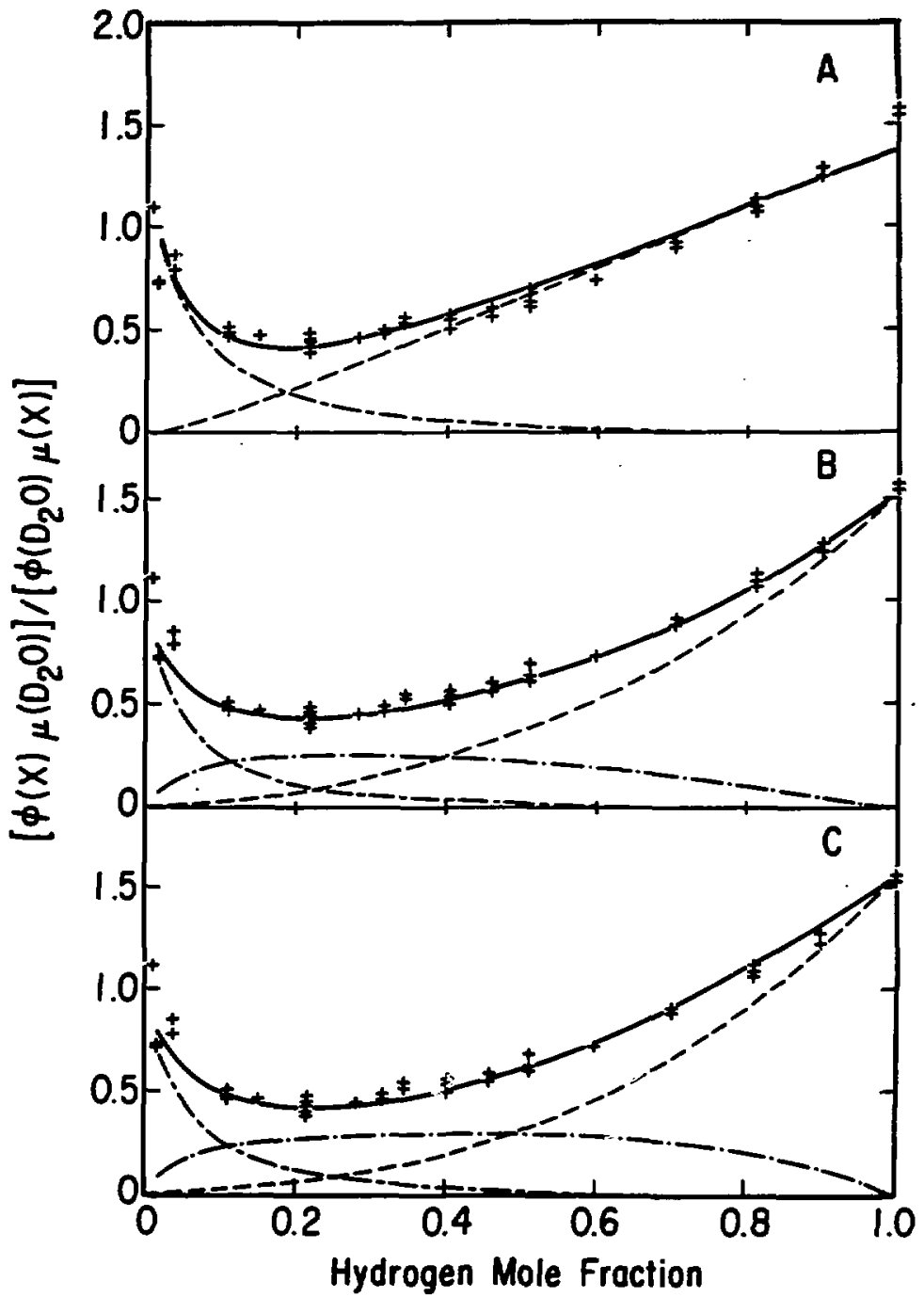

XEL B31-7711 
Figure $\mathrm{V}-10$

Empirical curve $(-)$ for three different empirical fits of $/ / u$, and experimental points $(+)$ for $9400 \mathrm{~cm}^{-1}$ excitation at $25 \pm 1^{\circ} \mathrm{C}$. The curves are described in the text. The line (--) represents contribution from. $\mathrm{H}_{2} \mathrm{O}$ excitation, (-- - from HOD excitation and (-- from $\mathrm{D}_{2} \mathrm{O}$ excitation. 


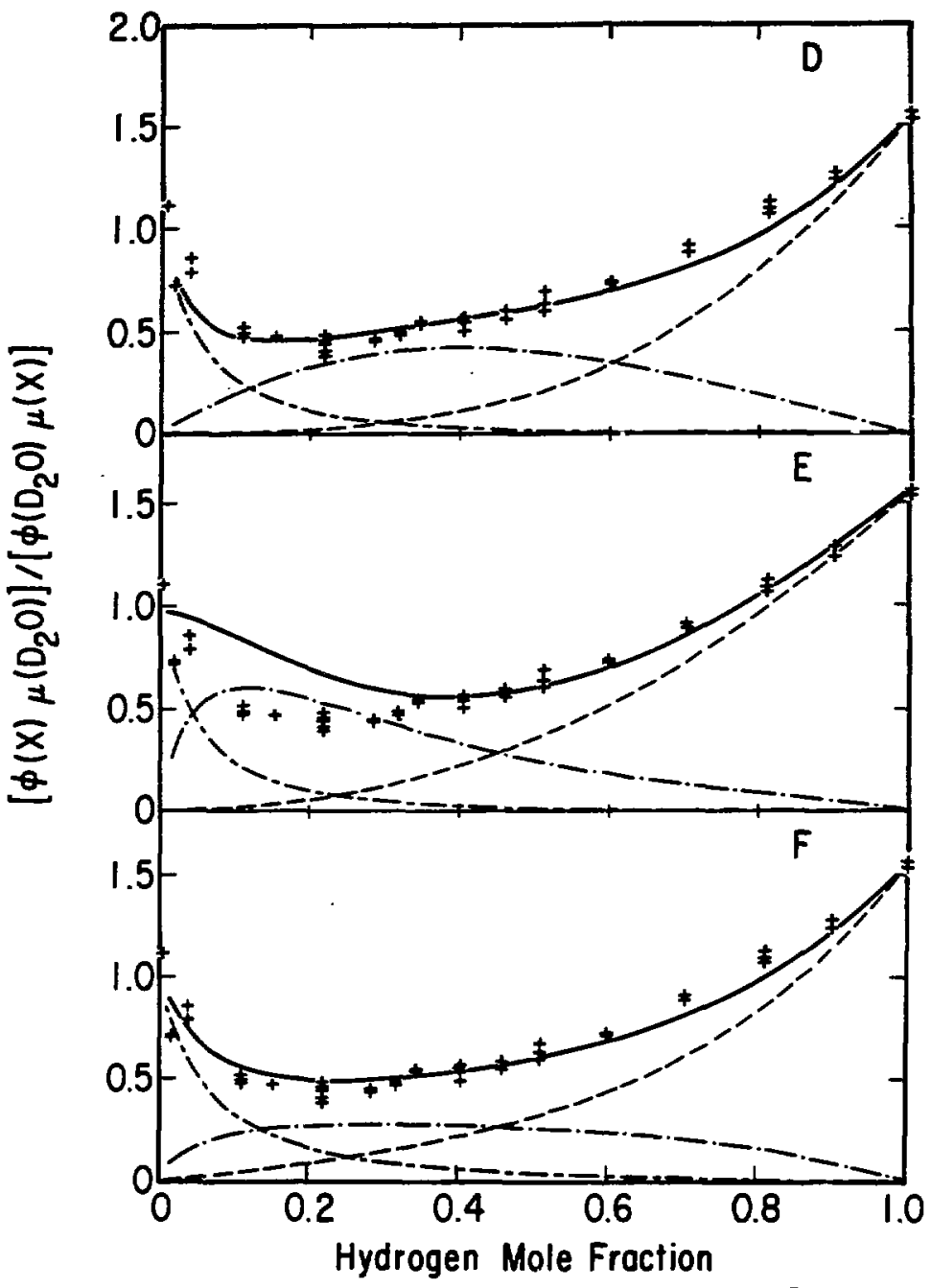

XBL $831-7712$ 
where $i$ represents an initially excited $\mathrm{H}_{2} \mathrm{O}, \mathrm{D}_{2} \mathrm{O}$, or HDO, $\varepsilon_{i} C_{i}\left(X_{H}\right) l$ is the absorbance of species $i$ in pathlength $l$ at concentration $C_{i}$ in a mixture of hydrogen mole fraction, $x_{H}$. Thus, species $i$ absorbs a photon with a probability $\varepsilon_{i} c_{i}\left(x_{H}\right) \ell / \Sigma_{i} \varepsilon_{i} c_{i}\left(x_{H}\right) l$. Index $j$ represents the isotopic configuration of two additional ol bonds, the possibilities being $\mathrm{OH}, \mathrm{OH}$; $\mathrm{OH}, \mathrm{OD}$; or $\mathrm{OD}, \mathrm{OD}$. The location of these bonds relative to the excited molecule is not specified. $P_{j}\left(X_{H}\right)$ is the probability of configuration $j$. Then $\phi / \mu$ in equation (19) represents the sum of the probabilities of excitation of configuration $i j$ times $\phi_{i j} / \mu$, the quantum yield/ionic mobility for excitation of species $i j$. The experimental points were fitted with four different sets of constraints on the coefficients $\phi_{i j} / \mu$. In each case, $\phi_{i j} / \mu$ for the entirely hydrogenated species is constrained to the experimental value in pure $\mathrm{H}_{2} \mathrm{O}$, and $\phi_{i j} / \mu$ for the entirely deuterated case is constrained to the experimental value in pure $\mathrm{D}_{2} \mathrm{O}$. The additional constraints on coefficients $\phi_{i j} / \mu$ that were tested and the possible physical interpretations are:

(1) $i_{i} / \mu$ for $i=$ HOD and $j=$ any possible isotopic configuration are approximately equal to each other but not to ${ }_{i j} / \mu$ for $i=H_{2} O, j=O H$, OH or to $i=D_{2} O, j=$ OD, OD. Other coefficients are not constrained. A possible interpretation of these constraints is if the presence of both $O H$ and $O D$ in the excited HOD molecules 
causes the quantum yield to be independent of the isotopic structure of the surrounding liquid, whereas with $\mathrm{H}_{2} \mathrm{O}$ or $\mathrm{D}_{2} \mathrm{O}$ excitation, the quantum yield depends on the surrounding concentration of bonds with the same isotope as that excited. Curve B of Figure V-9 shows close agreenent of this model with experimental results.

(2) $\phi_{i j} / \mu$ for $i=$ HOD and $j=O H$, OH is approximately equal to $\phi_{i j} / \mu$ for the pure solvents, $i=\mathrm{H}_{2} \mathrm{O}$ and $\mathrm{j}=\mathrm{OH}$, OH or $i=D_{2} O$ and $j=O D$, OD. A possible physical interpretation of this constraint is that the quanta of the excited ol bond must hop to two other ol bonds of the same isotopic species to produce a laser reaction. Then coefficients ${ }_{i j} / \mu$ will be large for $i=\mathrm{H}_{2} \mathrm{O}, j=\mathrm{OB}$, OB; $i=D_{2} O, j=O D, O D$, and, since the OH bond absorbs most of the light in an HOD molecule, $\phi_{i j} / \mu$ will be large for $i=H O D, j=O H, O H$. The model does not specify the location of the two or bonds represented by index $j$. They could be ol bonds of a neighboring molecule or of a linear hydrogen bonded chain. Curve C of Figure v-9 shows close agreement of this model with experimental results. (3) $\phi_{i j} / \mu$ fOr $i=$ HOD and $j=O H, O D$ is approximately equal to $\phi_{i j} / \mu$ for $i=H_{2} \mathrm{O}$ and $j=O H$, OH or for $i=D_{2} O$ and $j=O D, O D$. A possible physical interpretation of this constraint is that an excited molecule must have one particular adjoining molecule of the same isotopic identity to produce a laser reaction. For this model, $j$ denotes the isotopic identity of the molecule. Curve $D$ 
of Figure $V-9$ shows a less acceptable fit than models (1) and (2).

(4) $i j / \mu$ fOr $i=H O D$ and $j=O D, O D$ is approximately equal to $\phi_{i j} / \mu$ for $i=D_{2} O$ and $j=O D, O D$. If the exothermic intramolecular relaxation from the excited $\mathrm{OH}$ sicretch to the OD stretch of an HOD molecule dominates the initial relaxation, then the system might behave similarly to when $D_{2} O$ is the initially excited molecule. Curve $E$ of Figure $V-10$ shows a less acceptable fit than nodels (1) and (2).

Final coefficients for each model are shown in Table $v-1$.

A model in which nearby OD quenches activated OH was also tested to see if it could fit the non-monotonic dependence of $\phi$ on $x_{H}$. Curve $F$ of Figure V-10 shows a fit of

$$
\frac{\phi}{\mu}=\frac{\varepsilon_{O D} C_{O D}\left(x_{H}\right) \varepsilon \phi_{D} O+\sum_{K} P_{K} \frac{1}{A\left(1+n_{K} B\right)} E_{O H} C_{O H}\left(x_{H}\right) \& \phi_{H} O}{\varepsilon_{O D} C_{O D}\left(x_{H}\right) \ell+\varepsilon_{O H} C_{O H}\left(x_{H}\right) \ell}
$$

to the experimental data. The model corresponds to a relaxation rate, $k_{2}$, for excited $O H$ equal to $A+B n_{K} A$. This is the pure $\mathrm{H}_{2} \mathrm{O}$ rate, $A$, plus a term proportional to the number of nearest neighbor $O D$ bonds, $n_{K}$, in the excited molecule and in the six or bonds in the nearest neighbor sphere of the excited molecule. The quantum 
Table V-1

Coefficients, $\phi_{\mathrm{ij}} /\left(\lambda_{\mathrm{H}^{+}}+\lambda_{\mathrm{OH}^{-}}\right)^{a}$, for empirical $\phi / \mu$ vs. $x_{\mathrm{H}}$ curves.

coefficient $\left(\times 10^{10}\right)$ mol ohm $\mathrm{cm}^{-2}$

$\mathrm{H}_{2} \mathrm{O}(\mathrm{i}) \quad \mathrm{HDO}(\mathrm{i})$

curve $\mathrm{OH}, \mathrm{OH}(j) \mathrm{OH}, \mathrm{OD}(j), \mathrm{OD}, \mathrm{OD}(j) \mathrm{OH}, \mathrm{OH}(j) \mathrm{OH}, \mathrm{OD}(j)$

\begin{tabular}{|c|c|c|c|c|c|}
\hline B & 8. 28 & 3.2 & 1.95 & 3.36 & 2.48 \\
\hline$c$ & 8.28 & 2.06 & 1.14 & 7.07 & 2.66 \\
\hline D & 8.28 & 0.3 & 0.42 & 4.0 & 5.97 \\
\hline \multirow[t]{3}{*}{$\mathbf{E}$} & 8.28 & 3.5 & 0.274 & 3.0 & 0.667 \\
\hline & $H D O(i)$ & & & $D_{2} O(i)$ & \\
\hline & $O D, O D(j)$ & & $\mathrm{OH}, \mathrm{OH}(\mathrm{j})$ & $O H, O D(j)$ & $O D, O D(j)$ \\
\hline B & $\underline{1.56}$ & & 0.82 & 2.18 & 5.06 \\
\hline$c$ & 1.82 & & 1.11 & 2.21 & 5.06 \\
\hline$D$ & 0.748 & & 1.8 & 2.05 & 5.06 \\
\hline E & 6.0 & & 0.667 & 0.2 & 5.06 \\
\hline
\end{tabular}

a

${ }_{i j} /\left(\lambda_{\mathrm{H}^{+}}+\lambda \mathrm{OH}^{-}\right)$is proportional to $/ \mu . \quad \lambda=F(\mu)$ where $F$ is Faraday's constant. Index $i$ denotes the initially excited species. Index $j$ represents the isotopic identity of two additional oc bonds. 
yield for $D_{2} O$ excitation is independent of the isotopic composition of the nearest neighbor oL bonds. $B$ is a proportionality constant. $P_{K}$ is the probability of configuration $k$. A satisfactory fit of the experimental data is obtained with $B=0.55$. Thus, when two of the seven $O-L$ bonds nearest to an excited $O-H$ are $O-D$ bonds, the relaxation is 2.1 times as fast as for pure $\mathrm{H}_{2} \mathrm{O}$. The success of model (1) in fitting the experimental data suggests a model involving resonant transfers of $\mathrm{OH}$ stretch excitation may be involved in the laser reaction. A physically reasonable model requiring resonant transfer of oH stretch quanta in a linear hydrogen-bonded chain of four water molecules can be devised. The ion pair intermediate of the model to be described is identical to that postulated for the thermal reaction. Motion of the three hydrogen-bonded hydrogens of a four-molecule linear chain intermediate is required to form an ion pair. The three molecules could be activated by a minimum of two hops of ol stretch quanta from the excited molecule to neighboring molecules. Assume atom nine of molecule $C$ of Figure $\mathrm{V}-11$ is excited. A hop of one quantum to a molecule above $c$ along with the hop of a second quantum to one molecule below $C$ will form an "activated" three-molecule linear chain.

The model has no adjustable paramecers, other than that the results are constrained to pass through experimental points for pure $\mathrm{H}_{2} \mathrm{O}$ and pure $\mathrm{D}_{2} \mathrm{O}$. The following assumptions are made: 
Figure V-11

A tetrahedral cluster of hyārogen-bonded water molecules is shown. 


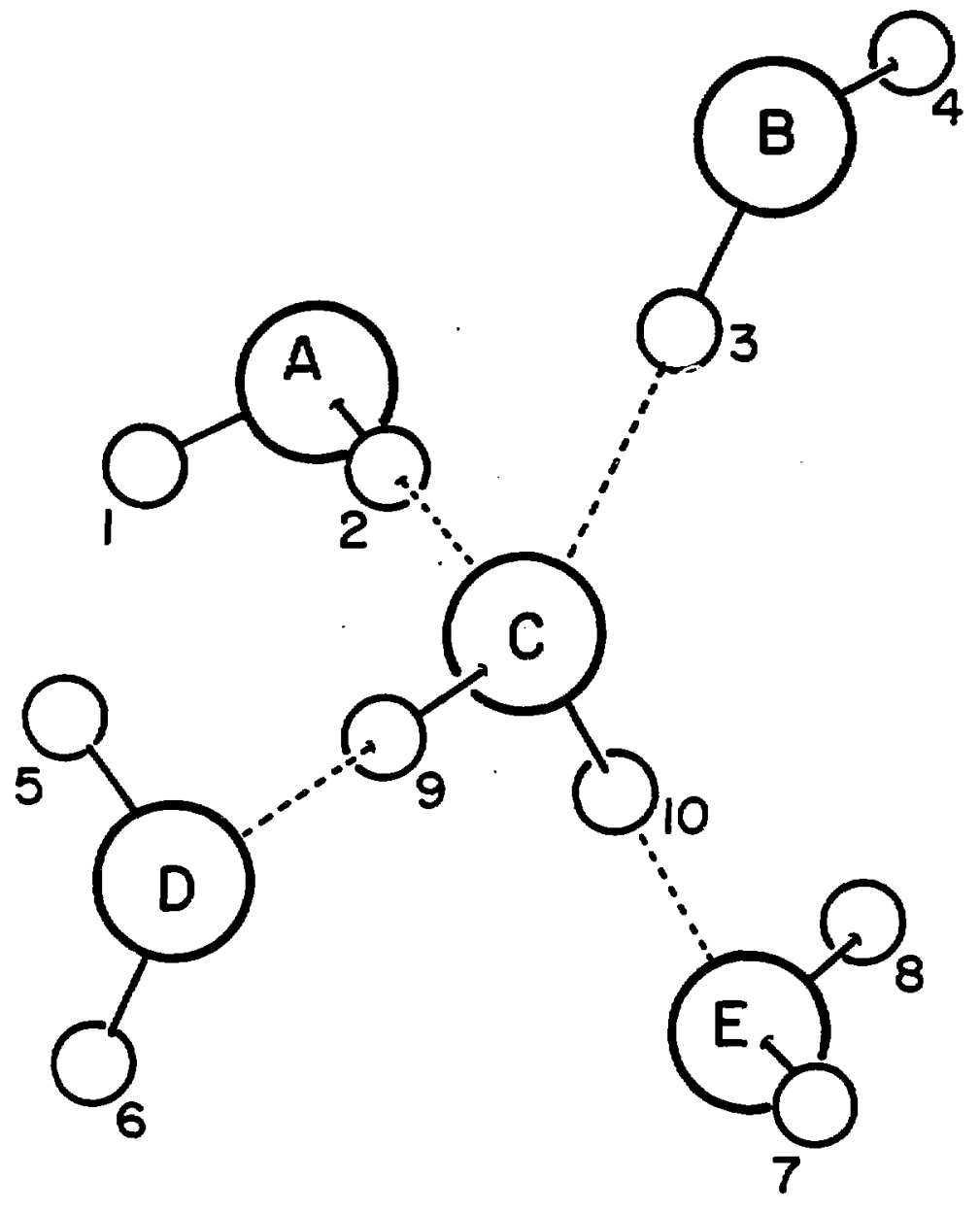

XBL 831-7704 
(A) In order for reaction to occur, at least one quantum must be in each of three molecules in a linear chain. In other words, in molecules $A, C$, and $E ; A, C$, and $D ; B, C$, and $E$; or $B, C$, and $D$.

(B) An excited $\mathrm{H}_{2} \mathrm{O}$ or an excited $\mathrm{D}_{2} \mathrm{O}$ shares excitation among both bonds in the molecule. An excited HOD does not. This means that if an OH in HOD with the hydrogen represented by atom 9 in Figure $V-11$ is excited, then quanta cannot hop to molecule $E$ because atom 10 is a deuterium.

(C) The rate of ol stretch transfer, khop, from $\mathrm{H}_{2} \mathrm{O}^{*}$ or $\mathrm{D}_{2} \mathrm{O}^{*}$ to bonds $2,3,5,6,7$, and 8 is proportional to the number with the same isotope as the excited oL stretch. The * represents the initially excited molecule.

(D) Since HOD* does not share excitation between the $O D$ and $O H$, the rate of transfer from HOD* is proportional to the number of bonds, 2, 3, 5, and 6 with isotopes identical to the excited bond.

The dependence of $\phi$ on the rate of energy transfer out of the initially excited bond depends on the relative rate of thermalization. If thermalization is fast compared to the hop rate, then the two hops necessary to activate the three-molecule chain before thermalization occur with probability proportional to $\left(k^{i}{ }_{h o p}\right)^{2} P_{i 2} \cdot P_{i 2}$ is the probability that for isotopic configuration, $i$, two quanta that randomly jump will form an activated three-molecule linear chain. The rate $k^{i}$ hop is the 
hop rate for isotopic configuration, i. If the hop rate is fast compared to the relaxation rate, then is independent of the hopping rate and is proportional to $P_{i 2}$. Let the probability of isotopic configuration $i$ be $P_{\text {il }}\left(X_{H}\right)$. Let

No. of activated configurations for a hop of 2 quanta $P_{i 2}=$ Total number of configurations for 2 quanta

and

$$
\mathrm{k}_{\text {hop }}^{\mathrm{i}}=\mathrm{A} n_{\mathrm{H}}
$$

where $n_{B}$ is the number of hydrogens on bonds $2,3,5,6$, 7 , and 8 when an $\mathrm{H}_{2} \mathrm{O}$ is excited, or the number on 2, 3, 5, and 6 for $O H$ stretch excitation of an HOD molecule. A is a proportionality constant. Then the quantum yield for excitation of an $\mathrm{OH}$ or $\mathrm{OD}$ bond is

$$
\phi_{O D}\left(1-x_{H}\right)=\phi_{O H}\left(x_{H}\right) \propto \sum_{i}^{\sum} P_{i l}\left(k_{h O p}^{i}\right)^{2} P_{i 2}
$$

if the hop rate is slow compared to the relaxation rate or

$$
\phi_{O D}\left(1-X_{H}\right)=\phi_{O H}\left(X_{H}\right) \propto \sum_{i} P_{i 1} P_{i 2}
$$

if the hop rate is fast compared to the relaxation rate. The proportionality constants for OH or OD excitation are found by constraining values in pure $\mathrm{H}_{2} \mathrm{O}$ and $\mathrm{D}_{2} \mathrm{O}$ to the experimental result.

In addition, two other conditions were tested for the model providing the best fit of the data [Equation (23)]. 
(1) That if the quanta must remain in the OB bonds in the linear chain for reaction to occur? for instance. with molecules $A, C$, and $D$ activated, if atom 1 or atom 10 of Figure $\mathrm{V}-11$ is the same isotope as the initially excited bond, then reaction occurs with one-half the probability that it does when the atom is the opposite isotope. The reason is that if either of these atoms is the same isotope as that initially excited, then a guanturn of stretch is shared equally between the two identical molecular $\mathrm{O}-\mathrm{L}$ bonds and only one of these bonds is in the activated linear chain.

(2) What if the guanta need not remain in the linear bonded $\mathrm{OH}$ bonds but can be in any mode of the linearly bonded molecules?

Figure $V-12$, curves $G$ and $H$ show the results for excitation at $9400 \mathrm{~cm}^{-1}$ (three $\mathrm{OH}$ stretch or four OD) assuming an activated three-molesule chain is formed with probability $\left(k^{i}{ }_{h o p}\right)^{2} P_{i}$. This is the model where thermalization competes effectively with the OH stretch hop. Curve $G$ assumes the guanta must remain in the $\mathrm{OH}$ bonds of the linear chain, and curve $H$ assumes that the energy need not remain in the OH stretch. The best fit is for curve $H$. The shape of the curve is correct, with the minimum at approximately the correci mole fraction, but the minimum is lower than the experimental points. The discrepancy is not severe for a model with no adjustable parameters. A greater quantum yield for HOD 
excitation relative to $\mathrm{H}_{2} \mathrm{O}$ or $\mathrm{D}_{2} \mathrm{O}$ would produce a better fit. This could arise from several sources which the model does not include:

(i) There could be a contribution from a thermal component, not requiring resonant transfer, which is approximately constant with hydrogen mole fraction.

(ii) A possible contribution from the next sphere of hydrogen-bonded water molecules was not included. Second sphere reactions favor $\mathrm{H}_{2} \mathrm{O}, \mathrm{D}_{2} \mathrm{O}$ mixtures relative to the pure isotope because in order to form an activated reactant involving a second nearest neighbor molecule, both quanta that hop must leave the excited molecule through the same nearest neighbor $\mathrm{r}_{2} \mathrm{O}$. If an excited $\mathrm{OH}$ is surrounded by other oH's, then there is a cone-third chance that the second quantum will leave the excited molecule by entering the same molecule the first quantum entered. If surrounded by only one $\mathrm{OH}$ and many other $O D$, the second quantum must leave through the single available $\mathrm{OH}$.

$$
\text { (iii) HOD may not relax as rapidly as } \mathrm{H}_{2} \mathrm{O} \text { or } \mathrm{D}_{2} \mathrm{O}
$$
because the bend overtone in HOD is not nearly as resonant with the $\mathrm{OH}$ stretch as is the case for $\mathrm{H}_{2} \mathrm{O}$ or $D_{2} O$. This would raise the quantum yield for HOD relative to $\mathrm{H}_{2} \mathrm{O}$ or $\mathrm{D}_{2} \mathrm{O}$ and would raise the empirical curve closer to the experimental points. 
The curve assuming a rapid hop rate compared to the thermalization rate, equation (24), with activation not constrained to the linearly bonded oH bonds, does not produce a satisfactory fit of the experimental data (Figure v-12b).

\section{2. vs. T}

If the contribution of the "bulk" mobility term is divided out of the quantum yield, the remainder, $\phi / \mu$ decreases with increasing temperature. In other words, - increases more slowly with $T$ than does $\mu$, the mobility. The $k_{l a} / k_{-l a}$ term of Equation (16) will increase with increasing temperature. Therefore, either (1) the relaxation rate increases with temperature, or (2) the ion pair separation occurs at an effective temperature higher than ambient or both. The photon energy is greater than the endothermicity for the ion pair formation reaction. The excess energy could raise the effective temperature seen by the diffusing ion above the ambient temperature. Ten kcal of excess energy distributed over 100 modes, about 16 molecules, increases the temperature by about $50^{\circ} \mathrm{C}$.

If the ions diffuse faster than the excess energy does, then the use of the ambient temperature is correct. If energy diffusion is faster than ionic diffusion, the excess photon energy can raise the temperature in 
Figure v-12

Empirical curve (-) and experimental points (+)

for $\phi / \mu$. Experimental points are for $9400 \mathrm{~cm}^{-1}$ excitation at $25 \pm 1^{\circ} \mathrm{C}$. Activation of the water ionization is by hopping of $\mathrm{OH}$ stretch quanta to neighboring molecules. The line (---) represents contribution from $\mathrm{H}_{2} \mathrm{O}$ excitation, (-*-) from HOD excitation, and (---) from $\mathrm{D}_{2} \mathrm{O}$ excitation. 


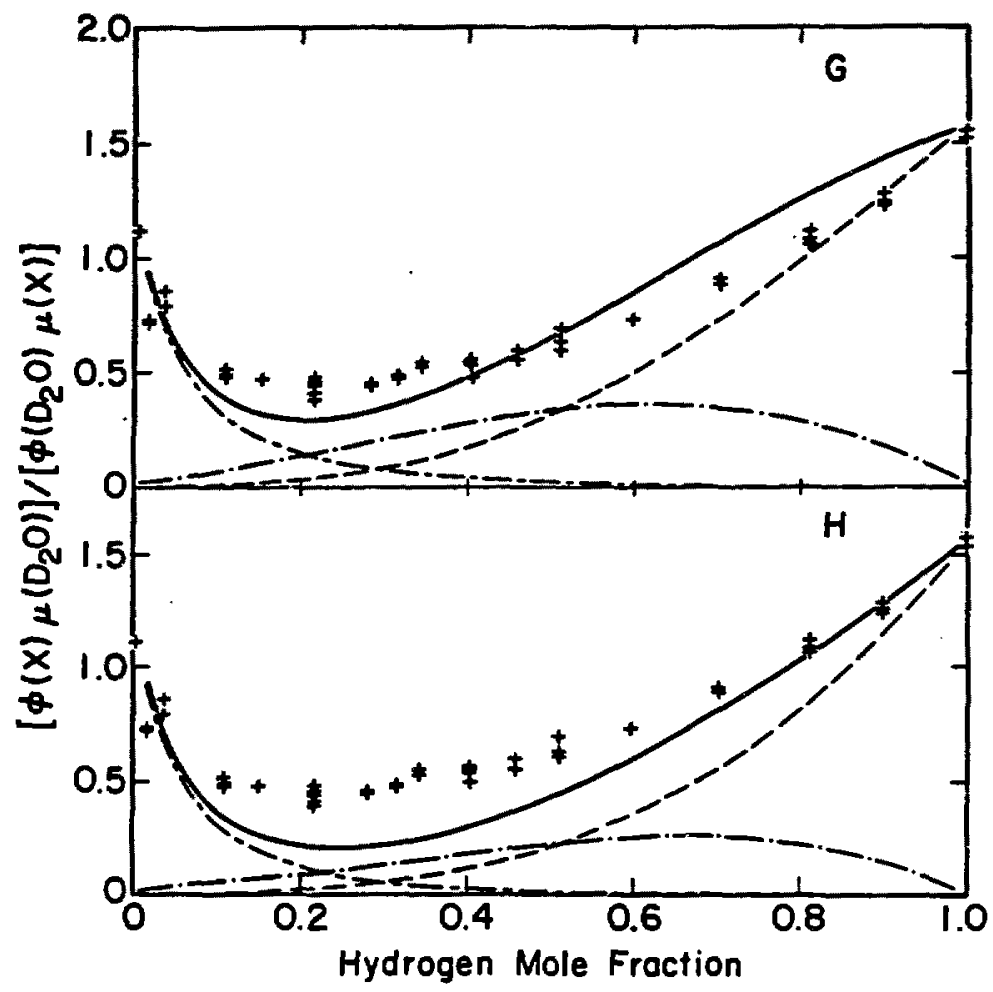

XBL ESt-7710 
Figure $\mathrm{V}-12 \mathrm{~b}$

Empirical curve (-) and experimental points $i+$ )

for $\$ / \mu$. Experimental points are for $9400 \mathrm{~cm}^{-1}$ excitation at $25 \pm 1^{\circ} \mathrm{C}$. Activation of the water ionization is by hopping of $\mathrm{OH}$ stretch quanta to neighboring molecules with a hop rate fast compared to the energy thermalization rate. The line (---) represents contribution from $\mathrm{H}_{2} \mathrm{O}$ excitation, (-- ) from HOD excitation, and (---) from $\mathrm{D}_{2} \mathrm{O}$ excitation. 


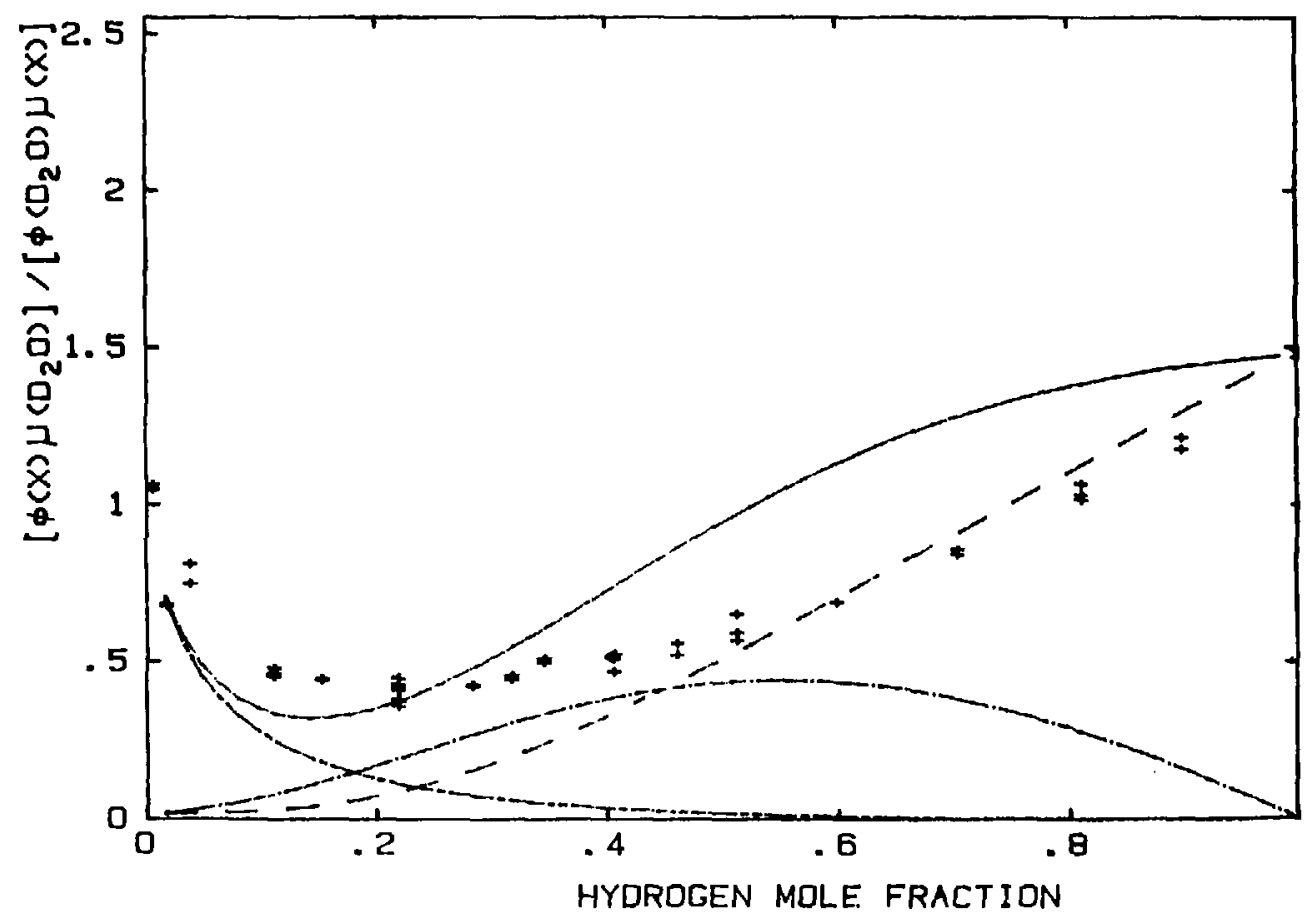


Figure V-13

The quantum yield divided by ionic mobility at a higher than ambient temperature as a function of temperature for excitation of liquid water near $v=3 \mathrm{OH}$ stretch $(\bullet,+)$ and near $v=4$ OH stretch $(x, \Delta, 0)$. The mobility used is for $14^{\circ} \mathrm{C}$ higher than ambient for excitation near $\mathrm{v}=3$ and is $60^{\circ} \mathrm{C}$ higher than ambient for excitation near $v=4$. 


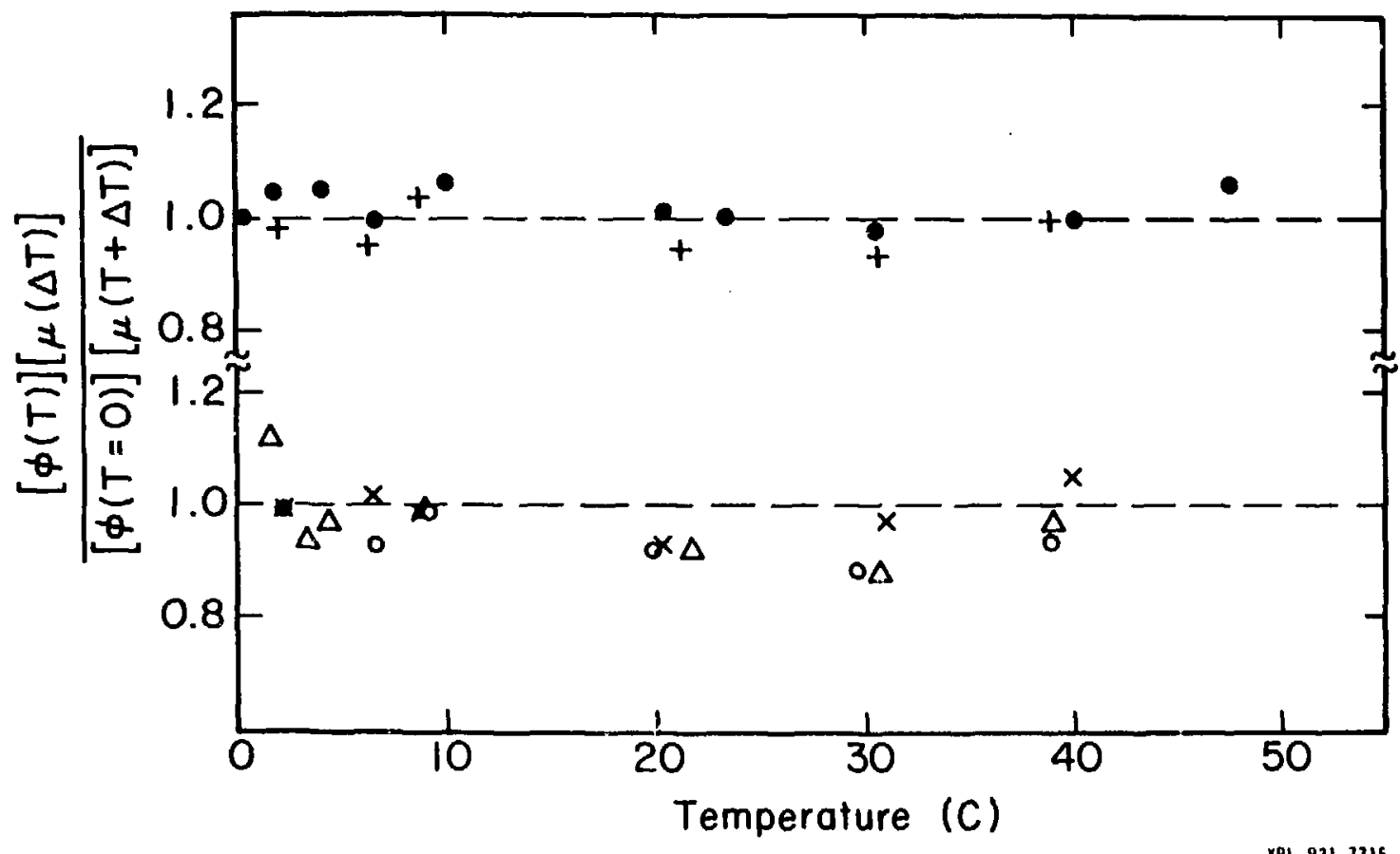


the neighborhood of the diffusing ions above ambient. A thermal conductivity for water of $6.4 \mathrm{MW} / \mathrm{cm} \mathrm{K} 48$ yields a thermal diffusivity of $1.5 \times 10^{-3} \mathrm{~cm}^{2} / \mathrm{sec}$, one order of magnitude greater than the $1.46 \times 10^{-4} \mathrm{~cm}^{2} / \mathrm{sec}$ diffusion coefficient for $\mathrm{H}^{+}$and $\mathrm{OH}^{-}$ions. Figure $\mathrm{V}-13$ shows that the temperatue dependence of the quantum yield could be attributed to ion pair separation at a temperature 14 degrees above ambient for excitation near $v=3$ of the $\mathrm{OH}$ stretch or at a temperature 60 degrees above ambient for excitation near $v=4$. More excess energy is available for excitation near $v=4$ than for excitation near $v=3$ (* $7900 \mathrm{~cm}^{-1}$ versus $5000 \mathrm{~cm}^{-1}$ ). Therefore, the effective temperature should be greater than for excitation near $\mathrm{v}=3$.

3. $\phi$ vs $\lambda$

The goal of this section is to characterize the experimental results. It is assumed that the $\phi$ vs $\lambda$ dependence is dominated by the $k_{1 a} / k_{-} l a$ term described by equation (16). The actual dependence of the relaxation term, $1 /\left(k_{1 a}+k_{2}\right)$, on excitation energy is not known but is assumed to be negligible. The mobility term does not contribute. The previous section showed the effective temperature of the ion pair separation could be affected by the amount of excess photon energy. However, a change in effective temperature from $24.0^{\circ} \mathrm{C}$ to $70.0^{\circ} \mathrm{C}$ produces 
less than a factor of two increrse in the ionic mobility compared to approximately two orders of magnitude increase in quantum yield over the $9400 \mathrm{~cm}^{-1}$ to $-12,625 \mathrm{~cm}^{-1}$ wavelength range reguired to produce the corresponding effective temperature.

Equation (16) should be considered an empirical equation. Fryatinn ( 16 ) is exact for a was nhace reaction where $E_{2 r}, \Delta E_{O}, v_{i}$, and $s$ have a well defined exact Fhysical definition. In a liquid, these parameters are not physically well defined. They are dependent upon an unknown number of coupled vibrational modes of undetermined frequency. The approach will be (1) to define $E_{z r}$, $\Delta E_{0}, v_{i}$, and $s$ for a hypothetical gas phase system, (2) to compare a gas phase system to a liquid phase system, and (3) to estimate reasonable relationships between $E_{z r}, \Delta E_{0}$, and $s$ for the liquid water system. Values are then inferred from the experimental data. $E_{z r}$ is the reactant zero point energy: $\Delta \mathrm{E}_{\mathrm{g}}$ is energy from reactant to product well bottom, and $s$ is the number of coupled modes. Thus, the equation must be considered empirical until experimental results from other systems confirm or deny any physical meaning for the parameters of the equation. Some concepts developed here may be applicable to unimolecular reactions of other large systems following non-statistical activation. 
Consider a unimolecular gas phase isomerization systen with two stable wells separated by a barrier. If a single molecule in the mixture is excited above the barrier, the fraction of excited population in each well is determined by the density of states ratio of Equation 16. The system is closed on the timescale of molecule collisions, with $E_{z r}$ and $s$ determined by the modes of the excited molecule. There are other modes present in the unexcited molecules of the system. These modes are not included in $E_{z r}$ or $s$ because they are not coupled to the excited molecule until a collision occurs.

Contrast this situation with the open system of the liquid water dissociative ionization reaction. The excited or bond is coupled not only to the intramolecular modes, the local phonon (hindered translational), and local librational (hindered rotational) modes of the excited molecule, but also to most other $\mathrm{H}_{2} \mathrm{O}$ molecules in the solution through an extensive network of strong hydrogen bonds. The number of modes, the mode frequencies, and the magnitude of $\Delta \mathrm{E}_{\mathrm{O}}$ is not obvious.

It is useful to break the system into two parts, one part being modes "strongly coupled" to the excited OH stretch, and one part being "weakly coupled". The system is then identical to the gas phase system in the Iimit of infinitesimal "weak coupling" and of "strong coupling" large enough to ensure energy randomization among strongly coupled modes at a rate rapid compared to 
Figure V-14

Experimental points for the quantum yield versus excitation wavelength $(0)$ along with several fits to $\phi=A\left(E_{p h}-E_{0}+E_{2 r} / E_{p h}+E_{z r}\right)^{s-1}$. The parameters used are $s=9, E_{z r}=1200 \mathrm{~cm}^{-1}, E_{o}=7041 \mathrm{~cm}^{-1}$, and $A=1.70 \times 10^{-3}(-) ; s=12, E_{z F}=1000 \mathrm{~cm}^{-1}$, $e_{0}=5900 \mathrm{~cm}^{-1}$, and $A=1.60 \times 10^{-3}(--) ;$ and $s=16$, $E_{z r}=650 \mathrm{~cm}^{-1}, E_{0}=4734 \mathrm{~cm}^{-1}$, and $A=3.89 \times 10^{-3}(\ldots \ldots)$. 


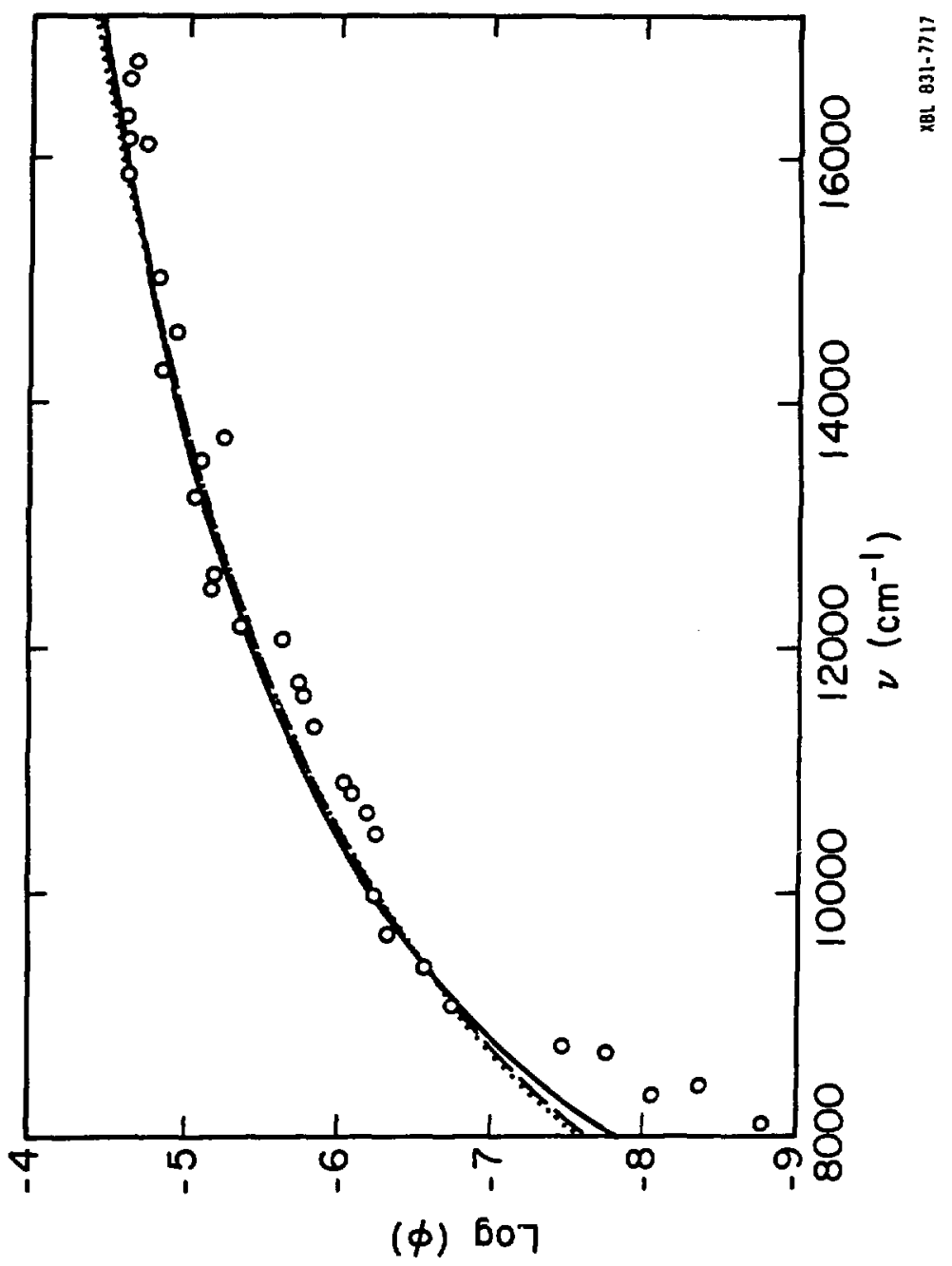


the reaction rate. The identity of the strongly coupled modes depends on the details of the energy flow. The modes physically close to the excited molecule or with fundamental or overtones close in energy to the excited bond are expected to be strongly coupled. Since details of the energy flow are not known, the approach taken is to fit a curve with the form of Equation (16) to the data. The larger $s$ is, the more modes involved. The larger $E_{z}$ is for a given $s$, the higher the average mode frequency. To determine $\Delta E_{O}$, the laser reaction is compared to the thermal reaction (see Figure $v-6$ ). For a thermal reaction, $\Delta E_{O}$ is estimated from the $\Delta H$ for the thermal reaction and the zero point energies of reactants and products. If it is assumed that the thermal vibrational energy of reactant is equal to the vibrational energy of products, then $\Delta H$ is the same as the energy between reactant and product zero point, and

$$
\Delta E_{O}=\Delta H+E_{z r}-E_{z P}
$$

The quantity $\Delta E_{O}$ is unambiguous. Every mode which has an energy change upon going from reactant ts product will contribute to $\Delta \mathrm{E}_{\mathrm{O}}$. If modes are included in calculation of the zero point energies which do not experience a frequency change upon going from reactant to product, then there is no contribution to $\Delta E_{0}$ because $E_{z r}-E_{z p}$ is zero. 
For the laser reaction, the modes which are coupled strongly are not necessarily the same modes which experience a frequency shift upon product formation. Only the modes strongly coupled to the excited bond should be included in the density of states calculation and should be included in calculation of $\Delta E_{O}$. Thus, $\Delta E_{0}$ can range between $\Delta E_{O}$ (thermal) to $\Delta H$ depending on how many modes with a frequency shift are strongly coupled to the excited on bond. If every mode with a frequency shift is strongly coupled to the excited $\mathrm{O}-\mathrm{H}$ bond, then $\Delta \mathrm{E}_{\mathrm{O}}($ laser $)=$ $\Delta E_{0}$ (thermal). If no modes with a frequency shift are strongly coupled, then $\Delta \mathrm{E}_{\mathrm{O}}($ laser $)=\Delta \mathrm{H}$.

The thermal value for $\Delta E_{0}$ is estimated from mode frequencies given by More $\mathrm{O}^{\prime}$ Ferrall et $\bar{c} \mathrm{l}$. for $\mathrm{H}_{2} \mathrm{O}$, $\mathrm{H}_{3} \mathrm{O}^{+} 49,50$ and $\mathrm{OH}^{-}\left(\mathrm{H}_{2} \mathrm{O}\right)_{3}^{51}$. The frequencies are calculated from a molecular force field with force constants adjusted to match known experimental frequencies for $\mathrm{H}_{2} \mathrm{O}, \mathrm{H}_{3} \mathrm{O}^{+} 52-57$, and $\mathrm{OH}^{-}\left(\mathrm{H}_{2} \mathrm{O}\right)_{3}^{58-63}$. Degeneracies and frequencies used in the zero point energy calcuiation are given in Table $\mathrm{V}-2$. In $\mathrm{H}_{2} \mathrm{O}$, the zero point energy for ionic products is $25,480 \mathrm{~cm}^{-1}$ and $27,787.5 \mathrm{~cm}^{-1}$ for the reactant water. $\Delta E_{0}$ (laser) can range between $\Delta H=4734 \mathrm{~cm}^{-1}$ and $\Delta E_{0}($ thermal $)=7041.5 \mathrm{~cm}^{-1}$. The irregular dependence of with excitation energy requires a fit of quantum yields with excitation at equivalent points in successive OH stretch bands. Quantum yields at $6700 \mathrm{~cm}^{-1}, 9983 \mathrm{~cm}^{-1}$. $13,240 \mathrm{~cm}^{-1}$, and $16,500 \mathrm{~cm}^{-1}$, corresponding to excitation 
Table $v-2$

Frequenciesa used in zero point energy calculation

\begin{tabular}{|c|c|c|c|}
\hline \multicolumn{2}{|c|}{$\mathrm{H}_{3} \mathrm{O}^{+}$} & \multicolumn{2}{|c|}{$\left(\mathrm{H}_{2} \mathrm{O}\right)_{3} \mathrm{OH}^{-}$} \\
\hline degeneracy & $\begin{array}{l}\text { frequency } \\
\left(\mathrm{cm}^{-1}\right)\end{array}$ & degeneracy & $\begin{array}{c}\text { Erequency } \\
\left(\mathrm{Cm}^{-1}\right)\end{array}$ \\
\hline 1 & 2840 & 1 & 3670 \\
\hline 2 & 2540 & 2 & 1025 \\
\hline 1 & 1140 & 3 & 2790 \\
\hline 2 & 1630 & 3 & 3440 \\
\hline 1 & 580 & 3 & 1560 \\
\hline 2 & 610 & 1 & 750 \\
\hline \multirow[t]{5}{*}{3} & 200 & 2 & 585 \\
\hline & & 1 & 450 \\
\hline & & 2 & 440 \\
\hline & & 3 & 500 \\
\hline & & 12 & 200 \\
\hline
\end{tabular}

amost frequencies are given by Kresge et al. Phonon frequencies are taken to be $200 \mathrm{~cm}^{-1}$. Three librational frequencies of $500 \mathrm{~cm}^{-1}$ are assumed for the hydroxide ion. 
of $\mathrm{v}=2,3,4$, and 5 , respectively, are used. Figure $V-14$ shows a best $f i t$ of the experimental points to Equation (16) for values of $\Delta E_{0}$ (laser) equal to $4734 \mathrm{~cm}^{-1}$, $5900 \mathrm{~cm}^{-1}$, and $7041.5 \mathrm{~cm}^{-1}$. Satisfactory fits (within about 208) were obtained for $s$ ranging between 8 and 16 , and $E_{z}$ ranging between $650 \mathrm{~cm}^{-1}$ (the lowest allowed $E_{z}$ ) and $2000 \mathrm{~cm}^{-1}$.

If the values of $S$ and $E_{z r}$ obtained from the fit have physical significance, then in the limit of a large distinction between strongly- and weakly-coupled modes, 8 to 16 modes are strongly coupled and the average energy of the modes is between $160 \mathrm{~cm}^{-1}$ and a few hundred $\mathrm{cm}^{-1}$. The number of strongly-coupled modes is about one-third to one-half the total number of modes in a three or four molecule linear chain.

Knight et al. 64 used an expression of the form

$$
\phi=A\left[\left(E-E_{a}\right) / E\right]^{S}
$$

to fit the temperature and wavelength dependence of the quantum yield, where $E$ is the photon energy, $E_{a}$ is the barrier, $s$ is the number of coupled modes, and $A$ is $a$ proportionality constant. The temperature dependence in this model comes from the temperature dependence of $\mathrm{E}_{\mathrm{a}}$. Additional temperature dependence expected from the ion pair separation step is not included in the Knight model. There are several important differences between the Knight model, and the model reported here. Knight assumes an RRK reaction rate with an exponential energy relaxation. 
The activation energy is given by $\Delta H$, the reaction endothermicity. They assume recombination in the ion pair is negligible. The high level of excitation and low reverse barrier for geminate recombination suggest that recombination may be important. The model reported here assumes recombination in the ion pair is rapid enough to produce local equilibrium. Also, the relaxation rate is assumed to be constant or weakly dependent on the level of excitation.

In spite of the differences between the model reported here and knight's model, the resulting expressions are similar. The value knight found for of $s$, the number of coupled modes, is 8.4 ; within the $8-16$ range determined here.

The fit of the experimental data to equation (16) can be used to estimate if bend excitation is as efficient as stretch excitation in producing the vibrationally activated reaction. Between 508 and 808 of the absorption at the stretch and bend combination band near $11,800 \mathrm{~cm}^{-1}$ is from underlying $\mathrm{v}=3 \mathrm{O}-\mathrm{H}$ stretch excitation. Equation 16 predicts a quantum yield of $5.7 \times 10^{-7}$ for excitation of pure $v=3$ OH stretch and $2.7 \times 10^{-6}$ for pure excitation of the 3 stretch +1 bend combination. Thus, the predicted quantum yield at $11,800 \mathrm{~cm}^{-1}$ is between $1.64 \times 10^{-6}$ and $1.0 \times 10^{-6}$. This compares to a measured guantum yield of $1.75 \times 10^{-6}$. Within experimental error, excitation of the bend is as efficient as excitation of the stretch. 
One additional model for the photon energy dependence of the quantum yield was tested. If the vibrationally activated laser reaction comes about from a small locally hot region near the initially absorbed photon, then one might expect a Boltzmann quantum yield dependence,

$$
\phi=A \exp \left(-\Delta H n / E_{\lambda}\right),
$$

where $E_{\lambda}$ is the available energy imparted by the photon, $\Delta \mathrm{H}$ is the enthalpy change for the ionization reactions, and $\mathrm{n}$ is the number of modes sharing the energy. The energy available for the ion pair formation reaction is the full photon energy, $E_{p h}$ plus thermal energy, $\mathrm{nRT}$. If the recombination reaction is rapid enough to achieve equilibrium, the energy available for recombination is the photon energy plus thermal energy minus the endothermicity, $E_{\mathrm{ph}}-\Delta \mathrm{H}+\mathrm{nRT}$. Thus, the correct energy to use in equation (27) is between $E_{p h}+n R T$ and $E_{p h}-\Delta H+n R T$. Figure V-15 shows the photon energy dependence predicted by equation (27) for $E_{\lambda}=E_{p h}+n R T$ and $E_{\lambda}=E_{p h}-\Delta H+n R T$ along with the experimental points. The prediction is made by constraining equation (27) to match experimental values at $16,500 \mathrm{~cm}^{-1}$ and 13240 or $9983 \mathrm{~cm}^{-1}$. With the curve constrained to go through the $13240 \mathrm{~cm}^{-1}$ point, the best fit is with $A=6.91 \times 10^{-3}$ and $\mathrm{n}=23.5$ for $\Delta \mathrm{H}=5075 \mathrm{~cm}^{-1}$ $114.5 \mathrm{kcal})$ when $E_{\lambda}=E_{\mathrm{ph}}+\mathrm{nRT}$. The best fit is $A=5.27 \mathrm{x}$ $10^{-4}$ and $n=7.85$ for $\Delta H=5075 \mathrm{~cm}^{-1}$ when $E_{\lambda}=E_{p h}-\Delta H+n R T$. When the curve is constrained to match experimental points at $9983 \mathrm{~cm}^{-1}$, no real solution exists with $E_{\lambda}=E_{p h}+n R T$. 
For $E_{\lambda}=E_{p h}-\Delta H+n R T$, the best fit is with $A=1.38 \times 10^{-3}$ and $n=10.76$. The small value for $n$ in each case supports the hypothesis that a small number of water molecules is involved as was found in the other wavelength dependence models. The curve with $E_{\lambda}=E_{p h}-\Delta H+n R T$ matches the experimental points more closely than $E_{\lambda}=E_{p h}+n R T$. 
Figure $\mathrm{V}-15$

Experimental points at $10 \pm 1^{\circ} \mathrm{C}$ for the quantum yield versus excitation wavelength, (0), along with three fits to $\phi=A \exp \left(-\Delta H n / E_{\lambda}\right)$, where $\Delta H=5075 \mathrm{~cm}^{-1}$ $\{14.5 \mathrm{kcal}\}$. The parameters used are $\mathrm{A}=6.91 \times 10^{-3}$ and $\mathrm{n}=23.5\left(\longrightarrow\right.$ for $\mathrm{E}_{\lambda}=\mathrm{E}_{\mathrm{ph}}+\mathrm{nRT}$. The parameters are $A=5.27 \times 10^{-4}$ and $n=7.85$ for $E_{\lambda}=E_{p h}-\Delta H+n R T$, $(--)$. The curve $(--)$ is $\mathrm{A}=1.38 \times 10^{-3}$ and $\mathrm{n}=10.76$, with $E_{\lambda}=E_{p h}-\Delta H+n R T$. 


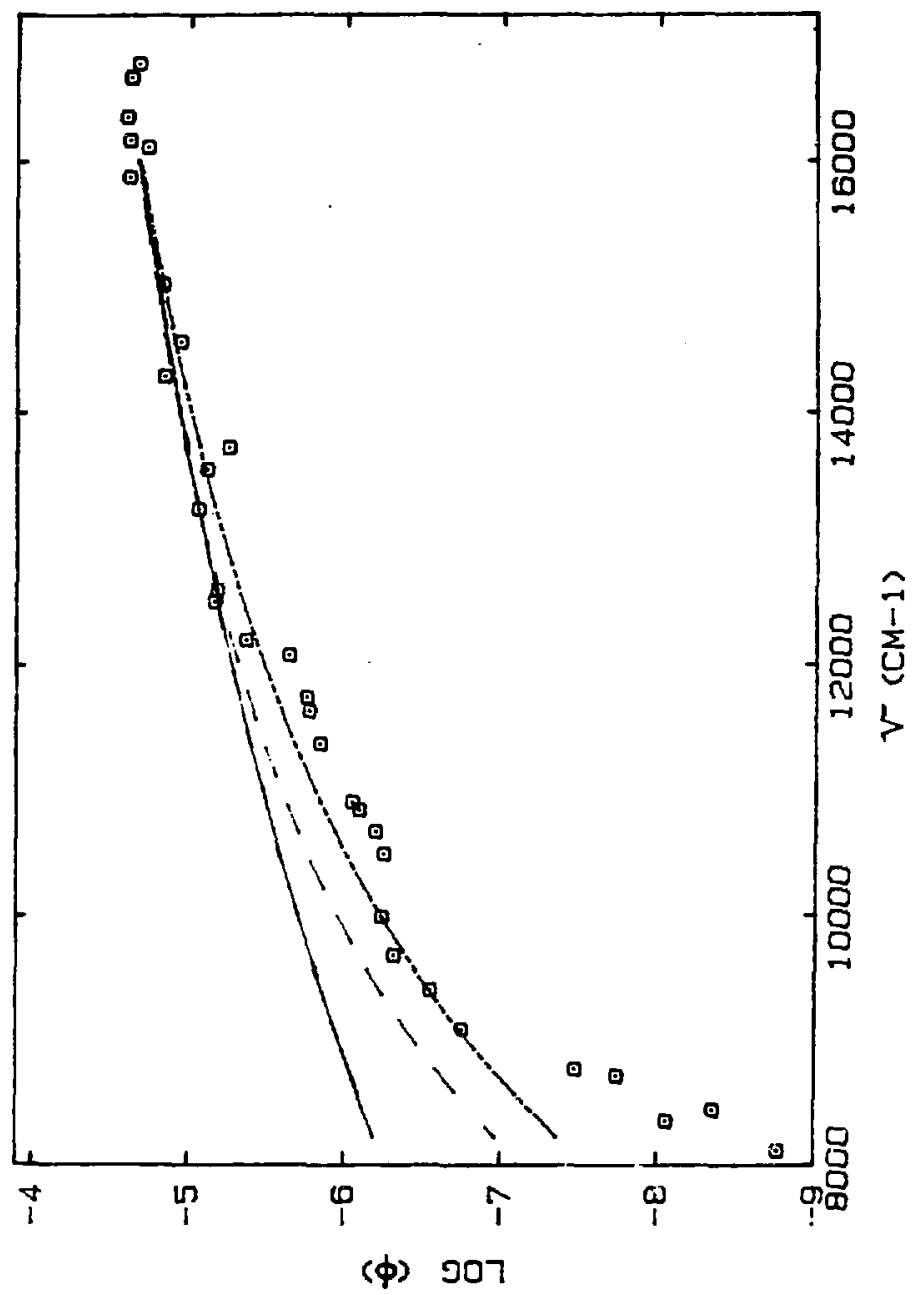


1. M. Eigen and L. De Maeyer, Z. Elektrochem. 59, 986 (1955)

2. M. Eigen and L. De Maeyer, Proc. Roy. Soc. (London), Ser. A 247,505 (1958)

3. J.F. Holzwarth, W. Frisch, A.J. Kresge and D.M. Goodall, to be published

4. G.A. Vidulich, D.F. Evans, and R.L. Kay, J. Phys. Chem. 71, 656 (1967)

5. V.G. Ertl and H. Gerischer, 2. Elektrochem., $\underline{66}$ $560(1962)$

6. G.C. Barker, P. Fowles, D.C. Sammon, and B. Stringer, Trans. Faraday Soc. 66,1498 (1970)

7. A.H. Narten, M.D. Danford, and H.A. Levy, Disc. Faraday Soc. 43,97 (1967)

8. D. Hankins, J.W. Moskowitz, and F.H. Stillinger, J. Chem. Phys. 53, 4544 (1970); Erratum, 59, 995 (1973)

9. J. O'M. Bockris and A.K.N. Reddy, "Modern Electrochemistry" v. I, Plenum, New York (1979) Chapter 5

10. F.H. Stillinger in "Theoretical Chemistry; Advances and Perspectives", v. 3, Academic Press, New York (1978)

11. R. Triolo and A.H. Narten, J. Chem. Phys 63, 3624 (1975)

12. I. Olovsson, J. Chem. Phys. 49, 1063 (1968)

13. J.-0. Lundgren and I. Olovsson, Acta Crystallogr. 23, 966,971 (1967) 
14. J.-O. Lundgren and I. Olovssor, T. Chem. Phys. $\underline{49}$, 1068 (1968)

15. R.A. Bell, G.G. Christoph, F.R. Fronczek, and R.E. Marsh, Science 190, 151 (1975)

16. M.D. Newton and S. Ehrenson, J. Am. Chem. Soc. 93, 4971 (1971)

17. G. Beurskens and G.A. Jeffrey, J. Chem. Phys. 4I, 924 (1964)

18. R.K. McMullan, T.C.W. Mak, and G.A. Jeffrey, J, Chem. Phys. 44, 2338 (1966)

19. R.A. More O-Eerrall in "Proton Transfer Reactions" E.F. Caldin and V. Gold eds., Chapman and Hall, London (1975) pp. 218, 320

20. S.A. Rice and M.G. Sceats, J. Phys. Chem, 85, 1108 (19B1)

21. M.G. Sceats and K. Belsley, Mol. Phys. 40, 1389 (1980)

22. M.F. Vernon, D.J. Krajnovich, H.S. Kwok, J.M. Lisy, Y.R. Shen, and Y.T. Lee, J. Chem. Phys., submitted

23. N. Koroteev, M. Endemann, and R. Byer, Phys. Rev. Lett. 43,398 (1979)

24. J. Arnold and D.J. Millen, J. Chem. Soc., 503 (1965)

25. J.E. Bertie and D.J. Millen, J. Chem. Soc., 497 (1965)

26. C.A. Coulson and G.N. Robertson, Proc. R. Soc. Lond. A 342, 289 (1975)

27. Y. Bouteiller and Y. Guissani, Mol. Phys. 38, 617, (1979) 
28. L.E. Brus and V.E. Bondybey in "Radiationless Transitions", Academic Press, S.H. Lin, ed. (1980)

29. B.S. Rabinovitch and D.W. Setser, Advan. Photochen. 3 , 1 (1964)

30. W.J. Albery in "Proton Transfer Reactions", Chapman and Hall, E.F. Caldin and V. Gold, eds. (1975)

31. M. yon Smoluchowski, Phys. 2. 17, 557, 585 (1916)

32. M. yon Smoluchowski, 2. Physik. Chem. (Leipzig) 92, 129 (1917)

33. I. Onsager, J. Chem. Phys. 2, 599 (1934)

34. K.M. Hong and J. Noolandi, J. Chem. Phys. 68, 5163 (1978)

35. reference 9, chapter 4, equation 4.169 .

36. reference 9

37 P.J. Robinson and K.A. Holbrook, "Unimolecular Reactions" Wiley-Interscience, London, (1972), section 5

38. David . Oxtoby in "Photoselective Chemistry", Adv. Chem. Phys., v. XLVII pt. 2, p. 487

39. R.R. Alfano and S.L. Shapiro, Phys, Rev. Lett. 29, 1655 (1972)

40. A. Fendt, S.F. Fischer, and W. Kaiser, Chem. Phys. 57, 55 (1981)

41. A. Fendt, S.F. Fischer, and W. Kaiser, Chem. Phys. Lett. 82,350 (1981)

42. A. Laubereau, S.F. Fischer, K. Spanner, and W. Kaiser, Chem. Phys. 31, 335 (1978) 
43. S.A. Rice and M.G. Sceats, J. Phys. Chem. 85, 1108 (1981)

44. R. McGraw, W.G. Madden, M.S. Bergren, S.A. Rice, and M.G. Sceats, J. Chem. Phys. 69, 3483 (1978)

45. W.G. Madden, M.S. Bergren, R. McGraw, S.A. Rice, and M.G. Sceats, J. Chem. Phys. 69, 3497 (1978)

46. M.S. Bergren and S.A. Rice, J. Chem. Phys. 77, 583 (1982)

47. E.B. Wilson, J.C. Decius, and P.C. Cross, Molecular Vibrations", McGraw Hill, New York (1955) section 8.5

48. Handbook of Chemistry and Physics, 53rd Edition p. E-11

49. R.A. More O'Ferrall, G.W. Koeppl, and A.J. Kresge, J. Am. Chem. Soc. 93, 1 (1971)

50. R.A. More O'ferrall, G.W. Koeppl, and A.J. Kresge, J. Am. Chem. Soc. 93, 9 (1971)

51. R.A. More O'Ferrall and A.J. Kresge, J.C.S. Perkin II, 1840 (1980)

52. G.E. Walrafen, J. Chem. Phys. 47, 114 (1967)

53. G.E. Walrafen, J. Chem. Phys. 48, 244 (1968)

54. G.E. Walrafen in "Hydrogen-Bonded Solvent Systems", A.K. Covington and P. Jones, eds., Taylor and Francis, London, (1968), p. 9

55. R.E. Weston, Jr., Spectrochim. Acta 18, 1257 (1962)

56. P.A. Giguere, Rev. Chim. Miner. 3, 627 (1966)

57. K. Heinzineger and R.E. Weston, Jr., J. Phys. Chem. 68, 744 (1964) 
58. L.H. Jones, J. Chem. Phys 22, 217 (1954)

59. I. Gennick and K. Harmon, Inorg. Chem. 14, 2214 (1975)

60. E. Drouard, Compt. rend. 247, 68 (1958)

61. J.O. Clifford, J.A.S. Smith, and F.A. Temme, J.C.S. Faraday 11, 1352 (1975)

62. P. Rhine, D. Williams, G.M. Hale, and M.R. Querry, J. Phys. Chem. 78, 1405 (1974)

63. W.R. Busing and D.F. Hornig, ibid, 65, 284 (1961)

64. B. Knight, D.M. Goodall, and R.C. Greenhaow, J. Chem. Soc. Faraday 2 75, 841 (1979) 


\section{CHAPTER VI. CONCLUSIONS}

The conclusions are divided into two parts: (A) major conclusions based on the thermal equilibrium relaxation rate; and (B) major and minor conclusions based on quantum yields for the vibrationally activated reaction.

\section{Thermal Recombination Reaction}

The recombination distance is constant with isotopic composition and temperature with a distance of $5.80 \pm 0.5 \mathrm{~A}$. The activation energy for the recombination reaction is $3.5 \mathrm{kcal} / \mathrm{mole}$.

II. Vibrationally Activated Reaction

First, the model independent conclusions will be given. The major model independent conclusion is that single photon overtone excitation can be used to vibrationally activate a liquid phase reaction. Excited liquid water transfers a proton to form $\mathrm{H}_{3} \mathrm{O}^{+}$and $\mathrm{OH}^{-}$. The quantum yield has the same general wavelength dependence for excitation of $\mathrm{H}_{2} \mathrm{O}$ or $\mathrm{D}_{2} \mathrm{O}$, but is shifted to lower quantum yields in $\mathrm{D}_{2} \mathrm{O}$. The hydrogen mole fraction dependence of the quantum yield and the wavelength dependence for $v=3$ OH stretch or $v=4$ OD stretch excitation in 0.017 mole fraction hydrogen in $D_{2} O$ is 
consistent with a lower quantum yield for HOD excitation in $D_{2} \mathrm{O}$ than for $D_{2} \mathrm{O}$ excitation in $D_{2} \mathrm{O}$. A temperature increase causes a smaller fractional quantum yield increase for large excitation energies than for small excitation energies. The constancy of quantum yield with wavelength across an $\mathrm{OH}$ stretch absorption band results from one of two possibilities depending on the source of broadening in the absorption band. If the broadening comes from differences in liquid structure, then the quantum yield is independent of those structural differences. If the broadening comes from combination excitation of low frequency lattice motions, then excitation of lattice motions does not produce reaction products as efficiently as the equivalent energy in $0-H$ stretch motions.

The major model dependent result is that the minimum in the quantum yield as a function of hydrogen mole fraction results from an increase in relaxation rate or from an unfavorable change in the relaxation pathway for HOD in $D_{2} O$. The two possibilities cannot be distinguished. In addition, the diffusive ion pair separation probably occurs at an effective temperature higher than ambient. An empirical fit of the quantum yield versus wavelength was given. In the limit of large distinction between strongly and weakly coupled modes, the fit shows about one-third to one-half of the modes in a 3-4 molecule 
linear chain are strongly coupled, and the zero point energy involves modes with low average frequency. The physical validity of the fit must be tested by experiments on other systems. Excitation of bend modes is as efficient in producing the vibrationally activated reaction as excitation of stretch. 


\section{APPENDIX A}

This sections demonstrates that the absolute transient voltages are independent of the position and intensity distribution of the illuminated region for a uniform interelectrode electric field. Figure A-1 shows a schematic diagram of tise interelectrode region and the equivalent electrical circuit. Region 2 is the illuminated region.

It is easy to show the relative signal magnitudes are independent of the position of the illuminated region. The following statements hold independent of position.

1. The temperature change in the illuminated region is constant.

2. The number of ions produced by the laser reaction is constant.

3. The change in ionic conductivities and equilibrium $\mathrm{H}^{+}$and $\mathrm{OH}^{-}$concentrations produced by the temperature increase is constant.

Therefore, the changes in solution conductivity and the relative magnitude of the transient voltage peak and baseline step are independent of the position of the illuminated region.

The absolute transient voltage change is also independent of position, spot size, and intensity profile for a uniform interelectrode electric field. In all experiments, the illuminated region is $\phi$, the central interelectrode region where the electric field is most 
Figure A-1

A schematic diagram of the interelectrode region of the illuminated electrode pair, and the equivalent electrical circuit. 

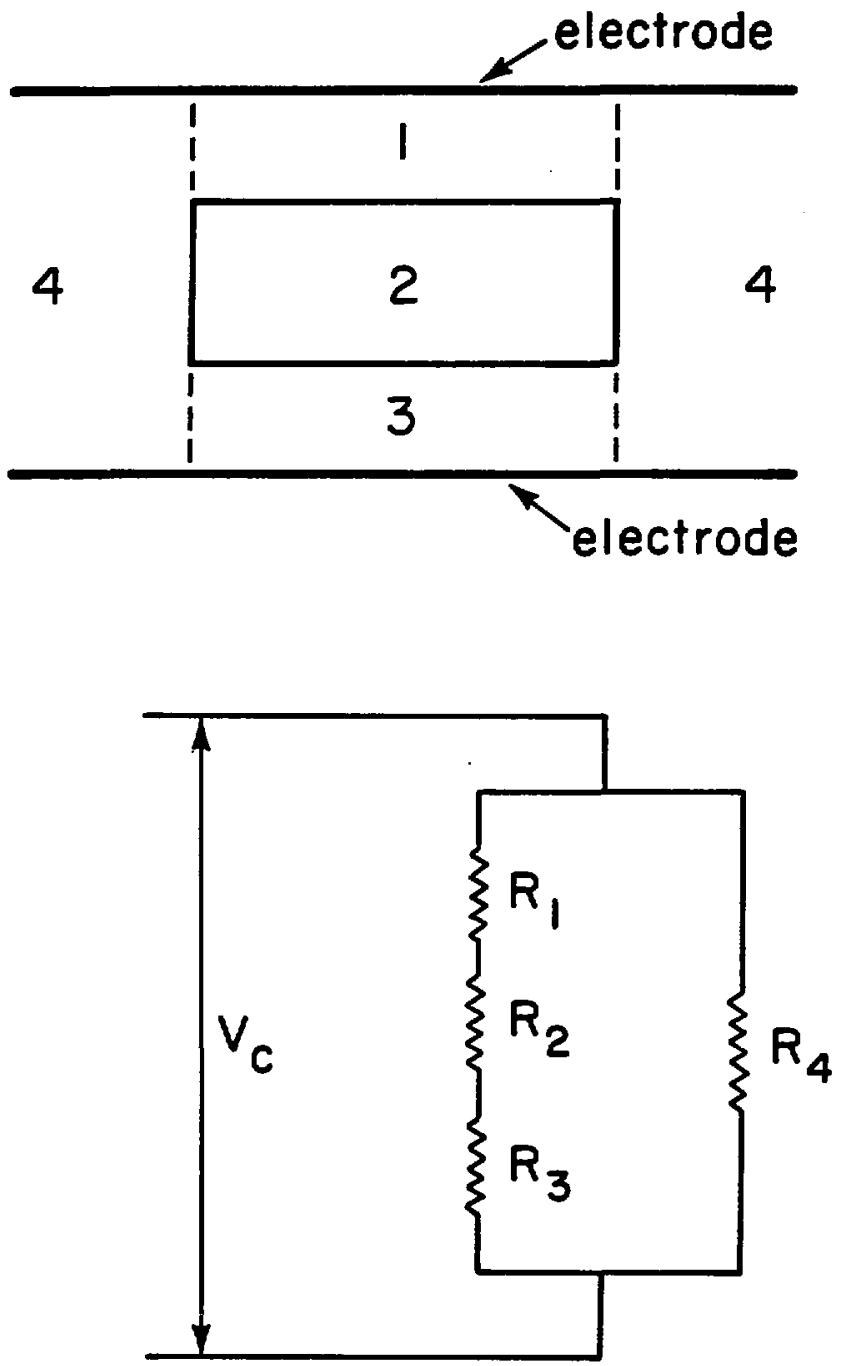

XBL 831-7708 
uniform. Field uniformity means that resistances are dependent only upon geometry. The resistance, $\mathrm{R}=\ell / \mathrm{AL}$. $L$ is the solution conductivity in the region of interest, $A$ is the area of the face parallel to the electrode and $\&$ is the thickness.

Referring to the circuit equivalent of the electrode paic shown in Figure $A-1$, the total resistance of the electrode is $R C 2=R_{4}\left(R_{1}+R_{2}+R_{3}\right) / R_{1}+R_{2}+R_{3}+R_{4}$. The quantity of interest is the current change given a change in resistance of the illuminated region. Equation (32) of the analysis section gives

$$
i_{c}=v_{c} / R C 2
$$

where $i_{c}$ is the current through the cell of resistance RC2 given an applied voltage of $V_{C}$. The current change is

$$
\Delta i_{c}=\frac{d v_{c} / R C 2}{d R_{2}} \Delta R_{2}
$$

Replacing RC2 and taking the derivative, (2) becomes

$$
\Delta i_{c}=\frac{-V_{c}}{\left(R_{1}+R_{2}+R_{3}\right)^{2}} \Delta R_{2}
$$

Replacing the resistances with $R=\ell / A L$, and using $1-[1 /(1+x)] \equiv x$ for small $x$.

$$
\Delta i_{c}=\frac{-V_{C} \ell_{2} A}{\left(e_{1}+2_{2}+2_{3}\right)^{2}} \Delta L
$$

Equation (A) shows the absolute signal magnitude is constant with constant interelectrode spacing, 
$\left(\ell_{1}+\ell_{2}+\ell_{3}\right)$, and irradiated spot thickness, $\ell_{2}$. The signal is independent of position, i.e., the relative value of $\ell_{1}$ and $\ell_{3}$,

If the temperature increase is linear in the absorbed energy density and if also, the ionic conductivity and $k_{w}$ is linear in temperature, then the absolute signal magnitude is independent of spot size and beam profile because $\Delta L$ is then inversely proportional to the irradiated volume, $\ell_{2} A$, or $\Delta L \propto \Delta E / \ell_{2} A$ where $\Delta E$ is the total absorbed energy. Half the spot size implies twice the temperature increase, twice the transient ion concentration, and, therefore, twice the conductivity change, $\Delta L$ in the illuminated region. Equation (4) becomes

$$
\Delta i \propto \frac{-v_{C}}{\left(l_{1}+l_{2}+l_{3}\right)^{2}} \quad \Delta E
$$

Equation (5) is independent of spot size and therefore intensity profile. Since the absorbed energy density and resulting temperature increase is small, a linear tempertature, linear ionic conductivity, and linear $\mathbf{k}_{\mathbf{w}}$ with absorbed energy density are excellent approximations. 


\section{Appendix B - ABQMHD.WES}

Program to calculate the quantum yield for dissociative ionization of water in an arbitrary isotopic mixture.

This program calculates the quantum yield from both the measured transient voltage step and from the absorbance and transmitted laser energy. Comments for the program and subroutine functions, a definition of variables used in the program, and the program text are given.

A. Program comments

10 Open a file for hard copy output

15 Open an output file for results

50-450 Input data

460-480 Calculate quantum yields, and write the intermediate results to the hard copy output file

490

Form hardcopy and output files for Einal quantum yield results based on the transient voltage step and for quantum yield results based on the absorbance and transmitted energy .

B. Subroutine function 
1230

$\& 1810$ Read raw data for analysis

$\$ 440$ Input routine for the thermocouple voltage

$1 ; 40$ Input routine for the wavelength

1740 Input routine for the monitor voltage

1915 Open an output file

- 100 Read data from a previously analyzed file for re-analysis

C. Definition of variables

$\begin{array}{ll}\text { Al } & \text { absorbance of water } \\ \text { factor for calculating the baseline step from } & \text { absorption spectra } \\ \text { Aj } & \text { conductivity step from absorbance and } \\ & \text { transmitted laser energy } \\ \text { 48 } & \text { conductivity step from transient voltage step } \\ \text { A9 } & \text { conductivity jump } \\ \text { F } 7 & \text { total conductivity jump-tinermal jump, } \\ & \text { induced reaction (based on t.te absorbance } \\ \text { D2 } & \text { and transmitted laser energy) } \\ & \text { total conductivity jump-thermal jump, or } \\ & \text { portion of jump caused by the laser induced } \\ & \text { reaction (based on the transient voltage step) } \\ & \end{array}$




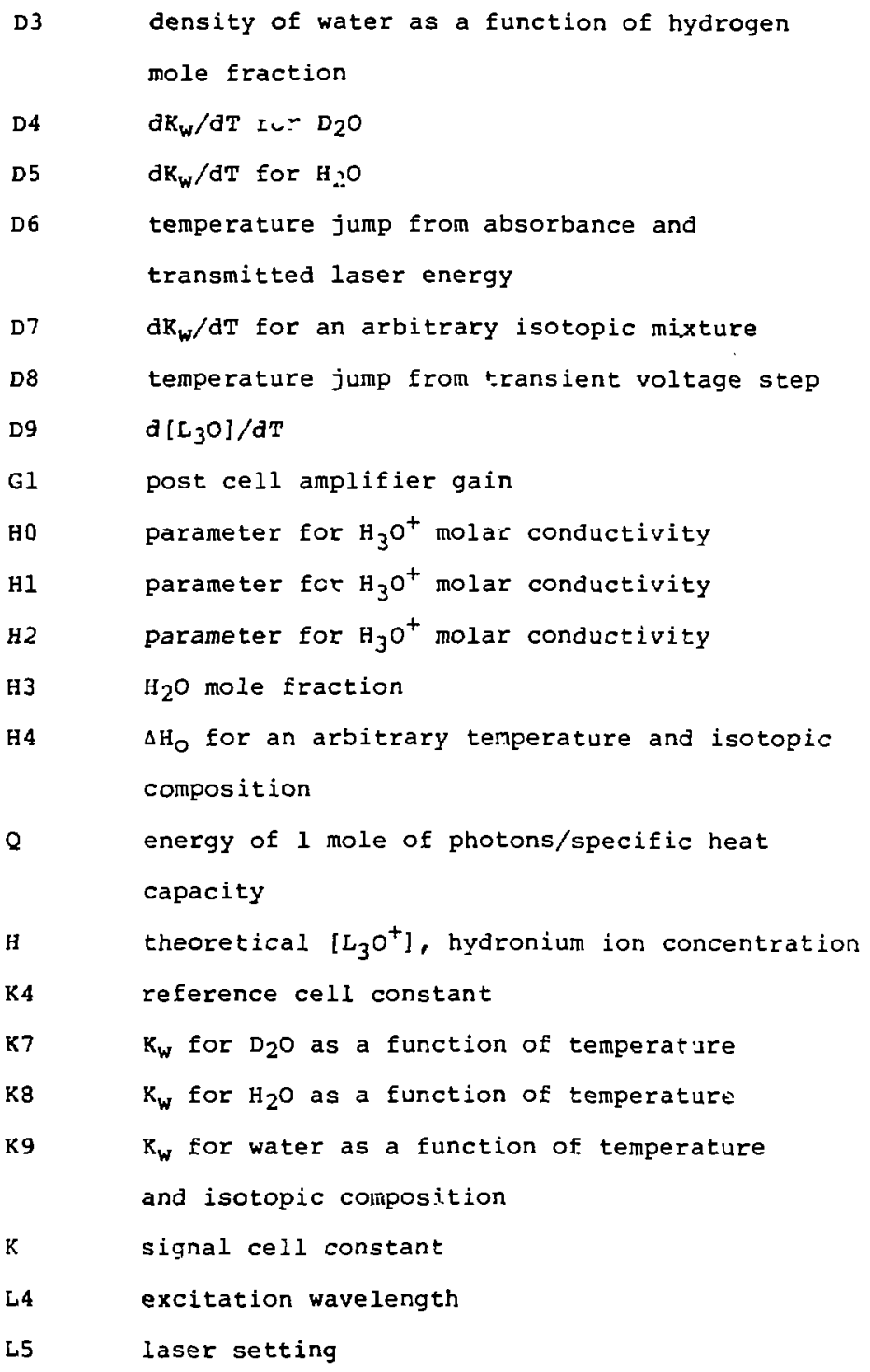


L

M4

M5

M6

M7

M8

M

No

N1

N2

N3

N9

P1

P4

Q7

Q9

$Q$ experimental conductivity of solution

$\mathrm{d \lambda}\left(\mathrm{I}_{3} \mathrm{O}^{+}+\mathrm{OL}^{-}\right) / \mathrm{dT}$ in an arbitrary isotopic

mixture

dג $\left(\mathrm{Na}^{+}+\mathrm{Cl}-\right) / \mathrm{dT}$ in an arbitrary isotopic

mixture

mole fraction hydrogen

ic uic conductivity of $\mathrm{H}_{3} \mathrm{O}^{+}+\mathrm{OH}^{-}$at $25^{\circ} \mathrm{C}$ as

a function of hydrogen mole fraction ionic conductivity of $\mathrm{H}_{3} \mathrm{O}^{+}+\mathrm{OH}^{-}$as a function

of temperature and hydrogen mole fraction

relaxation rate of the thermal equilibrium

parameter for $\mathrm{NaCl}$ molar conductivity

parameter for $\mathrm{NaCl}$ molar conductivity

parameter for $\mathrm{NaCl}$ molar conductivity

$\mathrm{D}_{2} \mathrm{O}$ viscosity

experimental [ $\left.\mathrm{Na}^{+}\right]$and [Cl-] from excess

conductivity above the theoretical $\left(\mathrm{L}_{3} \mathrm{O}^{+}+\right.$

$\mathrm{OL}^{-}$) conductivity

transmitted laser power

mixture purity

quantum yield based on the absorbance and

transmitted laser energy

quantum yield based on the transient voltage

step

ene:gy of 1 mole of photons/specific heat

capacity 


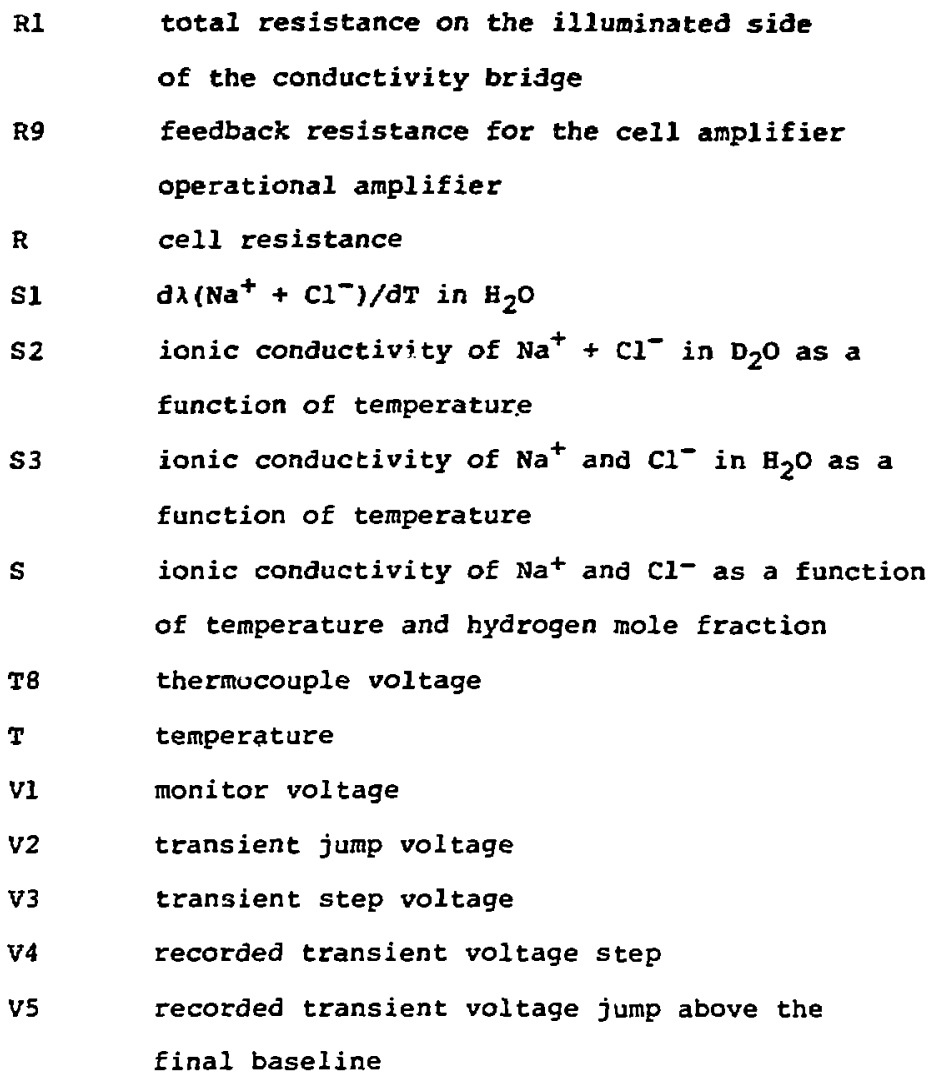

Real voltages are one-half the recorded voltages because the recorded waveform is the waveform at positive polarity minus the waveform at negative polarity. Also, the real transient jump includes the baseline step. transient step voltage calculated from absorbance and transmitted laser energy 
v

พ0

w1

w2

$\mathbf{x I}$

Y 0

YI

Y2 applied voltage

parameter for In $K_{w}\left(\mathrm{H}_{2} \mathrm{O}\right)$ vs. temperature

parameter for In $\mathrm{k}_{\mathrm{w}}\left(\mathrm{H}_{2} \mathrm{O}\right)$ vs. temperature

parameter for $\ln \mathrm{K}_{\mathrm{y}}\left(\mathrm{H}_{2} \mathrm{O}\right)$ vs. temperature

dג $\left(\mathrm{H}_{3} \mathrm{O}^{+}\right) / \mathrm{dT}$ in $\mathrm{H}_{2} \mathrm{O}$

parameter for of $^{-}$molar conductivity

parameter for $\mathrm{OH}^{-}$molar conductivity

hydroxide ionic conductivity in $\mathrm{H}_{2} \mathrm{O}$

D. Program listing (see pages 232-236) 


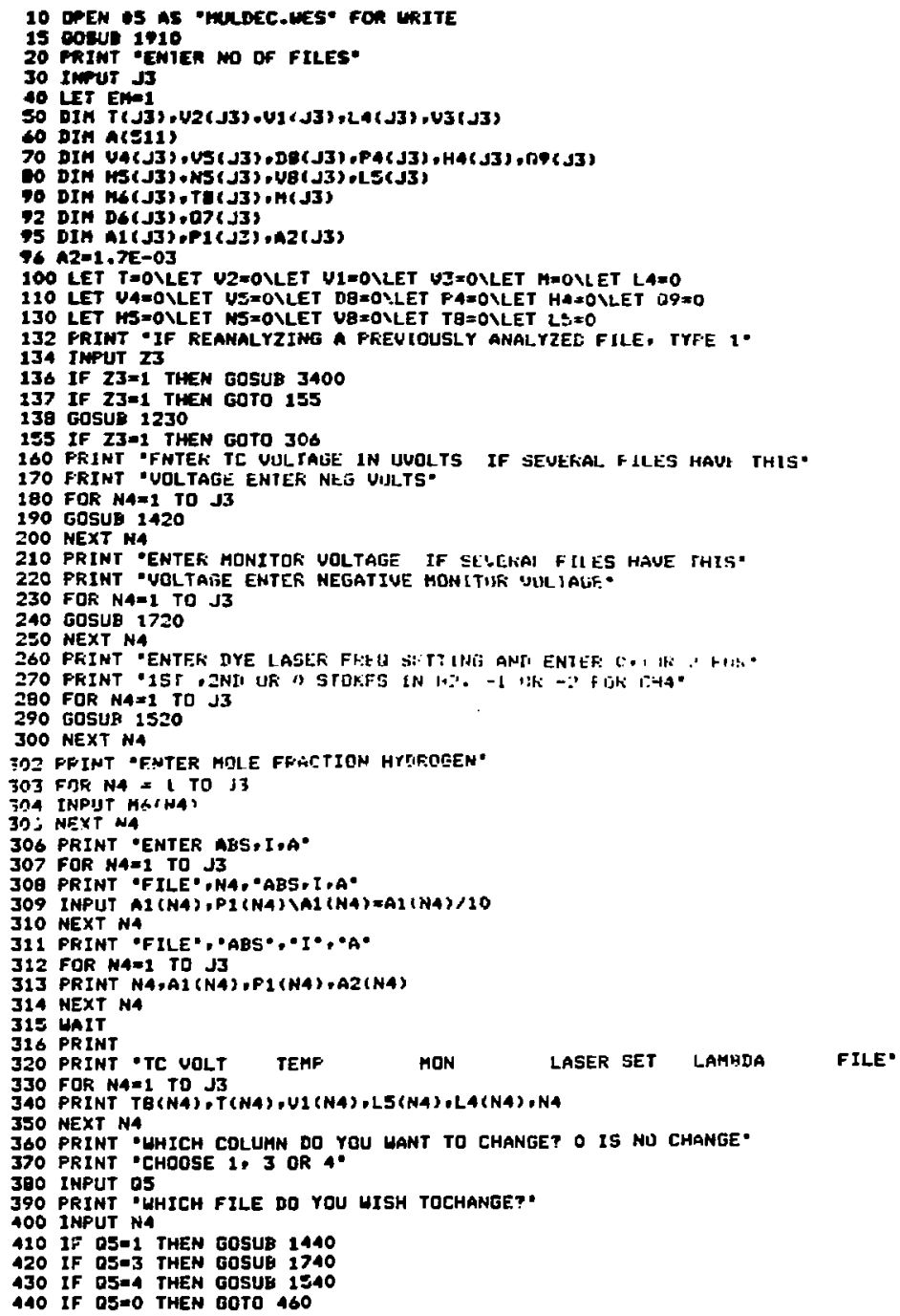




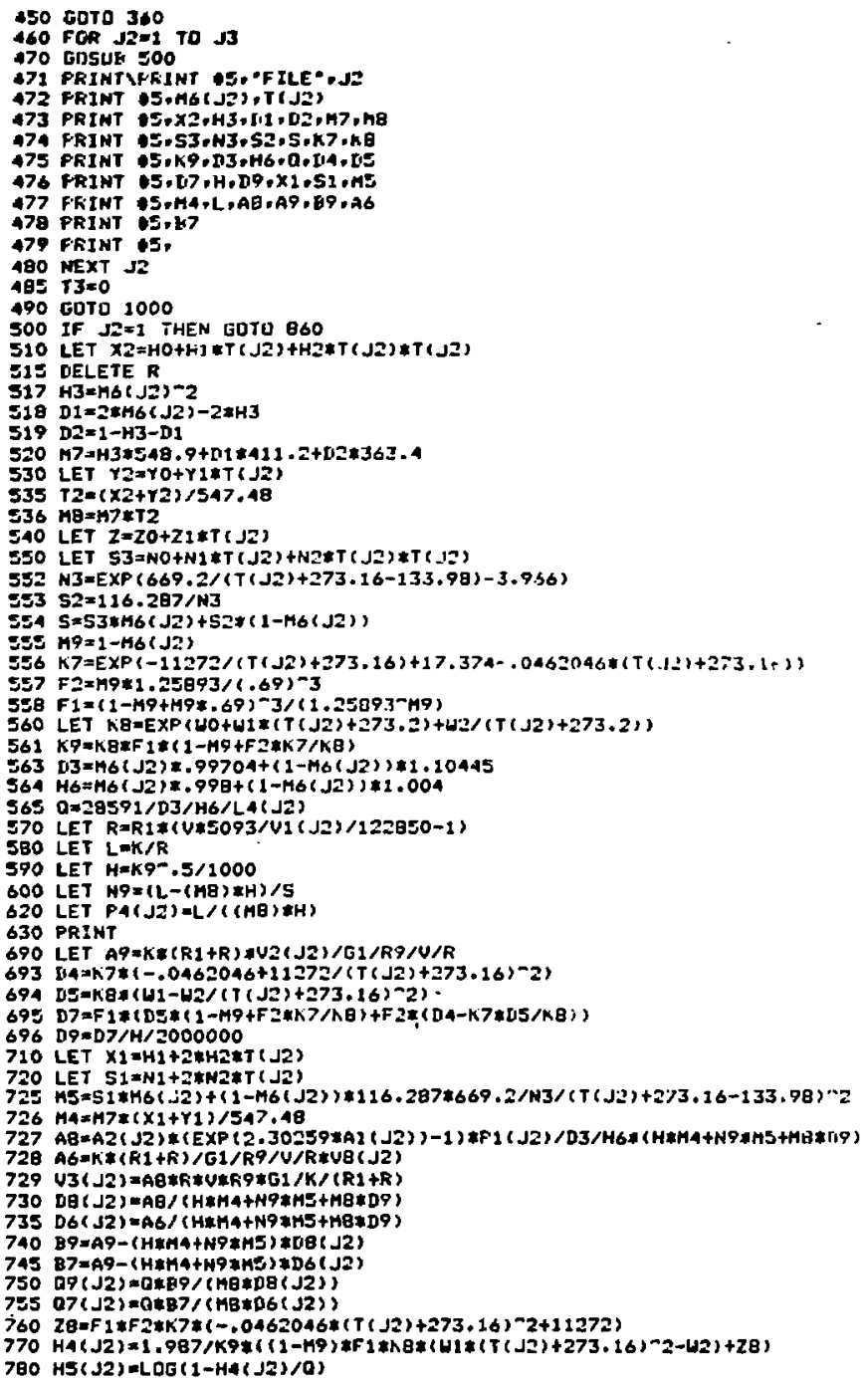




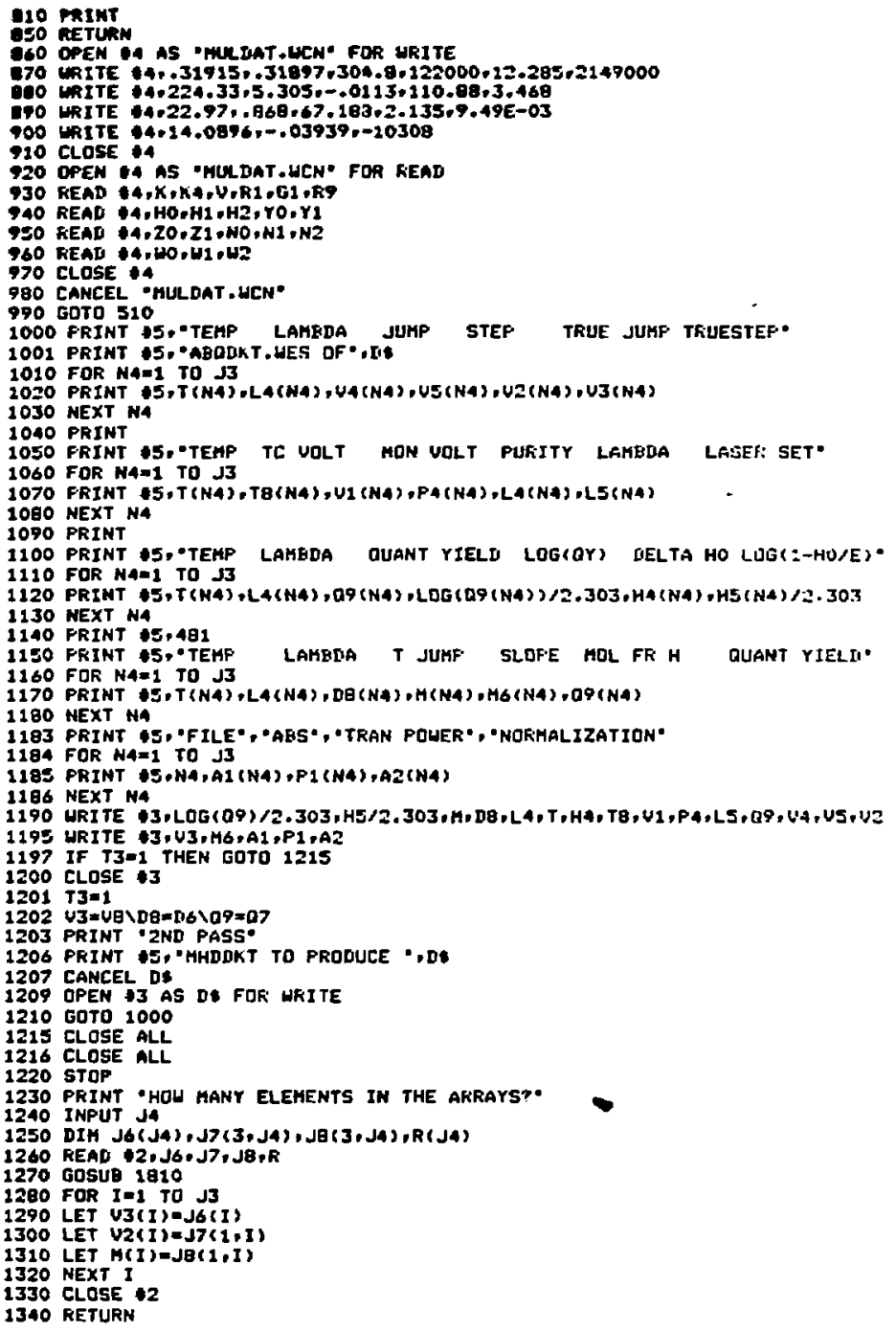




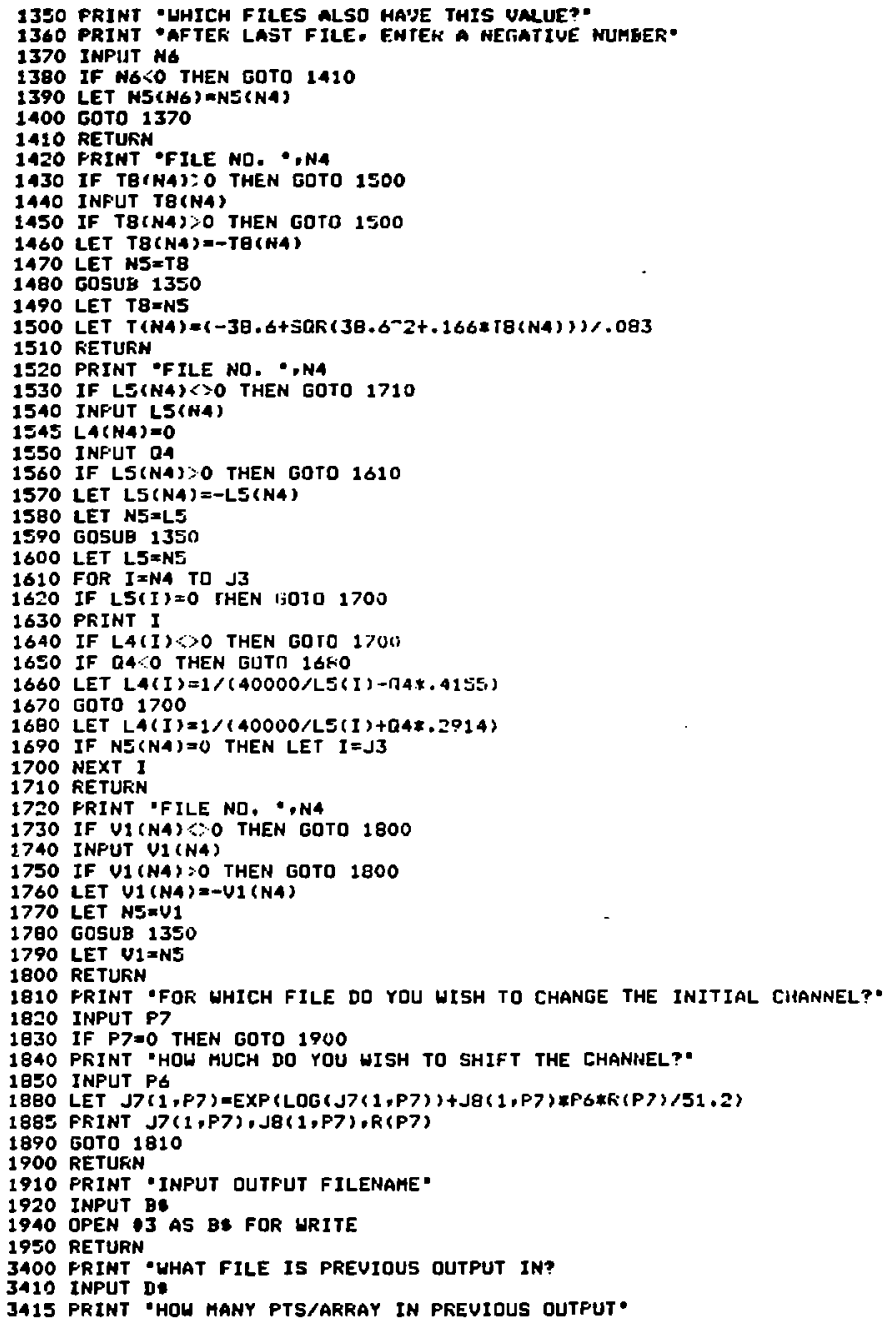


3414 DELETE LA,T.TO,UI,LS

3417 INPUT 27

3419 DIA L4(Z7),T(Z7), TB(Z7),V1(Z7),LE(27)

3420 OPEN 1 AS IT FOR READ

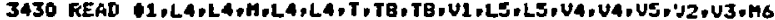

3435 UDEUS/2

3440 CLOSE 1

3450 RETURN 


\section{APPENDIX C - RECDIS.WES}

Program to calculate the recombination distance of $\mathrm{H}_{3} \mathrm{O}^{+}+\mathrm{OH}^{-}$from the equilibrium relaxation rate.

A. Program comments

10 Open an output file for results

20-140 Input raw data

305 Open a file for hard copy output

460-480 Calculate the recombination distance

490 Form hard copy and output files for recombination distance results

B. Subroutine comments

500 calculate the recombination distance
$860-\quad$ Initialize variables
1910 Open an output file
3400 Input raw data from a file previously
created. The hydrogen mole fraction,
relaxation rate, and temperature are
needed for this program.


c. Definition of variables

\begin{tabular}{|c|c|}
\hline D1 & HDO mole fraction \\
\hline D2 & $\mathrm{D}_{2} \mathrm{O}$ mole fraction \\
\hline El & dielectric constant, $E$, for isotopic mixture \\
\hline E2 & dielectric constant for $\mathrm{D}_{2} \mathrm{O}$ \\
\hline E3 & dielectric constant for $\mathrm{a}_{2} \mathrm{O}$ \\
\hline H3 & $\mathrm{H}_{2} \mathrm{O}$ mole fraction \\
\hline $\mathrm{H}$ & theoretical concentration of $\mathrm{L}_{3} \mathrm{O}^{+},\left[\mathrm{L}_{3} \mathrm{O}^{+}\right]$ \\
\hline $\mathrm{K} \mathbf{l}$ & recombination rate \\
\hline KT & $\mathrm{K}_{\mathrm{w}}$ for $\mathrm{D}_{2} \mathrm{O}$ as a function of temperature \\
\hline $\mathrm{K} 8$ & $\mathrm{~K}_{\mathrm{w}}$ for $\mathrm{H}_{2} \mathrm{O}$ as a function of temperature \\
\hline K9 & $\begin{array}{l}\mathrm{K}_{w} \text { for water as a function of temperature } \\
\text { and isotopic composition }\end{array}$ \\
\hline M6 & hydrogen mole fraction \\
\hline M7 & $\begin{array}{l}\text { ionic conductivity of } \mathrm{H}_{3} \mathrm{O}^{+}+\mathrm{OH}^{-} \text {at } 25^{\circ} \mathrm{C} \text { as } \\
\text { a function of hydrogen mole fraction }\end{array}$ \\
\hline M8 & $\begin{array}{l}\text { ionic conductivity of } \mathrm{H}_{3} \mathrm{O}^{+}+\mathrm{OH}^{-} \text {as a function } \\
\text { of temperature and hydrogen mole fraction }\end{array}$ \\
\hline M9 & deuterium mole fraction \\
\hline $\mathbf{s} 2$ & delta times sigma, $\Delta \sigma$ (see discussion) \\
\hline s3 & delta, $\Delta=z_{+} z_{-} e^{2 / 4 \pi \varepsilon_{0} E \sigma k T}$ \\
\hline S4 & recombination distance $=0$, sigma \\
\hline $\mathrm{T} 2$ & $\begin{array}{l}\text { temperature dependence of the ionic } \\
\text { conductivity of } \mathrm{H}_{3} \mathrm{O}^{+}+\mathrm{OH}^{-} \text {in } \mathrm{H}_{2} \mathrm{O}\end{array}$ \\
\hline $\mathrm{ul}$ & diffusion coefficient of $\mathrm{H}^{+}+\mathrm{OH}^{-}$ \\
\hline
\end{tabular}


hydronium ionic conductivity in $\mathrm{H}_{2} \mathrm{O}$

Y2

hydroxide ionic conductivity in $\mathrm{H}_{2} \mathrm{O}$

D. Program listing (see pages 240-241) 
10 GOSUB 1910

20 PRINT "ENTER NO OF FILES"

30 INPUT $\mathrm{J} 3$

40 DIM S4(J3), S3(J3),S2 (J3) +E1 (J3), HB(J3),T(J3),H(J3)

50 DIM U1(J3) +H(J3),MSRJ3).K1(J3.

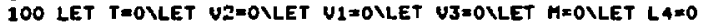

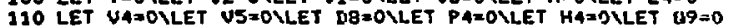

130 LET H5 $=0$ VLET HS $=0$ VLET VB=OLLET TB=0 LLET LS=0

132 FRINT 'IF REANALYZING A PREUIOUSLY ANALYZEN FILE, 1 YI'E. 1*

134 INFUT 73

136 IF $23=1$ THEN GOSUE 3400

137 IF $23=1$ THEN GOTO 140

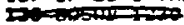

140 FRINT

305 OFEN $\$ 5$ AS MULDEC. HES - FOF HRITE

310 PFIINT

460 FOR $\mathrm{J2}=1$ TO $\mathrm{J3}$

470 GOSUB 500

480 NEXT 12

490 GOTO 1000

500 IF J2=1 THEN GOTO 860

FIO LET XZ=HO+H1*T(J2)+HZ*T(JZ)+T(J2)

E15 DELETE $R$

517 H3=MG $(\mathrm{J} 2)-2$

518 D1 $=2 \pm M G(J 2)-2+43$

$519 \mathrm{DZ}=1-\mathrm{H} 3-\mathrm{N1}$

520 $17=+3 * 548,9+[11 * 411,2+[12 * 363,4$

530 LET $Y 2=Y O+Y 1 * T(J 2)$

$535 T 2=(\times 2+Y 2) / 547.48$

$536 M 8(J 2 ;=M 7 \% T 2$

$555 \mathrm{M9}=\mathrm{I}-\mathrm{Ho}(\mathrm{J2})$

556 K7aEXF $(-11272 /(7(J 2)+273.16)+17.374-.04+2046 * T(J 2)+273.15)$;

$557 \quad F 2=49 \neq 1.25893 /(.69)-3$

558 $F 1=(1-199+M 9 * .69)-3 /(1.2599 .17 H 4)$

560 LET KG=EXF $(w 0+w 1 *(T(J 2)+273.2)+w 2 /(\mathrm{T}(J 2)+173.2)$

561 K9:K8\#F1 (1-M9+F2\#K>/KB)

590 LET H(J2) $=K^{-9} .5 / 1000$

$600 \mathrm{K1}(\mathrm{J} 2)=-1000000 * \mathrm{M}(\mathrm{J} 2) / 2 / \mathrm{H}(\mathrm{J} 2)$

$610 \mathrm{U1}(\mathrm{J} 2)=\mathrm{Mg}(\mathrm{J} 2) \neq 0.930397 \mathrm{E}-10 *(\mathrm{~T}(\mathrm{~J} 2)+273.16)$

620 E2=EXF $\{4.47335-4.6351 E-03 * T(\mathrm{~J} 2)\}$

630 EJ $=E X F(4.47632-4.58445 E-C 3 * T(J 2))$

640 E1 $(J 2)=M 9 * E 2+(1-M 9) \times E 3$

$65052(\sqrt{2})=-1.6711 \mathrm{E}-03 /(\mathrm{T}(\mathrm{J2})+273.16): \mathrm{E} 1(\mathrm{~J} 2)$

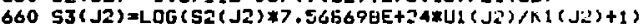

$67054(\sqrt{2})=52(\mathrm{~J} 2) / 53(\mathrm{~J} 2)$

GSO RETURN

860 OPEN 4 AS 'MULDAT. UCN" FOF WRITE

B70 WRITE \$4,.31915,.31897.304,8+122000+12,295.2147000

BBO WRITE $14,224.33,5,305,-.0113,110,69,3,469$

B90 URITE 44,22,97,.868,67,168,2,135,9,49E-03

900 URITE \$ , 14,0996,-.03939,-10306

910 CLOSE 14

920 OPEN 44 AS 'MULDAT. UCN' FOR READ

930 READ $1,, K, K 4, U, R 1, G 1, R 9$

940 READ $4, \mathrm{HO}, \mathrm{H} 1, \mathrm{H}_{2}, \mathrm{YO}, \mathrm{Y} 1$

950 READ $4,20, Z 1, N O, N 1, N 2$

960 READ $44,40,42$, 12

970 CLOSE $\$ 4$

980 CANCEL "HULDAT.WCN"

990 GDTD 510

1000 PRINT "5,"DIFF","MOLE", "RECOMR","RELAX", "K RECOMR" "TEMF"

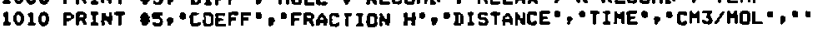

1020 PRIHT IS

1025 FQR N4-1 TO 33

1030 PRINT OS,UI (NA),HG(NA), S4(N4),-1/H(N4),K1(N4),T(N4)

1040 NEXT N4 


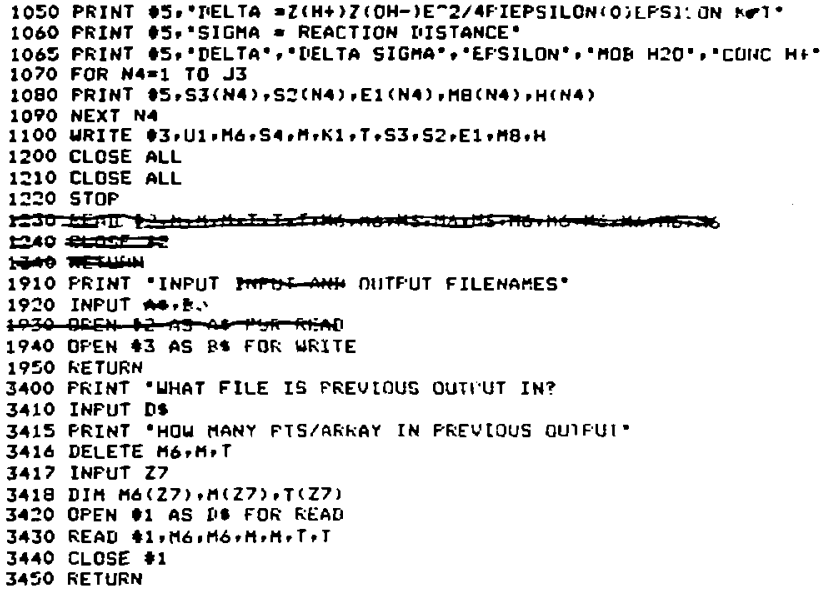




\section{APPENDIX D - Comments on non-statistical effects in overtone photochemistry}

The introduction discusses several attempts to find non-statistical effects in gas phase overtone chemistry. However, a complete unified attempt to demonstrate non-statistical effects following overtone excitation has not been made. For the purposes of the discussion it is important to have a description of the process of overtone absorption (see McDonald).! Assume $\phi_{S}$ is a zero order anharmonic $\mathrm{XH}$ oscillator, local mode state and $\phi_{\ell}$ are all the orthogonal molecular basis functions. In this basis set, \$s has nearly all the overtone oscillator strength. These basis functions are not molecular eigenstates, but the molecular eigenstates, $\phi_{n}$, can be expressed in terms of these basis functions. Or alterna'ively, $\phi_{S}$ can be expressed in terms of the molecular eigenstates. The oscillator strength of an eigenstate is approximately proportional to the square of the $\phi_{S}$ component of that eigenstate. The width of the eigenstate is given by the rate of collisions, radiation, dissociation and any other processes that remove population from the $\phi_{S}$ level.

- It is possible that adjoining eigenstates have differing seaction rates. Classical trajectory studies of model potentials show that it is possible to have both table, repetitive and random, stochastic trajectories in the same energy region. 2,3 If the same behavior exists 
for eigrnstates of real nolecules above the dissociation energy barrier, such qualitatively different trajectories may have gualitatively different dissociation rates and spectroscopic properties. Recent work by Hellez 4 on Raman spectra gives a relationship between classical trajectories and spectroscopic properties. If the molecular state density is low enough, The precision of C.W. dye laser overtone excitation allows excitation of single eigenstates. So far all overtone chemistry experiments use broadband C.W. excitation. Thus, several eigenstates are probably excited simultaneously. As the laser is tuned, only the "average" behavior of the excited eigenstates in a spectral regirn is observed. If only a few eigenstates are not stochastic, then only narrow band excitation will detect non-stochastic behavior.

A second possible way to detect non-statistical behavior is a way first suggested by Moore and Smith.5 They suggested that coherent picosecond excitation of vibrational overtones of reactants in a condensed phase might show non-statistical effects. This suggestion can be given a slightly more quantitative foundation in McDonald's "molecular eigenstates" framework. Assume the reaction rate of the excited state is proportional to the probability that it looks like a local $\mathrm{X}-\mathrm{H}$ stretch. One might imagine this to be true if the projection of the excited $\mathrm{X}-\mathrm{H}$ stretch on the reaction coordinate is large. McDonald shows us that if more than one eigenstate is 
coherently excited, an interference effect is present. The shorter the excitation pulse, the larger the bandwidth of coherently excited eigenstates. The initial constructive interference produces a larger initial $x-H$ stretch probability and therefore a larger initial reaction rate. Thus, the rate at high pressure, where the initial reaction will dominate the total yield, will be higher than the low pressure rate where dissociation of the relaxed species dominates the yield. This could be the quantum mechanical explanation of the Chandle ${ }^{6}$ overtone experiments, 7 and an analogous description almost certainly is the explanation fo the Rabinovitch ${ }^{8}$ cyclopropane experiments. 
1. J.D. McDonald, Ann. Rev. Phys. Chem. 30, 29 (1979)

2. S.A. Rice, Adv. Chem. Phys. 47, part 1, 117 (1981)

3. P. Erumer, Adv. Chem. Phys. 47, part 1, 201 (1981)

4. D.J. Tannor and E.J. Heller, J. Chem. Phys. 77, 202 (1982)

5. C.B. Moore and I.W.M. Smith, Faraday Disc. Chem. Soc. 67, 146 (1979)

6. D.W. Chandler, W.E. Farneth, and R.N. Zare, J. Chem. Phys. 77, 4447 (1982)

7. This assumes a C.W. dye laser can coherently excite more than one eigenstate. If the dye laser cannot, then the experimental result is surprising indeed. If eigenstates are incoherently, individually excited, there can be not time dependence in the dissociation rate.

8. J.D. Rynbrandt and B.S. Rabinovitch, J. Them. Phys. $\underline{54}, 2275(19)$ 


\section{APPENDIX E - FURTHER WORK}

This section suggests further work on aqueous systens, outlines how data from these systems can be analyzed, and suggests some completely new but related systems.

Aqueous Systems

Measuring quantum yields for liquid mixtures other than $\mathrm{H}_{2} \mathrm{O} / \mathrm{D}_{2} \mathrm{O}$ mixtures could provide useful information. Potentially helpful references include 1 through 5. A mixture of $\mathrm{H}_{2} \mathrm{O}$ and $\mathrm{H}_{2} \mathrm{O}_{2}$ can produce a "broken" link in a linear chain of $\mathrm{H}_{2} \mathrm{O}$ molecules without too great a disruption of physical or of vibrational structure. A mixture of $\mathrm{H}_{2} \mathrm{O}$ and alcohol, $\mathrm{ROH}$, will alter mixture conductivities and perhaps provide an efficient relaxation path through the $\mathbf{R}$ group. Also, several solutes which do not hydrogen bond to the water can be tried, such as acetonitrile, acetone, or $\mathrm{CBr}_{2} \mathrm{~F}_{2}$. The $\mathrm{CBr}_{2} \mathrm{~F}_{2}$ has no high frequency modes that can couple to the or stretch of the excited water.

Analysis of data from these systems cannot be done by the same method used for pure $\mathrm{H}_{2} \mathrm{O}$. Insufficient information is available in the literature. The number of photons absorbed by $\mathrm{H}_{2} \mathrm{O}$ can be determined from the spectroscopy of the water/solute mixture and the transmitted laser intensity. If product ion conductivities are known, the number of reactive events can be determined 
from the portion the transient conductivity peak resulting from laser induced reaction. Product ion conductivities for a particular mixture can be determined with adeguate accuracy from a conductivity bridge and solutions of $\mathrm{KOH}, \mathrm{HCl}$, and $\mathrm{KCl}$. The portion of the conductivity peak caused by the laser induced reaction can be determined by subtracting a multiple of the thermal curve obtained by tuning the laser to a wavelength longer than about $1.3 \mu$ where there is no laser induced reaction. The multiple is chosen so that the thermal baseline step is eliminated. Absolute yields can be determined by calibrating with pure $\mathrm{H}_{2} \mathrm{O}$.

Non-aqueous systems

Two non-aqueous systems would be interesting to try. The first is neat liquid ammonia. Liquid ammonia is a weakly dissociating, hydrogen bonded liquid similar to water. It would be interesting to know if liquid water is the only system which shows a laser induced reaction. (Preliminary experiments on neat MeOH showed no discernable conductivity peak.)

The other non-aqueous system is an entirely different class of condensed phase system that may avoid some of the disadvantages of the $\mathrm{H}_{2} \mathrm{O}$ system. Proton transfer in acid-base pairs isolated in a Shpolskii matrix of n-paraffins ${ }^{7}$ could be activated by overtone absorption. 
Electronic spectra of dye molecules in an n-paraffin matrix at $20^{\circ} \mathrm{K}$ of ten show a sharp $\mathrm{I}$ ine spectra with about $1 \mathrm{~cm}^{-1}$ width instead of the usual braod band structure present in a liquid. Spectral analysis shows that a Shpolskii matrix possess a few inequivalent sites which do not interact strongly with the guest molecule. Since the fluorescence excitation spectra are sharp, both the ground and excited state must be sharp, 8,9 and it is reasonable to expect the overtone spectra to be sharp as well. Ideally, if a Shpolskii type of matrix can be made of per-halogenated oils, then any potential interference from host overtone absorption can be eliminated.

If acidic dye molecules are introduced with an excess of base, or if basic dye molecules are introduced with an excess of acid, it should be possible to form acid-base pairs in the low dielectric constant paraffins.10 Thus, there will be two potential wells for the proton; one on the acid and one on the base. As the temperature is lowered, the proton should lie in the lowest well. Alternatively, a molecule such as methyl salicylate, with an intramolecule proton transfer, can be used. Many strongly fluorescing dye molecules show wavelength differences in an acidic versus a basic form, $9,10-13$ These should be unambiguously resolved in the Shpolskii matrix and can thus serve as a probe of the relative concentration of the acidic and basic form of the dye. 
The experiment is carried out by using a C.w. dye laser to probe the concentration of the highest enthalpy form of the dye molecule. Upon initial formation of the matrix, the low temperature ensures that most of the dye molecules are in the low enthalpy form. Very few high enthalpy molecules will be thermally excited and fluorescence will be weak or non-existant. A pulsed dye laser is used to excite overtones of the matrix isolated dye molecule. A fraction of the excited acid-base fairs can transfer a proton to produce the high enthalpy form if the photon energy of the pulsed dye laser is above the energy barrier. These molecules will absorb C.w. dye laser photons and fluoresce. The rise and fall of the fluorescence signal serves as a monitor of the time dependent concentration of the high enthalpy dye molecules.

The same sensitivity advantages obtained in the liquid water reaction by making a transient rather than a C.W. measurement are obtained, but fewer disadvantages are present.

The spectroscopy is unambiguous, absorption lines should be relatively sharp and assignable to specific hydrogens of the acid base pair just as in gas phase spectra. The lines should be narrower than in the gas phase because there is no rotational structure. Hydrogens in the acid, in the base, nearby, or far from the reactive proton may be selectively activated. 
The system is closed in the sense that the reactive solute is a well defined entity separate from the weakly interacting solvent, in contrast to the water syster. The number of modes present in both the acidic solute and basic solute are known. The number of modes in the non-dye molecule can be varied systematically. .

The concentration of acid-base pairs or of other species added to quench the excitation can be varied and is known. The barrier height can be varied by changing the acidic or basic strength of the non-dye molecule. The system potentially has very rapid time resolution. If two picosecond sources are used, a picosecond resolution, pulse-probe experiment can be performed. The time behavior is obtained simply by measuring total fluorescence as the time delay between the excitation pulse and probe pulse is changed. No expensive streak cameras are needed. 
1. Covington and Dickinson, "Physical Chemistry of Organic Solvent Systems", London.

2. I.M. Kolthoff, Pure and Applied Chemistry 25, 305 (1971).

3. Hayd, Weidermann, and zundel, J. Chem. Phys. 70, 86 (1979).

4. H.P. Fritz and W. Kornrumpf, J. Electroanal. Chem. 100, 217 (1979).

5. Modern Aspects of Electrochemistry, B.E. Conway and J. O'M. Bockris, eds., including V.3, P. 137

6. E. Grunwald and D. Eustace in "Proton Transfer Reactions", V. Gold and E.F. Caldin, eds., Chapman and Hall, London (1975).

7. E.V. Shpol'skii, Sov. Phys. Uspekhi 6, 411 (1963).

8. R.I. Personov and v.v. Solochmov, Opt. Spectrosc. 23, 317 (1967).

9. F.P. Schafer in "Dye Lasers", Topics in Applied Physics, v. 1, F.P. Schafer, ed., Springer-Verlag, New York (1973)

10. B.ti. Robinson in reference 6 .

11. K.H. Drexhage in reference 9.

12. D. Huppert, M. Gutman, and K.J. Kaufmann, Adv. Chem. Phys. 47, 643 (1981).

13. J.E. Crooks in "Comprehensive Chemical Kinetics", v. B, C.H. Bamford and C.F.H. Tipper, eds., Elsevier (1977). 
This report was done with support from the Department of Energy. Any conclusions or opinions expressed in this report represent solely those of the author(s) and not necessarily those of The Regents of the University of Califorsia, the Lawrence Berkeley Laboratory or the Department of Energy.

Reference to a company or product name does not imply approval or recommendation of the product by the University of California or the U.S. Department of Energy to the exclusion of others that may be suitable. 